\title{
Protection, adaptation or cell death as response to toxins
}

Citation for published version (APA):

Sthijns, M. M. J. P. E. (2017). Protection, adaptation or cell death as response to toxins: Dependence of time and dose. [Doctoral Thesis, Maastricht University]. Maastricht University. https://doi.org/10.26481/dis.20170915ms

Document status and date:

Published: 01/01/2017

DOI:

10.26481/dis.20170915ms

Document Version:

Publisher's PDF, also known as Version of record

\section{Please check the document version of this publication:}

- A submitted manuscript is the version of the article upon submission and before peer-review. There can be important differences between the submitted version and the official published version of record.

People interested in the research are advised to contact the author for the final version of the publication, or visit the DOI to the publisher's website.

- The final author version and the galley proof are versions of the publication after peer review.

- The final published version features the final layout of the paper including the volume, issue and page numbers.

Link to publication

\footnotetext{
General rights rights.

- You may freely distribute the URL identifying the publication in the public portal. please follow below link for the End User Agreement:

www.umlib.nl/taverne-license

Take down policy

If you believe that this document breaches copyright please contact us at:

repository@maastrichtuniversity.nl

providing details and we will investigate your claim.
}

Copyright and moral rights for the publications made accessible in the public portal are retained by the authors and/or other copyright owners and it is a condition of accessing publications that users recognise and abide by the legal requirements associated with these

- Users may download and print one copy of any publication from the public portal for the purpose of private study or research.

- You may not further distribute the material or use it for any profit-making activity or commercial gain

If the publication is distributed under the terms of Article $25 \mathrm{fa}$ of the Dutch Copyright Act, indicated by the "Taverne" license above, 


\section{Protection, adaptation or cell death as response to toxins:

\author{
Dependence on time and dose
}

Mireille Sthijns 
Protection, adaptation or cell death as response to toxins:

Dependence on time and dose

Thesis, Maastricht University, Maastricht, The Netherlands

Layout: Mireille Sthijns

Cover design: Dennis Wouters

Printed by: $\quad$ Gildeprint

ISBN: $\quad 978-94-6233-656-8$

(C) Mireille M.J.P.E. Sthijns, 2017 


\title{
Protection, adaptation or cell death as response to toxins: \\ Dependence on time and dose
}

\author{
PROEFSCHRIFT \\ ter verkrijging van de graad van doctor aan de Universiteit Maastricht, \\ op gezag van de Rector Magnificus, Prof. Dr. Rianne M. Letschert \\ volgens het besluit van het College van Decanen, \\ in het openbaar te verdedigen \\ op vrijdag 15 september 2017 om 10.00 uur
} door

Mireille Maria Johanna Petronella Elisabeth Sthijns

geboren te Maastricht op 25 februari 1991 


\section{Promotores}

Prof. Dr. A. Bast

Prof. Dr. G.R.M.M. Haenen

\section{Co-promotor}

Dr. A.R. Weseler

\section{Beoordelingscommissie}

Prof. Dr. O. Bekers (voorzitter);

Dr. G. Nicolaes;

Prof. Dr. H.A.J. Struijker Boudier;

Prof. Dr. R. Morgenstern, Karolinska Institutet, Solna, Sweden;

Prof. Dr. F.G.M. Russel, Radboudumc Nijmegen.

The research described in this thesis was conducted at NUTRIM School of Nutrition and Translational Research in Metabolism of Maastricht University. 


\section{Contents}

$\begin{array}{lll}\text { Chapter } 1 & \text { Introduction and aims of the thesis } & 7\end{array}$

Chapter 2 Time in redox adaptation processes: from evolution to 53 hormesis.

Chapter 3 Adaptation to acrolein through upregulating the protection $\quad 79$ by glutathione in human bronchial epithelial cells: the materialization of the hormesis concept.

Chapter 4 Silver nanoparticles induce hormesis in A549 human epithelial cells.

Chapter 5 Rutin protects against $\mathrm{H}_{2} \mathrm{O}_{2}$-triggered impaired relaxation of placental arterioles and induces Nrf2-mediated adaptation in Human Umbilical Vein Endothelial Cells exposed to oxidative stress.

Chapter 6 Dual effect of acrolein on microsomal glutathione Stransferase 1 activity: effect of concentration and time.

Chapter 7 Redox modulation determines the fate of human melanoma cells exposed to quercetin and oxidative stress: adaptive antioxidant protection or apoptosis, a matter of life or death.

$\begin{array}{lll}\text { Chapter } 8 & \text { Summary and general discussion } & 225\end{array}$

Chapter 9 Nederlandse samenvatting en algemene discussie 239

Valorization addendum 253

Dankwoord 265

List of publications 273

$\begin{array}{ll}\text { List of abstracts } & 275\end{array}$

$\begin{array}{ll}\text { Curriculum vitae } & 279\end{array}$ 

Chapter 1

Introduction and aims of the thesis 


\section{Introduction}

A molecule of a specific compound consists of a unique combination of one or more different atoms connected to each other. In living cells or organisms all compounds can become a toxin. In toxicology, the adverse effects of compounds on living organisms are studied. Whether a compound is toxic depends on the dose, as Paracelsus in 1500 defined: 'Sola dosis facit venenum' (in English: The dose makes the poison), thus making every compound a potential toxin. A compound can be of chemical, physical or biological origin. In contrast to the general perception, the most potent toxins are of biological origin. Next to the dose and nature of the compound, also the time plays an important role in toxicology. The frequency and time of exposure will affect the biological response. Also on effect level, the parameter time is involved. An acute effect is an effect that occurs within hours or days. A subchronic effect is established within weeks or months, while a chronic effect can only be seen after months or years. Depending on the conditions, a specific effect can be beneficial or detrimental. A drug used to treat a specific disease is intended to cause an effect in a patient (e.g. lowering of blood pressure) that is wanted, while in a healthy person this is an adverse effect. The concentration used in the patients is the effective dose in patients. However in patients, a too high dose of a drug will also cause an undesirable health effect. This concentration is the toxic dose. The final effect of the compound is also affected by other substances or nutrition given. Different compounds given simultaneously can work additive, synergistic or antagonistic towards each other. Before a compound produces an effect in an organism, the compound has to reach its site of action in that organism. The processes involved are absorption, metabolism, distribution, accumulation and excretion. Metabolism mainly takes place in the liver. During phase I metabolism compounds are detoxified or drugs activated or deactivated by reduction, oxidation or hydrolysis, while in phase II metabolism compounds are modified by conjugation: methylation, sulphation, acetylation, glucuronidation, glutathione or lysine conjugation. A compound can have specific effects on a certain organ or lead to systemic effects. The effects on the organism can be cellular, biochemical or macromolecular of nature. For example, cells can be changed e.g. enzyme systems can be affected, protein synthesis can be influenced, deoxyribonucleic acid (DNA) can be damaged, other (reactive) compounds can be produced or cells can die. There are many variants of cell death from which apoptosis, programmed cell death and necrosis are the most important forms (1). This exemplifies the dynamic interplay between the compound and the biological organism in the actual response. In the organism many protective mechanisms have evolved. Additionally, an organism is also able to adapt its protection specific for the compound it is exposed to. In this thesis, we focus on the induced redoxrelated effects by acrolein, nanoparticles and flavonoids (Table 1). Acrolein is studied since 
this is one of the model compounds in toxicology. Nanoparticles are of interest due to their physical properties and the resulting reactivity. The flavonoids are studied because of their huge health effects, while the exact mechanism involved is still enigmatic. Cells are equipped with protection systems, including the glutathione (GSH) and thioredoxin (Trx) system to maintain their redox status within narrow limits. When protection systems are not sufficient to deal with toxins that disturb the redox balance, toxicity arises or diseases develop including chronic obstructive pulmonary disease (COPD), cardiovascular disease (CVD) or cancer. However, paradoxically some forms of "redox toxicity" have a health benefit, which will be further elaborated in this thesis.

\subsection{Compounds}

\subsubsection{Acrolein}

Acrolein is an $\alpha, \beta$-unsaturated aldehyde, also named acrylaldehyde, allyl aldehyde or prop-2-enal (Figure 1). This aldehyde is released during incomplete combustion processes. Incomplete combustion can either start endogenously or exogenously (2).

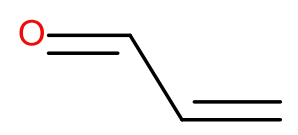

Figure 1: Molecular structure acrolein.

Endogenous sources of acrolein are metabolism of the anti-cancer drug cyclophosphamide, inflammation; acrolein is formed by myeloperoxidase via threonine conversion, and lipid peroxidation where acrolein is formed as a byproduct (2). Acrolein is present in the diesel exhaust of cars, as a byproduct of cooking food or as a waste product of various industrial processes (2). The acrolein released via these processes can be seen as an air pollutant. Another major exogenous source of acrolein is cigarette smoke. Depending on the cigarette brand, each cigarette contains approximately $0.8 \mu \mathrm{mol}$ of acrolein (3). Smoking this cigarette will result in approximately $80 \mu \mathrm{M}$ of acrolein in the respiratory lining fluid (3). The World Health Organization (2002) defined a non-observed adverse effect level (NOAEL) for acrolein of $0.1 \mu \mathrm{g} / \mathrm{m}^{3}$, while the lowest observed adverse effect level (LOAEL) was set at $0.5 \mu \mathrm{g} / \mathrm{m}^{3}$ (0.22 ppm). This means with an ambient concentration of around $0.0087-0.41 \mu \mathrm{g} / \mathrm{m}^{3}$ depending on the environment, the NOAEL is exceeded, whereas the LOAEL is still adhered (4). However, smoking one cigarette could induce a level of $90 \mathrm{ppm}$ of acrolein, which is by far exceeding the LOAEL. Acrolein exposure has induced a decreased respiratory function in 2.5 of 1000 people in 2007, in general in the United States (5). Adverse health effects due to acrolein exposure depend 
on the route of exposure and are respiratory, ocular, and gastrointestinal irritations due to epithelial hyperplasia, ulceration, and hemorrhage (6). The major route of acrolein exposure is via inhalation. Acrolein can also be ingested or exposed to the skin. Acrolein is water soluble and $80 \%$ of the acrolein reacts in the upper respiratory tract, whereas only $20 \%$ reaches the lower parts of the lung including bronchioli and alveoli (7). When acrolein is present in the human body, it is taken up by the cells mainly via passive diffusion (8). In the cell, acrolein reacts with protein or DNA, resulting in acrolein adducts that damage the DNA or affect protein function. Acrolein is a soft electrophile, that reacts mainly with soft nucleophiles, according to the 'Hard and Soft (Lewis) Acids and Bases' (HSAB) principle (9). The physiological most kinetically favorable reaction is the 1,4 Michael addition of acrolein on the thiol present on the $\beta$ carbon of cysteine residues in proteins (10-12). Acrolein can also form an adduct the $\varepsilon$-amine of lysine residues or the imidazole group of histidine residues via Schiff base formation (13). This Schiff base reaction of acrolein could also result in adducts with deoxyguanosine nucleic acid of DNA (14). Finally, protein cross-links can be formed by Schiff base formation of the carbonyl or via Michael addition bound acrolein (15). Due to acrolein adduct formation, mucociliar transport and alveolar-capillary barrier are deteriorated, DNA replication, transcription and repair is affected and apoptosis, inflammation and cytoskeletal changes are induced (4). In addition, alterations in the nuclear factor kappa-light-chain-enhancer of activated $B$ cells (NF-kB), nuclear factor (erythroid-derived 2)-like 2 (Nrf2) and early growth response (EGR) pathways can be seen (4). This finally contributes to an increased risk on developing diseases like asthma or COPD, where an increased level of acrolein is found in expired breath or sputum of exposed individuals (4).

\subsubsection{Flavonoids}

Flavonoids are polyphenolic compounds, mainly present in vegetables and fruit and known because of their antioxidant properties (16). Quercetin and rutin are the two most abundant flavonoids in nature. Quercetin is mostly present in apples and onions and depending on the consumption of food, daily intake is estimated to be approximately 20$35 \mathrm{mg} /$ day $(17,18)$. Second, the intake of rutin, which is mainly present in buckwheat and citrus fruits, is averaged to be around $5-10 \mathrm{mg} /$ day (19). Over 4000 flavonoids exist and their chemical structure is identified as a benzo- $\psi$-pyrone derivative consisting of in total 3 ( $A, B$ and $C$ ) phenolic and pyrane rings (Figure 2A). Various classes can be distinguished according to the different substitutions of hydroxyl, methoxy, and glycosidic side groups or the conjugation between the A- and B- rings. Quercetin belongs to the flavonols (Figure $2 B$ ) and has a hydroxyl group at the 3 position in the $C$ ring, at the 5 and 7 position of the $A$ ring and at the $3^{\prime}$ and $4^{\prime}$ position of the $B$ ring (Figure $2 \mathrm{C}$ ). Rutin is part of the flavone class (Figure 2D) and has a hydroxyl group at the 5 and 7 position of the $A$ ring and at the $3^{\prime}$ and 
$4^{\prime}$ position of the $B$ ring (Figure 2E). Additionally, a rutinose group is present at the 3 position of the $\mathrm{C}$ ring (Figure $2 \mathrm{E}$ ).

A.<smiles>c1ccc(C2CCc3ccccc3O2)cc1</smiles>

B.<smiles>O=c1c(O)c(-c2ccccc2)oc2ccccc12</smiles>

D.<smiles>O=c1cc(-c2ccccc2)oc2ccccc12</smiles>

c.<smiles>O=C1c2c(O)cc(O)cc2OC(c2ccc(O)c(O)c2)C1O</smiles>

E.<smiles>CC1C(O)C(O)C2OCC3OC(C(O)C(O)C3O)C(OC3C(=O)c4c(O)cc(O)cc4OC3c3ccc(O)c(O)c3)C1O2</smiles>

Figure 2: Flavonoid backbone (A); Flavonoids are divided in several classes from which the flavonols (B) and flavones (D) are two classes; quercetin belongs to the flavonols (C), whereas rutin $(E)$ is part of the flavones and has a characteristic rutinose group, a sugar group of glucose and rhamnose coupled, at the 3 position of the $C$ ring (20).

Depending on their difference in structure these flavonoids also have a difference in reactivity. For example the antioxidant effect of quercetin is much higher than the antioxidant function of rutin, having a trolox equivalent antioxidant capacity (TEAC) value of respectively 4.7 and $2.4 \mathrm{mM}(16)$. Due to this antioxidant function, both quercetin and 
rutin scavenge radicals and are thereby converted to their oxidized forms, called quinones (21) (Figure 3).

\section{A. Quercetin quinone}<smiles>O=C1C=CC(c2oc3cc(O)cc(O)c3c(=O)c2O)=CC1=O</smiles><smiles>O=C1C=C(O)c2c(O)c(=O)c(=C3C=CC(=O)C(O)=C3)oc2=C1</smiles><smiles>O=C1C=CC(=c2oc3c(c(O)c2=O)C(=O)C=C(O)C=3)C=C1O</smiles><smiles>O=C1C=CC(=C2Oc3cc(O)cc(O)c3C(=O)C2=O)C=C1O</smiles>

\section{B. Rutin quinone}<smiles>CC1C(O)C(O)C2OC(OCC3OC1C(OC1C(=O)c4c(O)cc(O)cc4OC1C1=CC(=O)C(=O)C=C1)C(O)C3O)C(O)C2O</smiles>

Figure 3: Tautomeric forms of the quinone structures of quercetin (A) and rutin (B). 
Due to its structure quercetin has four tautomeric forms of its oxidation product with the carbonyl group at the $3^{\prime}$ and $4^{\prime}$ position of the $B$ ring and the 4 position of the $C$ ring, at the 7 position of the $A$ ring and the $4^{\prime}$ position of the $B$ ring and at the 3 position of the $C$ ring, at the 5 position of the $A$ ring and the $4^{\prime}$ position of the $B$ ring and the 3 position of the $C$ ring, and at the $4^{\prime}$ position of the $B$ ring and at the 3 and 4 position of the $C$ ring (Figure $3 \mathrm{~A}$ ). The first is an o-quinone, whereas the other three are quinone methides, from which the first one is the most abundant. For rutin only one oxidation product exists with the carbonyl group at position $3^{\prime}$ and $4^{\prime}$ of the $\mathrm{B}$ ring and at position 4 of the $C$ ring (Figure $3 \mathrm{~B}$ ). No other tautomers are possible, because of the presence of the rutinose group. Also the interplay with the endogenous antioxidant system is different with rutin due to its rutinose group being a harder electrophile, while quercetin is a softer electrophile that, according to the HSAB principle, prefers to react with more softer nucleophiles like thiol groups of GSH or proteins, whereas rutin probably reacts more easily with harder nucleophiles like vitamin C (22). The quinone form of both flavonoids however can react with GSH, one better than the other, resulting in glutathionyl adduct formation at position 6 and 8 of the A ring from quercetin and at position 2 ' of the B ring of rutin (21).

When ingested around $38 \%$ of the flavonoids are taken up by the small intestine. In the body, they are distributed and an accumulation of quercetin and its metabolites is mainly seen in plasma, lungs, testes, kidney and heart of rats or pigs fed with a flavonoid rich diet (23). Flavonoids are not only transported in their original form, they are also able to bind to proteins like albumin, increasing its volume of distribution (24). Furthermore, metabolism of flavonoids is performed in the liver, resulting in methylation, glucuronidation and sulphation of the flavonoid (25). Depending on the species and flavonoid, metabolism towards its methylated form or glucuronidated form is more dominant. In rats supplemented with $100 \mathrm{mg} / \mathrm{kg}$ quercetin, after 2 hours $58 \%$ is glucuronidated, $32 \%$ sulphated and $9 \%$ methylated (26). In humans, due to the pharmacokinetics the bioavailability of quercetin is based on a range of studies and the Cmax is approximately $1.46 \pm 0.45 \mu \mathrm{M}$ in plasma at a tmax of $1.1 \pm 0.3$ hours, whereas rutin has a lower $C \max$ of $0.20 \pm 0.06 \mu \mathrm{M}$ at a later timepoint of $6.5 \pm 0.7$ hours (tmax) (27). With supplementation even higher concentrations can be achieved. Rats that received either 75 $\mathrm{mg}$ of quercetin or $150 \mathrm{mg}$ of rutin had approximately $20 \mu \mathrm{M}$ flavonoid in plasma after 1 hour, whereas the peak concentration for quercetin is $90 \mu \mathrm{M}$ and for rutin approximately $55 \mu \mathrm{M}$ in the blood plasma after 10 hours (28). After 24 hours the blood plasma concentration was around 50 and $35 \mu \mathrm{M}$ for quercetin and rutin respectively (29). Supplementation with $100 \mathrm{mg}$ of quercetin for two weeks resulted in blood plasma concentrations of around $0.3 \mu \mathrm{M}$ in humans (18). Furthermore in human volunteers, 
supplemented with $100 \mathrm{mg}$ of rutin, approximately a concentration of $0.2 \mu \mathrm{M}$ could be measured in blood plasma (30). Both quercetin and rutin are taken up by cells, as shown in both Caco-2 and HepG2 cells with radiolabeled $\left[{ }^{14} \mathrm{C}\right]$ quercetin $(31,32)$. The mechanism of uptake of flavonoids is not completely elucidated yet. There are some indications that the SGLT1 transporter is involved and glucose moieties enhance the uptake (33). In the cell, the flavonoid can function as an antioxidant and scavenge radicals (34). During this radical scavenging, the flavonoid gets oxidized and the oxidized product, the quinone, can react with important cellular proteins (35). Previously, it has been seen that the flavonoid forms an adduct on important thiol containing proteins (35). Apparently, this binding can be reversible as indicated for quercetin and GSH and mercaptoethanol (36). Thiol reactivity can be positive when adduct formation enhances endogenous antioxidant systems, but when the function of important cellular proteins is impaired, thiol reactivity of the quinone is considered to be disadvantageous, also defined as the quercetin paradox (35, 37,38 ). The adduct formation of quercetin quinone has also been shown in vivo. In the bile fluid of human volunteers supplemented with quercetin a GSH-quercetin adduct has been detected (39). Finally, quercetin is eliminated much faster with a half-life of $17.9 \pm 2.2$ hours, whereas rutin has a half-life of $19.9 \pm 8.1$ hours (27). Elimination of flavonoids is mainly via the kidneys and the liver by urinary and bile excretion (29).

\subsubsection{Silver nanoparticles}

Silver nanoparticles (AgNPs) are small particles of silver between 1 and $100 \mathrm{~nm}$ in size (40). They differ from bulk silver, because they have a larger surface area, are more reactive, have a higher oxidative capacity, bind biomolecules and are actively taken up (41). On the other hand ionic silver has no surface area at all and can form precipitates and complexes with both inorganic and organic compounds (41). Two different synthesis methods exist: a top-down and a bottom-up approach. An example of the top-down approach is the formation of AgNPs by radiation of bulk silver (40). In contrast the bottomup synthesis is achieved by strong chemical reduction of for example sodium borohydride (40). Depending on the $\mathrm{pH}$, temperature and coating agent, the shape and physicochemical properties of the nanoparticles can be finetuned (40). The applications of AgNPs are in approximately in 600 tons of products in 2016 and expected to double by 2022. The content of silver in these products differs, for example a bandage contains 1133 $\mathrm{mg} / \mathrm{kg}$ of silver compared to pants that have approximately $4 \mathrm{mg} / \mathrm{kg}$ of silver (42). There is an enormous range of areas of applications including textile, food and industrial products like sunscreens, cosmetics, medical applications like bone cement, medical devices and wound dressings, biosensors and electronics. Depending on the physicochemical properties, the characteristics and reactivity of the nanoparticles are/is different. For example, depending on their size different levels of cytotoxicity have been observed (43, 
44). Depending on their different physicochemical properties also different types of AgNPs can be defined ranging from NM300, Ag20, Ag300, Ag23 and Ag27 (44).

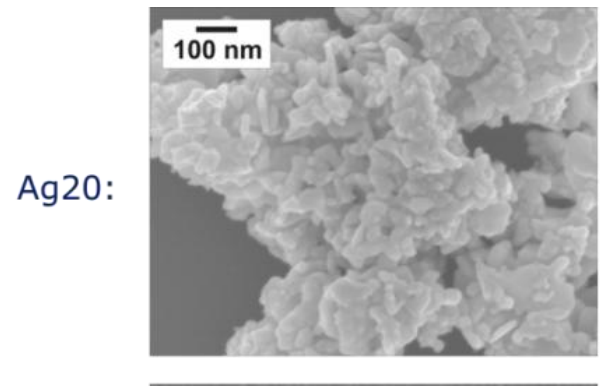

- Ag20:

compact, near-spherical to elongated primary particles which tend to form compact agglomerates/aggregates

- NM300: spherical particles
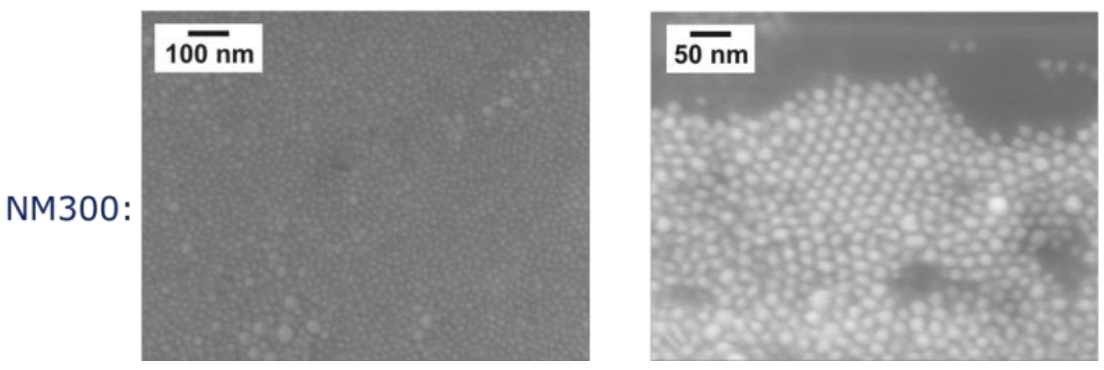

Figure 4: Two different types of silver nanoparticles. Adapted from Sthijns et al. (45).

Next to size also surface charge, aggregation and agglomeration ability can differ (Figure 4). There can be differences in shape and morphology, purity of the particle, chemical composition, surface chemistry and reactivity, solubility, ability to form crystals, concentration, coating like citrate or polyvinylpyrrolidone (PVP) and interaction with the medium (41). The major routes of exposure to AgNPs are inhalation, ingestion or dermal exposure (46). When exposed to AgNPs they are taken up by the body and distributed and accumulated at various locations in the body. Not many studies are performed on the bioavailability of AgNPs after exposure. However, based on some preliminary data from Sprague-Dawley rats a NOAEL of $100 \mu \mathrm{g} / \mathrm{m}^{3}$ or $0.1 \mu \mathrm{g} / \mathrm{l}$ has been defined (47). Oral administration of $1 \mathrm{mg} / \mathrm{kg}$ AgNPs showed a bioavailability of $1.2 \%$, while exposure to 10 $\mathrm{mg} / \mathrm{kg}$ resulted in $4.2 \%$ bioavailability (48). The average daily intake of silver nanoparticles independent of the route of exposure is estimated to be approximately $480 \mu \mathrm{g} / \mathrm{day}(48)$. Bioavailability of AgNPs has not been completely elucidated, some studies show an indication of a tmax of 9 days and a Cmax of $56.8 \mu \mathrm{g} / \mathrm{l}$ in serum after dermal exposure to wound dressings (48). Even long term accumulation could be detected, a medium serum level of about $0.8 \mu \mathrm{g} / \mathrm{l}$ was measured 6 months after the use of these wound dressings (48). In the body, AgNPs could mainly be detected in the kidneys, liver, spleen, brain and lung tissue (49). AgNPs are taken up by cells, their presence has been identified in hepatic 
and renal tubular epithelial cells (49). Additionally, their uptake has been shown to be via endocytosis or active transport in which the high affinity $\mathrm{Cu}$ transporter (CTR1) and divalent metal transporter (DMT1) have been shown to play an important role (40). The toxicity of AgNPs and mechanism behind it is still subject of investigation, but some studies show that an increase in the concentration of AgNPs is associated with an increase in the level of oxidative stress measured $(43,50)$. Finally, excretion of AgNPs occurs predominantly via the urine and feces (51).

Table 1: Overview of properties of different chemicals.

\begin{tabular}{|c|c|c|c|c|}
\hline Compound & Acrolein & Quercetin & Rutin & Silver nanoparticles \\
\hline $\begin{array}{l}\text { Chemical } \\
\text { nature }\end{array}$ & Gas & Solid & Solid & Nanoparticulate \\
\hline Main source & $\begin{array}{l}\text { Cigarette } \\
\text { smoke }\end{array}$ & $\begin{array}{l}\text { Apples and } \\
\text { onions }\end{array}$ & $\begin{array}{l}\text { Buckwheat and } \\
\text { citrus fruits }\end{array}$ & $\begin{array}{l}\text { All kind of } \\
\text { applications ranging } \\
\text { from bone cement, } \\
\text { wound dressings to } \\
\text { electronics and cloths }\end{array}$ \\
\hline NOAEL & $0.1 \mu \mathrm{g} / \mathrm{m}^{3}$ & - & - & $100 \mu \mathrm{g} / \mathrm{m}^{3}$ or $0.1 \mu \mathrm{g} / \mathrm{l}$ \\
\hline $\begin{array}{l}\text { Main route of } \\
\text { exposure }\end{array}$ & Inhalation & Ingestion & Ingestion & $\begin{array}{l}\text { Inhalation, Ingestion, } \\
\text { Dermal exposure }\end{array}$ \\
\hline $\begin{array}{l}\text { Cellular } \\
\text { uptake }\end{array}$ & $\begin{array}{l}\text { Passive } \\
\text { diffusion }\end{array}$ & Transporters & Transporters & Endocytosis \\
\hline Metabolism & $\begin{array}{l}\text { GSH } \\
\text { conjugation }\end{array}$ & $\begin{array}{l}\text { Glucuronidation } \\
\text { methylation and } \\
\text { sulphation }\end{array}$ & $\begin{array}{l}\text { Glucuronidation } \\
\text { methylation and } \\
\text { sulphation }\end{array}$ & Not known \\
\hline Bioavailability & $\begin{array}{l}80 \% \text { upper } \\
\text { respiratory } \\
\text { tract }\end{array}$ & $\begin{array}{l}38 \% \text { intestinal } \\
\text { uptake, } \mathrm{Cmax}= \\
1.46 \pm 0.45 \mu \mathrm{M} \text { in } \\
\text { plasma, tmax = } \\
1.1 \pm 0.3 \text { hours, } \\
\mathrm{t}_{1 / 2}=17.9 \pm 2.2 \\
\text { hours }\end{array}$ & $\begin{array}{l}38 \% \text { intestinal } \\
\text { uptake, } \mathrm{Cmax}= \\
0.20 \pm 0.06 \mu \mathrm{M} \text { in } \\
\text { plasma, tmax = } \\
6.5 \pm 0.7 \text { hours, } \\
\mathrm{t}_{1 / 2}=19.9 \pm 8.1 \\
\text { hours }\end{array}$ & $\begin{array}{l}1.2-4.2 \% \text { via } \\
\text { ingestion } \\
\text { Cmax }=56.8 \mu \mathrm{g} / \mathrm{l} \text { in } \\
\text { plasma } \\
\text { tmax }=9 \text { days }\end{array}$ \\
\hline Excretion & $\begin{array}{l}\text { Urine and } \\
\text { bile }\end{array}$ & Urine and bile & Urine and bile & Urine and bile \\
\hline
\end{tabular}




\subsection{Protection}

\subsubsection{Oxidative stress}

An electrophile is defined as an electron acceptor, whereas a nucleophile is defined as an electron donor. A pro-oxidant is a compound that generates reactive oxygen species (ROS) or inhibits antioxidants, thereby contributing to the formation of oxidative stress. An antioxidant is a molecule that counteracts the oxidation of substances (52).

When the protective antioxidant mechanisms are overwhelmed by the production of oxidants, this is defined as oxidative stress. Different types of reactive species exist that function as oxidant. Physiologically especially the partly reduced forms of oxygen and nitrogen are important, which are called ROS and reactive nitrogen species (RNS) (Table 2). These reactive species differ in their reactivity for example the radicals, in particular hydroxyl radical $\mathrm{OH}^{-}$and the non-radical $\mathrm{ONOO}^{-}$belong to the most reactive species (53). From hydrogen peroxide $\left(\mathrm{H}_{2} \mathrm{O}_{2}\right)$, for example the concentration is defined to be within the $\mathrm{nM}$-low $\mu \mathrm{M}$ range, it has been shown to react within $1 \mathrm{~ms}(54,55)$. The other ROS, especially the radicals react even faster. RNS on the other hand are physiologically present in a concentration range of $100 \mathrm{pM}$ to $5 \mathrm{nM}$, however their reaction rate is much slower, reacting within 0.1 to 2 seconds (56). Additionally, also the location of reactivity varies between the reactive species. Some are able to be transported over membranes and induce their reactivity on sites relative far away from the site of production. These species are thereby capable of inducing a more spread reactivity and could induce a signaling pathway or toxicity at a larger distance.

Table 2: Types of reactive species, figure adapted from Caimi et al. 2004 and Adly et al. 2010 (57).

\section{Types of Reactive oxygen species (ROS)}

\section{Radicals}

\begin{tabular}{|l|l|l|l|}
\hline Superoxide & $\mathrm{O}_{2} \cdot$ & Hydrogen peroxide & $\mathrm{H}_{2} \mathrm{O}_{2}$ \\
Hydroxyl & $\mathrm{OH} \cdot$ & Hypochlorous acid & $\mathrm{HOCl}$ \\
Peroxyl & $\mathrm{RO}_{2} \cdot$ & Hypobromous acid & $\mathrm{HOBr}$ \\
Alkoxyl & $\mathrm{RO}^{-}$ & Ozone & $\mathrm{O}_{3}$ \\
\hline
\end{tabular}




Types of Reactive nitrogen species (RNS)
Radicals
\begin{tabular}{|l|l|l|l|} 
Nitric oxide & $\mathrm{NO} \cdot$ & Non-radicals & $\mathrm{NO}_{2}$ \\
Nitrous acid & $\mathrm{NHO}_{2} \cdot$ & Nitrosyl cation & $\mathrm{NO}^{+}$ \\
& & Nitrosyl anion & $\mathrm{NO}^{-}$ \\
& & Dinitrogen tetraoxide & $\mathrm{N}_{2} \mathrm{O}_{4}$ \\
& & Dinitrogen trioxide & $\mathrm{N}_{2} \mathrm{O}_{3}$ \\
& & Peroxynitrite & $\mathrm{ONOO}$ \\
& & Peroxynitrous acid & $\mathrm{ONOOH}$ \\
\hline & & Alkylperoxynitrites & $\mathrm{ROONO}$ \\
\hline
\end{tabular}

Oxidants are produced both endogenously as well as exogenously. An endogenous source of oxidants is metabolism. For example during energy production, the oxidative phosphorylation in mitochondria or beta oxidation of fatty acids by peroxisomes oxidants are generated (Figure 5). Furthermore, also other metabolic enzymes can form reactive species, like xanthine oxidase, lipoxygenase, cyclooxygenase, cytochrome P-450 monooxygenase. In addition, also during inflammation oxidants can be formed. For example, the membranes of neutrophils contain reduced nicotinamide adenine dinucleotide phosphate (NADPH) oxidases that can form reactive species that are involved in the defense against pathogens. Next to NAPDH oxidases, also other enzymes are involved in inflammation and generate reactive species, e.g. the granulocytic enzymes eosinophil peroxidase and myeloperoxidase (MPO). Exogenous sources of oxidants can be pollution, metals (via the Fenton and Haberweiss reaction different radicals are formed), smoking, radiation, products produced by bacteria or viruses, medication, cold, excessive exercise or intake of sugar or fat (58).

Reactive species can be involved in various signaling pathways. They regulate processes like proliferation, metabolism, differentiation and survival by targeting apoptosis signal-regulated kinase 1 (ASK1), phosphoinositide 3 kinase (PI3K), protein tyrosine phosphatase (PTP), and src homology 2 domain-containing (Shc) (60). In addition, they influence the endogenous antioxidant and anti-inflammatory response by affecting Trx, redox factor-1 (Ref-1) or Nrf2 (60). Furthermore, targeting iron regulatory protein (IRP) or ataxia-telangiectasia mutated (ATM), results in effects within iron homeostasis and the DNA damage response (60). Specificity, selectivity and compartmentalization of the reactive species are essential for effective redox signaling. Various hypotheses are developed how these reactive species induce redox signaling. The first is the thermodynamic concept, based on kinetic interactions, most abundant molecules have an increased chance to interact (61). 
A.

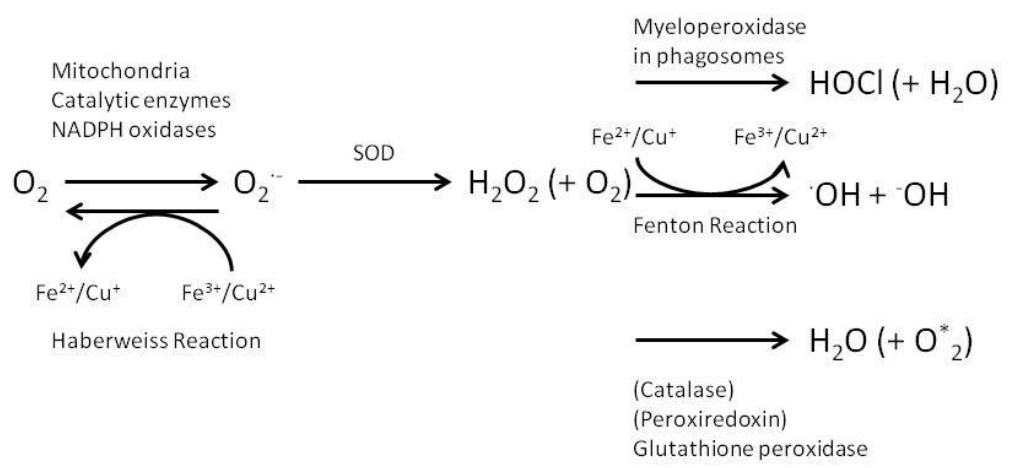

B.

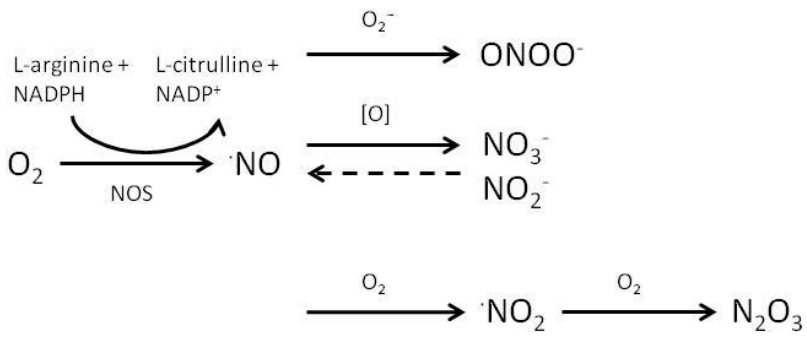

Figure 5: Reactions involved in the formation of reactive oxygen species (ROS) (A) and reactive nitrogen species (RNS) (B), figure adapted from Paulson et al. (59). SOD = superoxide dismutase; $\mathrm{NADPH}=$ reduced nicotinamide adenine dinucleotide phosphate.

The second is the floodgate hypothesis, a specific radical interacts with a target, that induces a subsequent pathway (61). The last one, is about the preference of ROS for certain sensor proteins (61). In redox signaling oxidation of thiols present in cysteine residues of important proteins is important. Depending on the reactive species, pKa of the thiol (in comparison pKa cysteine 8.3 versus pKa selenocysteine 5.47 ), accessibility of the thiol within the 3D structure and amino acid sequence of the protein (more hydrophilic or hydrophobic, steric hindrance), environmental characteristics $(\mathrm{pH}$, temperature), abundance of the protein and presence of other molecules or proteins, the specificity and selectivity of reactive species with a specific thiol are defined $(62,63)$. When a thiol is deprotonated, at a physiological $\mathrm{pH}$ a thiolate anion $\left(\mathrm{S}^{-}\right)$is formed that can be target of oxidation itself (64). This will lead to the formation of a sulfenic acid ( $\mathrm{SOH})$. The sulfenic form can further be oxidized to sulfinic $\left(\mathrm{SO}_{2} \mathrm{H}\right)$ or sulfonic $\left(\mathrm{SO}_{3} \mathrm{H}\right)$ species (64). Remarkably, 
the last two mentioned species cannot be reduced by endogenous antioxidant defense mechanisms and induce an irreversible change in protein structure, which is often correlated with an irreversible change in its function.

Next to oxidative changes in protein structures, also cellular lipids and DNA can be affected by reactive species. If this damage is persistent, and cannot be repaired, it will contribute to many pathological conditions (Table 3).

Table 3: Oxidative stress-related diseases, adapted from Rahal et al. (65).

\begin{tabular}{|l|l|l|}
\hline Etiology & Disease & Organ involved \\
\hline $\begin{array}{l}\text { Reactive oxygen intermediates (ROI) } \\
\text { Superoxide dismutase, catalase, } \\
\text { glutathione reductase, glutathione } \\
\text { peroxidase }\end{array}$ & Dacular degeneration & Eyes \\
C-reactive protein & & Multiorgan \\
Reduced NADPH oxidase system & Chronic fatigue & \\
$\begin{array}{l}\mathrm{R}_{0} \text { ribonucleoprotein } \\
\text { Reactive oxygen species (ROS) }\end{array}$ & Atherosclerosis & Multiorgan \\
ROS particularly $\mathrm{H}_{2} \mathrm{O}_{2}$ & Autoimmune disorders & Blood vessels \\
Radical oxygen species & Neurodegenerative diseases & Brain \\
& Asthma & Lungs \\
Glutathione transferase kappa (GSTK 1-1) & Rephritis & Joints \\
Pathophysiological processes including & Melanoma & Kidney \\
DNA damage and lipid peroxidation (LPO) & & Skin \\
Reactive oxygen species (ROS) & Myocardial infarction & Heart \\
\hline
\end{tabular}

Various biomarkers are used to determine whether there is oxidative stress and to what extent (66). Oxidative damage to DNA is measured by measuring oxidized bases (marker of exposure) or strand breaks (functional marker). On the other hand, lipid damage is mostly examined by measuring breakdown products of lipid peroxidation including malondialdehyde (MDA) or dienes via different methods (marker of exposure) (66). Additionally, protein oxidation is measured by determination of carbonyls, thiols, nitrated amino acid, protein Tyr oxidation products, (bityrosine) protein Trp oxidation products, methionine sulphoxide, hydroxides/hydroperoxides, protein 2-adipic semi aldehyde, neoepitopes (66). Next to this also the balance between oxidants and antioxidants could be determined as well as the pathological index of oxidative stressrelated diseases. The total antioxidant capacity can be determined by TEAC assay, the ferric reducing antioxidant power (FRAP) assay, the oxygen radical absorbance capacity 
(ORAC) assay or the LDL lag time (66). In addition, the GSH/glutathione disulfide (GSSG) ratio can be shown as well as the ability to resist strand breaks (66).

To protect against oxidative stress, different intrinsic endogenous protection mechanisms exist $(65,67)$. First, cytochrome P450 enzymes are involved in the protection against oxidative stress. Second, different antioxidant enzymes contribute to the defense against oxidative stress including superoxide dismutase, catalase and different enzymes of the glutathione and thioredoxin system. Third, different efflux transporters can offer protection. Finally, vitamins ( $C, E$ and $A$ ) form an important part of the endogenous antioxidant network. Some are essential and can only be obtained via nutrition, whereas others can also be produced in the body itself. In this thesis, the main focus is on two central endogenous antioxidants systems with their major participants and interplay: the glutathione and thioredoxin system.

\subsubsection{The glutathione system}

Glutathione is a tripeptide consisting of three amino acids: L-glutamic acid, Lcysteine, and glycine $(68,69)$. Glutathione exists in a reduced and an oxidized form: reduced glutathione (GSH) and glutathione disulfide (GSSG) respectively. The GSH synthesis proceeds in two steps (Figure 6).

The GSH synthesis can be regulated by the concentration of GSH present in the cell. In cells, the GSH levels vary between 0.5-10 mM $(68,69)$. However, in most cells the GSH concentration is between 1-2 mM, whereas in hepatocytes it can reach levels around $10 \mathrm{mM}$. Additionally, not all of the GSH is present in the reduced form. Depending on the redox status of the cell, the ratio between GSH and GSSG can vary. Also between different cellular compartments different ratio's of [GSH]/[GSSG] can be found. GSH is present in the cytosol, nucleus and mitochondria in a ratio of [GSH]/[GSSG] of 10:1, whereas in the endoplasmic reticulum the [GSH]/[GSSG] is estimated to be $3: 1(68,69)$. When the GSH level decreases, the GSH synthesis is increased, because of an increase in activity of glutamate cysteine ligase (GCL). GCL has two subunits: a heavy catalytic subunit of $73 \mathrm{kDa}$ and a light regulatory subunit of $28 \mathrm{kDa}$. The catalytic subunit has binding sites for the three substrates, whereas the regulatory subunit has a binding site for GSH. When reduced GSH is present, the regulatory subunit keeps the catalytic subunit inactive, while a low GSH level leads to a disulfide bond between both subunits. This is an effect due to the level of oxidative stress and will result in a conformational change of the catalytic subunit and an increase in activity of the enzyme $(68,69)$. Additionally, a low level of GSH also results in overwhelming ROS that can bind with kelch-like $\mathrm{ECH}$-associated protein 1 (Keap1), the inhibitory protein of Nrf2 and induce its proteasomal degradation. Newly 
synthesized Nrf2 is not inhibited anymore and can translocate to the nucleus, where it results in the expression of endogenous antioxidant enzymes including $\mathrm{GCL}$. This will result both in an increased synthesis of GSH.

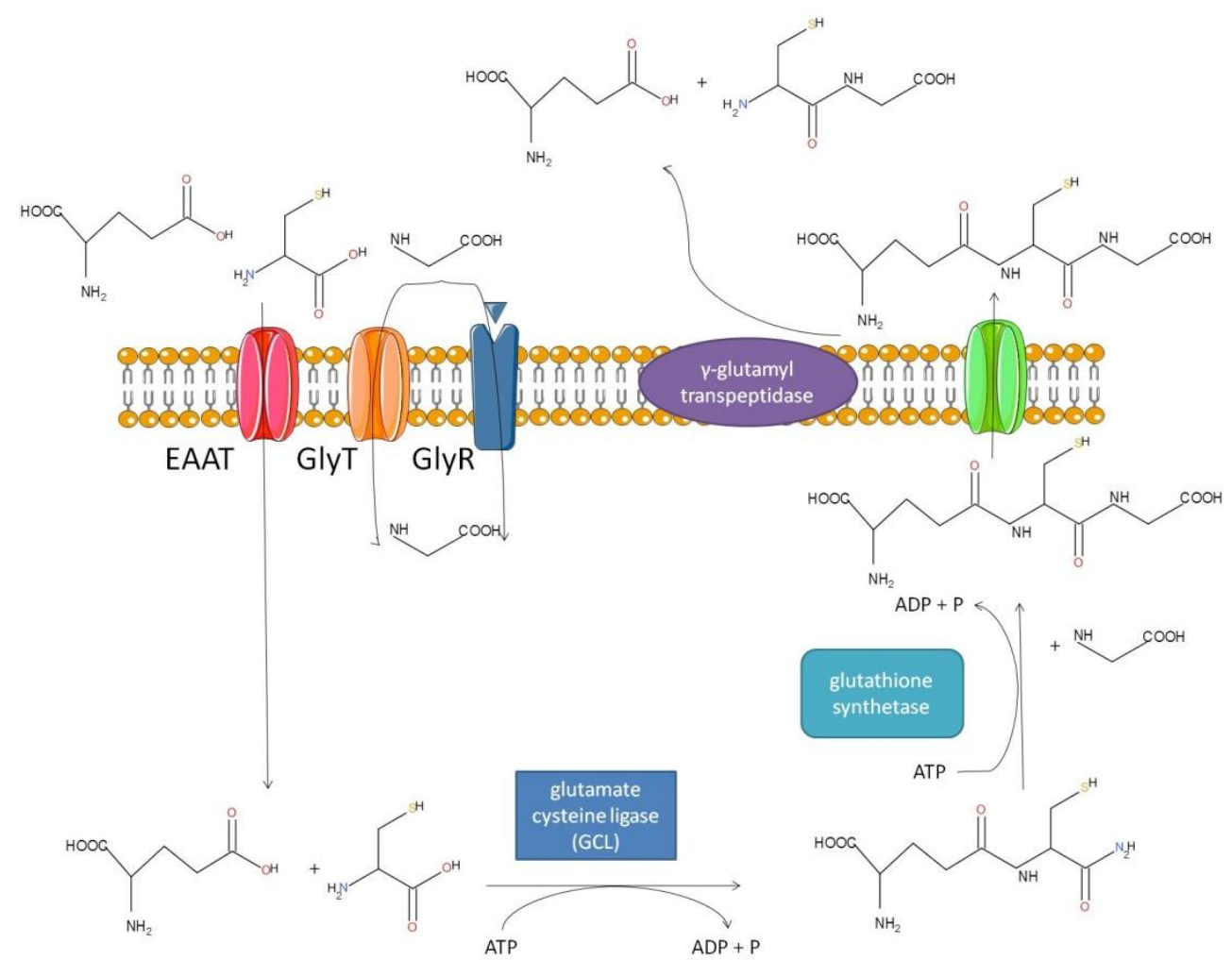

Figure 6: GSH synthesis. The separate amino acids are taken up by specific channels: EAAT, Excitatory amino acid transporters cysteine and GlyR or GlyT (glycine receptor or transporter). Glutamate cysteine ligase (GCL) catalyzes the rate-limiting step of GSH synthesis, hydrolyzing glutamic acid and cysteine by using adenosine triphosphate (ATP). Glutathione synthetase subsequently synthesizes glutathione (GSH) by adding the last amino acid glycine to the tripeptide by using ATP. GSH is transported out the cell by specific transporters. Outside the cell, v-glutamyl peptidase converts GSH to glutamic acid and a peptide of cysteine and glycine. Adapted from Rahman et al. (70)

Various compounds are known to regulate the synthesis of GSH. One of them is the specific inhibitor of the catalytic subunit of GCL: buthionine sulfoximine (BSO) (71). Another is the GSH precursor N-acetylcysteine (NAC). GSH itself cannot be supplemented, since GSH is not taken up as a whole. Either the amino acids are taken up or $\gamma$-glutamyl transpeptidase $(\nu-G T)$, present in the plasma membrane of cells breaks down extracellular 22 
GSH by the y-glutamyl bond (70). Subsequently a dipeptidase is used to hydrolyze cysteinylglycine to the individual amino acids that can be taken up.

GSH is next to its intracellular presence, also present in the extracellular space. For example, in the epithelial lining fluid of the lungs (70). One of the main functions of GSH is that it is part of the antioxidant defense. It maintains the function of important cellular thiol containing proteins and interacts with other (non-enzymatic) antioxidants. Furthermore, it has an important role in the elimination of xenobiotics. It is involved in signal transduction, mainly via glutathionylation (72). Additional roles of GSH are in the metabolism of signaling molecules or hormones like estrogens, leukotrienes and prostaglandins, the formation of deoxyribonucleotides from ribonucleotides and establishment of iron-sulfur clusters in proteins $(68,69)$. During the first process, GSH is oxidized to GSSG. GSH is regenerated by either increasing the GSH synthesis or by glutathione reductase. GSH synthesis is increased by activating GCL and enhancing the transcription of $\mathrm{GCL}$ messenger riobonucleic acid (mRNA). Glutathione reductase uses NADPH as a cofactor.

$\mathrm{GSSG}+\mathrm{NADPH}+\mathrm{H}^{+} \rightarrow 2 \mathrm{GSH}+\mathrm{NADP}^{+}$

GSH is not acting alone by protecting the organism against certain oxidative or xenobiotic stressors. A couple of enzymes are involved in establishing the functions of GSH. One of them are the glutathione transferases (GSTs) $(68,69,73)$. They catalyze the conjugation reaction of GSH with xenobiotics, electrophiles and oxidants (Figure 7). GSTs are present in the cytosol, mitochondria and the membrane of microsomes. Seven classes of human cytosolic GSTs exists $(\alpha, \mu, \pi, \sigma, \phi, \omega, \zeta)$, whereas three membrane bound GSTs exist: microsomal glutathione transferase 1, 2 and 3 . They belong to the membrane associated proteins in eicosanoid and glutathione metabolism (MAPEG) family.
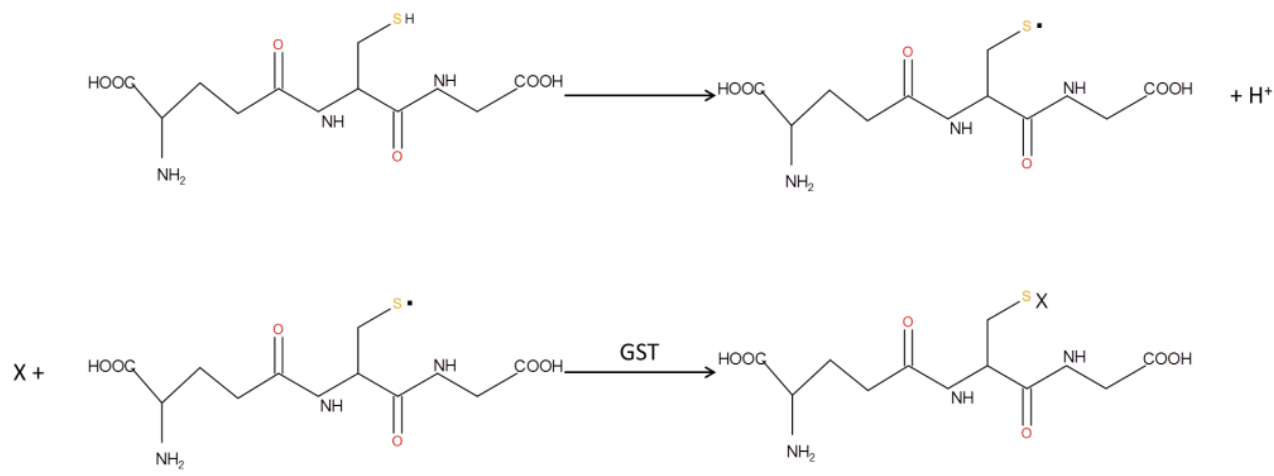

Figure 7: Reaction catalyzed by glutathione transferase (GST). 
Next to this, also glutathione peroxidases (GPxs) exist that either reduce $\mathrm{H}_{2} \mathrm{O}_{2}$ to water or lipid hydroperoxides to their corresponding alcohols $(68,69,74)$. Eight different isoforms are present from which GPx5, 7 and 8 contain a cysteine, whereas the others have a selenocysteine. They differ in expression between different tissues and subcellular localizations. GPX1 is present in the cytoplasm of nearly all different tissues, whereas GPx2 is primarily present in the gastrointestinal tract, GPx3 in plasma and GPx6 is an olfactory enzyme. Additionally, GPx4 preferably catalyzes the reaction of lipid hydroperoxides to either cholesterol or phospholipids. Finally, from GPx7 and 8 an important role in redox signaling has been identified.

$2 \mathrm{GSH}+\mathrm{H}_{2} \mathrm{O}_{2} \rightarrow \mathrm{GS}-\mathrm{SG}+2 \mathrm{H}_{2} \mathrm{O}$

Finally, also glutaredoxins (Grxs) can perform deglutathionylation of proteins or reduce disulfides $(68,69,75)$. Grxs can be regenerated by glutathione reductases (GRs). Thereby GSH, can function in redox signaling, that can finally influence processes like apoptosis, proliferation and differentiation. Thereby GSH can protect or contribute to the development of certain diseases like cancer or COPD.

\subsubsection{The thioredoxin system}

Besides the glutathione system as an endogenous antioxidant system, a second system is active, namely the thioredoxin system. The thioredoxin system contains various players, the main players are oxidized thioredoxin, $\operatorname{Trx}-\mathrm{S}_{2}$, reduced thioredoxin, $\operatorname{Tr} \mathrm{r}-(\mathrm{SH})_{2}$, thioredoxin reductase (TrxR) and NADPH (Figure 8). The cellular concentration of thioredoxin is varying between cell types, for example TrxR is much higher expressed in monocytes, whereas Trx has a high expression in B cells (76). However, compared to GSH, the cellular amount appears to be much lower varying around $0.35 \mathrm{mM}$ instead of 1-10 $\mathrm{mM}$ for GSH (77). However, especially regarding the redox reactivity, especially for thioredoxin reductase the selenocysteine has a lower pKa than cysteine, (5.47 compared to 8.3), probably enhancing its reactivity towards certain substrates in specific environments (78). Thioredoxin has two isoforms, a cytosolic (Trx1) and a mitochondrial one (Trx2). Trx has a conserved region -CGPC- in its active site that plays a major role in its oxidoreductase function. In reduced form it can reduce protein disulfides to their original dithiols, whereby Trx gets oxidized. The main difference between Trx1 and Trx2 is that Trx1 contains two additional thiols that can be posttranslationally modified and thereby regulate the activity of the Trx1 enzyme $(79,80)$. Thioredoxin reductase on the other hand contains three isoforms, TrxR1, TrxR2 and thioredoxin and glutathione reductase (TGR). These isoforms have small structural differences, in addition their subcellular and tissue localization varies. TrxR1 is mainly localized in the cytosol, whereas TrxR2 is expressed in the mitochondria, while the testes are the main location of TGR. Consecutively, also three 
genes are involved in the transcription of the three different isoforms, namely, TXNRD1, TXNRD2 and TXNRD3. TrXR is a dimeric enzyme that contains an N-terminal FAD domain and disulfide motif (C59/C64) and a selenocysteine containing C-terminal -XCUX- motif (81). These domains are involved in its pyridine nucleotide disulfide oxidoreductase activity, whereby oxidized Trx is recycled back to reduced Trx and NADPH is used as a cofactor. Electrons are transferred to the $\mathrm{N}$-terminal disulfide via the flavin adenine dinucleotide (FAD) domain. Subsequently, the electrons are forwarded to the selenothiol motif on the C-terminal, resulting in the reduction of most substrates including Trx and 5,5'-dithiobis-(2-nitrobenzoic acid) (DTNB). However, not all substrates require the last step and presence of the selenothiol. Via this mechanism, the thioredoxin system plays a major role in DNA synthesis, disulfide reduction, apoptosis, transcription factor regulation and denitrosylation $(79,80)$. Depending on its subcellular localization and interaction with other molecules, thioredoxin performs many different functions. In the cytosol it can activate ribonucleotide reductase (RNR) and enhance DNA synthesis and repair. Additionally, it plays a major role in the endogenous antioxidant defense, interacts with many different endogenous antioxidants and is involved in redox signaling. In the nucleus it regulates the activity of many transcription factors, including the redox regulated transcription factors hypoxia-inducible factor (HIF), NF-KB, Nrf2, and p53. Furthermore, it inhibits apoptosis-activator $A S K 1$, thereby regulating proliferation and apoptotic pathways $(79,80)$. Finally, in the plasma membrane it is involved in immunomodulation interacting with Trx1-interacting protein (TXNIP) or extracellularly secreted in interaction with Trx80 it influences chemokine function. Finally, TrxR1 can also be inhibited completely and partially, thereby inducing a pro-oxidant redox cycling function. In case of the last inhibitors, mostly the selenocysteine is targeted (82).

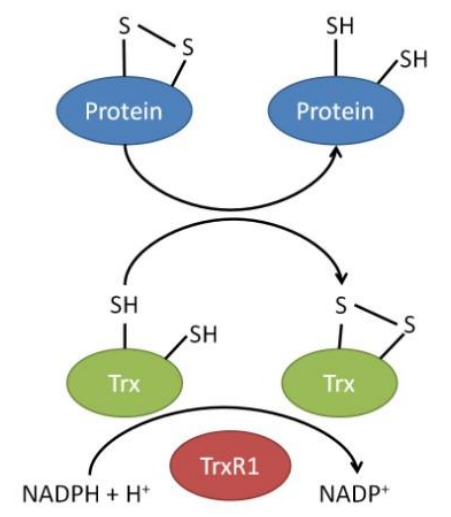

Figure 8: Reaction catalyzed by thioredoxin reductase 1 (TrxR1). Trx = thioredoxin. 


\subsection{Adaptation}

\subsubsection{Redox regulated transcription factors}

Each cell contains different endogenous antioxidant systems including the GSH and Trx system that protect the cell against damage of ROS and electrophiles. However, apart from affecting endogenous antioxidant systems ROS can also induce various other processes. Part of this redox signaling is the regulation of redox-related transcription factors. Different mechanisms have been suggested that result in a change in transcription factor activity and downstream effects. First of all, transcription factors themselves can act as a sensor for, thiol peroxidases, protein phosphatases or kinases, seleno-cysteinecontaining proteins or cytosolic inhibitory complexes. Secondly, a change in the redox status of the cell can result in a change in transcription factor activity and downstream effect. In this thesis, especially mammalian redox regulated transcription factors, with a biological relevance and redox regulated compound will be discussed.

\subsubsection{Nrf2}

The first discussed redox regulated transcription factor is Nrf2. Nrf2 is a protein of $66 \mathrm{kDa}$ consisting of 605 amino acid residues. It is known as a basic leucine Zipper (bZip) transcription factor (83-96). Nrf2 is during basal conditions present in the cytoplasm were it forms a complex with many different co-activators or -inhibitors. One of them is Keap1 that functions as an inhibitor of Nrf2. Next to Keap1 also other inhibitors of Nrf2 have been suggested, including TrxR1 (79). Human Keap1 contains 27 cysteine residues. Keap1 is a cytosolic inhibitory protein that functions due to its high number of thiols as a sensor for oxidative stress and electrophiles. Electrophiles covalently bind Keap1 via S-alkylation. Dependent on the electrophile, a unique set of thiols in Keap1 is adducted. Keap1 is a dimeric protein with two subunits of $69 \mathrm{kDa}$ that consists of 627 amino acid residues. During basal conditions Keap1 forms a complex together with complex of RING-box protein 1 (RBX1) and cullin 3 (cul3) in the cytoplasm. Thereby Keap1 enhances 26Sproteosomal degradation of Nrf2, by facilitating binding of Nrf2 in complex with E3 ligase cul3, which also enhances binding to cytoskeleton structures in the cell, including actin. Keap1 consists of five domains: The N-terminal region (NTR), the Broad complex, Tramtrack, and Bric-a-Brac (BTB) domain, the linker intervening region (IVR), the Kelch domain (or double glycine repeat (DGR) domain) and the C-terminal region (CTR). The BTB domain functions in homodimerization of Keap1, while the IVR contains a nuclear export signal (NES) sequence. Finally, the Kelch domain with six kelch repeats interacts with the Neh2 domain of Nrf2. Nrf2 has 6 Nrf2-ECH homology domains: Neh1, Neh2, Neh3, Neh4, Neh5 and Neh6 domains (97-101). For the interaction with Keap1 the N-terminal Neh2 domain is essential. The central lysine-rich $\alpha$-helix of the Neh2 domain is located in that 
way that it enhances ubiquitination. The Neh4 and Neh5 domain combined form the CREB binding protein (CBP) binding region and the Neh3 domain is named the chromoATPase/helicase DNA binding protein family member 6 (CHD6) binding region. Both regions bind histone acetyltransferases and transactivate other transcription factors. The Neh1 interacts with the DNA and cofactor Maf, while the Keap1-independent degradation of Nrf2 is mediated by the Neh6 domain. Three nuclear localization signals (NLS), NLS1, NLS2 and NLS3, and two nuclear export sequences (NES) NES1 and NES2 are present in Nrf2. During basal conditions, the export signaling overwhelms the import force. However, if Keap1 does not enhance Nrf2 degradation, newly synthesized Nrf2 is not inhibited anymore and can translocate to the nucleus (Figure 9). In addition, electrophile adduction or ROS reactivity with cys183 present in the NES1 could enhance nuclear translocation of Nrf2. In the nucleus, Nrf2 can form a heterodimer with Maf and bind to the electrophile/antioxidant response elements (EpRE/ARE) with consensus sequence TGAG/CNNNGC present in the promoter of several genes encoding endogenous antioxidant genes including heme-oxygenase-1 (HO-1), GCL, TXNRD1 and GST.
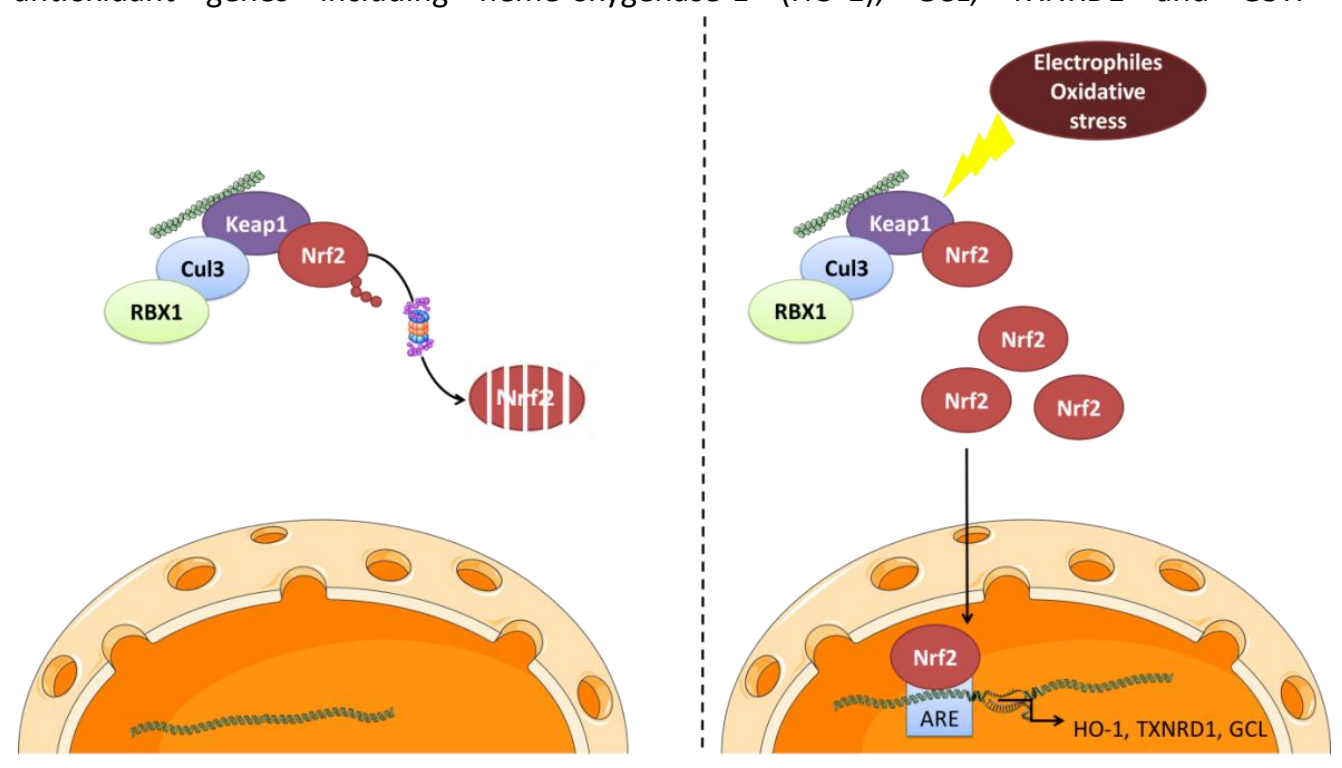

Figure 9: Nrf2 pathway. Keap1 = kelch-like ECH-associated protein 1; Nrf2 = nuclear factor (erythroid-derived 2)-like 2; Cul3 = cullin 3; RBX1 = RING-box protein 1; HO-1 = heme oxygenase 1; TXNRD1 = gene encoding thioredoxin reductase 1; GCL = glutamate cysteine ligase; ARE = antioxidant response element.

The interaction between KEAP1 and Nrf2 has further been investigated. This resulted in the "hinge-and-latch" theory, indicating that Keap1 has a strong binding and weak binding of Nrf2. The strong binding is via the ETGE motif to the Kelch domain of 
Keap1, whereas in the weak binding the DLG motif is involved binding with another kelch domain of Nrf2. The oxidation of Keap1 interferes with both bonds and results in nuclear translocation of newly synthesized Nrf2, while if only the weak binding is deteriorated, Nrf2 is not ubiquitinated and degraded anymore, but it cannot translocate to the nucleus yet (102).

\subsubsection{NF-KB}

NF-KB mainly regulates inflammation and immune responses. There are three different ways to activate NF-KB: the atypical pathway, the canonical-typical or classical pathway and the alternative pathway (103). In addition, there are also different external factors that can result in activation of NF-KB: growth factors, cytokines or bacterial products like lipopolysaccharide (LPS), which is called a pathogen-associated molecular pattern (PAMP). In this introduction, we will mainly focus on the classical pathway activated by PAMPs or cytokines, because that part is known to be redox regulated (Figure 10). PAMPs bind to their toll-like receptor (TLR) and cytokines to their specific receptor, for example tumor necrosis factor $\alpha$ (TNF $\alpha$ ) to the TNFreceptor (TNFR). This activates TGF $\beta$-activated kinase (TAK1) which then phosphorylates and activates IKB kinase $\beta$ (IKK $\beta$ ). IKK $\beta$ is part of the IKK complex. IKK $\beta$ in his turn then phosphorylates and targets proteasomal degradation of the inhibitor of NF-KB, called ІКB. ІкB does not inhibit NF-KB anymore. NF-KB (consisting of $\mathrm{p} 65 / \mathrm{p} 50$ ) is phosphorylated by protein kinase $A$ (PKA) and translocates to the nucleus. In the nucleus coactivators CBP and p300 are recruited and together they bind to NF-KB responsive element (NRE) in the promoters of target genes including interleukin-6 (IL-6), interleukin-8 (IL-8) and TNF $\alpha$. However, in short the atypical pathway is mainly activated by growth factors that initiate the pathway by activating receptor tyrosine kinases, phosphoinositide-3-kinase (Pi3K) and spleen tyrosine kinase (SYK) that subsequently affects downstream IKB and a similar pathway is induced as seen for the classical pathway. Regarding the alternative pathway, NF-KB inducing kinase-1 (NIK1), then IKB kinase $\alpha(I K K \alpha)$ is activated that induces p100 release from RelB that subsequently interacts with $\mathrm{p} 52$, which enters the nucleus and influences proinflammatory gene expression. NF-KB is the first transcription factor from which it is discovered that it is redox regulated. At many points in this pathway oxidation and reduction can result in a different regulation of NF-KB mediated genes. One example is cys62 of p50 which can be oxidized that diminishes DNA binding of the corresponding transcription factor (104). 


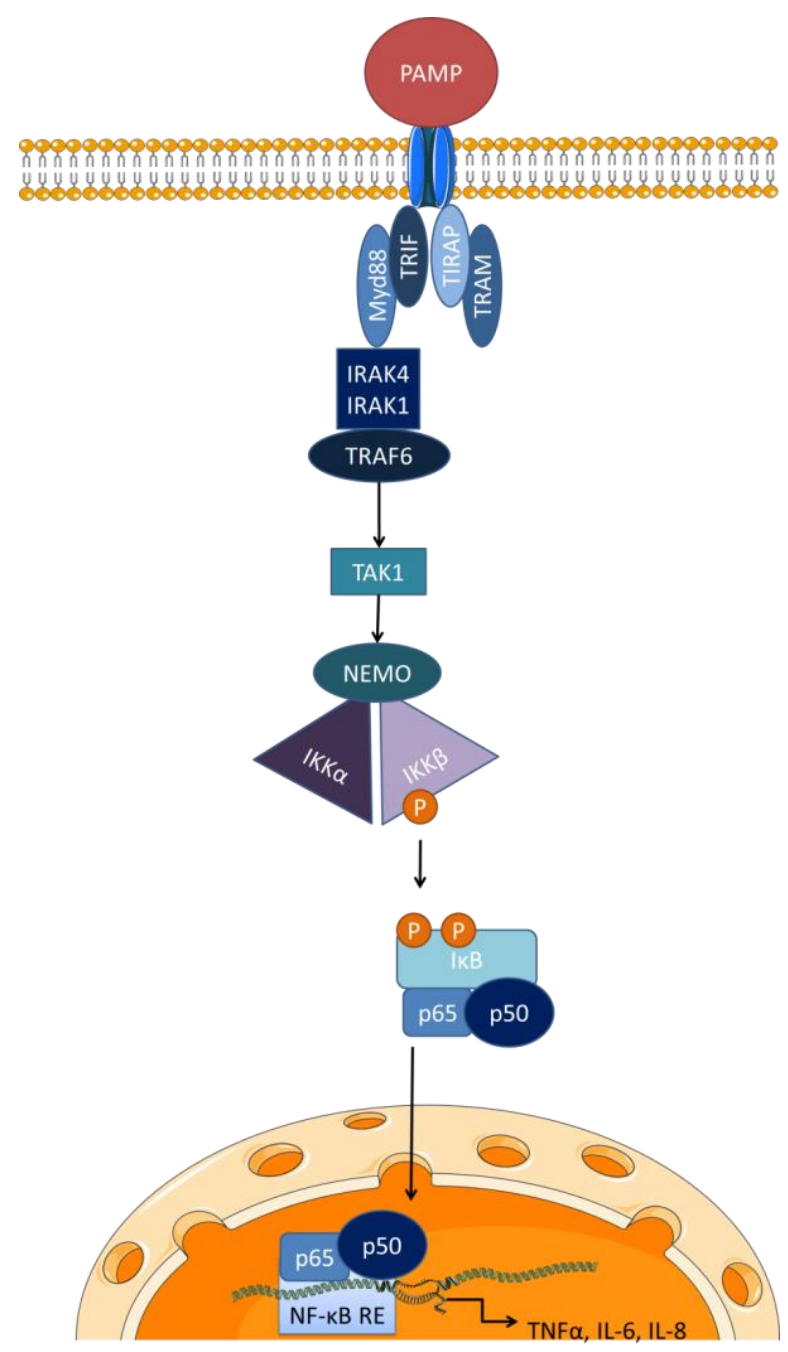

Figure 10: NF-kB pathway. PAMP = pathogen-associated molecular patterns; Myd88 = myeloid differentiation primary response gene 88; TRIF = TIR-domain-containing adapter-inducing interferon- $\beta$; TIRAP $=$ toll-interleukin 1 receptor (TIR) domain containing adaptor protein; TRAM = (TIR) domain-containing adaptor-inducing IFN- $\beta$ (TRIF)-related adaptor molecule; IRAK4/1 = IL-1R-associated kinase4/1; TRAF6 = TNF receptor-associated factor 6; TAK1 = transforming growth factor beta-activated kinase 1; NEMO = NF-кB essential modulator; IKK $\alpha=$ ІКB kinase $\alpha$; ІКК $\beta=$ ІКB kinase $\beta$; ІКB = inhibitor of $к B ;$ p50 = Nuclear factor NF-kappa-B p105 subunit; $\mathbf{p 6 5}=$ nuclear factor NFkappa-B p65 subunit; NF-KB RE = nuclear factor kappa $B$ response element; TNF $\alpha=$ tumor necrosis factor $\alpha$; IL-6 = interleukin-6; IL-8 = interleukin-8. 


\subsubsection{HIF}

HIF is a transcription factor that is activated during low oxygen levels ( $<1-2 \%$ of $\mathrm{O}_{2}$ ). It plays a major role in angiogenesis (105). HIF is a dimer that consists of two subunits: a HIF-1 $\beta$ subunit and either a HIF-1 $\alpha$, a HIF- $2 \alpha$ or a HIF-3 $\alpha$. At regular oxygen tension prolyl hydroxylases (PHDs) hydroxylate the proline residues present in HIF-1 $\alpha$ (Figure 11).
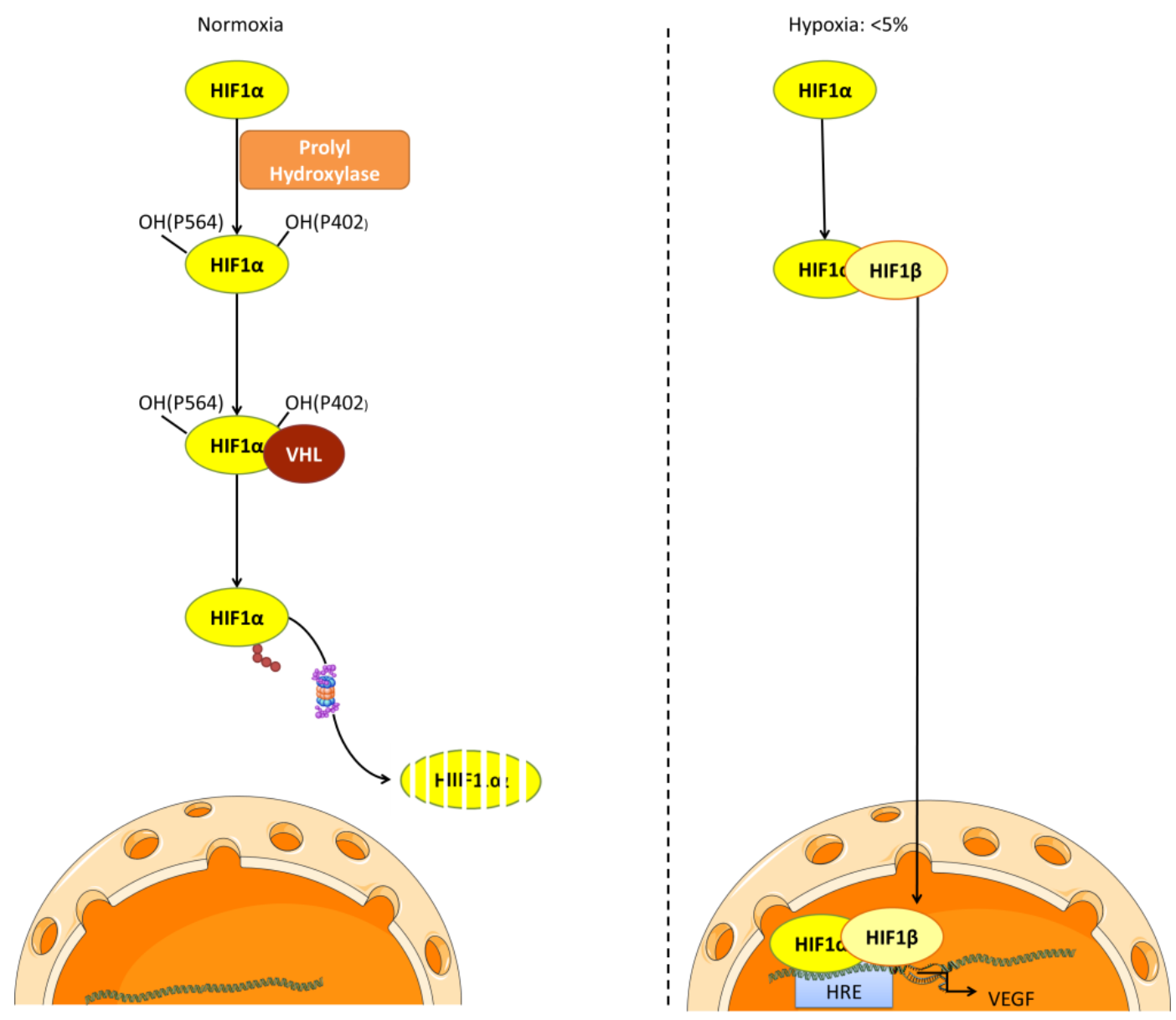

Figure 11: HIF pathway. HIF1 $\alpha=$ hypoxia-inducible factor 1-alpha; VHL = von Hippel Lindau; HIF1 $\beta$ = hypoxia-inducible factor 1 -beta; HRE = hypoxia response element; VEGF $=$ vascular endothelial growth factor.

Hydroxylation of HIF-1 $\alpha$ results in binding of the von Hippel-Lindau tumor suppressor protein ( $\mathrm{pVHL}$ ). $\mathrm{pVHL}$ induces proteasomal degradation of HIF-1 $\alpha$ by recruiting ubiquitin ligases. However, at low oxygen levels, PHDs are inactivated, leading to 
stabilization of HIF- $1 \alpha$ that forms a dimer with HIF-1 $\beta$ and translocates to the nucleus where it binds hypoxia response elements (HRE) that contain a (G/ACGTG) motif and are present in the promoter region of its target genes. One of its defined target genes is vascular endothelial growth factor (VEGF). Although it is not very common, HIF can also be activated in a hypoxia-independent way by certain growth factors, cytokines or hormones. Also in this pathway redox regulation can be performed at different levels, one example is the oxidation of $\mathrm{Fe}^{2+}$ into $\mathrm{Fe}^{3+}$ in $\mathrm{PHDs}$ by $\mathrm{H}_{2} \mathrm{O}_{2}$ resulting in the inactivation of the enzyme and activation of the HIF pathway (106).

\subsection{Cell death}

\subsubsection{GAPDH}

Glyceraldehyde-3-phosphate dehydrogenase (GAPDH) is a homotetrameric protein from which each subunit had a mass of $36 \mathrm{kDa}$. It also has four cysteine residues in each subunit. In humans this is cysteine 150, 154, 245 and 282. GAPDH is considered as a housekeeping gene. It is involved in the glycolysis and every cell needs to generate energy via the glycolysis, making GAPDH an essential enzyme in every cell. It catalyzes the generation of 1,3-biphosphoglycerate from glyceraldehyde-3-phosphate using nicotinamide adenine dinucleotide $\left(\mathrm{NAD}^{+}\right)$as cofactor, which is the sixth step of the glycolysis $(107,108)$ (Figure 12).

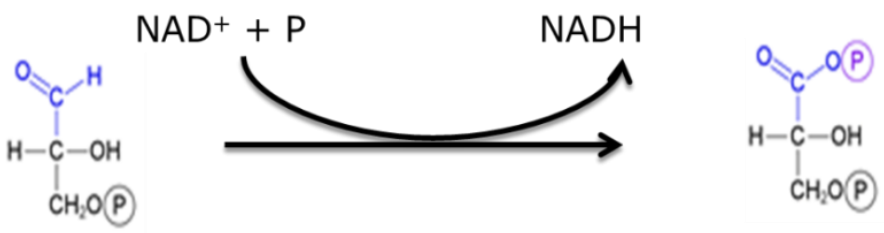

Figure 12: GAPDH reaction in glycolysis. GAPDH = glyceraldehyde-3-phosphate dehydrogenase; NADH = reduced nicotinamide adenine dinucleotide.

Next to its role in the glycolysis, GAPDH appears to be involved in many other uncommon functions. Therefore, it is called a 'moonlighting protein' (109). These 'moonlighting' functions include DNA repair and integrity, gene expression, ribonucleic acid (RNA) stabilization, cytoskeletal dynamics and transport, cell signaling and apoptosis. To induce a specific function, a tight regulation is essential. Oligomerization, posttranslational modifications and subcellular localizations play a major role in the regulation of the many functions of GAPDH. Oxidative stress appears to be a major regulator in the induction of these different functions (110). Especially the thiol groups are subject to oxidation. In case of a high level of oxidative stress, glutathionylation increases energy 
generation via the pentose phosphate pathway (111) (Figure 13). Via this way, the $\mathrm{NADPH} / \mathrm{NADP}^{+}$ratio remains in homeostasis. NADPH is an essential cofactor for many enzymes including glutathione peroxidase (GPx). By ensuring that GPx is provided with its cofactor, it ensures that GPx can covert reactive $\mathrm{H}_{2} \mathrm{O}_{2}$ into $\mathrm{H}_{2} \mathrm{O}$, thereby decreasing the damage induced by oxidative stress. During this oxidative modification, the glycolytic function of GAPDH is lost.

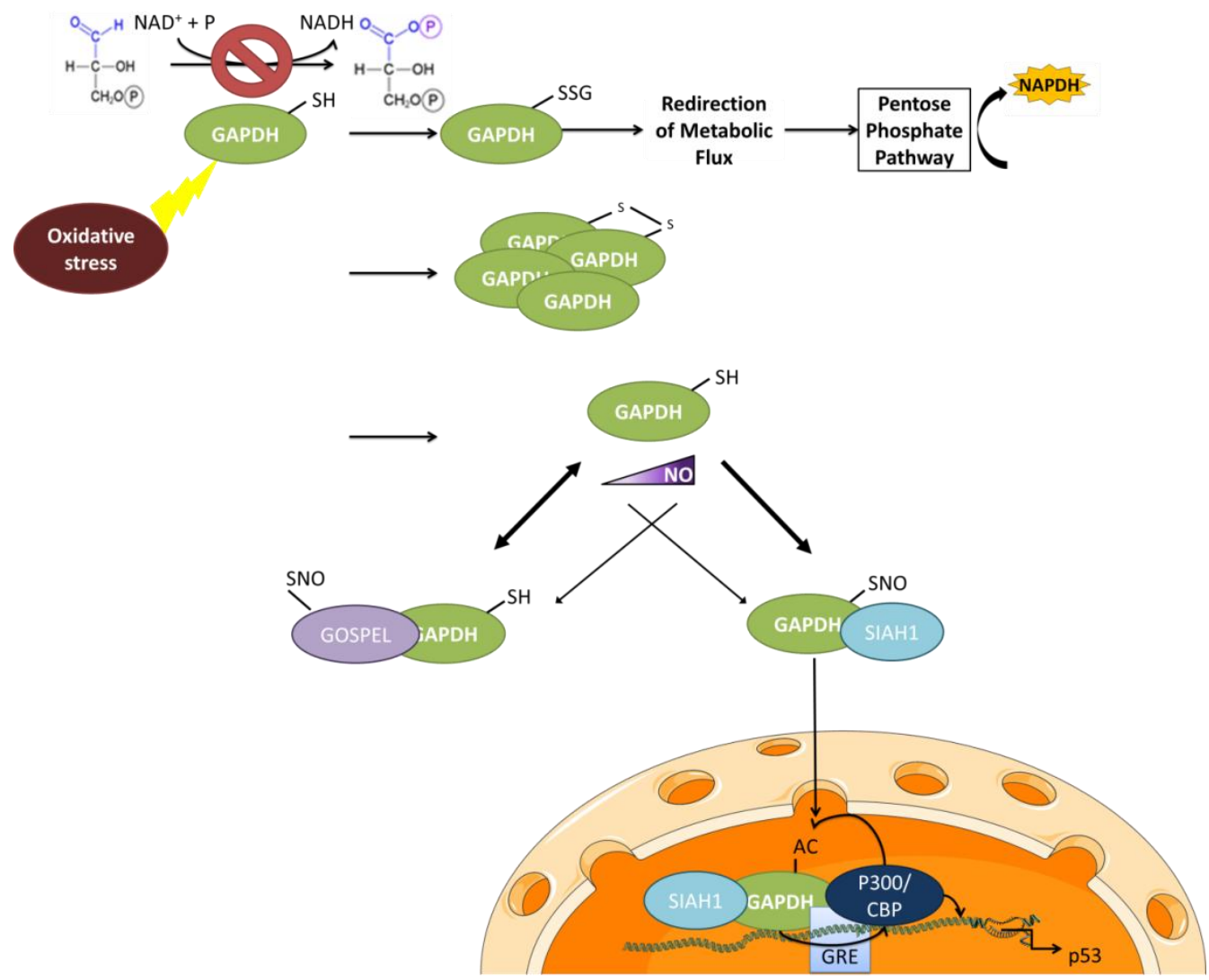

Figure 13: GAPDH pathway (110). GAPDH = glyceraldehyde-3-phosphate dehydrogenase; $\mathrm{NADH}=$ reduced nicotinamide adenine dinucleotide; $\mathrm{NADPH}=$ reduced nicotinamide adenine dinucleotide phosphate; NO = nitric oxide; GOSPEL = GAPDH's competitor of Siah Protein Enhances Life; SIAH1 = seven in absentia homolog 1; GRE = GAPDH response element; $p 300 / C B P=$ p300/ CREB-binding protein.

If GAPDH is exposed to oxidative stress, it can be nitrosylated. In basal circumstances, nitrosylation of Glyceraldehyde-3-phosphate dehydrogenase's (GAPDH's) competitor of Siah Protein Enhances Life (GOSPEL) occurs, a protein that binds GAPDH and 
keeps it in cytoplasmic surroundings. While at exposure to higher levels of nitrosative stress, GAPDH is nitrosylated next to GOSPEL. Now GAPDH does not bind to GOSPEL anymore, but interacts with seven in absentia homolog 1 (SIAH1) (77). GAPDH is now also subject to sulphination and sulphonation. Since Siah1 has a nuclear localization signal (NLS), the GAPDH-Siah complex is translocated to the nucleus (112). In the nucleus it functions as a transcription factor. GAPDH is acetylated by histone acetyltransferase p300/CBP. Acetylated GAPDH then enhances p300/CBP activity. Histon acetylation at tumor suppressor gene $\mathrm{p} 53$, results in loose packing of DNA and enhanced transcription of p53. This contributes to the initiation of programmed cell death or apoptosis. During these two processes especially cysteine 152 is targeted, the cysteine present in the catalytic site of GAPDH, thereby the glycolytic activity is impaired. A final oxidative modification of GAPDH is the formation of disulfide bonds between different GAPDH molecules and the aggregates (22). These aggregates can on their turn induce the unfolded protein response and via this way lead to the induction of apoptosis. These moonlighting roles of GAPDH are recently discovered, using GAPDH as a housekeeping gene should therefore be critically reconsidered. Nuclear translocation and associated apoptosis has been seen in diabetic retinopathy, additional the GAPDH aggregates have been shown to play a major role in the neurodegeneration process in Alzheimer's disease $(113,114)$.

\subsection{Oxidative stress-related diseases}

A good balance between ROS and antioxidants is essential (Figure 14A). ROS is necessary for redox signaling, but too much or too less ROS can lead to disease (115). In many diseases ROS plays a major role, but in this introduction, the role of ROS in the development of COPD, cardiovascular disease and cancer is elaborated.

\subsubsection{COPD}

COPD is characterised by the chronic bronchitis and emphysema $(116,117)$ and according to the global initiative of for chronic obstructive pulmonary disease it is defined as a preventable and treatable disease. Chronic bronchitis is established by bronchial airway thickening and mucus hyper secretion, most of all as a consequence of increased inflammation, especially an increase in neutrophils, macrophages and lymphocytes in particular $\mathrm{CD}^{+} \mathrm{T}$ lymphocytes is observed. The main features of emphysema are alveolar wall destruction, loss of elastic recoil in the alveoli and fibrosis, which impairs gas exchange between blood and air. This will all contribute to airflow limitation and an exaggerated immune response to non-dangerous gases or particles. The limitations in airflow usually only worsen and are in the end not fully reversible. This will lead to the characteristic symptoms of COPD, dyspnoea, chronic cough, chronic sputum production, 
wheezing and chest tightness. Based on these symptoms COPD is diagnosed. In addition, the frequency of exacerbations, acute worsening of the condition of the patient, comorbidities, and the diagnostic results from spirometry, can contribute to diagnosis of COPD. An acute worsening of the patient's condition often results in changes in medication and hospitalisation. COPD is classified in four global initiative for chronic obstructive lung disease (GOLD) categories: mild (A), moderate (B), severe (C) or very severe (D). Medical treatment starts with short-acting bronchodilators or long-acting bronchodilators. But in severe COPD, glucocorticoids are added. In very severe patients COPD oxygen therapy or surgical treatment are considered $(118,119)$.

The cause of COPD is still enigmatic. But oxidative stress has been defined as a central factor in the pathophysiology of $\operatorname{COPD}(111,120)$. Various exogenous and endogenous sources contribute to the development of COPD. Smoking is the most important exogenous source of COPD, because almost $90 \%$ of the COPD patients smokes (121). Next to smoking, airborne pollution, car exhaust fumes and indoor cooking fires are important environmental sources of oxidant generation. Next to exogenous, also endogenous sources contribute to the increased oxidant/antioxidant balance in COPD (3). First of all, endogenous mitochondrial metabolism attributes to the development of COPD (3). Additionally, immune cells like neutrophils and macrophages are recruited and also structural cells including epithelial and endothelial cells function as important ROS generators. Due to the presence of NADPH oxidase and xanthine oxidase, neutrophils are able to generate $\mathrm{H}_{2} \mathrm{O}_{2}$ that normally functions to combat a microbial threat $(3,122)$. Therefore, bacteria and viruses are the main factor inducing an exacerbation. Due to an impaired innate lung defense, bacterial colonization is triggered. Subsequently, they enhance inflammation, which leads to an impaired proteinase/antiproteinase balance in favour of the proteinases, and an even more increase of oxidative stress. This will contribute to disease progression by resulting in airway epithelial damage, permeability and remodeling. ROS is not always detrimental, since in order to induce a good immune response a specific amount of ROS is necessary. For example, normally the innate immune system is activated when bacterial antigens, called PAMPs or damage-associated molecular patterns (DAMPs) bind to the toll-like receptor (TLR) that activates downstream NADPH oxidases (NOX) that produce ROS including $\mathrm{H}_{2} \mathrm{O}_{2}$ which lead to NF-kB-mediated release of pro-inflammatory cytokines like TNF $\alpha$, interferon beta (IFN $\beta$ ) and interleukin1 beta (IL-1B) (64). When the level of ROS is too low, this will lead to immunosuppression, however, when the level of ROS is too high, this will enhance the inflammatory response (Figure 14B) $(64,115)$. 
A. Basal conditions

Protection

Toxicity

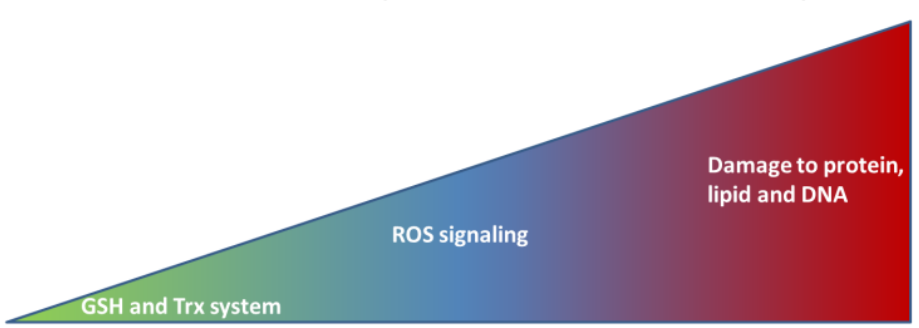

B. Inflammation

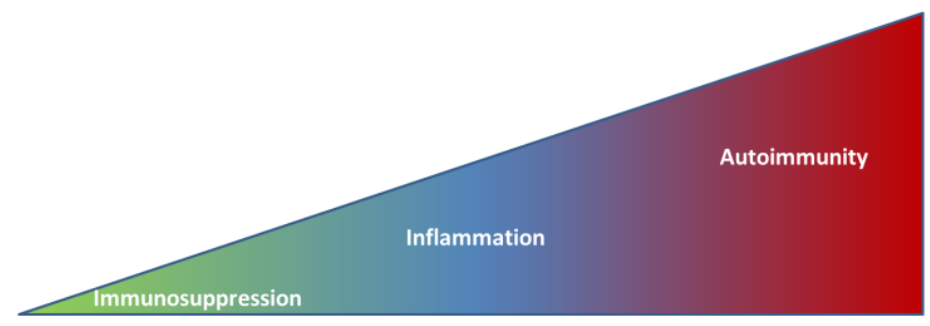

\section{Cancer}

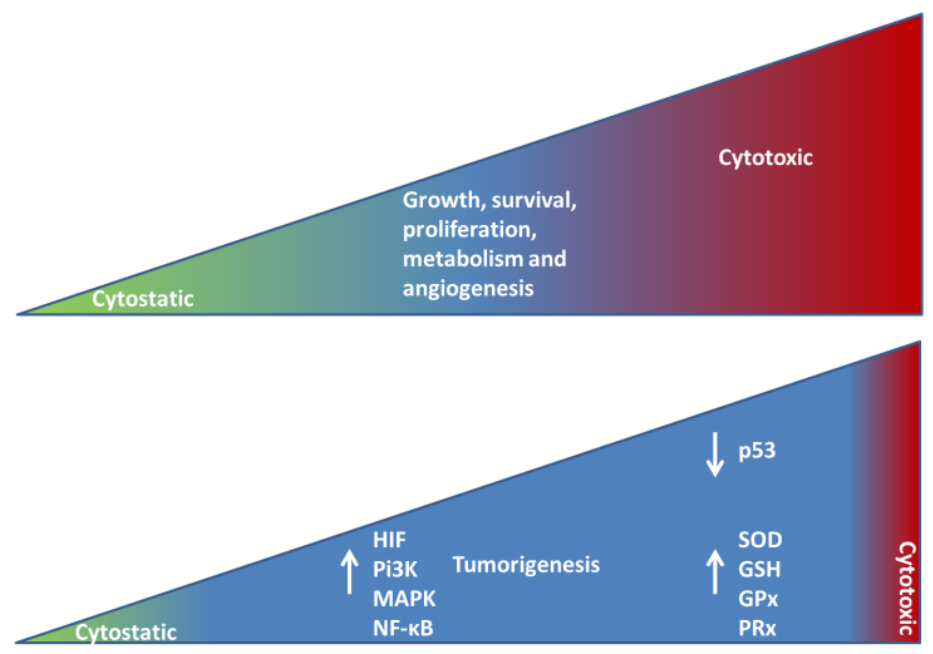

Figure 14: The effect of ROS in normal ROS signaling (A), inflammation (B) and cancer (C), adapted from Schieber et al. (64) and Galadari et al. (115). GSH = glutathione; Trx = thioredoxin; ROS = reactive oxygen species; DNA = deoxyribonucleic acid; HIF = hypoxia inducible factor; $\mathrm{Pi} 3 \mathrm{~K}=$ phosphoinositide 3-kinase; $\mathrm{MAPK}=$ mitogen-activated protein kinase; NF-кB = nuclear factor kappa B; SOD = superoxide dismutase; GPx = glutathione peroxidase; $\mathbf{P R x}=$ peroxiredoxin. 
In this case, this will lead to carbonyl stress and tissue damage. As a consequence of carbonyl stress, many pathophysiologic mechanisms associated with COPD are induced including mitochondrial damage, autoimmune induction, pro-inflammatory signaling, mucus hypersecretion, bronchial constriction, DNA damage, proliferation of specific adaptive immune cells, tissue remodeling, muscle dysfunction, impaired phagocytic and steroid function (123).

\subsubsection{Cardiovascular disease}

CVD is not one disease but a group of diseases that influences the heart and blood vessels. CVD related to the heart are cardiomyopathy, hypertensive heart disease, heart failure, pulmonary heart disease, cardiac dysrhythmias, inflammatory heart disease including endo- or myocarditis, valvular heart disease, congenital heart disease or rheumatic heart disease (124). The types of CVD related to the vessels are coronary artery disease, peripheral arterial disease, cerebrovascular disease including stroke, renal artery stenosis or aortic aneurysm (124). In coronary artery disease, stroke and peripheral artery disease the pathophysiological mechanism of atherosclerosis plays an important role. Symptoms are discomfort in chest or arm region, confusion, difficulty with seeing or walking, severe headache or unconsciousness (124). Risk factors of developing CVD are higher age, smoking, physical inactivity, obesity, increased blood glucose/diabetes, increased blood cholesterol/hyperlipidemia, excessive alcohol use, genetic predisposition, high blood pressure/hypertension, chronic kidney problems, psychosocial and educational factors (124). Diagnosis is mainly done by screening on blood lipids, glucose levels, hypertension or making an electrocardiogram (ECG) in case of suspicion (124). Treatment includes pharmaceutical medication including anticoagulants like aspirin; antihypertensive mediation, diuretics, renin-angiotensin-aldosterone system (RAAS)-inhibitors, beta blockers or calcium antagonists; antiarrhythmic agents, nitrates, positive inotropic agents or antilipidemic agents like statins. On the long term sometimes surgery is required. If the medication is not sufficient coronary artery bypass, balloon angioplasty, valve repair and replacement, pacemaker devices or heart transplantation can be considered $(124,125)$. In the development of CVD, oxidative stress plays a major role. The various risk factors can stimulate the sympathic nerve system leading to increased norepinephrine, activate the RAAS system resulting in increased angiotensin II, induce endothelial dysfunction associated with increased peroxynitrite radical formation, activate the immune system in particular neutrophils that release radicals or ROS like hypochlorous acid (126). All these pathophysiological mechanisms increase oxidative stress and lead to cardiac defects including heart dysfunction or arrhythmias or vascular defects like hypertension or atherosclerosis. A short increase is again necessary for ROS signaling, however prolonged exposure to ROS leads to damage to sarcolemma, sarcoplasmic reticulum, mitochondria, 
myofibrils or nucleus leading to abnormalities in calcium signaling, disruption in energy stores and DNA fragmentation that finally contribute to myocyte dysfunction (126).

\subsubsection{Cancer}

Cancer is also not one disease, but a group of diseases. Depending on the primary tumor, the tissue where the cancer starts, different types of cancer exists. First of all, cancer starting in epithelial cells is called carcinoma (127). Sarcoma is cancer derived from connective tissue, while lymphoma or leukemia arises in hematopoietic cells. Finally germ cell cancer and blastoma exists, wherein the first arises in germ cells and the second can mainly be found in embryos (127). Local symptoms are pain or bleeding, a systemic symptom is unintentional weight loss, and depending on the disease progression, also metastasis can occur, spread of cancer cells to other parts of the body if this is in the lymphoic tissue, this can result in swollen lymph nodes. Diagnosis is mainly done via blood tests or X-rays, computed tomography (CT) scans or endoscopy (127). The main treatments against cancer are chemotherapy, radiation or surgery (127). However, currently research is focused more on developing more specific immunotherapy or other more targeted treatments. The cause on developing cancer is still enigmatic, although genetics, various chemical reagents and diet can have a huge contribution to the development and progression of the disease. The pathophysiological cellular and molecular mechanisms are defined by Hanahan and Weinberg as the hallmarks of cancer. The hallmarks are resisting cell death, sustaining proliferative signaling, evading growth suppressors, inducing angiogenesis, enabling replicative immortality, activating invasion and metastasis, deregulating cellular energetics, avoiding immune destruction, genome instability, genome mutation and tumor-promoting inflammation (128). Also in processes such as metabolism and angiogenesis, growth, survival an proliferation, ROS signaling is necessary for activation of HIF, NF-KB, Pi3K and MAPK (64) (Figure 14C) (64, 115). Decreased levels or ROS result in no growth and proliferation and lead to cytostatic effects. However, during tumorigenesis, genetic changes in HIF, NF-KB, Pi3K and MAPK, now called oncogenes, hypoxia and altered metabolism lead to consistently increased ROS levels (64). This enhances continuously activated proliferation, survival and metabolic responses (64). Increased endogenous antioxidant systems such as the GSH and Trx system, but also enzymes like catalase and superoxide dismutases, will prevent tumor cell death or cytotoxicity due to oxidative stress (64). Concomitantly with a decreased induction of cell death, because of genetic modification of tumor suppressor genes including p53 that induces programmed cell death, this will further contribute to tumorigenesis (64). 


\subsection{Hormesis}

In toxicology, hormesis can be defined as the ability of an organism to adapt by inducing endogenous protective mechanisms upon exposure to a low dose of a specific compound, thereby protecting itself against subsequent (toxic) exposures of this compound (129). Many definitions and names have been given to this phenomenon over time. First the concept homeostasis was introduced, meaning that variables are maintained within specific limits leading to a relative constant internal environment. Later the term heterostasis or allostasis are mentioned, indicative of a situation when homeostasis, the constant internal state, is disrupted. In addition also the word adaptation is often used, in my opinion, based on literature, this is the protective process induced upon treatment to a low dose of the respective compound. Recently, Davies even introduced the term adaptive homeostasis describing a situation in which the internal state is adapted and the borders of internal state are expanded or changed (130).

Hormesis does not only offer protection against future challenges with the same chemical, when two substances induce similar protective mechanisms, exposure to a low dose of one of the compounds offers protection against future challenges with the other chemical, this phenomenon is called transhormesis (45). If the compound that induces hormesis is generally accepted as a non-dangerous chemical, the process is defined as parahormesis $(52,131)$.

There are many requirements before the process of hormesis can be induced. The induction of hormesis is dependent on time, dose and the compound involved. Additionally, also different adaptive responses can be induced with consequences on short or long term (132). A detailed disquisition of hormesis and its contributing factors is shown in Chapter 2 of this thesis.

\section{Aims of thesis}

In this thesis the cellular response to toxins is studied, that comprises protection, adaptation and death. It is aimed to examine the concepts of hormesis and adaptation. Factors that influence hormesis are elaborated with a special focus on time, also taking the chemical nature of the compound of interest as well as the dose into account (Chapter 2). Next, the hormesis concept is materialized for acrolein, AgNPs, rutin and quercetin (Chapter 3, 4, 5, 7 respectively). Short term and long term protective and adaptive effects on the GSH and Trx systems are determined. The common denominator is that all compounds are thiol reactive, an activity that is crucial in the observed effect. The role of the Keap1-Nrf2 pathway, in the adaptive responses is further examined. For rutin, its 
cardiovascular health benefit is elaborated, namely, the protective effect of rutin on human arterioles is explained (Chapter $\mathbf{5}$ ). The interplay between compounds regarding the adaptive responses is described in Chapter 4. Finally, the studied biochemical mechanism that forms the thin line between adaptation and toxicity is studied in Chapter 6 and Chapter 7. In Chapter 6 the dual effect of acrolein on a specific target i.e. MGST1 is examined. Depending on the dose and time, acrolein can either enhance or diminish the protective potency of MGST1. To conclude, in Chapter $\mathbf{7}$ it is shown that the response to a chemical i.e. adaptation or cell death, can depend on the redox state of the cell. A whole new application of an already existing pathway in the range of protection, adaptation and toxicity is illustrated. Here the aim is not protection and adaptation, but toxicity. The eventual beneficial effect of toxicity will be further explained. 


\section{References}

1. Guiney SJ, Adlard PA, Bush Al, Finkelstein DI, Ayton S. Ferroptosis and cell death mechanisms in Parkinson's disease. Neurochem Int. 2017 Mar;104:34-48.

2. Moghe A, Ghare S, Lamoreau B, Mohammad M, Barve S, McClain C, et al. Molecular mechanisms of acrolein toxicity: relevance to human disease. Toxicological sciences : an official journal of the Society of Toxicology. 2015 Feb;143(2):242-55.

3. Rahman I. Oxidative stress in pathogenesis of chronic obstructive pulmonary disease: cellular and molecular mechanisms. Cell Biochem Biophys. 2005;43(1):167-88.

4. Bein K, Leikauf GD. Acrolein - a pulmonary hazard. Molecular nutrition \& food research. 2011 Sep;55(9):1342-60.

5. Woodruff TJ, Wells EM, Holt EW, Burgin DE, Axelrad DA. Estimating risk from ambient concentrations of acrolein across the United States. Environ Health Perspect. 2007 Mar;115(3):410-5.

6. Faroon O, Roney N, Taylor J, Ashizawa A, Lumpkin MH, Plewak DJ. Acrolein health effects. Toxicol Ind Health. 2008 Aug;24(7):447-90.

7. Struve MF, Wong VA, Marshall MW, Kimbell JS, Schroeter JD, Dorman DC. Nasal uptake of inhaled acrolein in rats. Inhal Toxicol. 2008 Feb;20(3):217-25.

8. Stevens JF, Maier CS. Acrolein: sources, metabolism, and biomolecular interactions relevant to human health and disease. Molecular nutrition \& food research. 2008 Jan;52(1):7-25.

9. Lopachin RM, Gavin T, Decaprio A, Barber DS. Application of the Hard and Soft, Acids and Bases (HSAB) theory to toxicant--target interactions. Chemical research in toxicology. 2012 Feb 20;25(2):239-51.

10. LoPachin RM, Gavin T, Petersen DR, Barber DS. Molecular mechanisms of 4-hydroxy-2-nonenal and acrolein toxicity: nucleophilic targets and adduct formation. Chemical research in toxicology. 2009 Sep;22(9):1499-508.

11. Witz G. Biological interactions of alpha,beta-unsaturated aldehydes. Free radical biology \& medicine. 1989;7(3):333-49.

12. Cai J, Bhatnagar A, Pierce WM, Jr. Protein modification by acrolein: formation and stability of cysteine adducts. Chemical research in toxicology. 2009 Apr;22(4):708-16. 
13. Esterbauer H, Schaur RJ, Zollner H. Chemistry and biochemistry of 4hydroxynonenal, malonaldehyde and related aldehydes. Free radical biology \& medicine. 1991;11(1):81-128.

14. Wang $\mathrm{HT}$, Zhang $\mathrm{S}, \mathrm{Hu} \mathrm{Y}$, Tang MS. Mutagenicity and sequence specificity of acrolein-DNA adducts. Chemical research in toxicology. 2009 Mar 16;22(3):5117.

15. Ishii T, Yamada T, Mori T, Kumazawa S, Uchida K, Nakayama T. Characterization of acrolein-induced protein cross-links. Free Radic Res. 2007 Nov;41(11):1253-60.

16. Heim KE, Tagliaferro AR, Bobilya DJ. Flavonoid antioxidants: chemistry, metabolism and structure-activity relationships. J Nutr Biochem. 2002 Oct;13(10):572-84.

17. Nishimuro $H$, Ohnishi $H$, Sato $M$, Ohnishi-Kameyama $M$, Matsunaga I, Naito $S$, et al. Estimated daily intake and seasonal food sources of quercetin in Japan. Nutrients. 2015 Apr 02;7(4):2345-58.

18. Egert S, Wolffram S, Bosy-Westphal A, Boesch-Saadatmandi C, Wagner AE, Frank J, et al. Daily quercetin supplementation dose-dependently increases plasma quercetin concentrations in healthy humans. The Journal of nutrition. 2008 Sep;138(9):1615-21.

19. Sakac M, Pestoric M, Misan A, Nedeljkovic N, Jambrec D, Jovanov P, et al. Antioxidant Capacity, Mineral Content and Sensory Properties of Gluten-Free Rice and Buckwheat Cookies. Food Technol Biotechnol. 2015 Mar;53(1):38-47.

20. Kumar S, Pandey AK. Chemistry and biological activities of flavonoids: an overview. ScientificWorldJournal. 2013;2013:162750.

21. Jacobs $H$, Moalin $M$, van Gisbergen MW, Bast A, van der Vijgh WJ, Haenen GR. An essential difference in the reactivity of the glutathione adducts of the structurally closely related flavonoids monoHER and quercetin. Free radical biology \& medicine. 2011 Dec 01;51(11):2118-23.

22. Jacobs $H$, Moalin $M$, Bast $A$, van der Vijgh WJ, Haenen GR. An essential difference between the flavonoids monoHER and quercetin in their interplay with the endogenous antioxidant network. PLoS One. 2010 Nov 08;5(11):e13880.

23. de Boer VC, Dihal AA, van der Woude H, Arts IC, Wolffram S, Alink GM, et al. Tissue distribution of quercetin in rats and pigs. J Nutr. 2005 Jul;135(7):1718-25. 
24. Sengupta B, Sengupta PK. The interaction of quercetin with human serum albumin: a fluorescence spectroscopic study. Biochemical and biophysical research communications. 2002 Dec 06;299(3):400-3.

25. Mullen W, Edwards CA, Crozier A. Absorption, excretion and metabolite profiling of methyl-, glucuronyl-, glucosyl- and sulpho-conjugates of quercetin in human plasma and urine after ingestion of onions. Br J Nutr. 2006 Jul;96(1):10716.

26. Yeh $\mathrm{SL}$, Lin $\mathrm{YC}$, Lin $\mathrm{YL}$, Li CC, Chuang $\mathrm{CH}$. Comparing the metabolism of quercetin in rats, mice and gerbils. Eur J Nutr. 2016 Feb;55(1):413-22.

27. Manach C, Williamson G, Morand C, Scalbert A, Remesy C. Bioavailability and bioefficacy of polyphenols in humans. I. Review of 97 bioavailability studies. The American journal of clinical nutrition. 2005 Jan;81(1 Suppl):230S-42S.

28. Carbonaro $M$, Grant $G$. Absorption of quercetin and rutin in rat small intestine. Ann Nutr Metab. 2005 May-Jun;49(3):178-82.

29. Manach C, Scalbert A, Morand C, Remesy C, Jimenez L. Polyphenols: food sources and bioavailability. The American journal of clinical nutrition. 2004 May;79(5):727-47.

30. Sharma S, Ali A, Ali J, Sahni JK, Baboota S. Rutin : therapeutic potential and recent advances in drug delivery. Expert opinion on investigational drugs. 2013 Aug;22(8):1063-79.

31. Nait Chabane M, Al Ahmad A, Peluso J, Muller CD, Ubeaud G. Quercetin and naringenin transport across human intestinal Caco-2 cells. J Pharm Pharmacol. 2009 Nov;61(11):1473-83.

32. Walle T, Vincent TS, Walle UK. Evidence of covalent binding of the dietary flavonoid quercetin to DNA and protein in human intestinal and hepatic cells. Biochemical pharmacology. 2003 May 15;65(10):1603-10.

33. Walgren RA, Lin JT, Kinne RK, Walle T. Cellular uptake of dietary flavonoid quercetin 4'-beta-glucoside by sodium-dependent glucose transporter SGLT1. J Pharmacol Exp Ther. 2000 Sep;294(3):837-43.

34. Lemmens KJ, van de Wier B, Vaes N, Ghosh M, van Zandvoort MA, van der Vijgh WJ, et al. The flavonoid 7-mono-O-(beta-hydroxyethyl)-rutoside is able to protect endothelial cells by a direct antioxidant effect. Toxicology in vitro : an international journal published in association with BIBRA. 2014 Jun;28(4):538-43.

35. Lemmens KJ, Vrolijk MF, Bouwman FG, van der Vijgh WJ, Bast A, Haenen $\mathrm{GR}$. The minor structural difference between the antioxidants quercetin and 4 'O- 
methylquercetin has a major impact on their selective thiol toxicity. International journal of molecular sciences. 2014 Apr 30;15(5):7475-84.

36. Boots AW, Balk JM, Bast A, Haenen GR. The reversibility of the glutathionyl-quercetin adduct spreads oxidized quercetin-induced toxicity. Biochemical and biophysical research communications. 2005 Dec 16;338(2):923-9. 37. Boots AW, Li H, Schins RP, Duffin R, Heemskerk JW, Bast A, et al. The quercetin paradox. Toxicology and applied pharmacology. 2007 Jul 01;222(1):8996.

38. Bast A, Haenen GR. Ten misconceptions about antioxidants. Trends Pharmacol Sci. 2013 Aug;34(8):430-6.

39. Jacobs $H$, van der Vijgh WJ, Koek GH, Draaisma GJ, Moalin $M$, van Strijdonck GP, et al. Characterization of the glutathione conjugate of the semisynthetic flavonoid monoHER. Free radical biology \& medicine. 2009 Jun 15;46(12):1567-73.

40. Behra R, Sigg L, Clift MJ, Herzog F, Minghetti M, Johnston B, et al. Bioavailability of silver nanoparticles and ions: from a chemical and biochemical perspective. J R Soc Interface. 2013 Oct 06;10(87):20130396.

41. Reidy B, Haase A, Luc A. Mechanisms of Silver Nanoparticle Release, Transformation and Toxicity: A Critical Review of Current Knowledge and Recommendations for Future Studies and Applications Materials. 2013;6:2295350 .

42. Tulve NS, Stefaniak AB, Vance ME, Rogers K, Mwilu S, LeBouf RF, et al. Characterization of silver nanoparticles in selected consumer products and its relevance for predicting children's potential exposures. Int J Hyg Environ Health. 2015 May;218(3):345-57.

43. Kermanizadeh A, Vranic S, Boland S, Moreau K, Baeza-Squiban A, Gaiser $B K$, et al. An in vitro assessment of panel of engineered nanomaterials using a human renal cell line: cytotoxicity, pro-inflammatory response, oxidative stress and genotoxicity. BMC Nephrol. 2013 Apr 25;14:96.

44. Georgantzopoulou A, Balachandran YL, Rosenkranz P, Dusinska M, Lankoff $A$, Wojewodzka $M$, et al. Ag nanoparticles: size- and surface-dependent effects on model aquatic organisms and uptake evaluation with NanoSIMS. Nanotoxicology. 2013 Nov;7(7):1168-78. 
45. Sthijns MM, Thongkam W, Albrecht C, Hellack B, Bast A, Haenen GR, et al. Silver nanoparticles induce hormesis in A549 human epithelial cells. Toxicology in vitro : an international journal published in association with BIBRA. 2017 Jan 18.

46. Yildirimer L, Thanh NT, Loizidou M, Seifalian AM. Toxicology and clinical potential of nanoparticles. Nano Today. 2011 Dec;6(6):585-607.

47. Sung JH, Ji JH, Park JD, Yoon JU, Kim DS, Jeon KS, et al. Subchronic inhalation toxicity of silver nanoparticles. Toxicological sciences : an official journal of the Society of Toxicology. 2009 Apr;108(2):452-61.

48. 2015; Available from: https://toxnet.nlm.nih.gov/cgibin/sis/search/a?dbs+hsdb:@term+@DOCNO+7715.

49. Tang J, Xiong L, Wang S, Wang J, Liu L, Li J, et al. Distribution, translocation and accumulation of silver nanoparticles in rats. J Nanosci Nanotechnol. 2009 Aug;9(8):4924-32.

50. Aueviriyavit S, Phummiratch D, Maniratanachote R. Mechanistic study on the biological effects of silver and gold nanoparticles in Caco-2 cells--induction of the Nrf2/HO-1 pathway by high concentrations of silver nanoparticles. Toxicol Lett. 2014 Jan 03;224(1):73-83.

51. Dziendzikowska K, Gromadzka-Ostrowska J, Lankoff A, Oczkowski M, Krawczynska A, Chwastowska J, et al. Time-dependent biodistribution and excretion of silver nanoparticles in male Wistar rats. J Appl Toxicol. 2012 Nov;32(11):920-8.

52. Ursini F, Maiorino M, Forman HJ. Redox homeostasis: The Golden Mean of healthy living. Redox biology. 2016 Aug;8:205-15.

53. Galli F, Piroddi M, Annetti C, Aisa C, Floridi E, Floridi A. Oxidative stress and reactive oxygen species. Contrib Nephrol. 2005;149:240-60.

54. Halliwell B, Gutteridge JMC. Free Radicals in Biology and Medicine. 5 ed: Oxford University Press; 2015.

55. Giorgio $M$, Trinei $M$, Migliaccio E, Pelicci PG. Hydrogen peroxide: a metabolic by-product or a common mediator of ageing signals? Nat Rev Mol Cell Biol. 2007 Sep;8(9):722-8.

56. Hall CN, Garthwaite J. What is the real physiological NO concentration in vivo? Nitric Oxide. 2009 Sep;21(2):92-103.

57. Adly AAM. Oxidative stress and disease: an updated review Res J Immunolog 2010;3(2):129-45. 
58. Al-Dalaen SM, Al-Qtaitat Al. Review article: Oxidative stress versus antioxidants. American Journal of Bioscience and Bioengineering. 2014;2(5):60-71. 59. Paulsen CE, Carroll KS. Cysteine-mediated redox signaling: chemistry, biology, and tools for discovery. Chem Rev. 2013 Jul 10;113(7):4633-79.

60. Ray PD, Huang BW, Tsuji Y. Reactive oxygen species (ROS) homeostasis and redox regulation in cellular signaling. Cell Signal. 2012 May;24(5):981-90.

61. Forman $\mathrm{HJ}$, Maiorino $\mathrm{M}$, Ursini F. Signaling functions of reactive oxygen species. Biochemistry. 2010 Feb 09;49(5):835-42.

62. Saccoccia F, Angelucci $F$, Boumis G, Desiato G, Miele AE, Bellelli A. Selenocysteine robustness versus cysteine versatility: a hypothesis on the evolution of the moonlighting behaviour of peroxiredoxins. Biochem Soc Trans. 2014 Dec;42(6):1768-72.

63. Jensen KS, Pedersen JT, Winther JR, Teilum K. The pKa value and accessibility of cysteine residues are key determinants for protein substrate discrimination by glutaredoxin. Biochemistry. 2014 Apr 22;53(15):2533-40.

64. Schieber M, Chandel NS. ROS function in redox signaling and oxidative stress. Curr Biol. 2014 May 19;24(10):R453-62.

65. Rahal A, Kumar A, Singh V, Yadav B, Tiwari R, Chakraborty S, et al. Oxidative stress, prooxidants, and antioxidants: the interplay. Biomed Res Int. 2014;2014:761264.

66. Griffiths HR, Moller L, Bartosz G, Bast A, Bertoni-Freddari C, Collins A, et al. Biomarkers. Mol Aspects Med. 2002 Feb-Jun;23(1-3):101-208.

67. Finkel T, Holbrook NJ. Oxidants, oxidative stress and the biology of ageing. Nature. 2000 Nov 09;408(6809):239-47.

68. Lushchak VI. Glutathione homeostasis and functions: potential targets for medical interventions. J Amino Acids. 2012;2012:736837.

69. Forman HJ, Zhang H, Rinna A. Glutathione: overview of its protective roles, measurement, and biosynthesis. Mol Aspects Med. 2009 Feb-Apr;30(1-2):1-12.

70. Rahman I, MacNee W. Lung glutathione and oxidative stress: implications in cigarette smoke-induced airway disease. Am J Physiol. 1999 Dec;277(6 Pt 1):L1067-88.

71. Carilho Torrao RB, Dias IH, Bennett SJ, Dunston CR, Griffiths HR. Healthy ageing and depletion of intracellular glutathione influences $\mathrm{T}$ cell membrane thioredoxin-1 levels and cytokine secretion. Chem Cent J. 2013 Sep 05;7(1):150. 
72. Ghezzi P. Protein glutathionylation in health and disease. Biochimica et biophysica acta. 2013 May;1830(5):3165-72.

73. Rinaldi R, Eliasson E, Swedmark S, Morgenstern R. Reactive intermediates and the dynamics of glutathione transferases. Drug Metab Dispos. 2002 Oct;30(10):1053-8.

74. Brigelius-Flohe R, Maiorino M. Glutathione peroxidases. Biochimica et biophysica acta. 2013 May;1830(5):3289-303.

75. Lillig $\mathrm{CH}$, Berndt $\mathrm{C}$, Holmgren A. Glutaredoxin systems. Biochimica et biophysica acta. 2008 Nov;1780(11):1304-17.

76. Soderberg A, Sahaf B, Rosen A. Thioredoxin reductase, a redox-active selenoprotein, is secreted by normal and neoplastic cells: presence in human plasma. Cancer Res. 2000 Apr 15;60(8):2281-9.

77. Di Trapani G, Perkins A, Clarke F. Production and secretion of thioredoxin from transformed human trophoblast cells. Mol Hum Reprod. 1998 Apr;4(4):36975.

78. Marino SM. Protein flexibility and cysteine reactivity: influence of mobility on the H-bond network and effects on pKa prediction. Protein J. 2014 Aug;33(4):323-36.

79. Cebula M, Schmidt EE, Arner ES. TrxR1 as a potent regulator of the Nrf2Keap1 response system. Antioxidants \& redox signaling. 2015 Oct 01;23(10):82353.

80. Arner ES, Holmgren A. Physiological functions of thioredoxin and thioredoxin reductase. Eur J Biochem. 2000 Oct;267(20):6102-9.

81. Zhong L, Arner ES, Holmgren A. Structure and mechanism of mammalian thioredoxin reductase: the active site is a redox-active selenolthiol/selenenylsulfide formed from the conserved cysteine-selenocysteine sequence. Proc Natl Acad Sci U S A. 2000 May 23;97(11):5854-9.

82. Anestal K, Prast-Nielsen S, Cenas N, Arner ES. Cell death by SecTRAPs: thioredoxin reductase as a prooxidant killer of cells. PLoS One. $2008 \mathrm{Apr}$ 02;3(4):e1846.

83. Kansanen E, Kuosmanen SM, Leinonen H, Levonen AL. The Keap1-Nrf2 pathway: Mechanisms of activation and dysregulation in cancer. Redox biology. 2013 Jan 18;1:45-9.

84. Itoh K, Chiba T, Takahashi S, Ishii T, Igarashi K, Katoh Y, et al. An $\mathrm{Nrf2/small} \mathrm{Maf}$ heterodimer mediates the induction of phase II detoxifying 
enzyme genes through antioxidant response elements. Biochemical and biophysical research communications. 1997 Jul 18;236(2):313-22.

85. Choo YY, Hagen T. Mechanism of cullin3 E3 ubiquitin ligase dimerization. PLoS One. 2012;7(7):e41350.

86. Itoh K, Wakabayashi N, Katoh Y, Ishii T, Igarashi K, Engel JD, et al. Keap1 represses nuclear activation of antioxidant responsive elements by Nrf2 through binding to the amino-terminal Neh2 domain. Genes Dev. 1999 Jan 01;13(1):76-86. 87. Zipper LM, Mulcahy RT. The Keap1 BTB/POZ dimerization function is required to sequester Nrf2 in cytoplasm. J Biol Chem. 2002 Sep 27;277(39):3654452.

88. Magesh S, Chen $\mathrm{Y}$, Hu L. Small molecule modulators of Keap1-Nrf2-ARE pathway as potential preventive and therapeutic agents. Med Res Rev. 2012 Jul;32(4):687-726.

89. D'Autreaux B, Toledano MB. ROS as signalling molecules: mechanisms that generate specificity in ROS homeostasis. Nat Rev Mol Cell Biol. 2007 Oct;8(10):813-24.

90. Lo SC, Li X, Henzl MT, Beamer L, Hannink M. Structure of the Keap1:Nrf2 interface provides mechanistic insight into Nrf2 signaling. EMBO J. 2006 Aug 09;25(15):3605-17.

91. Wang X, Tomso DJ, Chorley BN, Cho HY, Cheung VG, Kleeberger SR, et al. Identification of polymorphic antioxidant response elements in the human genome. Hum Mol Genet. 2007 May 15;16(10):1188-200.

92. Watai Y, Kobayashi A, Nagase H, Mizukami M, McEvoy J, Singer JD, et al. Subcellular localization and cytoplasmic complex status of endogenous Keap1. Genes Cells. 2007 Oct;12(10):1163-78.

93. Itoh K, Wakabayashi N, Katoh Y, Ishii T, O'Connor T, Yamamoto M. Keap1 regulates both cytoplasmic-nuclear shuttling and degradation of Nrf2 in response to electrophiles. Genes Cells. 2003 Apr;8(4):379-91.

94. Zhang M, An C, Gao Y, Leak RK, Chen J, Zhang F. Emerging roles of Nrf2 and phase II antioxidant enzymes in neuroprotection. Prog Neurobiol. 2013 Jan;100:30-47.

95. Hur W, Gray NS. Small molecule modulators of antioxidant response pathway. Curr Opin Chem Biol. 2011 Feb;15(1):162-73.

96. Mitsuishi $\mathrm{Y}$, Motohashi H, Yamamoto M. The Keap1-Nrf2 system in cancers: stress response and anabolic metabolism. Front Oncol. 2012;2:200. 
97. Tong KI, Katoh $\mathrm{Y}$, Kusunoki $\mathrm{H}$, Itoh K, Tanaka T, Yamamoto M. Keap1 recruits Neh2 through binding to ETGE and DLG motifs: characterization of the two-site molecular recognition model. Mol Cell Biol. 2006 Apr;26(8):2887-900.

98. Zhang DD, Lo SC, Sun Z, Habib GM, Lieberman MW, Hannink M. Ubiquitination of Keap1, a BTB-Kelch substrate adaptor protein for Cul3, targets Keap1 for degradation by a proteasome-independent pathway. J Biol Chem. 2005 Aug 26;280(34):30091-9.

99. McMahon M, Thomas N, Itoh K, Yamamoto M, Hayes JD. Redox-regulated turnover of Nrf2 is determined by at least two separate protein domains, the redox-sensitive Neh2 degron and the redox-insensitive Neh6 degron. J Biol Chem. 2004 Jul 23;279(30):31556-67.

100. Pi J, Bai Y, Reece JM, Williams J, Liu D, Freeman ML, et al. Molecular mechanism of human Nrf2 activation and degradation: role of sequential phosphorylation by protein kinase CK2. Free radical biology \& medicine. 2007 Jun 15;42(12):1797-806.

101. Niture SK, Khatri R, Jaiswal AK. Regulation of Nrf2-an update. Free radical biology \& medicine. 2014 Jan;66:36-44.

102. Liu B, Jian Z, Li Q, Li K, Wang Z, Liu L, et al. Baicalein protects human melanocytes from $\mathrm{H}(2) \mathrm{O}(2)$-induced apoptosis via inhibiting mitochondriadependent caspase activation and the p38 MAPK pathway. Free radical biology \& medicine. 2012 Jul 15;53(2):183-93.

103. Brigelius-Flohe R, Flohe L. Basic principles and emerging concepts in the redox control of transcription factors. Antioxidants \& redox signaling. 2011 Oct 15;15(8):2335-81.

104. Nishi T, Shimizu N, Hiramoto M, Sato I, Yamaguchi Y, Hasegawa M, et al. Spatial redox regulation of a critical cysteine residue of NF-kappa B in vivo. J Biol Chem. 2002 Nov 15;277(46):44548-56.

105. Thirlwell C, Schulz L, Dibra H, Beck S. Suffocating cancer: hypoxiaassociated epimutations as targets for cancer therapy. Clin Epigenetics. 2011 Dec 05;3:9.

106. Niecknig H, Tug S, Reyes BD, Kirsch M, Fandrey J, Berchner-Pfannschmidt $\mathrm{U}$. Role of reactive oxygen species in the regulation of HIF-1 by prolyl hydroxylase 2 under mild hypoxia. Free Radic Res. 2012 Jun;46(6):705-17.

107. Martyniuk CJ, Fang B, Koomen JM, Gavin T, Zhang L, Barber DS, et al. Molecular mechanism of glyceraldehyde-3-phosphate dehydrogenase inactivation 
by alpha,beta-unsaturated carbonyl derivatives. Chemical research in toxicology. 2011 Dec 19;24(12):2302-11.

108. Rodriguez CE, Fukuto JM, Taguchi K, Froines J, Cho AK. The interactions of 9,10-phenanthrenequinone with glyceraldehyde-3-phosphate dehydrogenase (GAPDH), a potential site for toxic actions. Chem Biol Interact. 2005 Jun 30;155(12):97-110.

109. Jung DW, Kim WH, Seo S, Oh E, Yim SH, Ha HH, et al. Chemical targeting of GAPDH moonlighting function in cancer cells reveals its role in tubulin regulation. Chem Biol. 2014 Nov 20;21(11):1533-45.

110. Tristan C, Shahani N, Sedlak TW, Sawa A. The diverse functions of GAPDH: views from different subcellular compartments. Cell Signal. 2011 Feb;23(2):317-23. 111. Rahman I. The role of oxidative stress in the pathogenesis of COPD: implications for therapy. Treat Respir Med. 2005;4(3):175-200.

112. Hara MR, Agrawal N, Kim SF, Cascio MB, Fujimuro M, Ozeki Y, et al. Snitrosylated GAPDH initiates apoptotic cell death by nuclear translocation following Siah1 binding. Nat Cell Biol. 2005 Jul;7(7):665-74.

113. Kanwar M, Kowluru RA. Role of glyceraldehyde 3-phosphate dehydrogenase in the development and progression of diabetic retinopathy. Diabetes. 2009 Jan;58(1):227-34.

114. Itakura $M$, Nakajima $H$, Kubo $T$, Semi $Y$, Kume $S$, Higashida $S$, et al. Glyceraldehyde-3-phosphate Dehydrogenase Aggregates Accelerate Amyloid-beta Amyloidogenesis in Alzheimer Disease. J Biol Chem. 2015 Oct 23;290(43):2607287.

115. Galadari S, Rahman A, Pallichankandy S, Thayyullathil F. Reactive oxygen species and cancer paradox: To promote or to suppress? Free radical biology \& medicine. 2017 Mar;104:144-64.

116. Saetta M, Turato G, Maestrelli P, Mapp CE, Fabbri LM. Cellular and structural bases of chronic obstructive pulmonary disease. American journal of respiratory and critical care medicine. 2001 May;163(6):1304-9.

117. Cornwell WD, Kim V, Song C, Rogers TJ. Pathogenesis of inflammation and repair in advanced COPD. Semin Respir Crit Care Med. 2010 Jun;31(3):257-66.

118. Vogelmeier CF, Vestbo J, Hurd SS, Decramer M. Changes in GOLD: today and tomorrow. Lancet Respir Med. 2015 Jun;3(6):424-6. 
119. Sethi S, Murphy TF. Infection in the pathogenesis and course of chronic obstructive pulmonary disease. The New England journal of medicine. $2008 \mathrm{Nov}$ 27;359(22):2355-65.

120. Domej W, Oettl K, Renner W. Oxidative stress and free radicals in COPD-implications and relevance for treatment. International journal of chronic obstructive pulmonary disease. 2014;9:1207-24.

121. Repine JE, Bast A, Lankhorst I. Oxidative stress in chronic obstructive pulmonary disease. Oxidative Stress Study Group. American journal of respiratory and critical care medicine. 1997 Aug;156(2 Pt 1):341-57.

122. Tkaczyk J, Vizek M. Oxidative stress in the lung tissue--sources of reactive oxygen species and antioxidant defence. Prague Med Rep. 2007;108(2):105-14.

123. Kirkham PA, Barnes PJ. Oxidative stress in COPD. Chest. 2013 Jul;144(1):266-73.

124. Jimenez Navarro MF. Comments on the 2016 ESC Guidelines on Cardiovascular Disease Prevention in Clinical Practice. Rev Esp Cardiol (Engl Ed). 2016 Oct;69(10):894-9.

125. Gerhard-Herman MD, Gornik HL, Barrett C, Barshes NR, Corriere MA, Drachman DE, et al. 2016 AHA/ACC Guideline on the Management of Patients With Lower Extremity Peripheral Artery Disease: Executive Summary: A Report of the American College of Cardiology/American Heart Association Task Force on Clinical Practice Guidelines. Circulation. 2016 Nov 13.

126. Dhalla NS, Temsah RM, Netticadan T. Role of oxidative stress in cardiovascular diseases. J Hypertens. 2000 Jun;18(6):655-73.

127. Sudhakar A. History of Cancer, Ancient and Modern Treatment Methods. J Cancer Sci Ther. 2009 Dec 01;1(2):1-4.

128. Hanahan D, Weinberg RA. Hallmarks of cancer: the next generation. Cell. 2011 Mar 04;144(5):646-74.

129. Mattson MP. Hormesis defined. Ageing research reviews. 2008 Jan;7(1):17.

130. Davies KJ. Adaptive homeostasis. Mol Aspects Med. 2016 Jun;49:1-7.

131. Forman HJ, Davies KJ, Ursini F. How do nutritional antioxidants really work: nucleophilic tone and para-hormesis versus free radical scavenging in vivo. Free radical biology \& medicine. 2014 Jan;66:24-35. 
132. Sthijns MM, Weseler AR, Bast A, Haenen GR. Time in Redox Adaptation Processes: From Evolution to Hormesis. International journal of molecular sciences. 2016 Sep 29;17(10). 

Chapter 2

Time in redox adaptation processes: from evolution to hormesis.

Mireille M.J.P.E. Sthijns, Antje R. Weseler, Aalt Bast, Guido R.M.M. Haenen.

International Journal of Molecular Sciences 2016 Sep $29 ; 17(10)$. 


\section{Abstract}

Life on earth has to adapt to the ever changing environment. For example, due to introduction of oxygen in the atmosphere, an antioxidant network evolved to cope with the exposure to oxygen. The adaptive mechanisms of the antioxidant network, specifically the glutathione (GSH) system, are reviewed with a special focus on the time. The quickest adaptive response to oxidative stress is direct enzyme modification, increasing the GSH levels or activating the GSH-dependent protective enzymes. After several hours, a hormetic response is seen at transcriptional level by upregulating Nrf2-mediated expression of enzymes involved in GSH synthesis. In the long run, adaptations occur at the epigenetic and genomic level; for example the ability to synthesize GSH by phototrophic bacteria. Apparently, in an adaptive hormetic response not only the dose, the compound, but also time should be considered. This is essential for targeted interventions aimed to prevent diseases by successfully coping with changes in the environment e.g. oxidative stress.

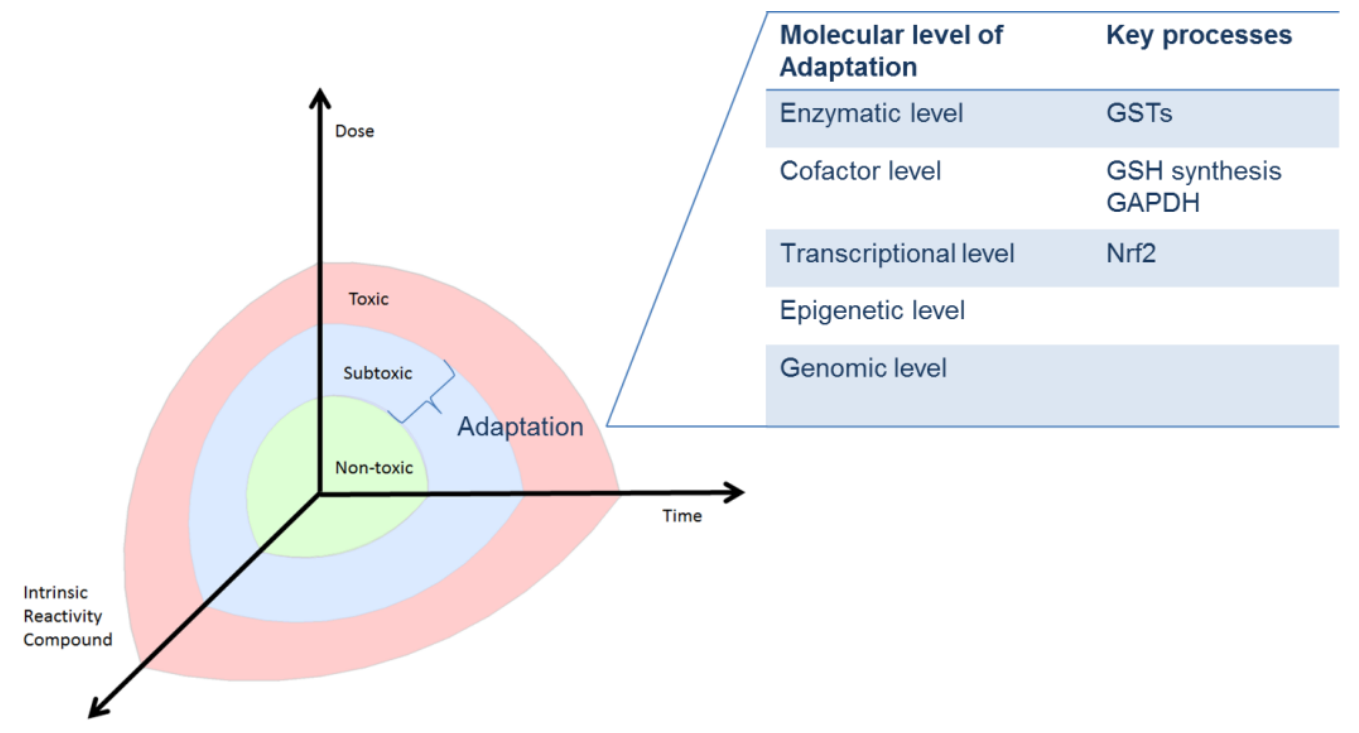




\section{Adaptation or hormesis in general}

The only constant in the atmosphere on earth is that its composition was, is and will never be constant over time. As a consequence, live on earth needs to continuously adapt to an ever changing environment. One of the major changes was the introduction of oxygen $\left(\mathrm{O}_{2}\right)$ in the earth's atmosphere, roughly 2.5 billion years ago, to a level of 21 percent nowadays (1).

The chemical reactivity of oxygen created life forms that acquire their energy from oxidation of compounds. In humans the supply of especially carbohydrates and fats creates a redox potential that fuels all reactions in our body. Eating and exercise will cause the redox potential to gradually fluctuate in time. A sudden block of blood supply drops the oxygen tension and shows that the redox potential can also change drastically within seconds.

If the level of oxygen is too low, then reductive stress is experienced. The low oxygen level results in e.g. an impaired mitochondrial respiratory chain function. Alternatively, when the level of oxygen is too high, oxidative stress will be the result because of an increase in reactive oxygen species (ROS) due to an extensive use of the aerobic mitochondrial respiratory chain. These ROS may, subsequently, lead to cell death and can destroy vital cell structures such as membranes, proteins and DNA. This illustrates the oxygen paradox, which implies that in addition to the dependency of aerobic life on the chemical reactivity of oxygen, the chemical reactivity of oxygen also results in the destruction of vital cell structures. The latter process, known as ageing, will finally kill the organism $(2,3)$.

To mitigate oxygen toxicity and maintain the redox potential within narrow limits, cells are equipped with an intricate antioxidant network. The glutathione (GSH) system is a pivotal part of this network. Apart from antioxidants there are compounds that can induce oxygen toxicity by promoting the formation of ROS.

A peculiar paradox called the oxygen paradox is that adaptation against increased oxygen toxicity is due to oxidative changes in these cells. As will be outlined, this can be due to a low, subtoxic dose of oxidants that can induce changes on a relatively short time scale to antioxidant enzymes (that become more active after oxidation), to transcription factors that act as a redox sensor, and oxidative damage to DNA can lead, by chance, to mutated species that are better equipped to deal with the increased oxygen toxicity i.e. evolution (4).

A response in cells or organisms induced by a low, subtoxic dose of a compound that can induce changes in the environment is called hormesis (5). Next to hormesis, other terms have also been suggested to describe this phenomenon. The historical development of these terms has been thoroughly described by Davies (6). First, Claude Bernard introduced the concept of a constant internal environment in the body in 1865 (7). This 
concept was named "homeostasis". If this internal steady state is triggered by exogenous factors, a state of "heterostasis" may occur as defined by Hans Seyle in the 1970s (8). Later, the term "allostasis" was used instead of "heterostasis". Then, Southem and Ehrlich introduced the current term "hormesis" as an adaptive response inducing either repair mechanisms or enhanced protection after exposure to low doses of a compound (9). This concept is currently still used and Calabrese introduced this concept in toxicological research (10).

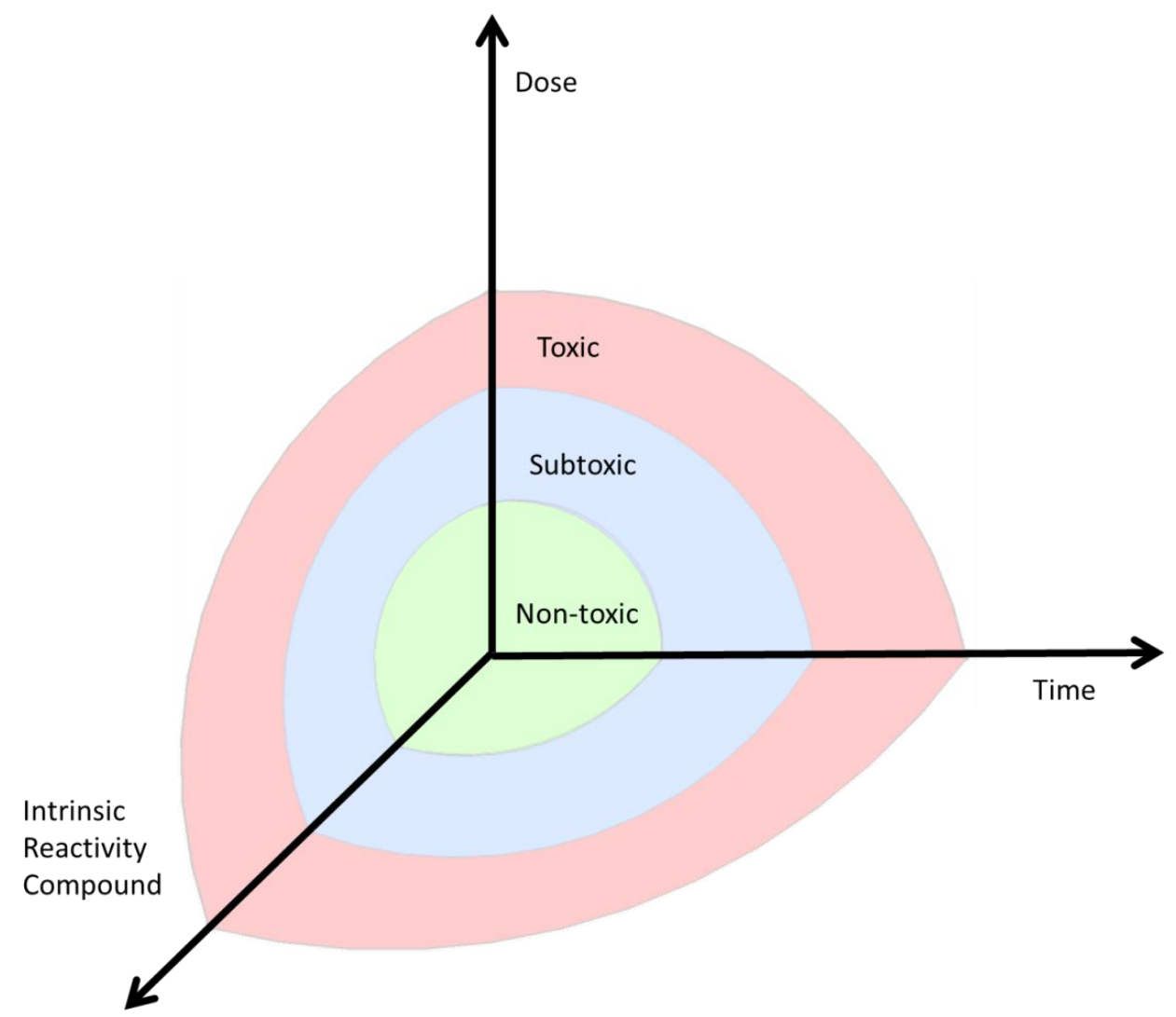

Figure 1: The dose, the compound involved and the timing of exposure influence whether homeostasis exists (green = normal $\neq$ safe, but adapted to toxicity, as low as reasonably achievable (ALARA)), an adaptive response (blue, subtoxic) or toxicity (red, toxic) is induced.

Hormesis is involved in many physiological processes, but in this review we focus on the ability of cells to adapt to a changing redox potential, called redox regulation, which is involved in many different cellular processes. When the changes in redox potential are too drastic, these adaptive systems fail. This will damage organs and 56 
contribute to the development of e.g. cardiovascular diseases, cancer and metabolic diseases. This means that health can be defined as the ability of a cell or organism to keep varying physiological processes within narrow limits (Figure 1). Ideally, formation and scavenging of ROS is well balanced, a condition known as ROS homeostasis. When this balance is disturbed, a specific cellular function can be triggered, for example cell survival. Since these physiological adaptations can lead to a (transient) change or expansion of the internal steady state, Davies recently suggested the term "adaptive homeostasis" (6).

For the present review the ability of the antioxidant network to adapt to the environment will be illustrated by the GSH system with a special focus on the timing of the adaptive response. It should be noted that the adaptive mechanisms are emphasized (hormesis) and not the change in internal steady state (adaptive homeostasis). Relatively short term adaptive mechanisms belong to the phenomenon called hormesis, whereas the development of adaptation on a time scale of months or years are not defined as hormesis, but can be seen as a kind of adaptation.

GSH acts as a reductant that can annihilate the chemical reactivity of ROS in a redox reaction. Various adaptive mechanisms of the GSH system have been developed to cope with the varying oxygen tension.

\subsection{Short term - Enzymatic level}

Sudden changes in the environment calls for a direct adaptation, which should develop within seconds or minutes. First, this can be achieved by modification of enzyme activity of one of the redox defense enzymes of the GSH system. This type of short term adaptation is seen for the (microsomal) glutathione-S-transferases ((M)GSTs) (11). They can protect by catalyzing the scavenging of ROS by GSH. ROS can regulate GST activity. The enzyme contains a redox sensitive sulfhydryl group that readily reacts with ROS. Oxidation of sulfhydryl groups leads to activation. This indicates that the more ROS that are formed, the more sulfhydryl groups are modified, the higher the GST activity becomes, resulting in more protection (12). This is a form of short term redox adaptation by modifying a redoxsensitive group on the enzyme and thereby boosting its activity.

Secondly, short term redox adaptation could also target the synthesis of GSH, by regulating gamma-glutamylcysteine synthetase ( $\gamma \mathrm{GCS})$, the rate-limiting enzyme of GSH synthesis. Г-GCS consists of two subunits, one heavy catalytic subunit ( $\gamma-\mathrm{GCS}-\mathrm{HS})$ of $73 \mathrm{kDa}$ and one light regulatory subunit ( $\gamma$-GCS-LS) of $30 \mathrm{kDa}$. The catalytic subunit has binding sites for the three substrates, $\gamma$-glutamic acid, cysteine and adenosine triphosphate (ATP). The regulatory subunit contains a binding site for GSH and binding of GSH functions as a feedback inhibitor (13). Under normal conditions, $80 \%$ of the available $\gamma \mathrm{GCS}$ is inactive due to the reduction of a disulfide bond interaction between the regulatory and the 
catalytic subunit $(13,14)$. High oxygen tension and excessive ROS production will deplete GSH and restore the disulfide bond. This leads to a conformational change in the catalytic subunit, increasing the affinity of the catalytic subunit for its substrates and thereby stimulating GSH synthesis $(15,16)$ (Table 1$)$. Also via this direct enzymatic redox regulation, a short term adaptation of the protective GSH system can be achieved.

Table 1: The short term adaptive mechanisms. GSH = glutathione; $\mathrm{O}_{2}=$ oxygen; $\gamma \mathrm{GCS}=$ gamma-glutamyl-cysteine synthetase; $\mathrm{GR}=$ glutathione reductase; $\mathrm{NADPH}=$ nicotinamide adenine dinucleotide phosphate.

\begin{tabular}{cc}
\hline Short Term GSH-related Adaptation to $\mathbf{O}_{\mathbf{2}}$ & Target \\
\hline Cofactor & $\mathrm{GSH}$ \\
Enzyme & VGCS \\
Regeneration & GR- > NAPDH \\
\hline
\end{tabular}

Third, adaptation can also be achieved by modification of the supply of enzymatic cofactors to ensure a regeneration of the cellular GSH pool. Scavenging of ROS results in the formation of glutathione disulfide (GSSG). For efficient protection GSSG needs to be converted back into GSH. In order to keep GSH levels high, glutathione reductase (GR) recycles GSH from GSSG at the expense of nicotinamide adenine dinucleotide phosphate (NADPH). To prevent depletion of NADPH an adaptive mechanism has evolved to promote NADPH formation during oxidative stress. During oxidative stress, glyceraldehyde-3phosphate dehydrogenase (GAPDH), an essential enzyme in glycolysis, is inactivated by oxidation. This redirects the energy-generating process into the pentose phosphate pathway (17-20). The switch from reduced nicotinamide adenine dinucleotide (NADH) production by GAPDH to NADPH production by the pentose phosphate pathway could also be seen as a form of short term adaptation. This ensures that GSH levels are sufficient for thiol peroxidases to scavenge $\mathrm{H}_{2} \mathrm{O}_{2}$ and other ROS (17).

It should be noted that previous short term adaptive mechanisms will induce changes that are beneficial on the level of an individual. Enzymatic, but also some transcriptional changes contribute to the survival of a cell, an organism or individual in the ever changing environment.

\subsection{Long term adaptation - transcriptional, epigenetic and genomic level}

In addition to direct short term effects of $\mathrm{O}_{2}$ levels, long term adaptive effects are also induced. For long term adaptive effects a subdivision can be made in effects within hours to days and over generations. 


\subsubsection{Transcriptional level}

First, within hours to days adaptation is mainly induced via altered transcription. For example, ROS induce adaptation by upregulating the endogenous antioxidant level within hours to days. ROS oxidize specific cysteines (Cys273, 288, 151) from Kelch like$\mathrm{ECH}$-associated protein 1 (Keap1), thereby increasing nuclear translocation of nuclear factor (erythroid-derived 2)-like 2 (Nrf2) and enhancing the transcription of antioxidant response element (ARE) containing antioxidant genes including $y G C S$ (21). This subsequently contributes to an increased GSH synthesis and increased protection against oxygen toxicity over a longer term. GSH protects against ischemia-reperfusion damage by maintaining nitric oxide (NO)-induced vasodilation and regulating blood flow to several parts of the body (22). Oxidative stress has been shown to impair NO-induced vasodilation of blood vessels (23). However, $\mathrm{N}$-acetylcysteine (thiol) supplementation improved vasodilation of arteries, indicating GSH protects the vasodilatory function of the vessels (24).

A low $\mathrm{O}_{2}$ tension also induces a long term transcriptionally-mediated adaptive process via the hypoxia-inducible factor 1- $\alpha$ (HIF1 $\alpha$ )-pathway. Prolyl hydroxylases are $\mathrm{O}_{2}$ sensitive. In case of normoxia, prolyl hydrolysases bind $\mathrm{O}_{2}$ to their central ferrous iron moiety and use 2-oxoglutarate as a substrate to hydroxylate HIF1 $\alpha$ on the oxygendependent degradation domain; thereby succinate and carbon dioxide are formed. Then the E3 ligase Von Hippau Lindau protein is able to bind HIF1 $\alpha$ for subsequent ubiquitinylation and proteasomal degradation (25). CBP/p300 coactivator interaction is prevented by hydroxylation of the carboxy-terminal chain of HIF1 $\alpha$ by an oxygendependent asparaginyl hydroxylase (25). However, during hypoxia or low oxygen tension, there is no oxygen present to keep the central iron of prolyl hydroxylase in its ferrous state (25). HIF1 $\alpha$ is not hydroxylated anymore, but contributes to an increase in oxygen tension on a long term. HIF1 $\alpha$ translocates to the nucleus and recruits its coactivators including CBP/P300. Together they bind to the hypoxia response elements and upregulate gene expression of genes including vascular endothelial growth factor (VEGF) or inducible nitric oxide synthase (iNOS) that are involved in angiogenesis or new vessel generation (18). Also on energetic level an adaptive response is induced, promoting anaerobic metabolism by upregulating of gene expression of genes like GAPDH (26).

Moreover, if there is too much oxidative (specifically nitrosative) stress, Ssulphenylated GAPDH is not S-glutathionylated, but S-sulfinated (Figure 2). Instead of a redirection of the metabolic flux to the pentose phosphate pathway, GAPDH does not complex with GAPDH's competitor of Siah protein enhances life (GOSPEL) but with seven in absentia homologs (Siah1), which has nuclear localization signal (18). Nuclear GAPDH is acetylated by the histone acetyltransferase $\mathrm{CBP} / \mathrm{p} 300$. Acetylated GAPDH subsequently stimulates the acetylating function of CBP/p300. Consequently, p300 is activated and is able to activate its downstream targets including the tumor suppressor gene p53 (27). p53 
is known to cause cellular dysfunction and cell death (17). However, this kind of adaptation should be seen on cellular level, indicating that cells that have too much stress are not able to repair the damage induced. For a tissue it could be advantageous that these cells induce their own programmed cell death before they change into dangerous mutated cells.

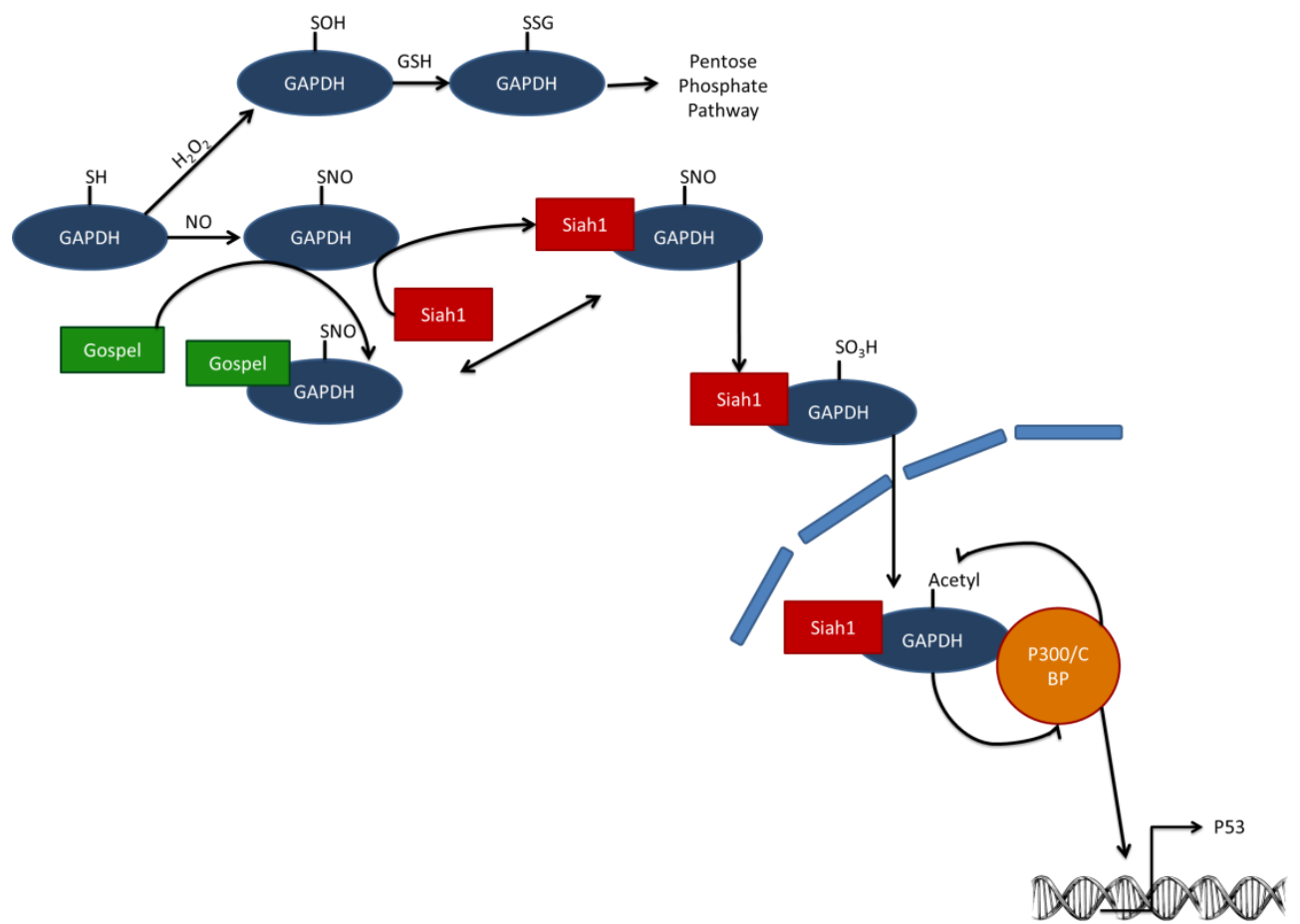

Figure 2: GAPDH can be oxidized and S-glutathionylated, which inhibits its glycolytic function and directs a metabolic switch towards the pentose phosphate pathway. If there is too much stress, not only is GOSPEL $S$-nitrosylated, but also GAPDH, which now prefers binding Siah1 instead of GOSPEL. Siah1 has a nuclear localization signal. In the nucleus, GAPDH complexes with the co-activator CREB-binding protein (CBP)/p300. CBP/p300 subsequently acetylates GAPDH. Acetylated GAPDH boosts the CBP/p300 acetylating function. This enhances transcription of GAPDH downstream target genes including cell death regulator p53. GAPDH = glyceraldehyde-3-phosphate dehydrogenase; GOSPEL = GAPDH's competitor of Siah Protein Enhances Life; Siah1 = Seven in absentia homolog; $\mathrm{GSH}$ = glutathione; $\mathrm{H}_{2} \mathrm{O}_{2}=$ hydrogen peroxide.

\subsubsection{Epigenetic level}

Oxidative stress has been shown to have an effect on epigenetic processes. ROS can result in an oxidation of 5-methylcytosine to 5 -hydroxymethylcytosine $(28,29)$. Also Jumonji C (JmjC) histone lysine demethylases and ten-eleven translocation (TET) DNA 
hydroxylases are regulated by ROS because their ferrous catalytic center is inhibited by oxidation to the ferric state $(28,30,31)$. If the ratio of $\mathrm{NAD}^{+} / \mathrm{NADH}$ is increased, this affects the activity of class III histone deacetylases (HDACS) $(28,30,31)$. An adaptive decrease in HDAC activity is expected. Additionally, $\mathrm{NAD}^{+}$also increases histone deacetylases of the sirtuin family, SIRT1-3 and SIRT6 (32). Due to the low $K_{m}$ values of histone deacetylases for several physiological acyl substrates, $\mathrm{NAD}^{+}$levels control histone deacetylase activity.

The effect of ROS on epigenetic processes could also lead to altered GSH levels. The targeting of histone demethylases like Lsd1 by ROS can induce an epigenetic adaptation of GSH levels. In diabetic retinopathy, Lsd1 inhibition prevented glucose to induce a decrease in $\mathrm{H} 3 \mathrm{~K} 4 \mathrm{me} 1$ at the promotor of the Nrf2-regulated GClC gene, which codes for the catalytic subunit of $\gamma \mathrm{GCS}$ (33). An increase in histone methylation at this site increased GSH synthesis, thereby preventing further development of diabetic retinopathy (33).

Interestingly, also GSH appears to have an effect on regulation of epigenetic processes, for example GSH reduces the activity of DNA methyltransferases (DNMTs) and histone methyltransferases (HMTs) by an inhibition of S-adenosyl methionine synthetase MAT1A which provides the endogenous methyl-donor compound S-adenosyl methionine (SAM) (30). In line with this, addition of N-acetylcysteine seems to decrease global DNA methylation and expression of DNA methyltransferases DNMT1 and DNMT3b (34).

Finally, epigenetic changes can be inherited over generations in humans, indicating that if a mother is exposed to a high level of oxygen that induced oxidative stress and resulted in epigenetic changes thereby up-regulating the GSH system epigenetically, can also be transmitted to the child (35). This is an adaptive mechanism that prepares the child for future exposure and ensures that its ability to survive is high in the future expected environment. Thereby these transgenerational changes in gene expression contribute to the development of species and evolution.

\subsubsection{Genomic level}

Finally, the last long term adaptive response can be found in the genome. Changes in the genome contribute to the development of species and evolution. Only if a species is adapted to the environment, will it survive. The fact that only phototrophic eubacteria contain GSH indicates that GSH evolved in parallel to oxygenic photosynthesis (36). Furthermore, GSTs are evolutionary conserved, and homologous domains can be found back to early prokaryotes. The GSTT class has the most sequence identity with early GSTs, whereas GSTA, GSTM, GSTP are only found after gene duplication in animals and fungi (37). In bacteria and plants, GSTs mainly function as stress-related/stress-resistant proteins (38). However, over time when oxygen appeared in the atmosphere, GSTs evolved from early stress-related/-resistant proteins, to a protection mechanism against 
oxygen toxicity. Today, GSTs function in mammals as part of the phase 2 detoxification system in the liver. They are coupled to cytochrome P450 enzymes that mainly oxidize xenobiotics that are subsequently conjugated with GSH to facilitate excretion by the kidneys by increasing its water solubility (39). However, it should be kept in mind that this metabolism does not always lead to detoxification, but could also be involved in bioactivation (39). All in all, this shows how different parts of the GSH system evolved over time due to the requirements of the changing level of $\mathrm{O}_{2}$ in the environment. Remarkably, changes on the epigenetic and genomic level are more persistent. This means that it is present in an individual and does not easy change over a lifetime. For the individual it is almost not able to induce adaptation on this level, but on the level of species this can be seen as an adaptive response since it ensures the survival of a complete species - the highest outcome of adaptation.

\section{Factors influencing hormesis}

Several factors are involved in inducing an adaptive response and restoring homeostasis. The nature of the induced hormetic response is dependent on the dose, the reactivity of the compound involved and the timing of exposure (Figure 1).

\subsection{Dose and reactivity of the compound and hormesis}

First, the dose of the compound should be high enough to induce a significant adaptive response. However, if the dose is too high, the toxicity of the chemical will overrule and no adaptive response will occur.

Additionally, the compound's reactivity is also crucial, since some compounds are more potent at a lower dose than others in inducing hormesis via a specific pathway. For example auranofin is more potent in inducing adaptation via Nrf2 than sulphoraphane (40). For each compound the borders between non-toxic, sub-toxic and toxic can be different (Figure 1). Additionally also the non-toxic level could be found at another height in the coordinate system, for example for $\mathrm{O}_{2}$ the non-toxic level is defined to be around $21 \%$ of the atmosphere, whereas from acrolein the World Health Organisation recommends to keep the exposure as low as reasonably achievable (ALARA), but at least below the Lowest Observed Adverse Effect Level (LOAEL) of $0.16 \mathrm{mg} / \mathrm{m}^{3}$. Additionally, the effect of the $\alpha, \beta$-unsaturated aldehyde acrolein, a compound released during smoking or formed during incomplete combustion, on hormesis and adaptive responses is determined. 


\section{Adaptation induced by acrolein}

\subsection{Short term - Enzymatic level}

An important detoxification mechanism to protect against the damage of the electrophile acrolein is conjugation with GSH by glutathione-S-transferases (GSTs) in the liver (41-43). GSTs are present in the cytosol, mitochondria and microsomes. In humans, seven different cytosolic GST classes exist. The microsomal GSTs are membrane-bound GSTs and in humans three different isotypes exist that belong to MAPEG (Membrane Associated Proteins in Eicosanoid and Glutathione metabolism) $(42,43)$. GSTs are redox sensitive enzymes that can be modified at their sulfhydryl groups. Xenobiotics like acrolein react with MGST1, a membrane-bound GST, and can increase MGST1 activity both in vitro and in vivo $(12,44)$. This indicates that the more acrolein is present forming adducts with sulfhydryl groups in MGST1, the more the conjugating activity of GST is stimulated, and the more protection is induced (12). This is a form of short term redox adaptation to electrophilic xenobiotics that modify a redox-sensitive enzyme and thereby increase cellular protection against such damaging compounds.

Acrolein also reacts with GSH and induces a short term redox adaptation of the activity of $\gamma G C S$, the rate-limiting enzyme of GSH synthesis. Acrolein stimulates $\gamma$ GCS activity and associated GSH synthesis by two mechanisms. Firstly, it oxidizes the disulfide bond between the regulatory and catalytic subunit of $\gamma \mathrm{GCS}$. This induces a conformational change of the enzyme which increases the affinity of the catalytic subunit for its substrates (15). Secondly, it reduces cellular GSH levels resulting in an enhanced transcription of $\gamma$ GCS' catalytic subunit (16). The last mechanism is defined as long term adaptation and will be explained in further detail in the next paragraph. Eventually, both mechanisms contribute to an enhanced GSH synthesis in cells exposed to acrolein.

\subsection{Long term adaptation}

\subsubsection{Transcriptional level}

As an electrophile, acrolein can also induce adaptation within hours-days by upregulating the transcription of endogenous antioxidants. On a molecular level it was shown that acrolein adducts Keap1, thereby increasing nuclear translocation of Nrf2 and enhancing transcription of ARE containing antioxidant genes including $\gamma \mathrm{GCS}$ (45). This subsequently contributes to an increased GSH synthesis and increased protection against these damaging electrophiles on a longer term.

\subsubsection{Epigenetic level}

In addition, acrolein has been shown to decrease the activity of HDACs, because it adducts thiols in this enzyme. Since HDACs including sirtuins are inhibited, lysine residues on histones are not deacetylated anymore and this prevents HDACs from 
counteracting expression of specific genes maybe including the rate-limiting enzyme of GSH synthesis, $\gamma \mathrm{GCS}$ (28). In the end this will result in an upregulation of $\gamma \mathrm{GCS}$ (28). In the end this will result in an up-regulation of $\gamma \mathrm{GCS}$. Additionally, acrolein is thought to activate p38MAPK that have been shown to have an effect on the activity and expression of certain lysine acetyl-transferases (e.g.KAT3A/KAT3B(CBP/p300)) $(36,46)$. Also via this way, the expression of GSH system-related genes could be modified.

\section{Adaptation induced by one compound protects against another}

Additionally, some compounds can induce an adaptation that provides protection against the toxic effects of another compound (47). This phenomenon is called crosshormesis. This phenomenon implies that exposure to a non-toxic dose of a specific compound induces protection against another compound. For example hydrogen peroxide pre-treatment with a low dose has been shown to protect against acetate, ethanol and propionate (48). If the pretreatment is done with a compound that is generally defined as healthy, then crosshormesis is referred to as para-hormesis (49). For example, relatively nontoxic nutritional antioxidants like flavonoids can upregulate the endogenous antioxidant response during oxidative stress thereby protecting against the damaging effects of electrophiles (50). From a toxicological point of view, both processes are the same, because Paracelsus's principle indicates that if the dose is high enough any compound can be toxic.

\section{Adaptation induced by dietary antioxidants}

Additionally, next to the societal interpretation of flavonoids being healthy, these flavonoids compared to $\mathrm{O}_{2}$ and acrolein also show another level of hormesis induction, which makes it very interesting to further examine the hormetic mechanism indirectly induced by flavonoids. Next to the finding that other compounds can induce a similar mechanism of hormesis, the flavonoid is on its own does not induce hormesis. However, if the cell experiences an increased level of oxidative stress, a flavonoid functions as a direct antioxidant, scavenging any damaging ROS (Figure 3). During this scavenging flavonoids are oxidized forming a flavonoid quinone that selectively channels the reactivity in the endogenous antioxidant system by reacting with thiols like GSH. If the level of stress increases even more, also thiol-containing proteins are targeted, with regard to flavonoids, this could be Keap1, as mentioned earlier in this article (50). This results in increased expression of Nrf2-mediated antioxidant genes and increases protection. However, if the level of stress is even higher and time of exposure is increased, even more thiol-containing 
proteins are attacked like $\mathrm{Ca}^{2+}$-ATPase and GAPDH, this could selectively induce toxicity by inducing apoptosis $(51,52)$.

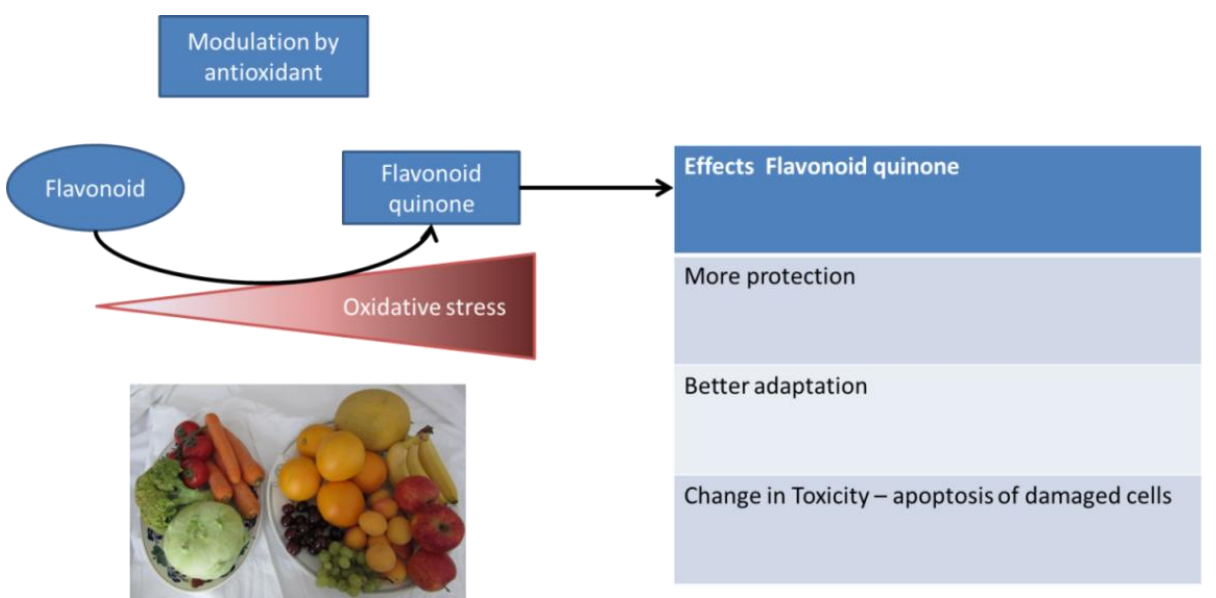

Figure 3: Example of exposure with a flavonoid that only induces different levels of redox adaptation in time in its oxidized form (flavonoid quinone). The more oxidative stress is present, the more flavonoid scavenges the present ROS and the more flavonoid quinone is formed.

\section{Effects of hormesis - remodeling of the GSH system}

Adaptation processes in time have physiological consequences. For example, in the epithelial lining fluid of chronic smokers, who are regularly exposed to acrolein, the GSH levels are twofold increased compared to non smokers or acute smokers (13). This suggests that inhaled cigarette smoke compounds probably induce an adaptive response of the protecting endogenous antioxidant system on a long term. Additionally, current smokers and ex-smokers had an increased DNA methylation level at the promotor of $\gamma \mathrm{GCS}$. This epigenetic modification probably contributes to the increased level of GSH in the epithelial lining fluid of these smokers (53).

Additionally, multiple studies show that athletes who experience a burst in oxidative stress during training have a higher antioxidant capacity than their sedentary controls, independently of their antioxidant intake (54). Antioxidant capacity was determined as trolox-equivalent antioxidant capacity (TEAC) and activity of the antioxidant enzymes superoxide dismutase, glutathione peroxidase, and glutathione reductase was measured in addition to $\alpha$-tocopherol $(54,55)$. Except for $\alpha$-tocopherol, all plasma concentrations of antioxidant parameters in athletes were above resting values and remained high for a longer time after exercise compared to sedentary subjects (55). Furthermore, oxidative stress markers were measured including malondialdehyde representing the degree of lipid peroxidation (55). Exercise is accompanied with an increase in oxidative stress, which 
finally results in adaptive changes in the (endogenous) antioxidant levels. Additionally, in endurance runners a negative association between total antioxidant status and protein carbonyl content, measuring oxidative damage towards proteins, was seen (56). Moreover, when comparing the malondialdehyde and protein carbonyl levels in untrained, aerobically trained, and anaerobically trained individuals before and after exercise, remarkably only in untrained individuals the malondialdehyde and protein carbonyl level increased, whereas total antioxidant levels did not change (57). Furthermore, depending on the type-endurance or resistance exercise-exercise also induces adaptive changes in muscle fiber types, causes muscle hypertrophy, induces metabolic adaptation by increasing size and number of mitochondria, and changes the activity of oxidative enzymes. These adaptations have been shown to result in long term cardiac and respiratory changes by increasing cardiac output and stroke volume and stimulation of capillary formation. This, and the higher pulmonary blood flow, keep blood pressure stable even when changing the exercise level by increasing tidal volume, respiratory rate, and pulmonary diffusion (58). Apart from the centrally-found physiological changes, also on the organ level (for example in the central nervous system and in (skeletal) muscles), physiological indications of redox-related adaptive responses could be found after exercise-based regimes (59-62). When comparing the antioxidant levels, protein carbonyl content, and lipid peroxidation degradation products in the brain cortex and hippocampus of exercise- and non-exercise-exposed female CD1 mice it was found that only in exercise-exposed mice the antioxidant levels increased over time, whereas the protein carbonyl content and lipid peroxidation degradation products decreased $(59,60)$. Regular exercise or training has been shown to induce ROS-mediated adaptive responses in the skeletal muscles, but also in the heart and, finally, result in increased expression and level of endogenous antioxidant enzymes (61). Remarkably, in the different organs the exercise-induced adaptive response seems rather similar, despite the fact that skeletal muscle, liver, and brain have different metabolic rates and functions during exercise (62). The overall effect of exercise in the different organs is less oxidative damage, an increased level of endogenous antioxidant systems, repair processes, and a better protection against a future challenge with oxidative stress.

Next to exercise, caloric restriction has also shown to have physiological effects due to adaptation. The harsher the caloric restriction in rats, the higher the level of malondialdehyde and the lower the level of glutathione and antioxidant enzymes, including superoxide dismutase (63). This implicates that fasting or caloric restriction apparently triggers crucial hormetic improvements in the redox biology of cells and tissues.

Moreover, another example of the physiological consequence of adaptation is the effect of radiation on the human body. For a long time the linear no threshold model was applied, indicating that the more a subject is exposed to radiation, the higher the risk 66 
becomes to develop cancer. However, recently more evidence has been found to support a phenomenon called radiation hormesis. Repeated low dose radiation appears to induce adaptive mechanisms that result in protection and reduce the risk to develop cancer (64). A meta-analysis of 18 studies performed between 1986 and 2012 showed that in mice doses between $0-100$ mGy results in protection whereas above 100 mGy the probability to induce damage is higher than the possibility of damage reduction (65). Furthermore, in medical professionals working with radiation before 1950 a high death rate due to leukemia was seen, while after 1950 when the maximal exposure limit was reduced from $30,000 \mathrm{mSv} /$ year to $50 \mathrm{mSv} /$ year, medical professionals working with radiation have lower mortality rates than other medical specialists not working with radiation (66). Finally, also in Chernobyl birds, at first GSH levels decreased and oxidative damage was increased, but over time depending on the level of background radiation, oxidative stress and DNA damage showed a reduction, while an increase in GSH levels and body condition was observed, indicating that over time organisms can adapt to their exposure (67). The mechanisms by which radiation induces adaptation, are largely unknown, but certain findings underline that the immune system is boosted by low dose radiation, DNA repair is enhanced and endogenous antioxidants are increased, probably by the activation of the Nrf2 pathway (66).

\section{Timing and hormesis}

Finally, timing is very essential for hormesis to take place. The timing between the first and second exposure is critical in the fact that if these exposures follow each other too rapidly, there is not enough time for the induction of a protective adaptive response and the second treatment might be lethal. Moreover, if the time between these exposures is too long, the adaptive effect will fade away and no effect of the first treatment will be seen. The timing between two exposures is also compound-dependent. Furthermore, it would be very interesting to elucidate the dynamics of this adaptation process. Could cells or organisms exposed repeatedly to a low dose of a compound continuously lead to a persistent adaptive response or will the effect of this fade out, causing a kind of desensitization? Additionally, the duration of the adaptive effect is another factor involved in adaptation (Figure 4). We distinguish short term and long term adaptation. Short term adaptation can be defined as a direct adaptation carried out in a few seconds or minutes for example an enzymatic regulation. Long term adaptation includes an adaptation from which the effects can even be noticed after hours or days (transcriptional regulation), months or years or even generations (epigenetic and genomic effects). 


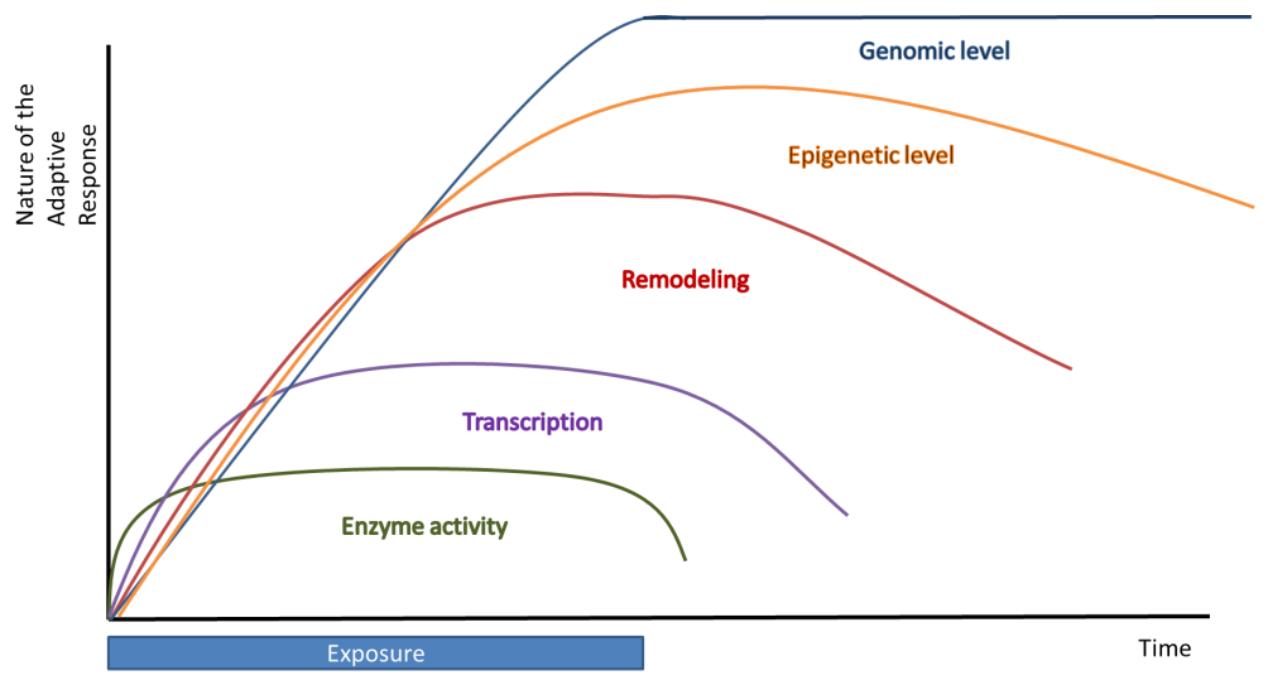

Figure 4: Multiple levels of redox adaptation over time.

\section{Conclusion}

In conclusion, changes in the environment will induce adaptive mechanisms that mitigate or eventually counteract the change. For example, adaptation ensures that an increase in oxygen pressure is not lethal by upgrading of the antioxidant defense (Figure 1). Chronic changes of the environment may lead to a new steady state. As illustrated in Table 2 the longer the exposure time, the more persistent the adaptation becomes or the organism can't cope with the exposure any longer and dies. The most drastic fatal adaptation is seen at genome level, which may even lead to new species. In contrast, adaptation at the functional protein level is characterized by more direct, temporary and subtle changes (Table 2). Thus, multiple levels of redox adaptation exist in time, which differ in time of onset and duration.

Short term adaptation includes direct enzymatic modification, or a change in the available amount of cofactors, or an alteration of signaling molecules in a specific pathway which will result in an effect in seconds or minutes. Redox regulation on a transcriptional level generally takes hours or days. These relatively short term adaptive mechanisms belong to the phenomenon called hormesis. In addition, adaptation can develop on a time scale of months or years. These are not covered by the term hormesis, but can be seen as a kind of adaptation. This includes remodeling of the GSH system, and epigenetic and genomic effects. Remodeling could lead to beneficial (increase in antioxidant capacity after exercise) or deleterious (apoptosis) consequences and result in another phenotype. The epigenetic changes have more persistent adaptive effects that may last over generations. The most persistent and drastic form of adaptation is seen when the genome 68 
is adjusted. In the current paradigm a random mutation of DNA may lead to species that better fit in an environment that has changed.

Table 2: The effect of time and frequency of exposure on adaptive changes. If the environment changes (for example the $\mathrm{O}_{2}$ pressure), the steady state will be restored directly, in the long term the steady state will be adapted.

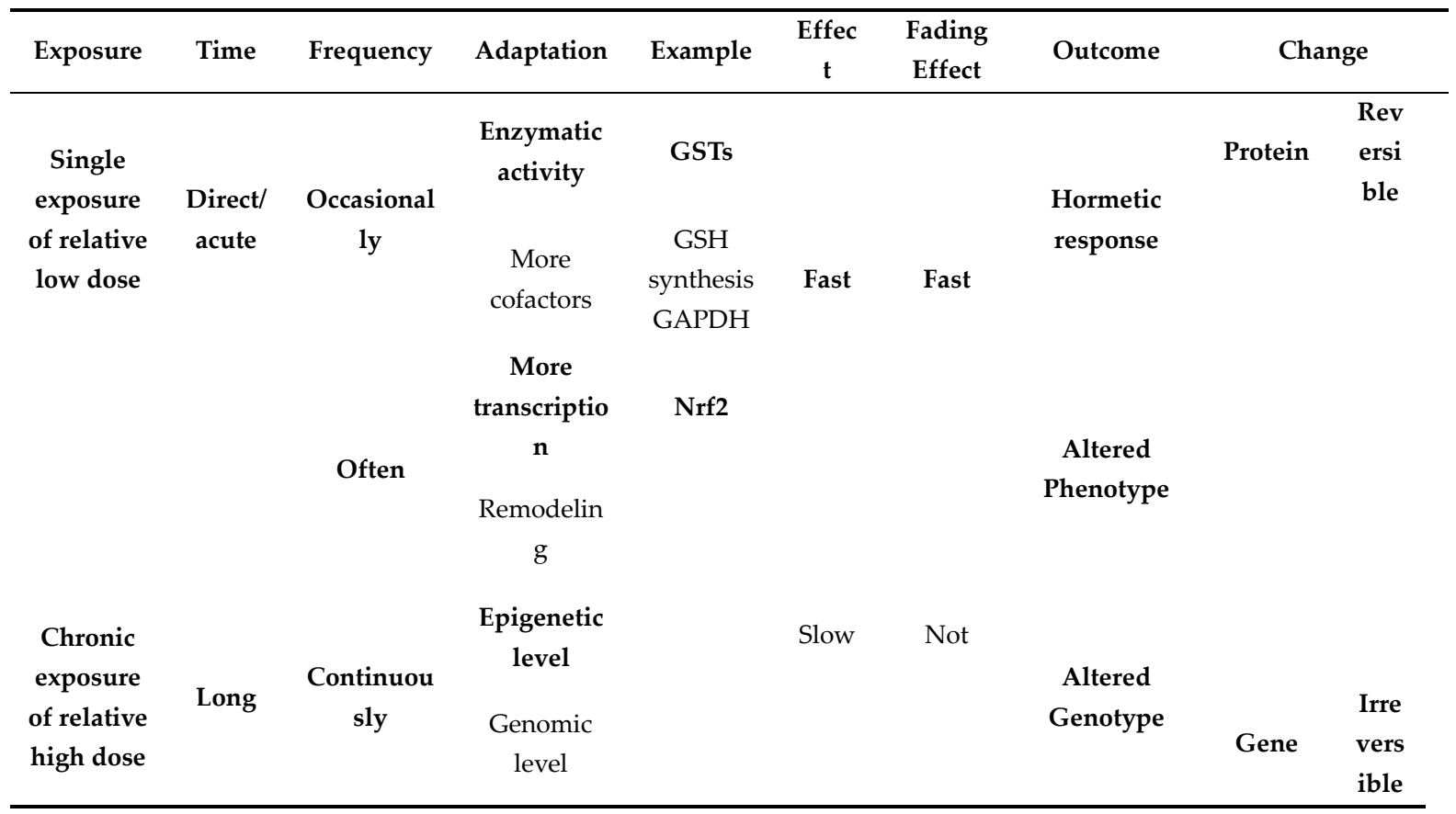

It should be realized that ultimately all forms of adaptation are encoded in the genome are universal among living organisms, including the presence of redox sensitive thiol groups in the microsomal GST and Keap1. Based on the aforementioned paradigm that in the genome only information that fits is preserved, it might be reasoned that the ability of an organism to give a hormetic response is beneficial on an evolutionary level. Despite the energy the cell has to invest in a hormetic response, this, as also discussed in this review, might come too early or too late and might also be unnecessary because the change in the environment was only temporary. This might be a problem for an individual (because in those cases it will not survive), but in the long term those "investments" (of individuals) are vital/crucial for life on earth to survive and evolve, and in this way adapt to the ever changing environment. 
In summary, many physiological processes follow a hormetic response and several factors are involved in inducing an adaptive response. When investigating a biological response, adaptive mechanisms should be taken into account considering not only the dose, the compound, but also time. Advanced knowledge about the hormetic responses will open new avenues to promote disease interventions aimed at increasing our ability to adapt. Increasing our ability to adapt can be used to cope with changes in the environment like oxidative stress that otherwise would result in diseases like cardiovascular disease, to cancer and metabolic diseases. 


\section{References}

1. Lyons TW, Reinhard CT, Planavsky NJ. The rise of oxygen in Earth's early ocean and atmosphere. Nature. 2014 Feb 20;506(7488):307-15.

2. Davies KJ. Oxidative stress: the paradox of aerobic life. Biochem Soc Symp. 1995;61:1-31.

3. Bast A, Haenen GR, Doelman CJ. Oxidants and antioxidants: state of the art. Am J Med. 1991 Sep 30;91(3C):2S-13S.

4. Trachootham D, Lu W, Ogasawara MA, Nilsa RD, Huang P. Redox regulation of cell survival. Antioxidants \& redox signaling. 2008 Aug;10(8):1343-74. 5. Mattson MP. Hormesis defined. Ageing research reviews. 2008 Jan;7(1):17.

6. Davies KJ. Adaptive homeostasis. Mol Aspects Med. 2016 Jun;49:1-7.

7. Bernard C. An introduction to the study of experimental medicine (originally published in 1865, Dover ed. Macmillan \& Co., Ltd. 1927, first English, 1957). 1865.

8. Selye H. Homeostasis and heterostasis. Perspect Biol Med. 1973 Spring;16(3):441-5.

9. Southem CM, Ehrlich J. Effects of extract of western red-cedar heartwood on certain wood-decaying fungi in culture. Phytopathology. 1943;33:517-24.

10. Calabrese EJ. Hormesis: a revolution in toxicology, risk assessment and medicine. EMBO Rep. 2004 Oct;5 Spec No:S37-40.

11. Hayes JD, Pulford DJ. The glutathione S-transferase supergene family: regulation of GST and the contribution of the isoenzymes to cancer chemoprotection and drug resistance. Crit Rev Biochem Mol Biol. 1995;30(6):445600.

12. Haenen GR, Jansen FP, Vermeulen NP, Bast A. Activation of the microsomal glutathione S-transferase by metabolites of alpha-methyldopa. Archives of biochemistry and biophysics. 1991 May 15;287(1):48-52.

13. Rahman I, MacNee W. Lung glutathione and oxidative stress: implications in cigarette smoke-induced airway disease. The American journal of physiology. 1999 Dec;277(6 Pt 1):L1067-88.

14. Rahman I, Bel A, Mulier B, Donaldson K, MacNee W. Differential regulation of glutathione by oxidants and dexamethasone in alveolar epithelial cells. Am J Physiol. 1998 Jul;275(1 Pt 1):L80-6. 
15. Huang CS, Chang LS, Anderson ME, Meister A. Catalytic and regulatory properties of the heavy subunit of rat kidney gamma-glutamylcysteine synthetase. J Biol Chem. 1993 Sep 15;268(26):19675-80.

16. Tian L, Shi MM, Forman HJ. Increased transcription of the regulatory subunit of gamma-glutamylcysteine synthetase in rat lung epithelial L2 cells exposed to oxidative stress or glutathione depletion. Arch Biochem Biophys. 1997 Jun 1;342(1):126-33.

17. Peralta D, Bronowska AK, Morgan B, Doka E, Van Laer K, Nagy P, et al. A proton relay enhances $\mathrm{H} 2 \mathrm{O} 2$ sensitivity of GAPDH to facilitate metabolic adaptation. Nat Chem Biol. 2015 Feb;11(2):156-63.

18. Tristan C, Shahani N, Sedlak TW, Sawa A. The diverse functions of GAPDH: views from different subcellular compartments. Cell Signal. 2011 Feb;23(2):317-23. 19. Hildebrandt T, Knuesting J, Berndt C, Morgan B, Scheibe R. Cytosolic thiol switches regulating basic cellular functions: GAPDH as an information hub? Biol Chem. 2015 May;396(5):523-37.

20. Kuehne A, Emmert $H$, Soehle J, Winnefeld M, Fischer F, Wenck $H$, et al. Acute Activation of Oxidative Pentose Phosphate Pathway as First-Line Response to Oxidative Stress in Human Skin Cells. Mol Cell. 2015 Aug 6;59(3):359-71.

21. Zhang J, Wang $X$, Vikash $V$, Ye $Q$, Wu D, Liu $Y$, et al. ROS and ROSMediated Cellular Signaling. Oxid Med Cell Longev. 2016;2016:4350965.

22. Cheung PY, Wang W, Schulz R. Glutathione protects against myocardial ischemia-reperfusion injury by detoxifying peroxynitrite. J Mol Cell Cardiol. 2000 Sep;32(9):1669-78.

23. Thengchaisri N, Hein TW, Wang W, Xu X, Li Z, Fossum TW, et al. Upregulation of arginase by $\mathrm{H} 2 \mathrm{O} 2$ impairs endothelium-dependent nitric oxidemediated dilation of coronary arterioles. Arteriosclerosis, thrombosis, and vascular biology. 2006 Sep;26(9):2035-42.

24. Andrews NP, Prasad A, Quyyumi AA. N-acetylcysteine improves coronary and peripheral vascular function. Journal of the American College of Cardiology. 2001 Jan;37(1):117-23.

25. Wenger RH. Cellular adaptation to hypoxia: O2-sensing protein hydroxylases, hypoxia-inducible transcription factors, and O2-regulated gene expression. FASEB journal : official publication of the Federation of American Societies for Experimental Biology. 2002 Aug;16(10):1151-62. 
26. Zhang JY, Zhang F, Hong CQ, Giuliano AE, Cui XJ, Zhou GJ, et al. Critical protein GAPDH and its regulatory mechanisms in cancer cells. Cancer biology \& medicine. 2015 Mar;12(1):10-22.

27. Touyz RM, Briones AM. Reactive oxygen species and vascular biology: implications in human hypertension. Hypertens Res. 2011 Jan;34(1):5-14.

28. Mikhed Y, Gorlach A, Knaus UG, Daiber A. Redox regulation of genome stability by effects on gene expression, epigenetic pathways and DNA damage/repair. Redox biology. 2015 Aug;5:275-89.

29. Crawford DJ, Liu MY, Nabel CS, Cao XJ, Garcia BA, Kohli RM. Tet2 Catalyzes Stepwise 5-Methylcytosine Oxidation by an Iterative and de novo Mechanism. J Am Chem Soc. 2016 Jan 27;138(3):730-3.

30. Garcia-Gimenez JL, Ibanez-Cabellos JS, Seco-Cervera M, Pallardo FV. Glutathione and cellular redox control in epigenetic regulation. Free radical biology \& medicine. 2014 Oct;75 Suppl 1:S3.

31. Niu Y, DesMarais TL, Tong Z, Yao Y, Costa M. Oxidative stress alters global histone modification and DNA methylation. Free radical biology \& medicine. 2015 May;82:22-8.

32. Feldman JL, Dittenhafer-Reed KE, Kudo N, Thelen JN, Ito A, Yoshida M, et al. Kinetic and Structural Basis for Acyl-Group Selectivity and NAD(+) Dependence in Sirtuin-Catalyzed Deacylation. Biochemistry. 2015 May 19;54(19):3037-50.

33. Mishra $M$, Zhong $Q$, Kowluru RA. Epigenetic modifications of Nrf2mediated glutamate-cysteine ligase: implications for the development of diabetic retinopathy and the metabolic memory phenomenon associated with its continued progression. Free radical biology \& medicine. 2014 Oct;75:129-39.

34. Campos AC, Molognoni F, Melo FH, Galdieri LC, Carneiro CR, D'Almeida V, et al. Oxidative stress modulates DNA methylation during melanocyte anchorage blockade associated with malignant transformation. Neoplasia. 2007 Dec;9(12):1111-21.

35. Sen A, Heredia N, Senut MC, Land S, Hollocher $K$, Lu X, et al. Multigenerational epigenetic inheritance in humans: DNA methylation changes associated with maternal exposure to lead can be transmitted to the grandchildren. Sci Rep. 2015;5:14466.

36. Fahey RC, Buschbacher RM, Newton GL. The evolution of glutathione metabolism in phototrophic microorganisms. J Mol Evol. 1987;25:81-8. 
37. Pemble SE, Taylor JB. An evolutionary perspective on glutathione transferases inferred from class-theta glutathione transferase cDNA sequences. Biochem J. 1992 Nov 1;287 ( Pt 3):957-63.

38. Sheehan D, Meade G, Foley VM, Dowd CA. Structure, function and evolution of glutathione transferases: implications for classification of nonmammalian members of an ancient enzyme superfamily. Biochem J. $2001 \mathrm{Nov}$ 15;360(Pt 1):1-16.

39. Bast A, Haenen GR. Cytochrome P-450 and glutathione: what is the significance of their interrelationship in lipid peroxidation? TIBS. 1984.

40. Johansson K, Cebula M, Rengby O, Dreij K, Carlstrom KE, Sigmundsson K, et al. Cross Talk in HEK293 Cells Between Nrf2, HIF, and NF-kappaB Activities upon Challenges with Redox Therapeutics Characterized with Single-Cell Resolution. Antioxidants \& redox signaling. 2015 Nov 11.

41. Siritantikorn A, Johansson K, Ahlen K, Rinaldi R, Suthiphongchai T, Wilairat $P$, et al. Protection of cells from oxidative stress by microsomal glutathione transferase 1. Biochemical and biophysical research communications. $2007 \mathrm{Apr}$ 6;355(2):592-6.

42. Johansson K, Jarvliden J, Gogvadze V, Morgenstern R. Multiple roles of microsomal glutathione transferase 1 in cellular protection: a mechanistic study. Free radical biology \& medicine. 2010 Dec 1;49(11):1638-45.

43. Morgenstern R, Zhang J, Johansson K. Microsomal glutathione transferase 1: mechanism and functional roles. Drug metabolism reviews. 2011 May;43(2):300-6.

44. Haenen GR, Vermeulen NP, Tai Tin Tsoi JN, Ragetli HM, Timmerman H, Blast A. Activation of the microsomal glutathione-S-transferase and reduction of the glutathione dependent protection against lipid peroxidation by acrolein. Biochemical pharmacology. 1988 May 15;37(10):1933-8.

45. Sthijns MM, Randall MJ, Bast A, Haenen GR. Adaptation to acrolein through upregulating the protection by glutathione in human bronchial epithelial cells: the materialization of the hormesis concept. Biochemical and biophysical research communications. 2014 Apr 18;446(4):1029-34.

46. Tsai YJ, Tsai T, Peng PC, Li PT, Chen CT. Histone acetyltransferase p300 is induced by P38MAPK after photodynamic therapy: the therapeutic response is increased by the p300HAT inhibitor anacardic acid. Free radical biology \& medicine. 2015 Sep;86:118-32. 
47. Zhang $\mathrm{Q}$, Pi J, Woods CG, Andersen ME. Phase I to II cross-induction of xenobiotic metabolizing enzymes: a feedforward control mechanism for potential hormetic responses. Toxicology and applied pharmacology. 2009 Jun 15;237(3):345-56.

48. Semchyshyn HM. Hormetic concentrations of hydrogen peroxide but not ethanol induce cross-adaptation to different stresses in budding yeast. Int J Microbiol. 2014;2014:485792.

49. Forman HJ, Davies KJ, Ursini F. How do nutritional antioxidants really work: nucleophilic tone and para-hormesis versus free radical scavenging in vivo. Free radical biology \& medicine. 2014 Jan;66:24-35.

50. Lemmens KJA, Sthijns MMJPE, van der Vijgh WJF, Bast A, Haenen GRMM. The antioxidant flavonoid monoHER provides efficient protection and induces the innate Nrf2 mediated adaptation in endothelial cells subjected to oxidative stress. PharmaNutrition. 2014;2(3):69-74.

51. Boots AW, Li H, Schins RP, Duffin R, Heemskerk JW, Bast A, et al. The quercetin paradox. Toxicology and applied pharmacology. 2007 Jul 1;222(1):89-96. 52. Boots AW, Haenen GR, den Hartog GJ, Bast A. Oxidative damage shifts from lipid peroxidation to thiol arylation by catechol-containing antioxidants. Biochimica et biophysica acta. 2002 Aug 8;1583(3):279-84.

53. Cheng L, Liu J, Li B, Liu S, Li X, Tu H. Cigarette Smoke-Induced Hypermethylation of the GCLC Gene Is Associated With COPD. Chest. 2016 Feb;149(2):474-82.

54. Carlsohn A, Rohn S, Bittmann F, Raila J, Mayer F, Schweigert FJ. Exercise increases the plasma antioxidant capacity of adolescent athletes. Ann Nutr Metab. 2008;53(2):96-103.

55. El Abed K, Rebai H, Bloomer RJ, Trabelsi K, Masmoudi L, Zbidi A, et al. Antioxidant status and oxidative stress at rest and in response to acute exercise in judokas and sedentary men. J Strength Cond Res. 2011 Sep;25(9):2400-9.

56. Falone S, Mirabilio A, Passerini A, Izzicupo P, Cacchio M, Gallina S, et al. Aerobic performance and antioxidant protection in runners. Int J Sports Med. 2009 Nov;30(11):782-8.

57. Park SY, Kwak YS. Impact of aerobic and anaerobic exercise training on oxidative stress and antioxidant defense in athletes. J Exerc Rehabil. 2016 Apr;12(2):113-7.

58. Manley AF. Physical Activity and Health: DIANE Publishing; 1996. 
59. Falone S, D'Alessandro A, Mirabilio A, Cacchio M, Di llio C, Di Loreto S, et al. Late-onset running biphasically improves redox balance, energy- and methylglyoxal-related status, as well as SIRT1 expression in mouse hippocampus. PLoS One. 2012;7(10):e48334.

60. Falone S, D'Alessandro A, Mirabilio A, Petruccelli G, Cacchio M, Di Ilio C, et al. Long term running biphasically improves methylglyoxal-related metabolism, redox homeostasis and neurotrophic support within adult mouse brain cortex. PLoS One. 2012;7(2):e31401.

61. Powers SK, Radak Z, Ji LL. Exercise-induced oxidative stress: past, present and future. J Physiol. 2016 Feb 19.

62. Radak Z, Chung HY, Goto S. Systemic adaptation to oxidative challenge induced by regular exercise. Free radical biology \& medicine. 2008 Jan 15;44(2):153-9.

63. Stankovic M, Mladenovic D, Ninkovic M, Vucevic D, Tomasevic T, Radosavljevic T. Effects of caloric restriction on oxidative stress parameters. Gen Physiol Biophys. 2013 Jun;32(2):277-83.

64. Vaiserman AM. Radiation hormesis: historical perspective and implications for low-dose cancer risk assessment. Dose Response. 2010;8(2):17291.

65. Feinendegen LE. Quantification of Adaptive Protection Following Lowdose Irradiation. Health Phys. 2016 Mar;110(3):276-80.

66. Boots AW, Balk JM, Bast A, Haenen GR. The reversibility of the glutathionyl-quercetin adduct spreads oxidized quercetin-induced toxicity. Biochemical and biophysical research communications. 2005 Dec 16;338(2):923-9. 67. Galvan I, Bonisoli-Alquati A, Jenkinson S, Ghanem G, Wakamatsu K, Mousseau TA, et al. Chronic exposure to low-dose radiation at Chernobyl favours adaptation to oxidative stress in birds. Functional Ecology. 2014;28:1387-403. 


Chapter 3

Adaptation to acrolein through upregulating the protection by glutathione in human bronchial epithelial cells: the materialization of the hormesis concept.

Mireille M.J.P.E. Sthijns, Matthew J. Randall, Aalt Bast, Guido R.M.M. Haenen.

Biochemical and Biophysical Research Communications. 2014 Apr 18;446(4):1029-34. 


\section{Abstract}

Acrolein is a thiol reactive compound present in cigarette smoke and plays a pivotal role in the deleterious effects of smoking. Acrolein causes toxicity in human bronchial epithelial cells in a dose dependent manner. GSH forms the first line of defense against acrolein-induced toxicity. At high doses of acrolein $(\geq 10 \mu \mathrm{M})$ the capacity of the cellular protection by GSH is overwhelmed and GSH is not able to quench all the acrolein, resulting in cytotoxicity.

At a relatively low dose of acrolein $(3 \mu \mathrm{M})$, no cytotoxicity is observed due to protection by GSH. Moreover we found that exposure to a low dose of acrolein protects cells against the toxic effect of a second higher dose of acrolein. The adaptation to acrolein is induced via Nrf2 mediated gene expression of $\gamma$-glutamylcysteine synthetase leading to elevated GSH levels. This upregulation of the protection by GSH demonstrates a hormetic response to acrolein.

Hormesis is an adaptive or compensatory response induced by a relatively subtle challenge of homeostasis by a toxic compound. Insight into the mechanism of hormesis is mandatory for a more accurate societal regulation of toxic compounds.

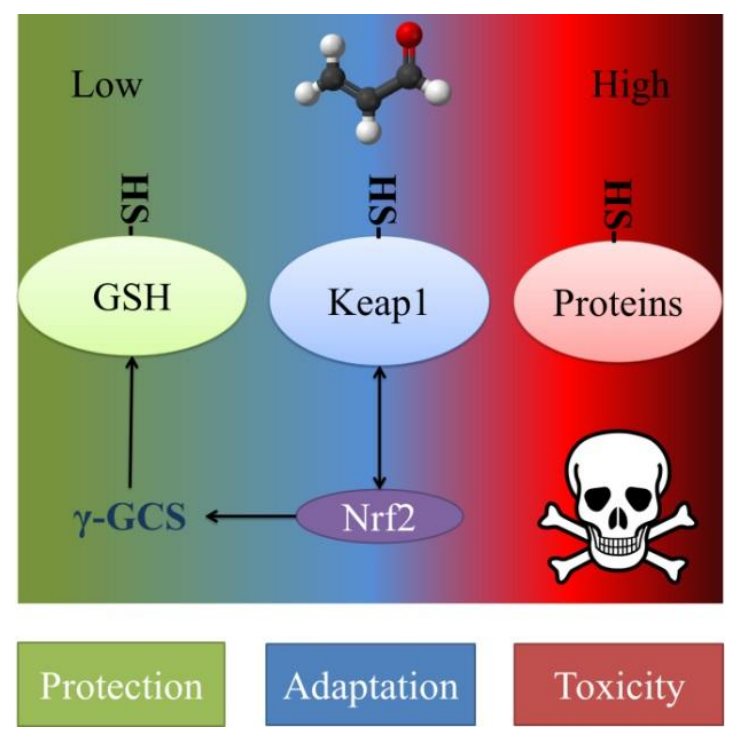




\section{Introduction}

The human body is continuously exposed to a wide variety of compounds present in the environment that can have toxic effects at a relatively low dose, such as polycyclic aromatic hydrocarbons, aldehydes and metals (1). These exogenous compounds can be inhaled by breathing polluted air, ingested by consuming food or contaminated water or absorbed via the skin. A relatively high dose of an exogenous compound results in damage to vital macromolecules including critical proteins and DNA, causing toxicity. This toxicity has been implicated in the etiology of cardiovascular, respiratory and neurodegenerative diseases (2). In the attempt to prevent disease formation, governmental agencies regulate upper limits at which humans can safely be exposed.

The continuous exposure to toxicants seems to render us as relatively resistant to these substances, which evidences our ability to adapt. The extent of the ability to adapt defines the status of human health (3). The phenomenon of inducing an adaptive or compensatory response by a relatively mild challenge on homeostasis is called hormesis (4). To actually examine the concept of hormesis, we have selected acrolein as an exogenous compound. Acrolein, an $\alpha, \beta$-unsaturated aldehyde, is implicated in the toxicity of cigarette smoke, traffic exhaust and other air pollution (5). Acrolein can also directly induce cellular damage $(6)$ due to its intrinsic reactivity $(7,8)$. According to the Hard Soft Acid Base concept acrolein is a soft electrophile that prefers to react to soft nucleophiles such as thiols (9). The high thiol reactivity of acrolein results in Michael adducts of acrolein on cysteine residues of critical cellular proteins which can elicit cell toxicity (7). Acrolein can be considered as a toxicophore, a characteristic moiety within molecules responsible for their toxic properties, and is therefore a model for electrophilic xenobiotics that display toxicity as a direct result of their electrophilicity, e.g. NAPQI (10). In this study the concept of hormesis is investigated on a cellular level by assessing the adaptive response to acrolein in bronchial epithelial cells.

\section{Materials and Methods}

\subsection{Chemical reaction of acrolein and GSH}

The amount of acrolein (Sigma-Aldrich, St. Louis, MO, USA) reacting with reduced glutathione (GSH) (Sigma-Aldrich, St. Louis, MO, USA) was determined by adding 0, 1, 3, 6, 9, 10 and $12 \mu \mathrm{M}$ acrolein to $10 \mu \mathrm{M} \mathrm{GSH}$ at $37^{\circ} \mathrm{C}$. The amount of acrolein that has reacted with GSH is indirectly measured by quantifying the amount of GSH that has not reacted 
with acrolein. A concentration of $120 \mu \mathrm{M}$ of DTNB (Sigma-Aldrich, St. Louis, MO, USA) is added and the absorbance is spectrophotometrically determined at a wavelength of 412 $\mathrm{nm}$.

\subsection{Cell culture}

Adenovirus-12 SV40 hybrid virus transformed, non-tumorigenic human bronchial epithelial cells (BEAS-2B; ATCC, Manassas, USA) were cultured in Dulbecco's Modified Eagle's Medium (DMEM/F-12; Gibco, Bleiswijk, The Netherlands) supplemented with 50 $\mathrm{U} / \mathrm{ml}$ penicillin (Gibco, Bleiswijk, The Netherlands), $50 \mu \mathrm{g} / \mathrm{ml}$ streptomycin (Gibco, Bleiswijk, The Netherlands), $15 \mu \mathrm{g} / \mathrm{ml}$ bovine pituitary extract, $0.5 \mathrm{mg} / \mathrm{ml}$ bovine serum albumin (Invitrogen, Breda, The Netherlands), $10 \mathrm{ng} / \mathrm{ml}$ cholera-toxin (List Biological Laboratories, Inc., Campbell, California), $10 \mathrm{ng} / \mathrm{ml}$ epidermal growth factor (Merck Millipore, Darmstadt, Germany), $5 \mu \mathrm{g} / \mathrm{ml}$ insulin, $5 \mu \mathrm{g} / \mathrm{ml}$ transferrin and $0.1 \mu \mathrm{M}$ dexamethasone (Sigma-Aldrich, St. Louis, MO, USA) in an environment containing $21 \% \mathrm{O}_{2}$ and $5 \% \mathrm{CO}_{2}$ at $37^{\circ} \mathrm{C}$. The maximal passage used in this experiment was 10 .

\subsection{Treatments}

Cells were seeded in 6-well plates at a density of 400,000 cells per well and cultured overnight in $95 \% \mathrm{O}_{2}$ and $5 \% \mathrm{CO}_{2}$ at $37^{\circ} \mathrm{C}$. Treatments with acrolein were made in Hank's Balanced Salt Solution (HBSS; Gibco, Bleiswijk, The Netherlands). Cells were exposed to $0,1,3,10,30,100$ and $200 \mu \mathrm{M}$ acrolein for 30 minutes and lysed immediately to determine GSH levels. To determine cellular toxicity HBSS was replaced with medium and $24 \mathrm{~h}$ post acrolein exposure medium was collected to measure lactate dehydrogenase ( $\mathrm{LDH}$ ) activity of the released $\mathrm{LDH}$. In addition, cells were treated with $3 \mu \mathrm{M}$ and $10 \mu \mathrm{M}$ of acrolein and GSH levels were determined 4 and $6 \mathrm{~h}$ after exposure (Supplementary Figure 1). Furthermore, 4 and $8 \mathrm{~h}$ after exposure RNA was isolated to determine gammaglutamylcysteine synthetase ( $\gamma \mathrm{GCS}$ ) expression (Supplementary Figure 1). Finally, $3 \mu \mathrm{M}$ of acrolein was added to the cells $4 \mathrm{~h}$ prior to $10 \mu \mathrm{M}$ of acrolein treatment and GSH levels and cellular toxicity were measured (Supplementary Figure 1).

\subsection{Detection of cytotoxicity}

LDH activity was measured by transferring $50 \mu \mathrm{l}$ of the collected medium to a 96well plate. A solution of $100 \mathrm{mM}$ sodium pyruvate in $500 \mathrm{mM}$ potassium phosphate buffer, $\mathrm{pH} 7.5$ was prepared. After addition of $50 \mu \mathrm{l}$ of $0.25 \mathrm{mg} / \mathrm{ml} \mathrm{NADH}$ in sodium pyruvate solution, the change in absorbance at a wavelength of $340 \mathrm{~nm}$ was determined over 4 minutes using a Spectramax plate reader (SpectraMax M2 \& M2e Multi-Mode Microplate Reader, Sunnyvale, United States). LDH activity of the samples was compared to the LDH 
activity measured after LDH leakage due to cell lysis achieved by addition of $3 \%$ Triton-X100 (Sigma-Aldrich, St. Louis, MO, USA) for 15 minutes.

\subsection{Analysis of cellular GSH levels}

Cells were washed with $2 \mathrm{ml}$ 1x Dulbecco's Phosphate Buffered Saline (DPBS; Gibco, Bleiswijk, The Netherlands). The cells were lysed with $500 \mu 100 \mathrm{mM}$ potassium phosphate buffer containing 10 mM EDTA disodium salt, pH 7.5 and $1 \%$ Triton-X-100 (Sigma-Aldrich, St. Louis, MO, USA). After 30 minutes of incubation on ice, cells were scraped and then centrifuged for 10 minutes at $14,000 \mathrm{rpm} 4^{\circ} \mathrm{C}$ to remove cellular debris. The protein content was measured using the bicinchoninic acid assay (BCA; Pierce, Thermo Fisher Scientific, Etten-Leur, The Netherlands). In addition, $300 \mu$ of remaining supernatant was mixed 1:1 with $6 \%$ sulfosalicylic acid (Sigma-Aldrich, St. Louis, MO, USA) and samples were diluted 1:5 in $100 \mathrm{mM}$ potassium phosphate buffer with $10 \mathrm{mM}$ EDTA disodium salt, $\mathrm{pH}$ 7.5. Finally, GSH was determined using an enzymatic recycling method as previously described (11).

\subsection{Measurement of gamma glutamylcysteine synthetase expression}

First cells were lysed using qiazol (Qiagen, Venlo, The Netherlands). Second, 200 $\mu \mathrm{l}$ chloroform (Sigma-Aldrich, St. Louis, MO, USA) per $\mathrm{ml}$ of Qiazol was added to perform a phase separation. After incubation for $2-3$ minutes at $20^{\circ} \mathrm{C}$ and centrifugation for 15 minutes at $12,000 \mathrm{~g} 4{ }^{\circ} \mathrm{C}$, the colorless aqueous upper phase containing RNA was removed and added to $500 \mu \mathrm{l}$ isopropanol per $\mathrm{ml}$ of Qiazol to allow the RNA to precipitate. The cells incubated at $4^{\circ} \mathrm{C}$ overnight and after centrifugation the supernatant was removed. The pellet was washed with $1 \mathrm{ml} 75 \%$ ethanol per $\mathrm{ml}$ of Qiazol. After centrifugation at 7,500 $\mathrm{g}$ for 5 minutes at $4{ }^{\circ} \mathrm{C}$, the supernatant was removed and the pellet was allowed to dry for an hour. Hereafter, the pellet was resuspended in $50 \mu \mathrm{l}$ of RNAse/DNAse free water and incubated for 10 minutes at $60{ }^{\circ} \mathrm{C}$. The quantity of RNA was determined using the nanodrop (thermo scientific nanodrop 1000 spectrophotometer, isogen life science, De Meern, The Netherlands). Five hundred nanograms of RNA was converted into complementary DNA (cDNA) by using iScript cDNA synthesis kit (Biorad, Veenendaal, The Netherlands). Sensimix SYBR \& Fluorescein kit (Bioline, Alphen aan de Rijn, The Netherlands) was used to perform quantitative RT-PCR measuring gammaglutamylcysteine synthetase ( $\gamma$ GCS; sense: 5'- GCACATCTACCACGCCGTC-3' and antisense: $5^{\prime}$-CCACCTCATCGCCCCAC-3'). B-actin ( $\beta$-actin; sense: 5'- CCTGGCACCCAGCACAAT- $3^{\prime}$ and antisense: 5'-GCCGATCCACACGGAGTACT-3') was applied as housekeeping gene. Finally, the $2^{-\triangle \Delta C T}$ method was used to determine relative $\gamma$ GCS gene expression (12). 


\subsection{Statistics}

All data are shown as mean \pm SEM. A $t$ test was performed for independent samples with equal variances to assess statistical significance between two individual groups. A P $<0.05$ was considered to be statistically significant.

\section{Results}

\subsection{The reactivity of acrolein towards GSH}

To investigate the reaction of acrolein towards GSH, various amounts of acrolein were added to a fixed amount of GSH (initial concentration of $10 \mu \mathrm{M}$ in a volume of $1 \mathrm{ml}$ ) in a test tube. This revealed that acrolein reacts within seconds with GSH in stoichiometry of 1:1 (Figure 1).

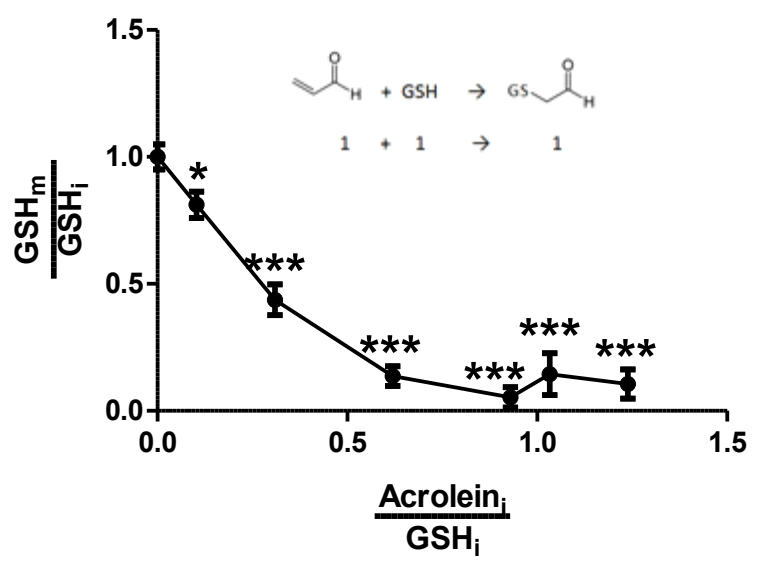

Figure 1: The chemical reaction of acrolein and GSH. Variable amounts of acrolein were added to a fixed amount of GSH (GSH ; the initial amount of GSH is $10 \mathrm{nmol}$ GSH that corresponds to $10 \mu \mathrm{M}$ GSH in $1 \mathrm{ml}$ ). Both the initial amount of acrolein $\left(\right.$ Acrolein $_{\mathrm{i}}$ ) and the amount of GSH measured $\left(\mathrm{GSH}_{m}\right)$ are shown relative to the initial added fixed amount of $\mathrm{GSH}\left(\mathrm{GSH}_{\mathrm{i}}\right)$. Experiments are at least performed in triplicate and data are shown as mean $\pm \mathrm{SEM}$. ${ }^{*} \mathrm{P}<0.05 ; * * \mathrm{P}<0.01$ and $* * * \mathrm{P}<0.001$ compared to control $(=0$ $\mu \mathrm{M}$ of acrolein). 


\subsection{Acrolein depletes GSH in the cell}

To investigate whether acrolein is quenched by intracellular GSH similarly as in the test tube, BEAS-2B cells were treated with acrolein $(1,3,10,30 \mu \mathrm{M})$, and GSH was measured. Acrolein dose-dependently reduced intracellular GSH in BEAS-2B cells (Figure 2A). The stoichiometry, i.e. reduction in the amount of GSH in the cell ( $\mathrm{nmol}$ ) relative to the amount of acrolein ( $\mathrm{nmol}$ ) added was lower compared to that in the test tube. With addition of $1 \mu \mathrm{M}$ acrolein the ratio between the amount of GSH consumed and the amount of acrolein added appeared to be $0.74: 1$ (Figure 2A). At higher concentrations of acrolein, this ratio further dropped (Figure 2A). For example, with $10 \mu \mathrm{M}$ of acrolein the ratio of GSH consumption versus acrolein added was $0.18: 1$. When compared to a $1: 1$ stoichiometry (acrolein:GSH), the relative amount of acrolein that was not captured by intracellular GSH was calculated. This calculation illustrated that as acrolein concentrations increased, the relative amount of acrolein that was not quenched by intracellular GSH also increased. When $10 \mu \mathrm{M}$ of acrolein was added to BEAS-2B cells approximately $24 \mathrm{nmol}$ of the originally added $30 \mathrm{nmol}$ of acrolein did not react with GSH (Figure $2 \mathrm{~B}$ ), which corresponds to $80 \%$ of the acrolein added.

\subsection{Acrolein induces cytotoxicity}

To identify at what threshold acrolein becomes cytotoxic, BEAS-2B cells were exposed to a range of acrolein concentrations and LDH was measured. Addition of $\leq 3 \mu \mathrm{M}$ of acrolein did not significantly induce cytotoxicity. Upon raising the concentration of acrolein above $3 \mu \mathrm{M}$, acrolein dose-dependently induced cytotoxicity, evidenced by LDH leakage (Figure $2 \mathrm{C}$ ). Relating cytotoxicity to the amount of acrolein showed that acrolein had a threshold between 22-75 fmol/cell (3-10 $\mu \mathrm{M}$ ) for inducing cytotoxicity (Figure 2D).

\subsection{GSH forms the first line of defense against acrolein induced cytotoxicity}

To determine whether GSH depletion corresponds with acrolein-induced cytotoxicity, GSH consumed was compared to the amount of LDH leakage. An association between cytotoxicity and intracellular GSH was found. It was observed that when GSH depletion exceeded $18-28 \mathrm{nmol} / \mathrm{mg}$ protein, cytotoxicity increased (Figure 2E). A decrease in the amount of GSH of $18 \mathrm{nmol} / \mathrm{mg}$ protein is seen after exposure to $3 \mu \mathrm{M}$ acrolein. No toxicity was observed after addition of $\leq 3 \mu \mathrm{M}$ of acrolein and the results indicate that all the acrolein was captured by GSH (Figure 2). At higher concentrations of acrolein only part of the acrolein was captured by GSH and toxicity was observed (Figure 2). Together these results indicate that GSH forms the first line of defense against acrolein toxicity. 

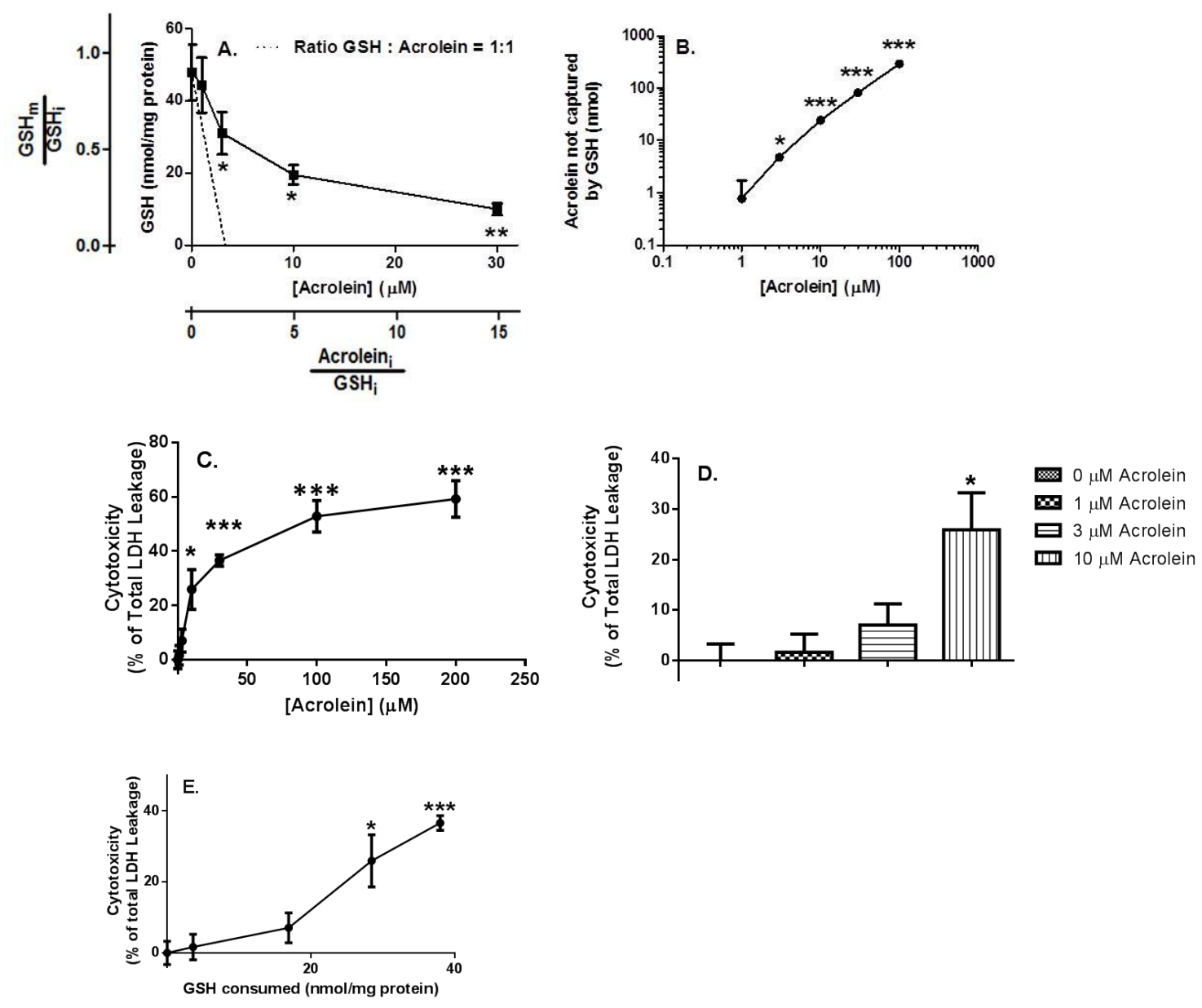

Figure 2: The effect of acrolein on GSH levels and cell toxicity of BEAS-2B cells. A) The intracellular GSH ( $\mathrm{nmol} / \mathrm{mg}$ protein) in BEAS-2B cells exposed to $0,1,3,10$ and $30 \mu \mathrm{M}$ of acrolein. The dotted line represents the theoretical reduction in intracellular GSH due to acrolein based on the chemical reaction with a stoichiometry of 1:1. Both the initial amount of acrolein $\left(\right.$ Acrolein $\left._{\mathrm{i}}\right)$ relative to the initial intracellular amount of $\mathrm{GSH}\left(\mathrm{GSH}_{\mathrm{i}}\right)$ as well as the added concentration of acrolein are shown. Both the amount of GSH measured after the exposure $\left(\mathrm{GSH}_{\mathrm{m}}\right)$ relative to the initial intracellular amount of $\mathrm{GSH}$ $\left(\mathrm{GSH}_{\mathrm{i}}\right)$ as well as the amount of $\mathrm{GSH}$ (nmol/mg protein) are depicted. B) The absolute amount of acrolein not reacted with intracellular GSH relative to the concentration of acrolein added. C) Cytotoxicity in BEAS-2B cells exposed to 0, 1, 3, 10, 30, 100 and 200 $\mu \mathrm{M}$ of acrolein. Cytotoxicity is expressed as a percentage of LDH activity observed after 
cell lysis (addition of $3 \%$ Triton-X-100) and shown relative to cytotoxicity observed at exposure to $0 \mu \mathrm{M}$ of acrolein. D) Cytotoxicity in BEAS-2B cells exposed to $0,1,3,10 \mu \mathrm{M}$ of acrolein. E) Cytotoxicity plotted against the reduction in intracellular amount of GSH. $\mathrm{N}=4$ and data are shown as mean $\pm \mathrm{SEM}$. ${ }^{*} \mathrm{P}<0.05 ; * * \mathrm{P}<0.01$ and $* * * \mathrm{P}<0.001$ compared to control ( $=0 \mu \mathrm{M}$ of acrolein).

\subsection{Adaptation to acrolein}

To assess whether cells can adapt to acrolein, cells were pretreated with $3 \mu \mathrm{M}$ acrolein $4 \mathrm{~h}$ prior to a second exposure of $10 \mu \mathrm{M}$ acrolein. As also shown above, treatment of control cells to $3 \mu \mathrm{M}$ of acrolein alone did not significantly increase cytotoxicity, whereas $10 \mu \mathrm{M}$ of acrolein did significantly increased the observed cytotoxicity compared to cytotoxicity in control cells (Figure $3 \mathrm{~A}$ ). In cells pretreated with $3 \mu \mathrm{M}$ of acrolein, the cytotoxicity of $10 \mu \mathrm{M}$ of acrolein was significantly diminished (Figure $3 \mathrm{~A}$ ).

Exposure of BEAS-2B cells to $3 \mu \mathrm{M}$ acrolein immediately decreased the amount of GSH (Figure 3B). Furthermore, exposure to $10 \mu \mathrm{M}$ of acrolein directly reduced intracellular GSH levels significantly more (Figure $3 \mathrm{~B}$ ). Moreover, pretreatment with the non-toxic concentration of acrolein $(3 \mu \mathrm{M})$ prevented a reduction of intracellular GSH by $10 \mu \mathrm{M}$ of acrolein (Figure 3B).

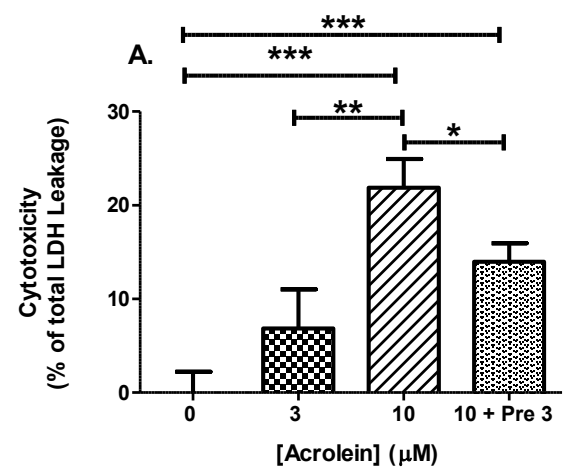

B.

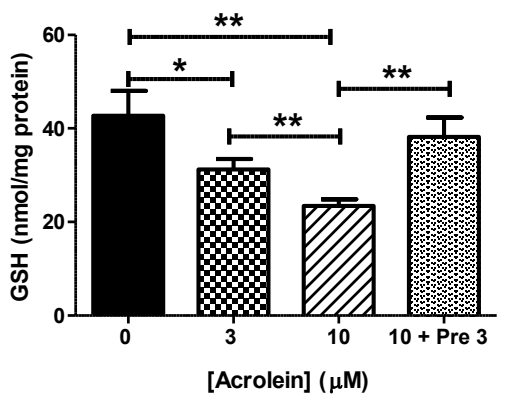

Figure 3: Effect of pretreatment of BEAS-2B cells with $3 \mu \mathrm{M}$ of acrolein on cytotoxicity (A) and GSH levels (B) induced by $10 \mu \mathrm{M}$ of acrolein. $\mathrm{N} \geq 6$ and data are shown as mean \pm SEM. $* \mathrm{P}<0.05 ; * * \mathrm{P}<0.01$ and $* * * \mathrm{P}<0.001$ compared to control (= $0 \mu \mathrm{M}$ of acrolein). 


\subsection{Acrolein-induced adaptation involves GSH induction}

To assess whether cellular adaptation to acrolein involved GSH induction, cellular GSH levels and $\gamma$ GCS gene expression were analyzed at different times. Exposure of BEAS$2 \mathrm{~B}$ cells to either 3 or $10 \mu \mathrm{M}$ acrolein resulted in a rapid but transient reduction of intracellular GSH levels (Figure 4A and B). Within approximately $3 \mathrm{~h}$ the amount of GSH returned to and increased significantly above baseline (Figure $4 \mathrm{~A}$ and $\mathrm{B}$ ). Exposure to acrolein also resulted in an increase in $\gamma \mathrm{GCS}$ mRNA expression (Figure $4 C$ and $D$ ).

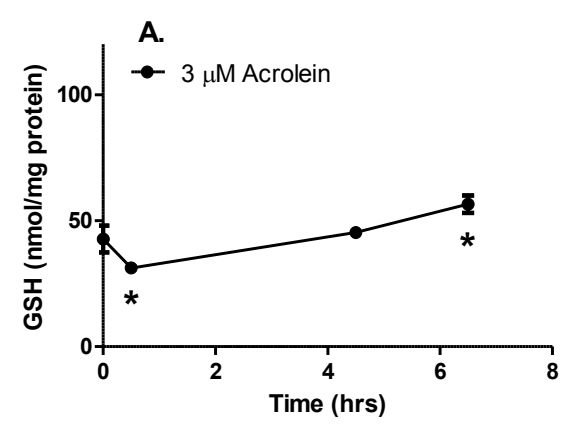

C.

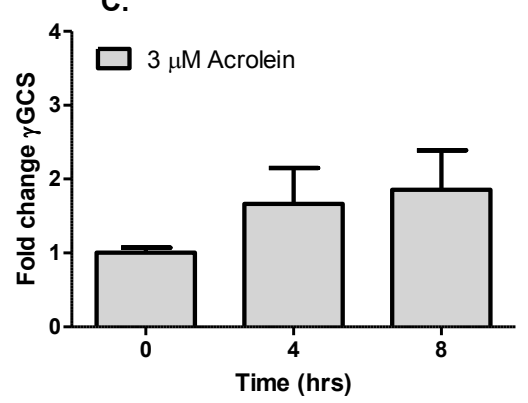

B.

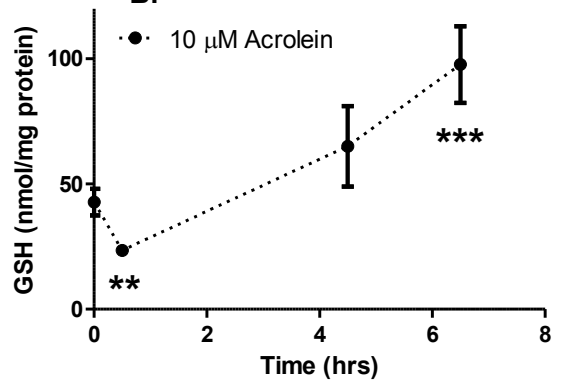

D.

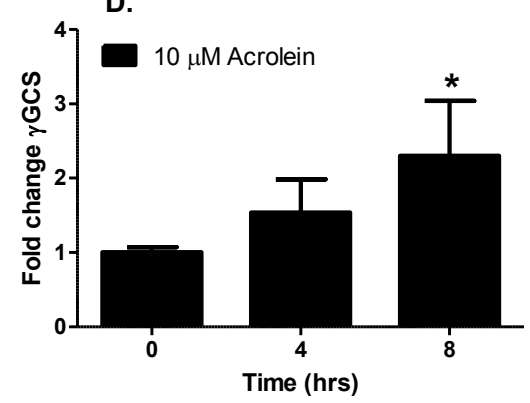

Figure 4: The time course of GSH content in BEAS-2B cells after addition of $3 \mu \mathrm{M}(\mathrm{A})$ or $10 \mu \mathrm{M}(\mathrm{B})$ of acrolein. The expression yGCS in BEAS-2B cells exposed to $3 \mu \mathrm{M}(\mathrm{C})$ and 10 $\mu \mathrm{M}(\mathrm{D})$ of acrolein. $\gamma \mathrm{GCS}$ mRNA expression is shown relative to $\beta$-actin. $N \geq 3$ and data are shown as mean \pm SEM. $* \mathrm{P}<0.05 ; * * \mathrm{P}<0.01$ and $* * * \mathrm{P}<0.001$ compared to control $(\mathrm{T}=0)$. 


\section{Discussion}

Hormesis is a theoretical concept in which it is suggested that exposure to a low dose of a chemical that is damaging at higher doses, induces an adaptive upgrade of the cellular protection $(4,13)$. If this theory is correct it has important ramifications for risk assessment and our perception of toxicity. This prompted us to investigate the hormesis concept and we used the $\alpha, \beta$-unsaturated aldehyde acrolein.

Acrolein is a soft electrophile that prefers to react with soft nucleophiles, which in biological systems are predominantly thiols (8). Acrolein directly induces cellular toxicity and is a model for electrophilic xenobiotics.

A cell is protected against electrophile-induced damage (14). In BEAS-2B cells exposed to $\leq 3 \mu \mathrm{M}$ of acrolein, the acrolein is efficiently captured by GSH thus preventing cytotoxicity. We confirmed in the test tube that acrolein reacts with GSH within seconds having a stoichiometry of $1: 1$. Together this indicates that the nucleophilic tripeptide GSH is intracellularly the first line of defense against acrolein.

Within the cell, the capacity of GSH to quench acrolein appears to be limited. Upon raising the concentration of acrolein above $3 \mu \mathrm{M}$, GSH only captures part of the acrolein, which coincides with cytotoxicity apparent by LDH leakage. This observed acrolein-induced toxicity can be explained by adduction of critical thiol groups in proteins by the acrolein not captured by GSH (15). This highlights the crucial role of GSH in the prevention of acrolein toxicity.

To study hormesis, the cells were first treated with a non-toxic concentration of 3 $\mu \mathrm{M}$ acrolein. This pretreatment appeared to reduce the cytotoxicity induced by a second exposure to a higher normally toxic concentration of acrolein $(10 \mu \mathrm{M})$. This demonstrates that the pretreatment with the low dose induces an adaptation. To further study this adaptive response of the cell to acrolein, we examined the molecular mechanism.

The crucial role of GSH in the prevention of acrolein toxicity prompted us to quantify GSH levels in the cell. After the expected initial decrease, an increase in the intracellular GSH concentration was found by the hormetic treatment with $3 \mu \mathrm{M}$ acrolein. Upon a second exposure of $10 \mu \mathrm{M}$ acrolein, the GSH concentration did not drop below control level. Apparently, acrolein pretreatment protects against the further depletion of GSH.

The hormetic exposure enforces the GSH defense. The rate limiting step in GSH synthesis is catalyzed by the enzyme GCS (16). Kelch-like ECH-associated protein 1 (Keap1) is known to be adducted by acrolein (17), which results in translocation of nuclear factor (erythroid-derived 2)-like 2 (Nrf2) to the nucleus (18), where it promotes the gene expression of antioxidant defense enzymes including yGCS (19). Our results indeed show that $\gamma$ GCS expression increases. 
Toxicity of acrolein appears to be alleviated by small hormetic dosages. This exemplifies the dynamics of cellular protection towards acrolein. This cellular flexibility is not acknowledged in risk assessment procedures. Nevertheless, hormesis does affect xenobiotic toxicity. A cell is triggered to enforce its protection before a specific hazard might become an imminent danger. The cell appears to be flexible in its defense and raises its specific shields when provoked. An adaptive strategy is far more economical and efficient than continuously wearing a "suit of armour" against all possible threats. Thus the challenge is to correctly incorporate hormesis in risk assessment to accurately assess toxicity. The paradigm of risk assessment is rigid, while in reality cells are flexible.

\section{Supplement}

\subsection{Supplementary Material and Methods}

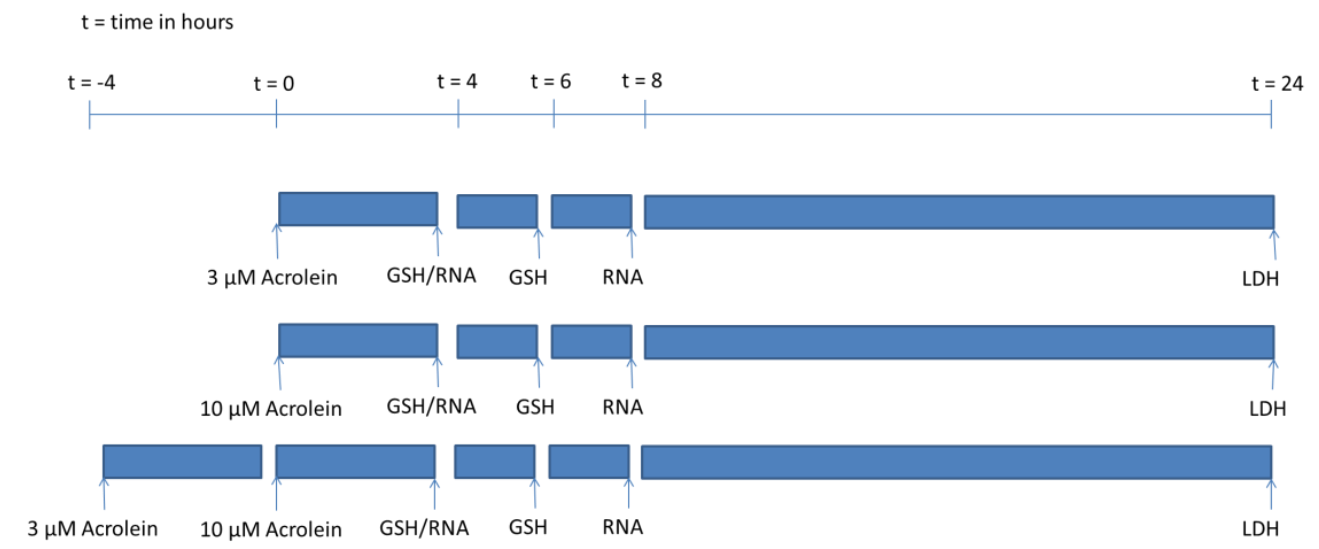

Supplementary Figure 1: Timeline including acrolein exposure and GSH, RNA and LDH measurements. 


\section{References}

1. : Agency for Toxic Substances and Disease Registry; 2011; Agency for Toxic Substances and Disease Registry]. Available from: http://www.atsdr.cdc.gov/substances/indexAZ.asp.

2. Bandyopadhyay UD, D; Banerjee, RK. Reactive oxygen species: Oxidative damage and pathogenesis. Current Science. 1999;77(5).

3. What is health? The ability to adapt. Lancet. [Editorial]. 2009 Mar 7;373(9666):781.

4. Calabrese EJ, Baldwin LA. Defining hormesis. Human \& experimental toxicology. $2002 \mathrm{Feb} ; 21(2): 91-7$.

5. Stevens JF, Maier CS. Acrolein: sources, metabolism, and biomolecular interactions relevant to human health and disease. Molecular nutrition \& food research. [Research Support, N.I.H., Extramural Review]. 2008 Jan;52(1):7-25.

6. Haenen GR, Vermeulen NP, Tai Tin Tsoi JN, Ragetli HM, Timmerman H, Bast A. Activation of the microsomal glutathione-S-transferase and reduction of the glutathione dependent protection against lipid peroxidation by acrolein. Biochemical pharmacology. 1988 May 15;37(10):1933-8.

7. Randall MJ, Hristova $M$, van der Vliet $A$. Protein alkylation by the alpha,beta-unsaturated aldehyde acrolein. A reversible mechanism of electrophile signaling? FEBS letters. 2013 Nov 29;587(23):3808-14.

8. Esterbauer $\mathrm{H}$, Schaur RJ, Zollner $\mathrm{H}$. Chemistry and biochemistry of 4hydroxynonenal, malonaldehyde and related aldehydes. Free radical biology \& medicine. [Research Support, Non-U.S. Gov't Review]. 1991;11(1):81-128.

9. Ho TL, Ho HC, Hamilton LD. Biochemical significance of the hard and soft acids and bases principle. Chemico-biological interactions. [Review]. 1978 Oct;23(1):65-84.

10. Nassini R, Materazzi S, Andre E, Sartiani L, Aldini G, Trevisani M, et al. Acetaminophen, via its reactive metabolite $\mathrm{N}$-acetyl-p-benzo-quinoneimine and transient receptor potential ankyrin-1 stimulation, causes neurogenic inflammation in the airways and other tissues in rodents. FASEB journal : official publication of the Federation of American Societies for Experimental Biology. [Research Support, Non-U.S. Gov't]. 2010 Dec;24(12):4904-16. 
11. Rahman I, Kode A, Biswas SK. Assay for quantitative determination of glutathione and glutathione disulfide levels using enzymatic recycling method. Nature protocols. 2006;1(6):3159-65.

12. Livak KJ, Schmittgen TD. Analysis of relative gene expression data using real-time quantitative PCR and the 2(-Delta Delta C(T)) Method. Methods. 2001 Dec;25(4):402-8.

13. Mattson MP. Hormesis defined. Ageing Res Rev. [Research Support, N.I.H., Intramural Review]. 2008 Jan;7(1):1-7.

14. Circu ML, Aw TY. Reactive oxygen species, cellular redox systems, and apoptosis. Free radical biology \& medicine. [Research Support, N.I.H., Extramural Review]. 2010 Mar 15;48(6):749-62.

15. Cai J, Bhatnagar A, Pierce WM, Jr. Protein modification by acrolein: formation and stability of cysteine adducts. Chemical research in toxicology. [Research Support, N.I.H., Extramural Research Support, Non-U.S. Gov't]. 2009 Apr;22(4):708-16.

16. Rahman I. Regulation of glutathione in inflammation and chronic lung diseases. Mutation research. [Research Support, N.I.H., Extramural Research Support, Non-U.S. Gov't Review]. 2005 Nov 11;579(1-2):58-80.

17. McMahon M, Lamont DJ, Beattie KA, Hayes JD. Keap1 perceives stress via three sensors for the endogenous signaling molecules nitric oxide, zinc, and alkenals. Proceedings of the National Academy of Sciences of the United States of America. [Research Support, Non-U.S. Gov't]. 2010 Nov 2;107(44):18838-43.

18. Kwak MK, Kensler TW, Casero RA, Jr. Induction of phase 2 enzymes by serum oxidized polyamines through activation of Nrf2: effect of the polyamine metabolite acrolein. Biochemical and biophysical research communications. [Research Support, U.S. Gov't, P.H.S.]. 2003 Jun 6;305(3):662-70.

19. Cho HY, Reddy SP, Kleeberger SR. Nrf2 defends the lung from oxidative stress. Antioxidants \& redox signaling. [Review]. 2006 Jan-Feb;8(1-2):76-87. 


Chapter 4

Silver nanoparticles induce hormesis in

A549 human epithelial cells.

Mireille M.J.P.E. Sthijns, Waluree Thongkam, Catrin Albrecht, Bryan Hellack, Aalt Bast, Guido R.M.M. Haenen, Roel P.F. Schins.

Toxicology in vitro. 2017 Jan 18;40:223-233. 


\section{Abstract}

Despite the gaps in our knowledge on the toxicity of silver nanoparticles (AgNPs), the application of these materials is fast expanding, from medicine, to food as well as the use in consumer products. It has been reported that prolonged exposure might make cells more resistant to AgNPs. This prompted us to investigate if AgNPs may give rise to a hormetic response. Two types of AgNPs were used, i.e. colloidal AgNPs and an AgNP powder. For both types of nanosilver it was found that a low dose pretreatment of A549 human epithelial cells with AgNPs induced protection against a toxic dose of AgNPs and acrolein. This protection was more pronounced after pretreatment with the colloidal AgNPs. Interestingly, the mechanism of the hormetic response appeared to differ from that of acrolein. Adaptation to acrolein is related to Nrf2 translocation, increased mRNA expression of $\mathrm{YGCS}, \mathrm{HO}-1$ and increased GSH levels and the increased GSH levels can explain the hormetic effect. The adaptive response to AgNPs was not related to an increase in mRNA expression of $y$ GCS and GSH levels. Yet, HO-1 mRNA expression and Nrf2 immunoreactivity were enhanced, indicating that these processes might be involved. So, AgNPs induce adaptation, but in contrast to acrolein GSH plays no role. 


\section{Introduction}

Silver nanoparticles (AgNPs) have found increasing applications in industry, medicine and consumer products and their use is fast expanding $(1,2)$. The main reason why silver is used in medical settings is because of its antibiotic properties (3). Nevertheless, there are fundamental gaps in the knowledge on their toxicity and mode of action (4-11). It is long known, that the adverse health effects of toxic fine particles like crystalline silica dust and asbestos are linked to reactive oxygen species (ROS) formation and induction of oxidative stress. Subsequently, redox dependent cellular processes and effects became the focus of investigations on the toxicity of ambient and engineered nanoparticles $(12,13)$. Indeed, also studies with AgNPs relate toxicity to their ability to generate ROS and to induce oxidative stress $(1,14-17)$. Paradoxically, AgNPs also activate the transcription factor nuclear factor erythroid-derived 2 related factor 2 (Nrf2) (16, 1821). This leads to upregulation of cellular antioxidant and detoxification enzymes and upgrades the cellular protection against oxidative stress $(17,22)$.

This prompted us to examine the potential relevance of both processes induced by AgNPs i.e. increased formation of ROS versus upregulation of the antioxidant defense. In recent years, peculiar findings on the toxicity of AgNPs have been reported. AudeGarcia et al. compared the toxicity of AgNPs administered as a single high dose versus the same amount of AgNPs administered as repeated low doses on primary mouse macrophages (23). Intracellular accumulation of $\mathrm{Ag}$ was similar for both treatment regimes, while only the single high dose treatment caused a pro-inflammatory activation of these cells. Brzoska and co-workers reported a short term (2 h) increase in DNA damage returning to baseline after prolonged exposure ( $24 \mathrm{~h}$ ) to AgNPs in HepG2 liver epithelial cells (24). This may point to a hormetic response to AgNPs. Hormesis is that "exposure to a low dose of a chemical that is damaging at higher doses, induces an adaptive upgrade of cellular protection" (25). In recent years, this adaptation concept has also gained more attention for nanoparticles $(26,27)$.

The process of hormesis can be illustrated with the ubiquitous environmental pollutant acrolein. Recently, we showed that in BEAS-2B lung epithelial cells a low dose of acrolein induces protection against a high toxic dose of acrolein $(28,29)$. Acrolein can react with thiols of important cellular proteins, causing toxicity. The protagonist in the protective mechanism induced by this reactive unsaturated aldehyde is the thiol containing compound glutathione (GSH). GSH levels are upregulated by a low dose of acrolein via increasing the Nrf2-mediated gene expression of the rate limiting enzyme of GSH synthesis gamma-glutamylcysteine synthetase ( $\gamma \mathrm{GCS}$ ) (30). This protects against a second exposure to acrolein and thus gives the hormetic response. 
The aim of our study was to investigate the hormetic adaptive response to AgNPs by investigating whether pretreatment with a low dose of AgNPs can protect against exposure to a high dose. Two contrasting types of AgNPs, i.e. colloidal versus powder, were selected and characterized for primary particle size, solubility and agglomeration behavior in this study. Additionally, the mechanism of adaptation was compared to acrolein. Experiments were performed in human A549 lung epithelial cells, which have been widely used to investigate the toxicity of various types of engineered nanoparticles including AgNPs $(6,14,15,24,31-34)$. The use of this cell line also has practical relevance due to inhalation exposure to nanosilver caused by a growing number of consumer products such as desinfectants, deodorants, antimicrobial sprays and other applications where these particles also become airborne $(9,35)$. Despite its potential limitations (i.e. transformation status), A549 cells were used in several recent and ongoing large-scale nanosafety projects to allow for the necessary bridging of data from different research labs. Their robustness for toxicity evaluation of nanomaterials was evaluated in a round robin approach within the EU-7 $7^{\text {th }}$ framework projects ENPRA (36).

\section{Materials and Methods}

\subsection{Nanomaterials}

Two types of silver nanoparticles were used, referred to in this study as AgNP1 and AgNP2. The first material, AgNP1, was purchased from Skyspring Nanomaterials, Inc. (US). This material is available as a powder. The AgNP2 represents a sample of the NM-300 reference nanomaterial and was received from the European Commission Joint Research Centre (Ispra, Italy) in the context of the EU FP7 project ENPRA. The nanomaterial NM-300 is a dispersion of silver nanoparticles in deionised water (85\%) with $7 \%$ stabilising agent (ammonium nitrate) and $8 \%$ emulsifiers (4\% each of polyoxyethylene glycerol Trioleate and Tween 20). This colloidal nanosilver samples were purchased and studied along with its dispersant control (i.e. NM-300dis) within the framework of the ENPRA project (36). Because of absence of cytotoxicity for NM-300dis towards the A549 cells up to the highest test concentration equivalent to $80 \mu \mathrm{g} / \mathrm{cm}^{2}$ for NM-300 (data not shown), it was decided not to include this dispersant control further in the present study. Representative scanning electron microscopy (SEM) images of both AgNPs are shown in Figure 1. As can be seen in the figure, the AgNP1 consists of compact, near-spherical to elongated primary particles which tend to form compact agglomerates/aggregates. Detailed SEM analysis of the material revealed a primary particle size of $37.0 \mathrm{~nm} \pm 13.0 \mathrm{~nm}$. In contrast, the AgNP2 consists of homogenously distributed, merely spherical particles. For this sample a primary particle size of $16.6 \mathrm{~nm} \pm 4.4 \mathrm{~nm}$ was determined. Both types of nanoparticles also revealed contrasting dissolution properties. We determined the solubility of the samples 
in deionised water (at room temperature) after continuous shaking, followed by syringe filtration and analysis by ICP-OES. Upon $72 \mathrm{~h}$ incubation, for the AgNP1 about $0.2 \%$ was found to be dissolved, whereas for the AgNP2 sample this was much larger, i.e. up to $5 \%$.

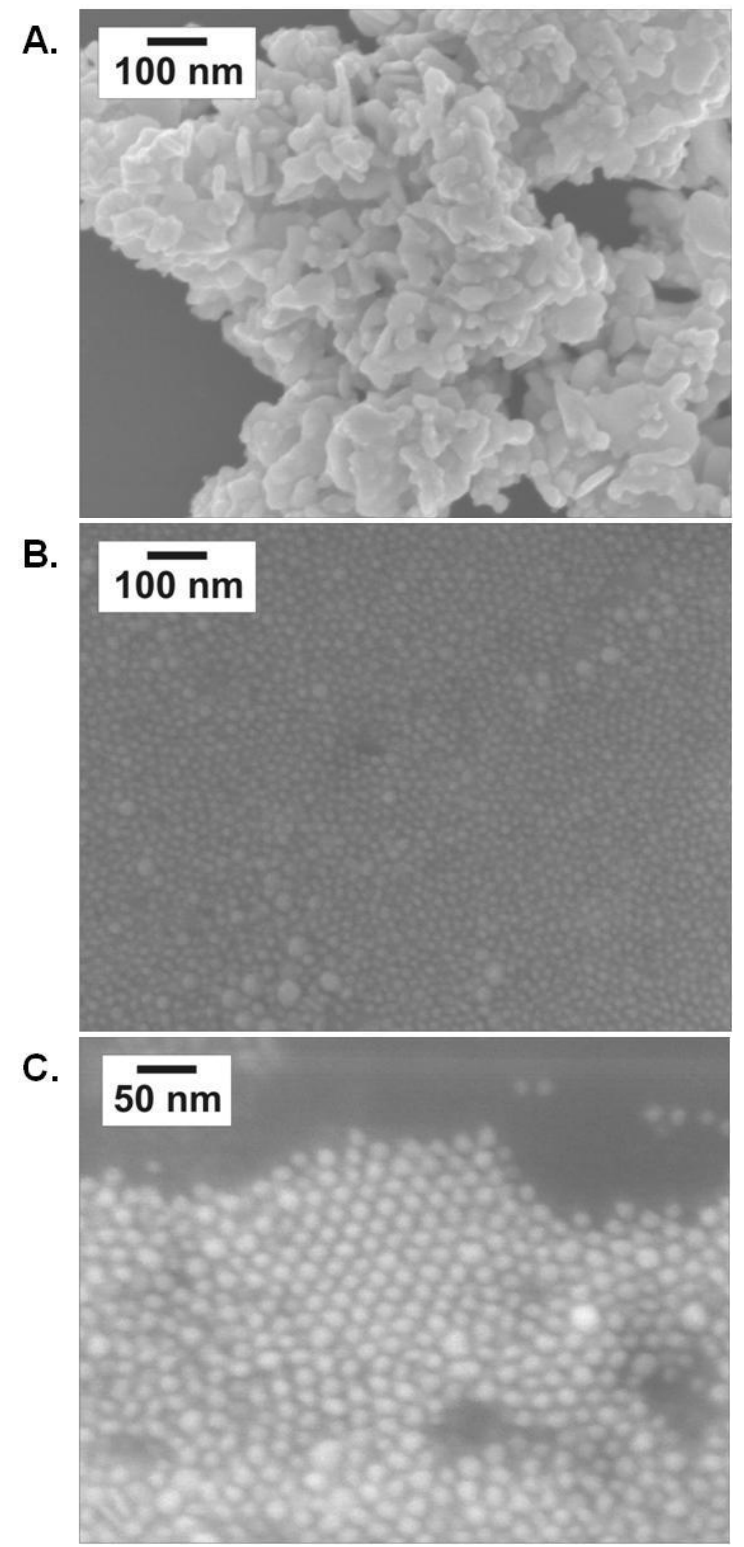

Figure 1: Representative scanning electron micrographs of AgNP1 (A) and AgNP2 (B and C). 


\subsection{Culture and treatment of cells}

Human lung adenocarcinoma cells (A549) were cultured in Dulbecco's Modified Eagle Medium (DMEM)/F12 (Invitrogen, Bleiswijk, The Netherlands) supplemented with $10 \%$ fetal calf serum (FCS, Sigma-Aldrich, St. Louis, MO, USA) and $1 \%$ penicillin/streptomycin (Life Technologies, Bleiswijk, The Netherlands) in an environment containing $5 \% \mathrm{CO}_{2}$ and $95 \%$ air at $37{ }^{\circ} \mathrm{C}$. Experiments were performed with cells from passage $15-20$.

All particle suspensions for the in vitro experiments were prepared on the basis of the nanoparticle dispersion protocols as developed within the EU- $7^{\text {th }}$ framework project ENPRA (http://www.enpra.eu) $(36,37)$ and the SIINN-ERANET project NanOxiMet (http://www.nanoximet.eu) with following specific modifications: Stock solutions of 1 $\mathrm{mg} / \mathrm{ml}$ were prepared in sterile RNase free water with $2 \%$ adult bovine serum (ABS, Sigma-Aldrich, Darmstadt, Germany). The suspensions were then sonicated for $10 \mathrm{~min}$ with a Branson 450 Sonifier with Cuphorn at a power of 5.71 (200 W) and $20 \%$ duty cycle. The stock suspensions were quickly further diluted in exposure medium to a concentration of $256 \mu \mathrm{g} / \mathrm{ml}$, equivalent to the treatment concentration of $80 \mu \mathrm{g}$ nanoparticles per $\mathrm{cm}^{2}$ of cell culture monolayer. The exposure medium for the AgNP samples consisted of phenol red free DMEM/F12 with 1 \% 4-(2-Hydroxyethyl)piperazine-1-ethanesulfonic acid, N-(2Hydroxyethyl)piperazine- $\mathrm{N}^{\prime}$-(2-ethanesulfonic acid) (HEPES, Sigma-Aldrich, Darmstadt, Germany) and $1 \%$ penicillin/streptomycin. The acrolein (Sigma-Aldrich, St. Louis, MO, USA) exposures were performed in Hank's Balanced Salt Solution (HBSS, Gibco, Bleiswijk, The Netherlands). For dose response relationship evaluations, the $\mathrm{A} 549$ cells were exposed to $0,1,3,10,30,100$ and $200 \mu \mathrm{M}$ acrolein for $30 \mathrm{~min}$ and to $0,1.25,2.5,5,10,20,40,80$ $\mu \mathrm{g} / \mathrm{cm}^{2}$ AgNPs for $24 \mathrm{~h}$, respectively. Toxicity and intracellular GSH levels were then determined as described in the subsequent sections.

To investigate adaptation processes, repeated exposures were performed at concentrations and treatment times based on the outcome of initially performed dose response analysis, and, for acrolein, in part also on the basis of earlier observations in BEAS-2B cells (28). Details of these treatment protocols are shown in Supplementary Figure 1. Briefly, the A549 cells were pretreated with $10 \mu \mathrm{M}$ acrolein for 30 min after which the HBSS-containing acrolein was replaced with exposure medium. After $3.5 \mathrm{~h}$ incubation, a second dose of $100 \mu \mathrm{M}$ acrolein was then added and cytoxicity and intracellular GSH levels were determined. For AgNP2, a pretreatment of $5 \mu \mathrm{g} / \mathrm{cm}^{2}$ was used and after $24 \mathrm{~h}$ of exposure, either a second AgNP2 dose of $80 \mu \mathrm{g} / \mathrm{cm}^{2}$ or $100 \mu \mathrm{M}$ acrolein was added. Acrolein was incubated for 30 minutes, while particles were incubated for $24 \mathrm{~h}$ after which again cytotoxicity and GSH levels were measured 
(Supplementary Figure 1). For AgNP1, the same experimental set-up as for AgNP2 was performed, however in this case the pretreatment concentration was $2.5 \mu \mathrm{g} / \mathrm{cm}^{2}$ and the second treatment was $60 \mu \mathrm{g} / \mathrm{cm}^{2}$. For the mRNA expression analyses, A549 cells were exposed for $8 \mathrm{~h}$ to suspensions of AgNP1 and AgNP2 at the respective concentrations of $2.5 \mu \mathrm{g} / \mathrm{cm}^{2}$ and $5 \mu \mathrm{g} / \mathrm{cm}^{2}$. For acrolein, cells were treated at $10 \mu \mathrm{M}$ in HBSS for $30 \mathrm{~min}$ and subsequent further incubation for 7.5 in fresh exposure medium. For the immunohistochemistry evaluation, cells were exposed for $4 \mathrm{~h}$ to $2.5 \mu \mathrm{g} / \mathrm{cm}^{2}$ (AgNP1) or 5 $\mu \mathrm{g} / \mathrm{cm}^{2}$ (AgNP2), or for $30 \mathrm{~min}$ to acrolein $(10 \mu \mathrm{M})$ in HBSS.

\subsection{Characterization of the AgNP dispersions by dynamic light scattering analysis}

We evaluated the dispersion states of the AgNP1 and AgNP2 by dynamic light scattering (DLS) using a Delsa-Nano C (Beckman Coulter Inc., Krefeld, Germany). The measurements were performed on three different types of suspensions. To verify the effectiveness of the applied sonication protocol, freshly prepared, sonicated stock suspensions of the nanoparticles (i.e $1 \mathrm{mg} / \mathrm{ml}$ water containing $2 \% \mathrm{ABS}$ ) were analyzed. To evaluate the dispersion states of the AgNPs under the applied cell culture testing conditions, we also measured the diluted AgNP suspensions containing the phenol red free DMEM/F12 with $10 \%$ FCS and $1 \%$ penicillin/streptomycin. Finally, to evaluate the impact of serum on the agglomeration status of the nanoparticles, we analyzed the diluted culture medium dispersions without $10 \%$ FCS. The results of these complementary analyses are shown in Table 1. The two samples differed in their aggregate/agglomerate size in the various tested fluids. Under cell treatment conditions (i.e. 10 \% FCS containing medium), the Z-averages (hydrodynamic diameters) were $642 \mathrm{~nm}$ for AgNP1 and $408 \mathrm{~nm}$ for AgNP2, respectively. The relative high polydispersity index (PDI) for both materials under these conditions, suggests that the A549 cells were exposed to agglomerates with a rather wide range of diameters.

Table 1: Characterisation of the silver nanoparticle dispersions by dynamic light scattering (DLS)

\begin{tabular}{|c|c|c|c|}
\hline & Sonicated suspension $^{\mathrm{a}}$ & ${\text { Medium }-\mathrm{FCS}^{\mathrm{b}}}$ & ${\text { Medium }+\mathrm{FCS}^{\mathrm{c}}}$ \\
\hline AgNP1 & $\begin{array}{c}690.6 \pm 74.5 \\
\text { (PDI:0.298 } \pm 0.02)\end{array}$ & $\begin{array}{c}548.1 \pm 100.3 \\
\text { (PDI: } 0.396 \pm 0.01)\end{array}$ & $\begin{array}{c}642.1 \pm 230.2 \\
\text { (PDI: } 0.317 \pm 0.04)\end{array}$ \\
\hline AgNP2 & $242.5 \pm 93.5$ & $138.6 \pm 48.3$ & $408.5 \pm 108.6$ \\
& (PDI: $0.151 \pm 0.00)$ & (PDI: $0.174 \pm 0.05)$ & (PDI: $0.243 \pm 0.01)$ \\
\hline
\end{tabular}


The diameter is expressed as the Z-average hydrodynamic diameter $(\mathrm{nm}) \pm$ standard deviation $(\mathrm{n}=3) ; \mathrm{PDI}=$ polydispersity index. ${ }^{\mathrm{a}}$ Sterile RNAse free water supplemented with $2 \%$ ABS, measured after sonication; ${ }^{b}$ Phenol red free DMEM/F12 medium supplemented with $1 \%$ penicillin/streptomycin (without FCS); ${ }^{C}$ Phenol red free DMEM/F12 medium supplemented with $10 \%$ FCS and $1 \%$ penicillin/streptomycin, as used in cell culture experiments.

\subsection{Cytotoxicity}

Cytotoxicity was evaluated using the WST-1 assay (Roche, Mannheim, Germany). The assay is based on the cleavage of the tetrazolium salt WST-1 to formazan by cellular mitochondrial dehydrogenases. The amount of formazan directly correlates to the number of living cells in the culture. A549 cells were seeded in 96 well plates at a density of at 10 000 cells/well and incubated for $24 \mathrm{~h}$. After treatment of the cells $10 \mu \mathrm{l}$ of the WST-1 reagent was added to each well (1:10 dilution), and the plates were then incubated for a further $30 \mathrm{~min}$ at $37^{\circ} \mathrm{C}$. Absorbance was measured at $450 \mathrm{~nm}$ by microplate reader (Infinite 200 pro NanoQuant, Tecan). The treatment protocol for the WST-1 assay included evaluation of assay artefacts by analysis of additional control wells as developed in the ENPRA project (36). Artefacts were not observed with the WST-1 assay, unlike the lactate dehydrogenase (LDH) cytotoxicity test, where assay interference could not be ruled out (data not shown).

\subsection{Intracellular GSH levels}

Cells were seeded at a density of 300000 cells/well in 6 -well plates. Thereafter, cells were treated as described above. Then, cells were washed using $2 \mathrm{ml}$ phosphate buffered saline (PBS, Sigma-Aldrich, Darmstadt, Germany). In addition, $500 \mu$ lysis buffer (0.1 M Potassium Phosphate buffer containing 10 mM EDTA disodium salt, $\mathrm{pH} 7.5$ and $1 \%$ Triton-X-100 (Sigma-Aldrich, St. Louis, MO, US)) was added to the cells and they were incubated for $30 \mathrm{~min}$ on ice. After scraping the cells, lysates were transferred to eppendorf tubes that were centrifuged for $10 \mathrm{~min}$ at $14000 \mathrm{rpm}$ and $4{ }^{\circ} \mathrm{C}$ to get rid of cellular debris. Part of the supernatant was used to determine protein content using the bicinchoninic acid assay (BCA; Pierce, Thermo Fisher Scientific, Etten-Leur, The Netherlands). Finally, $6 \%$ sulfosalicylic acid (Sigma-Aldrich, St. Louis, MO, USA) was added in a ratio of 1:1 to $300 \mu \mathrm{l}$ of the remaining supernatant and the mixture was diluted 1:5 in $0.1 \mathrm{M}$ lysis buffer without Triton-X-100. The previously described enzymatic recycling method was used to determine GSH levels (38). 


\subsection{Expression of Heme Oxygenase-1 and gamma-glutamylcysteine synthetase}

Cells were plated at a density of 300000 cells/well $24 \mathrm{~h}$ before the start of the exposure. After treatment, cells were lysed using $500 \mu \mathrm{l}$ qiazol (Qiagen, Venlo, The Netherlands). Phase separation was induced by addition of $100 \mu \mathrm{l}$ chloroform (SigmaAldrich, St. Louis, MO, US) for $2-3 \mathrm{~min}$ at $20^{\circ} \mathrm{C}$ and samples were then centrifuged (15 $\mathrm{min}$, $12000 \mathrm{~g}$ ) at $4{ }^{\circ} \mathrm{C}$. The upper phase was then collected and supplemented with $250 \mu \mathrm{l}$ of isopropanol (Sigma-Aldrich, St. Louis, MO, US). After overnight incubation at $4{ }^{\circ} \mathrm{C}$ followed by centrifugation, supernatants were discarded and pellets were washed in $0.5 \mathrm{ml} 75 \%$ ethanol (Sigma-Aldrich, St. Louis, MO, US). Samples were then centrifuged for $5 \mathrm{~min}$ at 7 $500 \mathrm{~g}$ and $4{ }^{\circ} \mathrm{C}$ upon which supernatants were removed. Pellets were allowed to dry at room temperature for $1 \mathrm{~h}$ and then resuspended in $50 \mu \mathrm{l}$ RNase/DNase free water. Resuspended samples were incubated for $10 \mathrm{~min}$ at $60{ }^{\circ} \mathrm{C}$ and the amount of RNA was measured using the nanoDrop (Thermo scientific nanodrop 1000 spectrophotometer, isogen life science, De Meern, The Netherlands). Hereafter, complementary DNA (cDNA) was made of 500 ng of RNA using the iScript cDNA synthesis kit (Biorad, Veenendaal, The Netherlands). Quantitative RT-PCR was performed in Bio Rad MyiQ Real-Time PCR Detection System (American Laboratory Trading, Boston/Cambridge) using the $\mathrm{iQ}^{\mathrm{TM}} \mathrm{SYBR}^{\circledR}$ Green Supermix (Biorad) with the following primers: Heme oxygenase-1 (HO-1; sense: 5'CTTCTTCACCTTCCCCAACA-3' and antisense: 5'-GCTCTGGTCCTTGGTGTCAT-3'); Gammaglutamylcysteine synthetase ( $\gamma G C S$; sense: 5'-GCACATCTACCACGCCGTC-3' and antisense: $5^{\prime}$-CCACCTCATCGCCCCAC-3'). Beta-actin was used as a housekeeping gene ( $\beta$-actin; sense: 5'-CCTGGCACCCAGCACAAT-3' and antisense: 5'-GCCGATCCACACGGAGTACT-3'). The relative mRNA expression of HO-1 and $\gamma$ GCS was calculated according to the method of Livak and Schmittgen (39).

\subsection{Immunohistochemistry}

A549 cells were seeded in 4-chamber-slides (BD Falcon) at a density of 1600000 cells/well. The following day, cells were exposed to acrolein or the silver nanoparticles for $30 \mathrm{~min}$ or $4 \mathrm{~h}$, respectively. The control cells were treated in parallel for $4 \mathrm{~h}$ with FCScontaining phenol red free control medium. After gently washing with PBS buffer by means of three consecutive incubations for $5 \mathrm{~min}$ at $20{ }^{\circ} \mathrm{C}$ cells were fixed with $4 \%$ formaldehyde in PBS ( $\mathrm{pH} \mathrm{7.4)} \mathrm{for} 20 \mathrm{~min}$. The washing procedure was repeated and cells were then permeabilised with $0.1 \%$ Triton X-100 in PBS for 5 min. The cells were washed again and incubated with normal goat serum (1:65 dilution) for $30 \mathrm{~min}$. Cells were then washed and incubated overnight at $4{ }^{\circ} \mathrm{C}$ with a $1: 50$ diluted primary Nrf2 antibody (C-20, 
Santa Cruz Biotechnology, Inc.). Cells were washed again three times with PBS for $10 \mathrm{~min}$, followed by addition of AlexaFluor 594 secondary goat anti-rabbit IgG antibody (Life Technologies, Darmstadt, Germany) at 1:200 dilution and incubated for $1 \mathrm{~h}$ at room temperature. After performing the washing procedure again, cells were stained at room temperature with $1 \mathrm{\mu g} / \mathrm{ml}$ Hoechst 33342 nuclear dye dissolved in PBS for $15 \mathrm{~min}$. The washing procedure was repeated and cells were mounted with Prolong Gold Anti-fading (Life Technologies). Next day, images were acquired by Zeiss AxioVert Microscope equipped with an AxioCam MRm camera and analyzed using Zeiss (ZEN) software.

\subsection{Statistics}

All experiments were at least performed in duplicates at $\mathrm{n} \geq 3$ and all data are represented as mean \pm SEM. To test differences between the AgNP1 and AgNP2 groups a Two-Way Anova was used. Additionally, independent samples with equal variances were assessed for statistical significance with a t-test. P-values $<0.05$ were considered to be statistically significant.

\section{Results}

The cytotoxicity of both types of silver nanoparticles and acrolein towards the A549 lung epithelial cells was evaluated by the WST-1 assay. Results are shown in Figure 2 . A significant loss of metabolic activity could be observed with AgNP1 at the cell surface area treatment doses of 40 and $80 \mathrm{\mu g} / \mathrm{cm}^{2}$. In contrast, AgNP2 did not cause statistically significant toxicity up to the highest achievable treatment dose of $80 \mu \mathrm{g} / \mathrm{cm}^{2}$. The loss in viability was approximately $20 \%$ at this concentration. To enable a direct comparison of the toxic potency of the silver nanoparticles, in Figure $2 \mathrm{~B}$ the acrolein dose is also expressed in $\mu \mathrm{g} / \mathrm{cm}^{2}$, despite its non-(nano)particulate i.e. chemical nature. On this mass per cell surface area dose, the markedly higher toxicity of acrolein becomes apparent. The toxicity of acrolein towards the A549 cells reached statistical significance at concentrations of and above $10 \mu \mathrm{M}$, equivalent to $0.175 \mu \mathrm{g} / \mathrm{cm}^{2}$. Herein, it should be taken into account that the toxicity of acrolein was determined upon 30 min treatment, while for the two types of AgNP toxicity was evaluated after $24 \mathrm{~h}$. The calculated $\mathrm{TC}_{50}$ values for acrolein, AgNP1 and AgNP2 are $0.15 \pm 1.62 \mu \mathrm{g} / \mathrm{cm}^{2}, 55 \pm 12 \mu \mathrm{g} / \mathrm{cm}^{2}$ and $>80$ $\mu \mathrm{g} / \mathrm{cm}^{2}$ respectively. Based on the observed thresholds for cytotoxicity and the shape of the dose response curves, $10 \mu \mathrm{M}$ acrolein, $2.5 \mu \mathrm{g} / \mathrm{cm}^{2} \mathrm{AgNP} 1$ and $5 \mu \mathrm{g} / \mathrm{cm}^{2} \mathrm{AgNP2}$ were selected as pretreatment doses to evaluate potential adaptation mechanisms. As the toxic challenge concentrations, $100 \mu \mathrm{M}$ acrolein, $60 \mu \mathrm{g} / \mathrm{cm}^{2}$ AgNP1 and $80 \mu \mathrm{g} / \mathrm{cm}^{2}$ AgNP2 were chosen. 

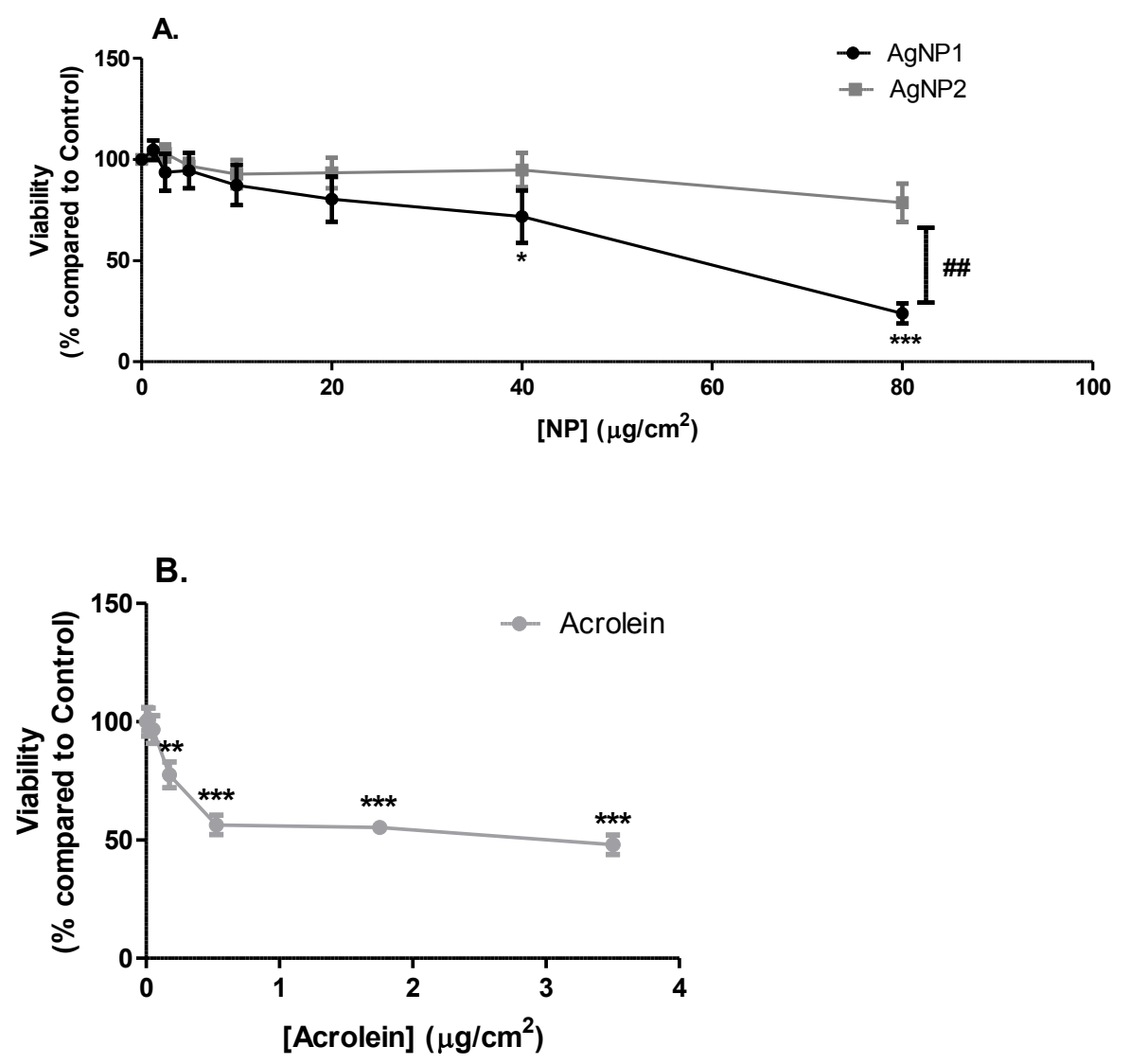

Figure 2: Effects of acrolein and AgNP1 and AgNP2 silver nanoparticles on cytotoxicity in A549 cells. The graphs shown in panel A (AgNP1 and AgNP2) and panel B (Acrolein) indicate mean \pm SEM from 4 independent experiments. ${ }^{*} \mathrm{P}<0.05 ;{ }^{*} \mathrm{P}<0.01 ;{ }^{* * * P}<$ 0.001 compared to control and \#P $<0.05$ compared to indicated condition.

To address hormesis of AgNPs, repeated exposure experiments were performed according to the time schemes shown in Supplementary Figure 1. A549 cells that were pretreated with $5 \mathrm{\mu g} / \mathrm{cm}^{2} \mathrm{AgNP2}$ for $24 \mathrm{~h}$ showed less toxicity than non-pretreated cells induced directly by a toxic challenge of $80 \mu \mathrm{g} / \mathrm{cm}^{2}$ of AgNP2 (Figure 3D) or $100 \mu \mathrm{M}$ acrolein (Figure 3E). Similarly, the cells that were pretreated with $2.5 \mathrm{\mu g} / \mathrm{cm}^{2} \mathrm{AgNP1}$ followed by a toxic challenge of $60 \mu \mathrm{g} / \mathrm{cm}^{2} \mathrm{AgNP} 1$ or $100 \mu \mathrm{M}$ acrolein showed an increasing trend in viability compared to the cells that were exposed to $60 \mu \mathrm{g} / \mathrm{cm}^{2}$ AgNP1 or $100 \mu \mathrm{M}$ acrolein without pretreatment (Figure $3 \mathrm{~B}$ and C). Finally, a significant 
diminishment of toxicity of $100 \mu \mathrm{M}$ acrolein was observed in response to pretreatment with $10 \mu \mathrm{M}$ acrolein (Figure 3A).
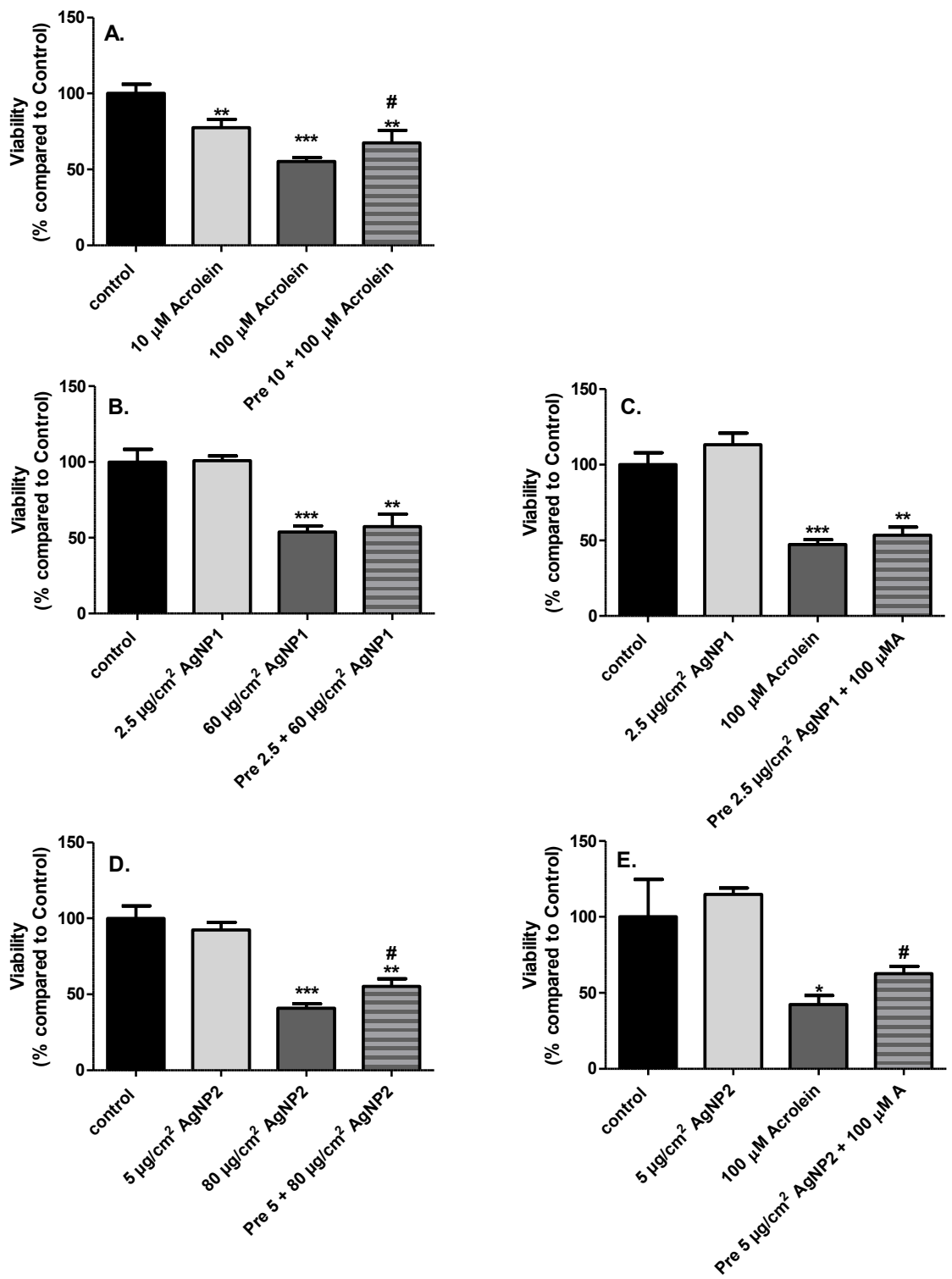

Figure 3: The effect of a pretreatment (=Pre) with $10 \mu \mathrm{M}$ Acrolein (A = Acrolein), 2.5 $\mu \mathrm{g} / \mathrm{cm}^{2}$ AgNP1 or $5 \mu \mathrm{g} / \mathrm{cm}^{2}$ AgNP2 on cell viability in A549 cells. $\mathrm{N}=4$ and data are presented as mean $\pm S E M$. The graphs indicate the effects of acrolein pretreatment on 
viability loss by acrolein (A), AgNP1 pretreatment on viability loss by AgNP1 (B), AgNP1 pretreatment on viability loss by acrolein (C), AgNP2 pretreatment on viability loss by AgNP2 (D), and the effect of AgNP2 pretreatment on viability loss by acrolein (E). *P < 0.05 ; * $\mathrm{P}<0.01 ; * * * \mathrm{P}<0.001$ compared to control \#P $<0.05$ compared to the respective non-pretreated cells.

The role of GSH in the toxicity and hormetic response was evaluated. The effects of the AgNPs and acrolein on GSH depletion in the A549 cells are shown in Figure 4. Both types of AgNPs caused a dose dependent decrease in intracellular GSH levels. A significant loss in GSH for AgNP1 was observed at $2.5 \mu \mathrm{g} / \mathrm{cm}^{2}$ and for AgNP2 at $20 \mu \mathrm{g} / \mathrm{cm}^{2}$. At the highest treatment concentrations $\left(80 \mu \mathrm{g} / \mathrm{cm}^{2}\right)$ the effect of AgNP1 was significantly stronger than the effect of the AgNP2 material. Acrolein treatment led to a significant decrease in intracellular GSH at $100 \mu \mathrm{M}$, equivalent to $1.18 \mu \mathrm{g} / \mathrm{cm}^{2}$ (Figure 4).

A.

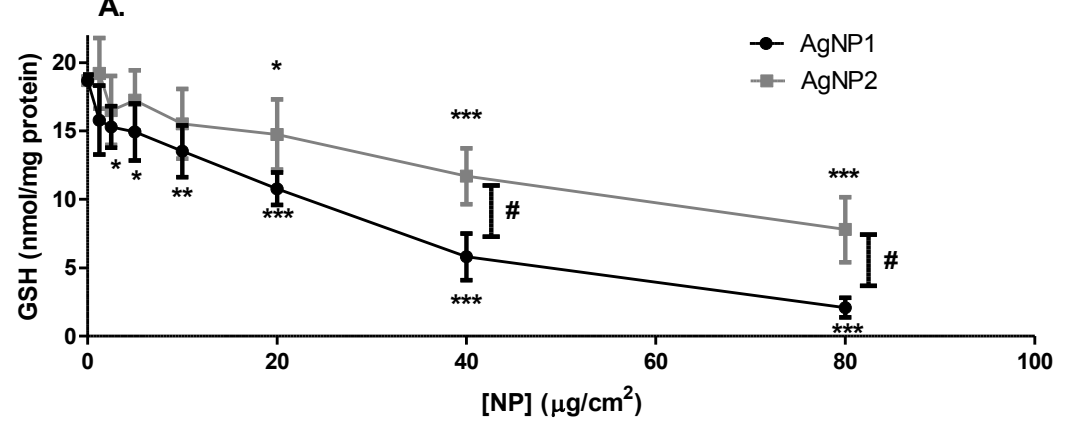

B.

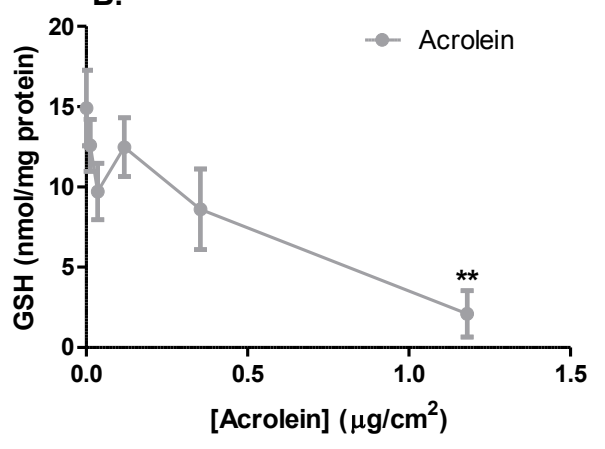

Figure 4: Effects of acrolein and AgNP1 and AgNP2 silver nanoparticles on intracellular GSH levels in A549 cells. The graphs shown in panel A (AgNP1 and AgNP2) and panel B (Acrolein) indicate mean \pm SEM from three independent experiments. ${ }^{*} \mathrm{P}<0.05 ; * * \mathrm{P}<$ 0.01 ; *** $\mathrm{P}<0.001$ compared to control and \#P $<0.05$ compared to indicated condition. 
Again, it should be noted that the GSH depletion of acrolein was measured upon 30 min treatment whereas the effects of the silver nanoparticles were evaluated following $24 \mathrm{~h}$ incubation. For acrolein as well as both types of AgNPs decreases in GSH levels were observed in association with viability loss. However, the extent of reduction in GSH in the A549 cells does not result in the same level of cytotoxicity for the three compounds studied. The concentrations at which a significant reduction in viability was seen did not correlate with the concentrations at which a significant decrease in GSH is observed (Supplementary Figure 2, Figure 2 and Figure 4).

Treatment of the A549 cells with acrolein at $100 \mu \mathrm{M}$ significantly diminished intracellular GSH levels. The pretreatment with $10 \mu \mathrm{M}$ acrolein prevented the significant loss of GSH after the subsequent toxic challenge at $100 \mu \mathrm{M}$ acrolein (Figure 5A). In contrast, pretreatment of the cells with AgNP1 $\left(2.5 \mu \mathrm{g} / \mathrm{cm}^{2}\right)$ or AgNP2 $\left(5 \mu \mathrm{g} / \mathrm{cm}^{2}\right)$ for $24 \mathrm{~h}$, followed by a challenge with $60 \mu \mathrm{g} / \mathrm{cm}^{2}$ AgNP1 or $80 \mu \mathrm{g} / \mathrm{cm}^{2}$ AgNP2 consecutively, did not prevent intracellular loss in GSH, when compared to the sole treatments with $60 \mu \mathrm{g} / \mathrm{cm}^{2}$ AgNP1 or $80 \mu \mathrm{g} / \mathrm{cm}^{2}$ AgNP2 respectively (Figure 5B and D). Pretreatment with AgNP1 or AgNP2 for $24 \mathrm{~h}$ also failed to protect the cells against the GSH depletion induced by acrolein (Figure 5C and E). The GSH levels in A549 cells were not changed following $24 \mathrm{~h}$ exposure to either $2.5 \mu \mathrm{g} / \mathrm{cm}^{2}$ AgNP1 or $5 \mu \mathrm{g} / \mathrm{cm}^{2}$ AgNP2 (Figure $5 \mathrm{~F}$ and G).

To further evaluate the potential underlying mechanisms of the observed effects, mRNA expression analyses and immunohistochemistry were performed. Treatment of the cells for $8 \mathrm{~h}$ with $10 \mu \mathrm{M}$ acrolein, $2.5 \mu \mathrm{g} / \mathrm{cm}^{2}$ AgNP1 and $5 \mu \mathrm{g} / \mathrm{cm}^{2}$ AgNP2 resulted in a significant increase in HO-1 expression (Figure 6). Moreover, acrolein also caused a significant increase in YGCS mRNA level. However, yGCS levels were not significantly changed in response to treatment with AgNP1 or AgNP2. Treatment of the A549 cells for $30 \mathrm{~min}$ with acrolein also increased in the immunoreactivity for Nrf2, indicative of the activation of this transcription factor. Representative staining results are shown in Figure 7. Digital analyses of revealed $19 \%$ increased red fluorescent signal compared to control cells ( $>400$ cells analysed/treatment). Enhanced immunoreactivity was also observed after $4 \mathrm{~h}$ treatment with AgNP1 $\left(2.5 \mu \mathrm{g} / \mathrm{cm}^{2}\right)$ and AgNP1 $\left(5 \mu \mathrm{g} / \mathrm{cm}^{2}\right)$ albeit to different extent, i.e. $30 \%$ and $17 \%$, respectively.

Figure 5: The effect of a pretreatment with $10 \mu \mathrm{M}$ Acrolein ( $A=A c r o l e i n)$, $2.5 \mu \mathrm{g} / \mathrm{cm}^{2}$ AgNP1 or $5 \mu \mathrm{g} / \mathrm{cm}^{2}$ AgNP2 on GSH levels in A549 cells. The graphs indicate the effects of acrolein pretreatment on intracellular GSH change by acrolein (A), AgNP1 pretreatment on GSH change by AgNP1 (B), AgNP1 pretreatment on GSH change by acrolein (C), AgNP2 pretreatment on GSH change by AgNP2 (D), AgNP2 pretreatment on GSH change by acrolein (E) and the effects of $2.5 \mu \mathrm{g} / \mathrm{cm}^{2}$ AgNP1 (F) or $5 \mu \mathrm{g} / \mathrm{cm}^{2}$ AgNP2 
(G) on GSH levels in A549 cells. $\mathbf{N} \geq 3$ and data are presented as mean \pm SEM. $* P<0.05$; $* * \mathrm{P}<0.01 ; * * * \mathrm{P}<0.001$ compared to control.
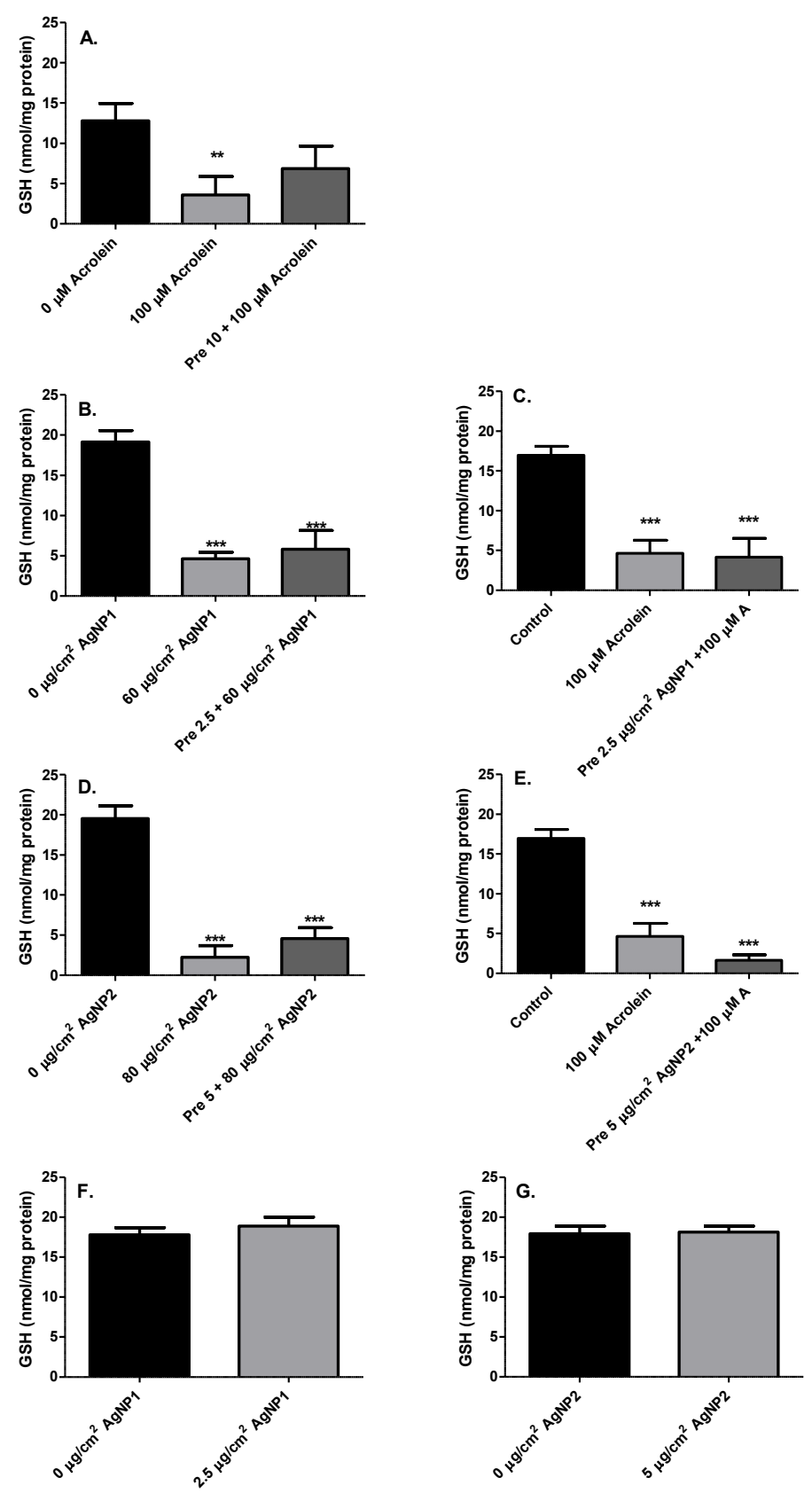

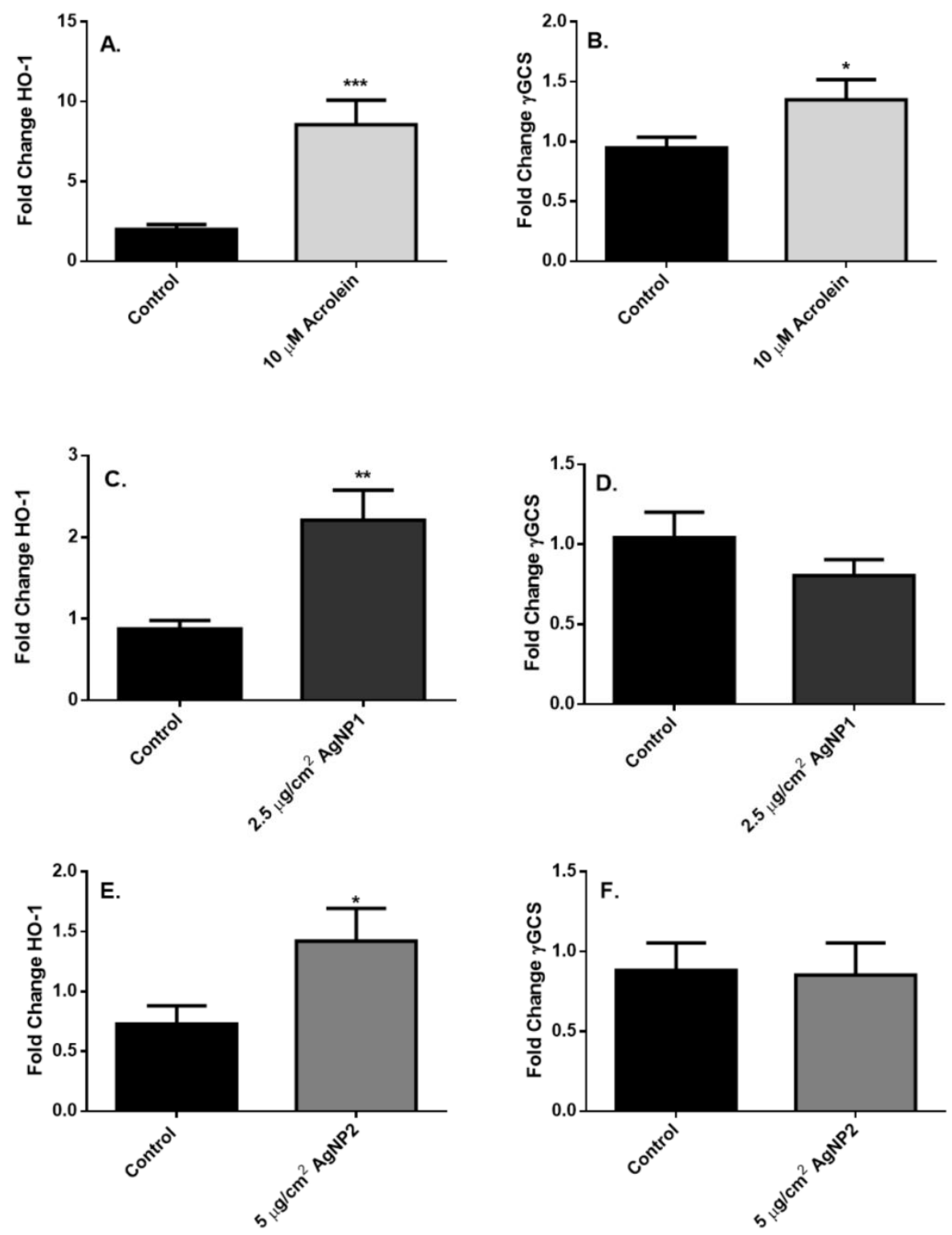

Figure 6: mRNA levels of Heme Oxygenase-1 (HO-1) (A, C and E) and gammaglutamylcysteine synthetase ( $\gamma$ GCS) (B, D and F) in A549 cells exposed to either $10 \mu \mathrm{M}$ Acrolein (A and B), $2.5 \mu \mathrm{g} / \mathrm{cm}^{2}$ (A and B), AgNP1 (C and D) or $5 \mu \mathrm{g} / \mathrm{cm}^{2}$ AgNP2 (E and F) for $8 \mathrm{~h}$. $\mathrm{N}=3$ and data are presented as mean \pm SEM. ${ }^{*} \mathrm{P}<0.05 ; * * \mathrm{P}<0.01 ; * * * \mathrm{P}<0.001$ compared to control. 


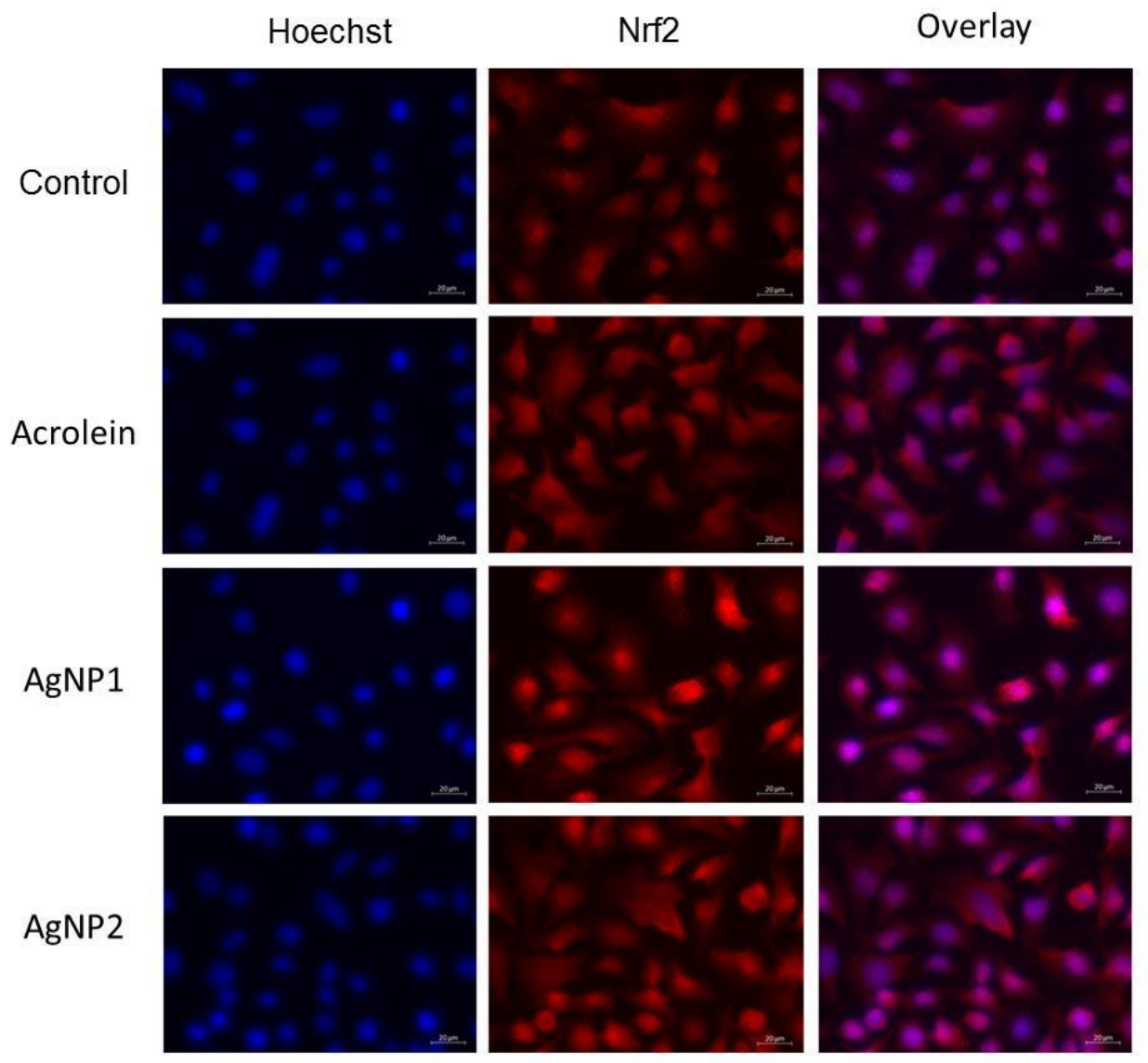

Figure 7: Immunohistochemical staining for Nrf2 in A549 cells after 4 h-treatment with $10 \mu \mathrm{M}$ acrolein, $2.5 \mu \mathrm{g} / \mathrm{cm}^{2}$ AgNP1 or $5 \mu \mathrm{g} / \mathrm{cm}^{2}$ AgNP2 at $400 \mathrm{X}$ magnification, in comparison to controls ( $4 \mathrm{~h}$-incubation in fresh test medium in the absence of AgNPs). The cells were stained for Nrf2 (red) while counterstaining with Hoechst 33342 is shown in blue.

\section{Discussion}

Increased application in medicine, consumer products including cosmetics, and even food underlines the need to examine thoroughly the toxicity of $\operatorname{AgNPs}(1,8,16)$. The present study provides evidence for hormesis of this type of nanomaterial, meaning that exposure to a low non-toxic dose of AgNPs might protect against a second toxic dose. Various physicochemical properties including particle size, surface area and dissolution rate have been linked to the toxicity of AgNPs e.g. an inverse correlation between their 
primary particle size and toxicity has been demonstrated in several studies $(14,32,40,41)$. In our hands, the toxicity of the AgNP2 sample $(16.6 \mathrm{~nm})$ was found to be markedly lower than that of the AgNP1 sample $(37.0 \mathrm{~nm})$. In line with this, the toxicities of the two samples were also inversely related to their solubility, as the AgNP2 sample showed a markedly higher dissolution in deionised water. Several studies have also shown that the toxicity of engineered nanoparticles, including AgNPs, relates to their ability to generate ROS $(1,17)$. However, using electron spin resonance spectroscopy $(42)$, results on radical formation for both AgNPs were less conclusive (data not shown).

Apart from the differences in primary particle size, solubility and reactivity, also contrasts in agglomeration/aggregation state and particle shape should be taken into account as well as the original state of the material, i.e. a powder versus a stabilized colloidal dispersion. DLS analyses revealed a better dispersion state of the AgNP2 sample in water. However, in the FCS-containing culture medium, which reflects cell treatment conditions, the Z-average and PDI increased for this sample. Comparative analysis in FCSfree culture medium indicated the impact of the $10 \%$ FCS on agglomeration behavior for this colloidal sample. In contrast, the AgNP1 had a larger hydrodynamic size and polydispersity, irrespective of the suspension conditions. To unravel the mechanism of the hormetic response, acrolein was also investigated in this study. Both AgNPs appeared to be less toxic than acrolein at equal dose $\left(\mu \mathrm{g} / \mathrm{cm}^{2}\right)$, and may relate to different cellular uptake processes and kinetics (43). AgNPs are taken up and compartmentalized in the cells, primarily by endocytosis and through several metal transporters or ATPases, whereas acrolein uptake probably mainly involves rapid, passive diffusion through the lipid membrane $(30,44)$.

Previous research in BEAS-2B bronchial epithelial cells showed that GSH is the first line of defense against acrolein $(20,28)$. In the present study with $A 549$ alveolar epithelial cells, acrolein toxicity is also accompanied by a decrease in GSH. The BEAS-2B cells seem to be less vulnerable than the A549 cells, because more acrolein is needed to induce the same level of toxicity. This difference in toxicity has also been seen for other substances including multi-walled carbon nanotubes (MWCNTs) that are more toxic in BEAS-2B cells than in A549 cells (45). This difference in toxicity can be explained by differences in antioxidant defense systems between the different cell types (46). One of these antioxidant defense mechanisms is the GSH system. Remarkably, which further underlines the role of GSH as protector against toxicity is that in this study in A549 cells an increase in viability is correlated with less decrease in GSH. In BEAS-2B cells the GSH level dropped at lower levels of acrolein compared to A549 cells. This corresponds to relating the vulnerability to toxic compounds of A549 cells to BEAS-2B cells (47). For AgNP2 and AgNP1, an increase in viability is also associated with an increase in GSH. Such an effect for AgNPs has for instance also been reported in Caco-2 intestinal epithelial cells $(48,49)$, HepG2 liver epithelial cells (50), and HK-2 kidney epithelial cells (18). The importance of 112 
GSH in the protection against AgNP toxicity is supported by the protective effect of the glutathione precursor $\mathrm{N}$-acetylcysteine $(51,52)$. Apart from the protection provided by $\mathrm{GSH}$, cells also have alternative pathways to protect against toxicity of AgNPs (53). For example, in A549 cells more Nrf2-mediated upregulation of the endogenous antioxidant response has been observed (54).

In the present study, it was found that acrolein, such as in BEAS-2B cells (28), also induces hormesis in A549 alveolar epithelial cells. GSH functions as the first line of defense against acrolein. Then, Keap1 is adducted by acrolein (55) and Nrf2-mediated gene expression of the antioxidant enzyme HO-1 and the rate-limiting enzyme of GSH synthesis YGCS are induced. The last one probably contributes to the higher GSH level in pretreated A549 cells and explains why pretreated A549 cells show less cytotoxicity in case of pretreatment with acrolein. In case of the AgNPs, pretreatment with a low dose of AgNP1 or AgNP2 before a treatment with a toxic dose of these respective samples did show less cytotoxicity than without pretreatment, also indicating hormesis. Notably, however, the effect was stronger and only statistically significant for AgNP2, i.e. the colloidal sample with the smaller primary particle size and associated higher solubility, and the relatively lower agglomeration behavior in the testing medium. This indicates that adaptation depends on the specific physicochemical properties of this type of nanomaterial.

A major finding in our study was that the pretreatment of the A549 cells with a low dose of the silver nanoparticles also offered protection against the toxic effects of a treatment with a toxic concentration of $100 \mu \mathrm{M}$ acrolein. Thus, in this case, a low dose of a substance that is damaging at high doses also protects against the damaging effects of another toxic substance at its toxic concentration. Therefore, we would like to introduce the term transhormesis, hormesis induced by a different chemical than the chemical of exposure. This finding would implicate that both acrolein and nanosilver, depending on its dose and (physico-)chemical properties can induce adaptation by inducing a similar protective mechanism. Only then, exposure to the low dose of one substance could induce protection for a toxic dose of another chemical. Since GSH has been shown to be the first line of defense against acrolein in BEAS-2B cells, we expected this to be the mechanism by which silver nanoparticles upregulate cellular protection. However, only for acrolein it could be confirmed that GSH plays a role as a protective mechanism in response to low dose pretreatment of this compound: The GSH level was significantly higher in A549 cells exposed to $100 \mu \mathrm{M}$ of acrolein preceded by a pretreatment with $10 \mu \mathrm{M}$ of acrolein compared to $\mathrm{A} 549$ cells that were exposed to $100 \mu \mathrm{M}$ of acrolein without pretreatment. However, for neither of the AgNPs, such significant difference could be observed. From this it can be concluded that GSH is not the key in the adaptation induced by the silver nanoparticles. Moreover, our findings also indicate that there has to exist another 
protective mechanism that is induced by nanosilver to protect the cells from the toxic concentration of acrolein.

Immunohistochemical analyses indicated activation of the cellular redox sensor Nrf2 in the A549 cells by acrolein as well as the AgNPs, which is in concordance with other investigations $(16,18,20,21,56)$. Remarkably, both $y$ GCS and HO-1 were found to been upregulated by acrolein and AgNPs only increase $\mathrm{HO}-1$, whereas both genes are regulated by $\operatorname{Nrf2}(16,18,19)$. Timing could play an important role, because for adaptation induced by acrolein only $4 \mathrm{~h}$ are required, while for AgNPs adaptation is induced over a time period of $24 \mathrm{~h}(24,27)$. Besides timing also other factors including the specific physicochemical properties, cellular uptake and dissolution of the applied nanoparticles or the cell lines used could be involved, which can explain why acrolein showed enhanced mRNA expression for $\mathrm{GGCS}$ as well as for HO-1, while AgNPs only increase HO-1. Kang and coworkers (19) could demonstrate that blocking of Nrf2 resulted in increased DNA damage and apoptosis by AgNPs in ovarian carcinoma cells. Moreover, they showed that HO-1 induction by cobalt protoporphyrin offered protection against AgNP-induced toxicity, whereas specific inhibition of HO-1 exacerbated the effects of the nanoparticles. This would be in support of the role of the HO-1 protein in the observed adaptation by nanosilver in the A549 cells. Interestingly, in this regard, these effects shown by Kang and coworkers, occurred in association with AgNP-mediated activation of the PI3K and p38MAPK signalling pathways. In HBE1 human bronchial epithelial cells a role for protein kinase $\mathrm{C}$ delta (PKC-delta) in Nrf2-mediated induction of HO-1 by acrolein was previously shown by Zhang and Forman (56). Thus, HO-1 could also be a main player in the observed transhormesis effect by AgNPs and acrolein, although a direct protective effect of $\mathrm{HO}-1$ on acrolein in the $\mathrm{A} 549$ cells remains to be investigated.

Potential adaptation mechanisms to the toxicity of AgNPs have been discussed in a number of recent studies $(23,24,57)$. Comparing the effects of a single high versus repeated low doses of AgNPs, Aude-Garcia and colleagues revealed the importance of addressing the dose rate in the toxicity of nanoparticles. Although adaptation was not in the focus of their work, it would be interesting to investigate to what extent changes in dose rate would affect the observed effects in our study. In line with this, it would also be of great relevance to further investigate the role of specific physicochemical properties, target cell-specific interactions on adaptation and further examine the time dependency of the adaptation. It should be underlined that GSH plays a role in short term adaptation, whereas Nrf2-mediated mechanisms provides protection on a longer term, which hypothetically confirms our findings (58), but should still be verified experimentally. Acrolein is taken up rapidly by passive diffusion, whereas silver nanoparticles are taken up by endocytosis and then subject to intracellular dissolution $(30,44)$ which agrees more with a long term exposure. 
In conclusion, AgNPs can induce hormetic adaptation. GSH is related to toxicity but not to hormesis, which is in contrast to findings with acrolein in which GSH is linked to both toxicity and hormesis. These findings have implication with regard to the use of AgNPs in medicine as well as to risk assessment, because consumers are repeatedly exposed to a relatively high concentration of AgNPs. The precise molecular mechanism of hormesis by AgNPs is still enigmatic, but Nrf2 mediated signaling seems to be involved. In view of the established importance of oxidative stress and redox-signaling in the toxicity of many nanomaterials it will be interesting to investigate whether other types of nanoparticles also can induce hormetic adaptation via a similar mechanism as AgNPs.

\section{Supplement}

\subsection{Supplementary Material and Methods}

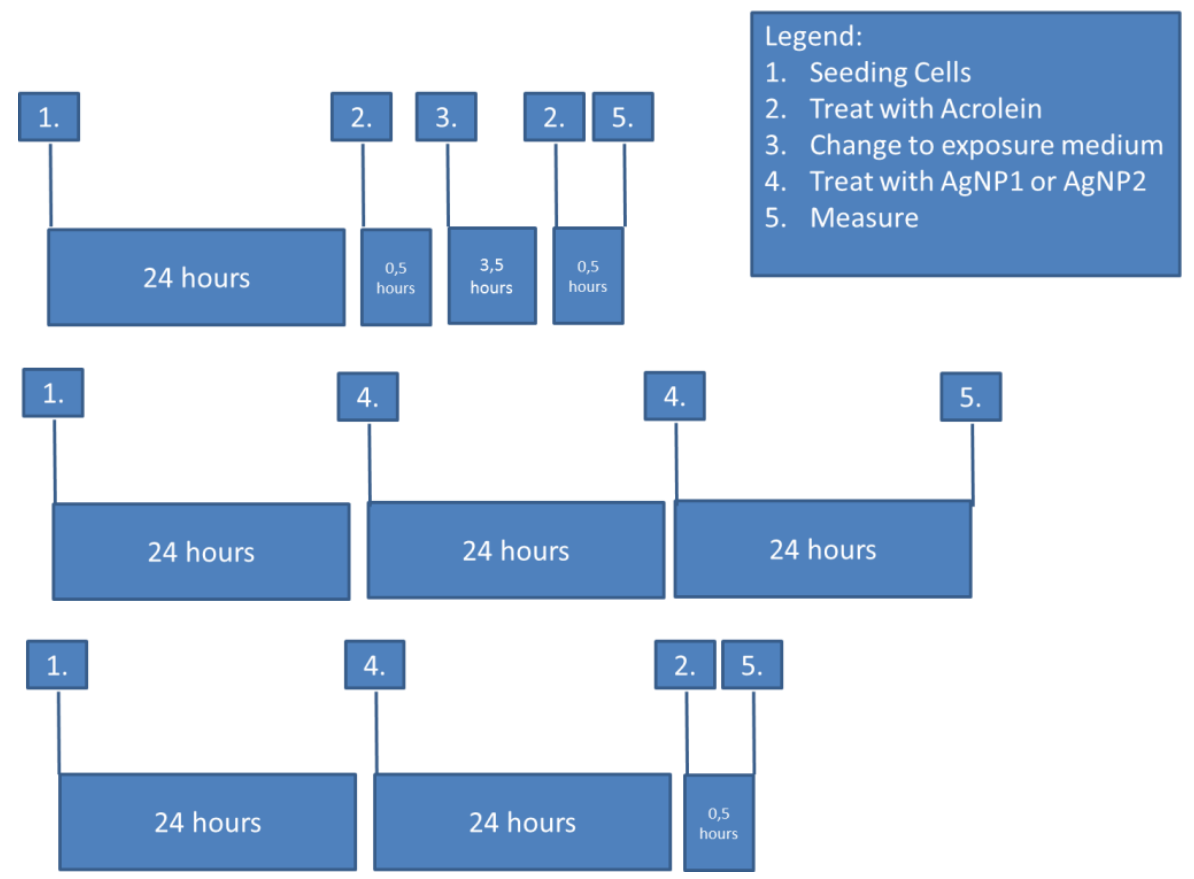

Supplementary Figure 1: Timeline for treatments to investigate the adaptation principle in A549 cells. 


\subsection{Supplementary Results}
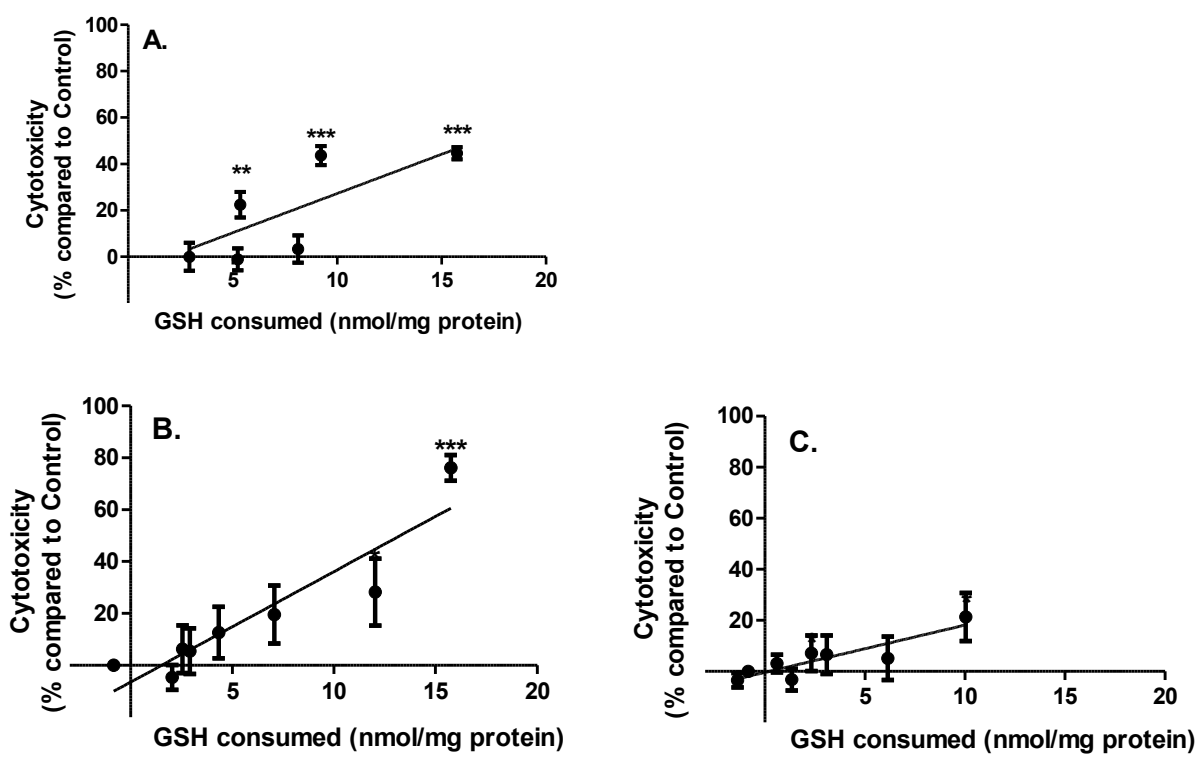

Supplementary Figure 2: Association of cytotoxicity and the amount of GSH consumed in A549 cells exposed to Acrolein (A), AgNP1 (B) and AgNP2 (C). N=3 and data are presented as mean \pm SEM. $* \mathrm{P}<0.05 ; * \mathrm{P}<0.01 ; * * * \mathrm{P}<0.001$ compared to control. 


\section{References}

1. Johnston HJ, Hutchison G, Christensen FM, Peters S, Hankin S, Stone V. A review of the in vivo and in vitro toxicity of silver and gold particulates: particle attributes and biological mechanisms responsible for the observed toxicity. Critical reviews in toxicology. $2010 \mathrm{Apr} ; 40(4): 328-46$.

2. Austin LA, Mackey MA, Dreaden EC, El-Sayed MA. The optical, photothermal, and facile surface chemical properties of gold and silver nanoparticles in biodiagnostics, therapy, and drug delivery. Archives of toxicology. 2014 Jul;88(7):1391-417.

3. Batchelor-McAuley CT, K; Neumann, C.C.M.; Laborda, E; Compton, R.G. Why are Silver Nanoparticles More Toxic Than Bulk Silver? Towards Understanding the Dissolution and Toxicity of Silver Nanoparticles. International Journal of ELECTROCHEMICAL SCIENCE. 2014;9:1132 - 8.

4. Levard C, Hotze EM, Colman BP, Dale AL, Truong L, Yang XY, et al. Sulfidation of silver nanoparticles: natural antidote to their toxicity. Environ Sci Technol. 2013 Dec 3;47(23):13440-8.

5. Cronholm P, Karlsson HL, Hedberg J, Lowe TA, Winnberg L, Elihn K, et al. Intracellular uptake and toxicity of $\mathrm{Ag}$ and $\mathrm{CuO}$ nanoparticles: a comparison between nanoparticles and their corresponding metal ions. Small. $2013 \mathrm{Apr}$ 8;9(7):970-82.

6. Beer C, Foldbjerg R, Hayashi Y, Sutherland DS, Autrup H. Toxicity of silver nanoparticles - nanoparticle or silver ion? Toxicol Lett. 2012 Feb 5;208(3):286-92.

7. Govender R, Phulukdaree A, Gengan RM, Anand K, Chuturgoon AA. Silver nanoparticles of Albizia adianthifolia: the induction of apoptosis in human lung carcinoma cell line. J Nanobiotechnology. 2013;11:5.

8. McShan D, Ray PC, Yu H. Molecular toxicity mechanism of nanosilver. J Food Drug Anal. 2014 Mar;22(1):116-27.

9. Braakhuis HM, Gosens I, Krystek P, Boere JA, Cassee FR, Fokkens PH, et al. Particle size dependent deposition and pulmonary inflammation after short-term inhalation of silver nanoparticles. Part Fibre Toxicol. 2014;11:49.

10. De Matteis V, Malvindi MA, Galeone A, Brunetti V, De Luca E, Kote S, et al. Negligible particle-specific toxicity mechanism of silver nanoparticles: the role of Ag+ ion release in the cytosol. Nanomedicine. 2015 Apr;11(3):731-9. 
11. Recordati C, De Maglie M, Bianchessi S, Argentiere S, Cella C, Mattiello S, et al. Tissue distribution and acute toxicity of silver after single intravenous administration in mice: nano-specific and size-dependent effects. Part Fibre Toxicol. 2016;13:12.

12. Donaldson K, Stone V, Borm PJ, Jimenez LA, Gilmour PS, Schins RP, et al. Oxidative stress and calcium signaling in the adverse effects of environmental particles (PM10). Free radical biology \& medicine. 2003 Jun 1;34(11):1369-82.

13. Unfried K, Albrecht C, Klotz LO, von Mikecz A, Grether-Beck S, R.P.F. S. Cellular responses to nanoparticles: target structures and mechanisms. Nanotoxicology 2007;1:52-71.

14. Liu W, Wu Y, Wang C, Li HC, Wang T, Liao CY, et al. Impact of silver nanoparticles on human cells: effect of particle size. Nanotoxicology. 2010 Sep;4(3):319-30.

15. Foldbjerg R, Irving ES, Hayashi Y, Sutherland DS, Thorsen K, Autrup H, et al. Global gene expression profiling of human lung epithelial cells after exposure to nanosilver. Toxicological sciences : an official journal of the Society of Toxicology. 2012 Nov;130(1):145-57.

16. Bohmert L, Niemann B, Lichtenstein D, Juling S, Lampen A. Molecular mechanism of silver nanoparticles in human intestinal cells. Nanotoxicology. 2015 May 21:1-9.

17. Xia T, Kovochich M, Brant J, Hotze M, Sempf J, Oberley $T$, et al. Comparison of the abilities of ambient and manufactured nanoparticles to induce cellular toxicity according to an oxidative stress paradigm. Nano letters. 2006 Aug;6(8):1794-807.

18. Kang SJ, Lee YJ, Lee EK, Kwak MK. Silver nanoparticles-mediated G2/M cycle arrest of renal epithelial cells is associated with NRF2-GSH signaling. Toxicol Lett. 2012 Jun 20;211(3):334-41.

19. Kang SJ, Ryoo IG, Lee YJ, Kwak MK. Role of the Nrf2-heme oxygenase-1 pathway in silver nanoparticle-mediated cytotoxicity. Toxicology and applied pharmacology. 2012 Jan 1;258(1):89-98.

20. Prasad RY, McGee JK, Killius MG, Suarez DA, Blackman CF, DeMarini DM, et al. Investigating oxidative stress and inflammatory responses elicited by silver nanoparticles using high-throughput reporter genes in HepG2 cells: effect of size, surface coating, and intracellular uptake. Toxicology in vitro : an international journal published in association with BIBRA. 2013 Sep;27(6):2013-21. 
21. Sahu SC, Zheng J, Yourick JJ, Sprando RL, Gao X. Toxicogenomic responses of human liver HepG2 cells to silver nanoparticles. J Appl Toxicol. 2015 Oct;35(10):1160-8.

22. Zhang H, Liu H, Davies KJ, Sioutas C, Finch CE, Morgan TE, et al. Nrf2regulated phase II enzymes are induced by chronic ambient nanoparticle exposure in young mice with age-related impairments. Free radical biology \& medicine. 2012 May 1;52(9):2038-46.

23. Aude-Garcia C, Villiers F, Collin-Faure V, Pernet-Gallay K, Jouneau PH, Sorieul $\mathrm{S}$, et al. Different in vitro exposure regimens of murine primary macrophages to silver nanoparticles induce different fates of nanoparticles and different toxicological and functional consequences. Nanotoxicology. 2015 Nov 10:1-11.

24. Brzoska K, Meczynska-Wielgosz S, Stepkowski TM, Kruszewski M. Adaptation of HepG2 cells to silver nanoparticles-induced stress is based on the pro-proliferative and anti-apoptotic changes in gene expression. Mutagenesis. 2015 May;30(3):431-9.

25. Calabrese EJ, Baldwin LA. Defining hormesis. Hum Exp Toxicol. 2002 Feb;21(2):91-7.

26. lavicoli I, Calabrese EJ, Nascarella MA. Exposure to nanoparticles and hormesis. Dose Response. 2010;8(4):501-17.

27. Bell IR, Ives JA, Jonas WB. Nonlinear effects of nanoparticles: biological variability from hormetic doses, small particle sizes, and dynamic adaptive interactions. Dose Response. 2014 May;12(2):202-32.

28. Sthijns MM, Randall MJ, Bast A, Haenen GR. Adaptation to acrolein through upregulating the protection by glutathione in human bronchial epithelial cells: the materialization of the hormesis concept. Biochem Biophys Res Commun. 2014 Apr 18;446(4):1029-34.

29. Haenen GR, Vermeulen NP, Tai Tin Tsoi JN, Ragetli HM, Timmerman H, Blast A. Activation of the microsomal glutathione-S-transferase and reduction of the glutathione dependent protection against lipid peroxidation by acrolein. Biochemical pharmacology. 1988 May 15;37(10):1933-8.

30. Stevens JF, Maier CS. Acrolein: sources, metabolism, and biomolecular interactions relevant to human health and disease. Mol Nutr Food Res. 2008 Jan;52(1):7-25. 
31. Singh S, Shi T, Duffin R, Albrecht $C$, van Berlo D, Hohr D, et al. Endocytosis, oxidative stress and IL-8 expression in human lung epithelial cells upon treatment with fine and ultrafine TiO2: role of the specific surface area and of surface methylation of the particles. Toxicology and applied pharmacology. $2007 \mathrm{Jul}$ 15;222(2):141-51.

32. Maurer EI, Sharma M, Schlager JJ, Hussain SM. Systematic analysis of silver nanoparticle ionic dissolution by tangential flow filtration: toxicological implications. Nanotoxicology. 2014 Nov;8(7):718-27.

33. Huk A, Izak-Nau E, Reidy B, Boyles M, Duschl A, Lynch I, et al. Is the toxic potential of nanosilver dependent on its size? Part Fibre Toxicol. 2014;11:65.

34. Han JW, Gurunathan S, Jeong JK, Choi YJ, Kwon DN, Park JK, et al. Oxidative stress mediated cytotoxicity of biologically synthesized silver nanoparticles in human lung epithelial adenocarcinoma cell line. Nanoscale Res Lett. 2014;9(1):459.

35. Christensen FM, Johnston HJ, Stone V, Aitken RJ, Hankin S, Peters S, et al. Nano-silver - feasibility and challenges for human health risk assessment based on open literature. Nanotoxicology. 2010 Sep;4(3):284-95.

36. Kermanizadeh A, Gosens I, MacCalman L, Johnston H, Danielsen PH, Jacobsen NR, et al. A Multilaboratory Toxicological Assessment of a Panel of 10 Engineered Nanomaterials to Human Health-ENPRA Project-The Highlights, Limitations, and Current and Future Challenges. J Toxicol Environ Health B Crit Rev. 2016;19(1):1-28.

37. Kermanizadeh A, Vranic S, Boland S, Moreau K, Baeza-Squiban A, Gaiser $B K$, et al. An in vitro assessment of panel of engineered nanomaterials using a human renal cell line: cytotoxicity, pro-inflammatory response, oxidative stress and genotoxicity. BMC Nephrol. 2013;14:96.

38. Rahman I, Kode A, Biswas SK. Assay for quantitative determination of glutathione and glutathione disulfide levels using enzymatic recycling method. Nat Protoc. 2006;1(6):3159-65.

39. Livak KJ, Schmittgen TD. Analysis of relative gene expression data using real-time quantitative PCR and the 2(-Delta Delta C(T)) Method. Methods. 2001 Dec;25(4):402-8.

40. Wildt BE, Celedon A, Maurer El, Casey BJ, Nagy AM, Hussain SM, et al. Intracellular accumulation and dissolution of silver nanoparticles in L-929 
fibroblast cells using live cell time-lapse microscopy. Nanotoxicology. 2015 Dec 8:1-10.

41. Mishra A, Zheng J, Tang X, Goering PL. Silver Nanoparticle-Induced Autophagic-Lysosomal Disruption and NLRP3-Inflammasome Activation in HepG2 Cells is Size-Dependent. Toxicological sciences : an official journal of the Society of Toxicology. 2016 Jan 21.

42. Papageorgiou I, Brown C, Schins R, Singh S, Newson R, Davis S, et al. The effect of nano- and micron-sized particles of cobalt-chromium alloy on human fibroblasts in vitro. Biomaterials. $2007 \mathrm{Jul} ; 28(19): 2946-58$.

43. Gliga AR, Skoglund S, Wallinder IO, Fadeel B, Karlsson HL. Size-dependent cytotoxicity of silver nanoparticles in human lung cells: the role of cellular uptake, agglomeration and Ag release. Part Fibre Toxicol. 2014;11:11.

44. Behra R, Sigg L, Clift MJ, Herzog F, Minghetti M, Johnston B, et al. Bioavailability of silver nanoparticles and ions: from a chemical and biochemical perspective. J R Soc Interface. 2013 Oct 6;10(87):20130396.

45. Ursini CL, Cavallo D, Fresegna AM, Ciervo A, Maiello R, Buresti G, et al. Differences in cytotoxic, genotoxic, and inflammatory response of bronchial and alveolar human lung epithelial cells to pristine and $\mathrm{COOH}$-functionalized multiwalled carbon nanotubes. BioMed research international. 2014;2014:359506. 46. Zhang $\mathrm{H}$, Wang X, Wang $\mathrm{M}$, Li L, Chang CH, Ji Z, et al. Mammalian Cells Exhibit a Range of Sensitivities to Silver Nanoparticles that are Partially Explicable by Variations in Antioxidant Defense and Metallothionein Expression. Small. 2015 Aug;11(31):3797-805.

47. Schlinkert $P$, Casals $E$, Boyles $M$, Tischler $U$, Hornig E, Tran N, et al. The oxidative potential of differently charged silver and gold nanoparticles on three human lung epithelial cell types. J Nanobiotechnology. 2015;13:1.

48. Aueviriyavit $S$, Phummiratch D, Maniratanachote R. Mechanistic study on the biological effects of silver and gold nanoparticles in Caco-2 cells--induction of the Nrf2/HO-1 pathway by high concentrations of silver nanoparticles. Toxicology letters. 2014 Jan 3;224(1):73-83.

49. McCracken C, Zane A, Knight DA, Hommel E, Dutta PK, Waldman WJ. Oxidative stress-mediated inhibition of intestinal epithelial cell proliferation by silver nanoparticles. Toxicology in vitro : an international journal published in association with BIBRA. 2015 Oct;29(7):1793-808. 
50. Vrcek IV, Zuntar I, Petlevski R, Pavicic I, Dutour Sikiric M, Curlin M, et al. Comparison of in vitro toxicity of silver ions and silver nanoparticles on human hepatoma cells. Environ Toxicol. 2014 Nov 28.

51. Foldbjerg R, Dang DA, Autrup H. Cytotoxicity and genotoxicity of silver nanoparticles in the human lung cancer cell line, A549. Archives of toxicology. $2011 \mathrm{Jul} ; 85(7): 743-50$.

52. George S, Lin S, Ji Z, Thomas CR, Li L, Mecklenburg M, et al. Surface defects on plate-shaped silver nanoparticles contribute to its hazard potential in a fish gill cell line and zebrafish embryos. ACS Nano. 2012 May 22;6(5):3745-59.

53. Chairuangkitti $P$, Lawanprasert $S$, Roytrakul $S$, Aueviriyavit $S$, Phummiratch $D$, Kulthong $K$, et al. Silver nanoparticles induce toxicity in A549 cells via ROSdependent and ROS-independent pathways. Toxicology in vitro : an international journal published in association with BIBRA. $2013 \mathrm{Feb}$;27(1):330-8.

54. Singh A, Misra V, Thimmulappa RK, Lee H, Ames S, Hoque MO, et al. Dysfunctional KEAP1-NRF2 interaction in non-small-cell lung cancer. PLoS Med. 2006 Oct;3(10):e420.

55. McMahon M, Lamont DJ, Beattie KA, Hayes JD. Keap1 perceives stress via three sensors for the endogenous signaling molecules nitric oxide, zinc, and alkenals. Proc Natl Acad Sci U S A. 2010 Nov 2;107(44):18838-43.

56. Zhang $\mathrm{H}$, Forman $\mathrm{HJ}$. Acrolein induces heme oxygenase-1 through PKCdelta and PI3K in human bronchial epithelial cells. Am J Respir Cell Mol Biol. 2008 Apr;38(4):483-90.

57. Jiao ZH, Li M, Feng YX, Shi JC, Zhang J, Shao B. Hormesis effects of silver nanoparticles at non-cytotoxic doses to human hepatoma cells. PLoS One. 2014;9(7):e102564.

58. Sthijns MM, Weseler AR, Bast A, Haenen GR. Time in Redox Adaptation Processes: From Evolution to Hormesis. International journal of molecular sciences. 2016 Sep 29;17(10). 


Chapter 5

Rutin protects against $\mathrm{H}_{2} \mathrm{O}_{2}$-triggered impaired relaxation of placental arterioles and induces Nrf2-mediated adaptation in Human Umbilical Vein Endothelial Cells exposed to oxidative stress.

Mireille M.J.P.E. Sthijns, Paul M. Schiffers, Ger M. Janssen, Kristien J.A. Lemmens, Bart Ides, Philippe Vangrieken, Freek G. Bouwman, Edwin C. Mariman, Irina Pader, Elias S.J. Arnér, Katarina Johansson, Aalt Bast, Guido R.M.M. Haenen.

Biochimica Biophysica Acta - General Subjects. 2017 Mar 7;1861(5 Pt A):1177-1189. 


\section{Abstract}

\subsection{Background}

Rutin intake is associated with a reduced risk of cardiovascular disease (CVD). The exact mechanism by which rutin can protect against CVD development is still enigmatic. Since, rutin is a compound with a relatively short half-life, the direct antioxidant effect of rutin can not explain the long-lasting effect on human health. We hypothesized that rutin next to its direct antioxidant that improves endothelial function, may also induce an adaptive response in endogenous antioxidant systems.

\subsection{Methods and results}

In Human Umbilical Vein Endothelial Cells (HUVECs), the direct antioxidant effect was confirmed. During scavenging of Reactive Oxygen Species (ROS), rutin is oxidized into a quinone derivative. HUVECs pretreated with rutin quinone became better protected against a second challenge with oxidative stress 3 hours later. LC-MS/MS analysis indicated that rutin quinone targets cysteine 151 of Keap1. Moreover, we found that the quinone is an inhibitor of the selenoprotein thioredoxin reductase 1 . These properties correlated with an activation of Nrf2 and upregulation of Glutamate Cysteine Ligase, the rate-limiting enzyme of glutathione synthesis, while NF-KB and HIF activation became blunted by rutin treatment. Furthermore, rutin was found to prevent hydrogen peroxide from impairing relaxation of human chorionic plate placental vessels, which may help to protect endothelial function.

\subsection{Conclusion and significance}

Rutin functions as an antioxidant and is oxidized into a quinone that upregulates the Nrf2-mediated endogenous antioxidant response. This mechanism suggests that rutin selectively exerts its protective effects in regions with increased oxidative stress, and explains how rutin reduces the risk of developing CVD.

\subsection{General significance}

The newly found mechanism behind the long term protection of rutin against cardiovascular disease, the selective upregulation of endogenous antioxidant systems, contribute to the further understanding why rutin can reduce the risk on developing cardiovascular disease.

Trial registration: Netherlands Trial Register: METC number: 13-4-102. 


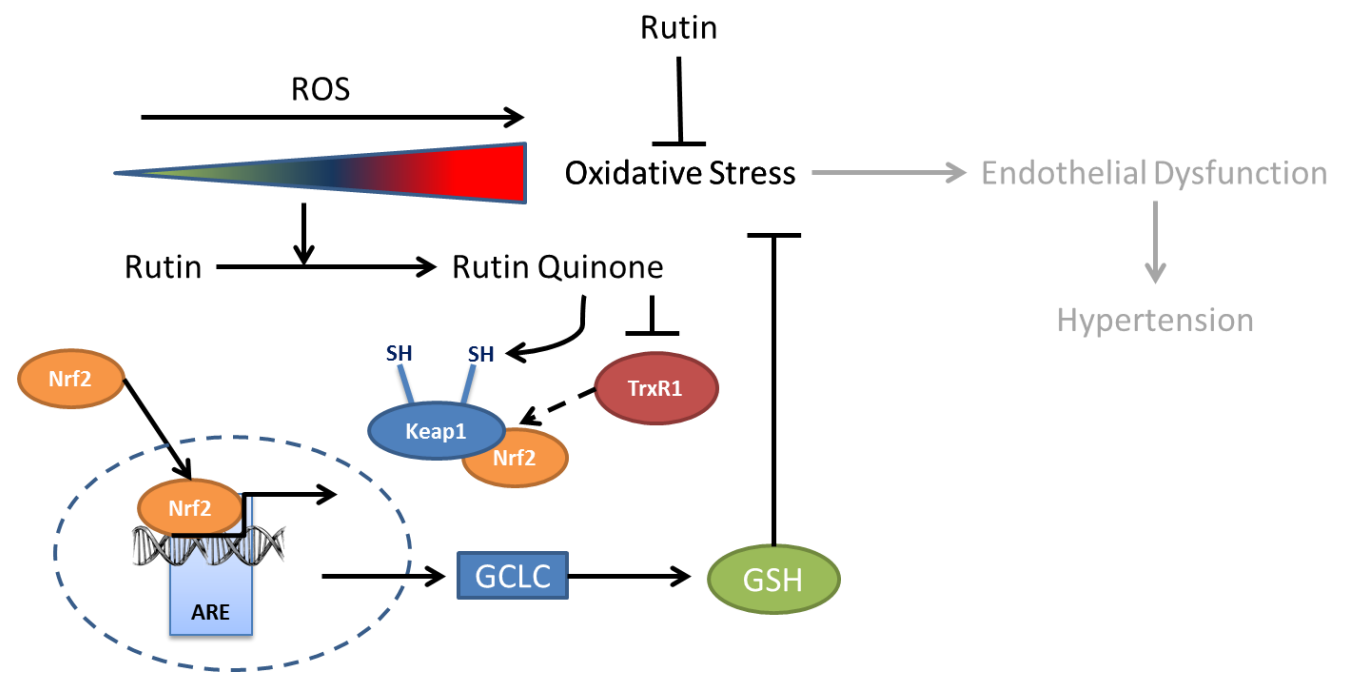




\section{Introduction}

Rutin is an antioxidant flavonoid abundantly present in the human diet. The average daily intake of rutin via food is $5-10 \mathrm{mg} /$ day that results in blood plasma concentrations of $0.20 \pm 0.06 \mu \mathrm{M}$ (1). With supplementation even higher concentrations can be achieved (2). Rats supplemented with $150 \mathrm{mg}$ of rutin, had a plasma concentration of $20 \mu \mathrm{M}$ after 1 hour, whereas after 24 hours approximately $35 \mu \mathrm{M}$ can be measured (3). In humans, supplementation of $100 \mathrm{mg}$ of rutin resulted approximately in a concentration of $0.2 \mu \mathrm{M}$ determined in the blood plasma (4). And supplementation with $1000 \mathrm{mg}$ of a flavonoid a day has been performed (5). High amounts of rutin are found in buckwheat and citrus fruits (6). Rutin intake is associated with a reduced risk of cardiovascular disease (CVD) (7). Additionally, intake of rutosides appears to have a protective effect both in the treatment and prevention of post-thrombotic syndrome (8). However, the exact mechanism by which rutin can prevent CVD development is still unknown. The major risk factor for developing CVD is hypertension, causing almost $50 \%$ of ischemic strokes and increases the risk of hemorrhagic stroke (9). In hypertension oxidative stress mediated endothelial dysfunction is frequently observed (10-15).

Flavonoids like rutin may prevent endothelial dysfunction via scavenging radicals, chelating metal ions and having anti-inflammatory and anti-thrombotic activities $(16,17)$. Next to this, they could also prevent damage by enhancing the release of vasodilatory factors including nitric oxide (NO), endothelium-derived hyperpolarizing factor and inhibit the release of proangiogenic factors including vascular endothelial growth factor and matrix metalloproteinase-2 (16). These direct effects depend on the concentration of rutin, which has a relatively short half-life in plasma of approximately 10 hours (18). However, to protect against CVD, rutin has to protect over a longer time span. Therefore, there has to be an additional effect. During, radical scavenging flavonoids are readily oxidized and are converted into quinones (19). It has been shown that these flavonoid quinones react selectively with thiol groups from cellular proteins (20), potentially also cellular thiolcontaining proteins involved in redox regulation. This might induce a long term protection. Such a long term protective effect of rutin, even when rutin is not present anymore, could explain why rutin intake can offer protection against CVD.

One of these potential targets of the flavonoid quinones is Kelch like ECH associated protein 1 (Keap1), Keap1 is a dimeric protein that functions as an inhibitor of nuclear factor (erythroid-derived 2)-like 2 (Nrf2). Keap1 consists of three domains, the $\mathrm{N}$ terminal BTB (Broad complex, Tramtrack, and Bric-a-Brac) domain, the intervening region and the C-terminal Kelch domain (21). The BTB domain is involved in the homodimerization of Keap1 and is known for stress sensing (22). Oxidative stress e.g. 
induced by $\mathrm{H}_{2} \mathrm{O}_{2}$ impairs the inhibitory activity of Keap1. In case of oxidative stress, Keap1's inhibitory activity of Nrf2 is lost, and newly synthesized Nrf2 can translocate to the nucleus, where it induces expression of endogenous antioxidant enzymes including the enzymes that are part of the glutathione and thioredoxin systems (23-27). Next to its role in the endogenous antioxidant system, recent findings also show that chemical modification of the selenocysteine of thioredoxin reductase 1 (TrxR1) apart from inhibiting its thioredoxin disulfide reductase activity, is accompanied by an upregulation of the expression of Nrf2-mediated genes (28).

Apart from Nrf2, also other transcription factors are redox regulated. One of these redox regulated transcription factors is nuclear factor kappa-light-chain-enhancer of activated $\mathrm{B}$ cells (NF-KB). NF-KB is involved in inflammation and consists of several subunits including p65 and p50 (29). Normally it is bound to and inhibited by Inhibitor of kappa-light-chain-enhancer of activated B cells (IKB). Oxidation of Cysteine 62 of its p50 subunit can prevent the NF-kB mediated expression of genes involved in inflammation for example Interleukin-6 (IL-6) and Interleukin-8 (IL-8) (29).

Another target for flavonoid quinones could be hypoxia inducible factor (HIF). HIF is a redox regulated transcription factor that is normally degraded via the proteasome. However, during hypoxia, it becomes hydroxylated and can translocate to the nucleus, where it induces genes involved in angiogenesis for example vascular endothelial growth factor (VEGF) (30).

Activation of Nrf2 by a flavonoid quinone will result in a relatively long lasting protection compared to their direct effects that depend on the scavenging by rutin. It might explain why the flavonoid has a protective effect even when it is not present anymore in the human body. The phenomenon that the induction of these diverse adaptive systems can protect against a future oxidative challenge, can be seen as hormesis or adaptation (31). Because in this case, a defined beneficial nutritional compound, the flavonoid rutin, is able to induce an adaptive defense mechanism, the endogenous antioxidant system, this type of hormesis, is called parahormesis $(32,33)$.

Could the dietary antioxidant rutin besides its direct protection against oxidative stress also protect vasorelaxation and induce an adaptation in endogenous antioxidant systems? Our hypothesis is that rutin acts in a dual manner, first it can directly protect by scavenging radicals and secondly the quinone formed in this process can selectively induce adaptation that provides protection over a longer period and thereby efficiently preventing impaired arteriolar relaxation. If so, the elegance of this mode of action also 
suggests that rutin becomes activated for its protective functions selectively in regions of increased oxidative stress.

\section{Material and methods}

\subsection{Compounds}

In all exposures, rutin (Sigma-Aldrich, St. Louis, MO, USA) is dissolved in 1:4 in 36 $\mathrm{mM} \mathrm{NaOH}$ and $145 \mathrm{mM}$ Potassium Phosphate Buffer $\mathrm{pH}=7.5$, unless stated otherwise. Ham's F12K medium, fetal bovine serum (FBS), penicillin, streptomycin, Hank's Balanced Salt Solution (HBSS), Minimal Essential Medium (MEM) and phosphate buffered saline (PBS) were derived from Gibco (Bleiswijk, The Netherlands). Recombinant selenocysteinecontaining rat (Rattus norvegicus) TrxR1 was obtained and isolated like previously described (34). FBS used for the HEK293 cells was obtained from PAA (Pasching, Austra); GE Healthcare and penicillin and streptomycin from Biochrom (Cambridge, united Kingdom). Endothelial cell growth supplement (ECGS) was ordered at BD Biosciences (Franklin Lakes, USA), heparin at LEO Pharma (Ballerup, Denmark), T75 collagen coated culture flasks at Greiner Bio-one (Alphen a/d Rijn, The Netherlands), Endothelial Basal Medium (EBM) at Lonza (Basel, Switzerland) and Eagle's Minimum Essential Medium (EMEM) from ATCC 30-2003 (Molsheim Cedex, France). TurboFect was obtained from Thermo Scientific (Bleiswijk, the Netherlands) and Invitrogen (Bleiswijk, The Netherlands) provided hygromycine B. Tumor necrosis factor $\alpha$ (TNF $\alpha$ ), tert-butylhydroquinone (tBHQ) and dimethyloxalylglycine, $\mathrm{N}$-(methoxyoxoacetyl)-glycine methyl ester (DMOG), DCFHDiacetate, black 96 well plate with clear bottom, hydrogen peroxide $\left(\mathrm{H}_{2} \mathrm{O}_{2}\right)$, horse radish peroxidase (HRP), tyrosinase, Tris- $\mathrm{HCl}$, ethylenedinitrilotetraacetic acid (EDTA), $\beta$ nicotinamide adenine dinucleotide $2^{\prime}$-phosphate reduced (NADPH), bovine serum albumin (BSA), reduced glutathione (GSH), auranofin, 5,5'-dithiobis(2-nitrobenzoic acid) (DTNB), dimethylsulfoxide (DMSO), ammoniumbicarbonate, trifluoroacetic acid (TFA), paraformaldehyde (PFA), 4-2-hydroxyethyl-1-piperazineethanesulfonic acid (HEPES), NaCl, $\mathrm{KCl}, \mathrm{MgSO}_{4} \cdot 7 \mathrm{H}_{2} \mathrm{O}, \mathrm{KH}_{2} \mathrm{PO}_{4}, \mathrm{CaCl}_{2}$, glucose, $\mathrm{NaHCO}_{3}, \mathrm{U} 46619$ and bradykinin were obtained from Sigma-Aldrich (St. Louis, MO, USA). Keap1 was ordered at Sino Biological (Beijing, China) and trypsin and protease max at Promega (Madison, USA). Biocoat provided collagen I-coated 96-well plates (354649, BD). DAPI was obtained from Thermo Scientific (Bleiswijk, the Netherlands). Qiagen supplied the Qiagen RNeasy Plus Mini Kit (Venlo, The Netherlands) and Biorad the $\mathrm{iQ}^{\mathrm{TM}} \mathrm{SYBR}^{\circledast}$ Green Supermix (Veenendaal, The Netherlands). 


\subsection{Rutin quinone formation}

Fifty $\mu \mathrm{M}$ rutin was incubated with $6.4 \mathrm{nM} \mathrm{HRP}$ and $50 \mu \mathrm{M} \mathrm{H} \mathrm{H}_{2}$ or $50 \mu \mathrm{M}$ rutin with $1000 \mathrm{U}$ tyrosinase. Then an UV absorption spectrum from $\lambda=200-500 \mathrm{~nm}$ was made by measuring for 30 minutes every minute using the spectrophotometer. Also the

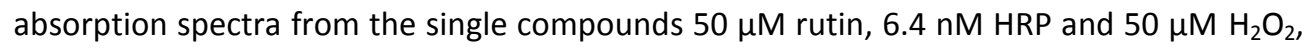
$50 \mu \mathrm{M}$ rutin and $50 \mu \mathrm{M} \mathrm{H}_{2} \mathrm{O}_{2}$, and $1000 \mathrm{U}$ tyrosinase were made.

\subsection{Cell Culture}

Human Umbilical Vein Endothelial Cells (HUVECs; ATCC CRL-1730) were cultured in Ham's F12K medium supplemented with $10 \%$ (v/v) FBS, $50 \mathrm{U} / \mathrm{ml}$ penicillin combined with $50 \mu \mathrm{g} / \mathrm{ml}$ streptomycin, $0.05 \mathrm{mg} / \mathrm{ml}$ endothelial cell growth supplement, and 0.01 $\mathrm{mg} / \mathrm{ml}$ heparin. T75 collagen coated culture flasks were used to culture the HUVECs. Passages of 16-24 were used to perform experiments. The endothelial phenotype of the cells was confirmed previously and did not differ between immortalized endothelial cells and primary endothelial cells $(35,36)$. All exposures were done in EBM.

Stable Human Embryonic Kidney cells (HEK293; ATCC CRL 3216) reporter cell line HEK(pTRAF ${ }^{\mathrm{Nrf2} / \mathrm{HIF} / \mathrm{NF}-\mathrm{KB}}$ ) was cultured in Eagle's Minimum Essential Medium supplemented with $10 \% \mathrm{FBS}$ and 100 units penicillin/ml and $100 \mu \mathrm{g}$ streptomycin/ml. Experiments were performed with cells from passage 14-15. Cells were incubated in an environment containing a humidified atmosphere containing $5 \% \mathrm{CO}_{2}$ and at $37^{\circ} \mathrm{C}$.

\subsection{Generation of stable HEK(pTRAF $\left.{ }^{\mathrm{Nr} 2 / \mathrm{HIF} / \mathrm{NF}-\mathrm{kB}}\right)$ reporter cell line}

HEK293 cells were seeded in 6-well plates at a density of 500,000 cells per well. One day after seeding the cells were transfected with plasmid for Transcription factor Reporter Activation based upon Fluorescence $\left(\mathrm{pTRAF}^{\mathrm{Nrf2/HIF/NF-kB}}{ }^{\text {) }}\right.$ (37). For these experiments a new updated $\mathrm{pTRAF}^{\mathrm{Nr} f 2 / \mathrm{HIF} / \mathrm{NF}-\mathrm{KB}}$ was used, in which we have exchanged the Cyan Fluorescent Protein (CFP) to the brighter Turquoise Fluorescent Protein (TFP) (38). For each well a mixture of $100 \mu \mathrm{l}$ OptiMem, $1 \mu \mathrm{l} \mathrm{pTRAF}{ }^{\mathrm{Nrf2} / \mathrm{HIF} / \mathrm{NF}-\mathrm{KB}}$-DNA and $2 \mu \mathrm{l}$ TurboFect was incubated at room temperature. After 20 minutes the DNA mixture was diluted with $1.15 \mathrm{ml}$ complete EMEM and added to the cells. To select for cells stably transfected with pTRAF $^{\text {Nrf2/HIF/NF-kB }}$ (37), $50 \mu \mathrm{g} / \mathrm{ml}$ Hygromycine B was added to the cell media, $72 \mathrm{~h}$ after transfection. After approximately 3 weeks, single clones were selected, expanded and tested for proper transcriptional response by exposing the cells to $30 \mu \mathrm{M}$ tBHQ (for Nrf2 response), $20 \mu \mathrm{g} / \mathrm{ml}$ TNF $\alpha$ (for NF-KB response) and $375 \mu \mathrm{M}$ DMOG (for HIF response) and activities were determined by fluorescence as described (37). Positive clones were 
expanded and frozen. Further detailed evaluation of this new HEK293(pTRAF ${ }^{\mathrm{Nr} 2 / \mathrm{HIF} / \mathrm{NF}-\mathrm{KB}}$ ) cell line is to be published elsewhere.

\subsection{Intracellular oxidative stress determination}

Intracellular oxidative stress levels were determined using the dichlorofluorescin (DCFH) probe that results in a fluorescent signal after oxidation by a wide range of Reactive Oxygen Species (ROS) (39). Despite several limitations (40) like its limited specificity to measure $\mathrm{H}_{2} \mathrm{O}_{2}$, the DCFH assay is still appropriate to measure general oxidative stress $(41,42)$. Since rutin scavenges different kinds of ROS including $\mathrm{H}_{2} \mathrm{O}_{2}$, superoxide and peroxynitrite (43-46), the unspecific nature of the DCFH assay is actually an advantage, because the fluorescence is in this way also a good way to determine the scavenging capacity of rutin. The DCFH assay has been used successfully before for other flavonoids (47). A stock solution of $10 \mathrm{mM}$ DCFH-Diacetate was made in DMSO, dilutions were made in EBM medium. For direct measurements, HUVECs were plated at a density of 40,000 cells in a black 96 well plate with clear bottom. Next, cells were incubated for 45 minutes with $20 \mu \mathrm{M}$ DCFH-Diacetate at $37{ }^{\circ} \mathrm{C}$ and $5 \% \mathrm{CO}_{2}$. Then, cells were washed using HBSS. Cells were incubated with 0-30 $\mu \mathrm{M}$ rutin or a combination of 0-30 $\mu \mathrm{M}$ rutin and 200 $\mu \mathrm{M} \mathrm{H}_{2} \mathrm{O}_{2}$. Every 5 minutes the fluorescence was determined for a period of 20 minutes on a microplate reader (Spectramax microplate reader, Molecular devices, Sunnyvale, USA) using an excitation wavelength of $\lambda=485 \mathrm{~nm}$ and measuring emission at $\lambda=538 \mathrm{~nm}$. To determine adaptation, cells were first incubated with 0-30 $\mu \mathrm{M}$ rutin or a combination of 0 $30 \mu \mathrm{M}$ rutin and $200 \mu \mathrm{M} \mathrm{H} \mathrm{O}_{2}$ for 3 hours (Figure 1).

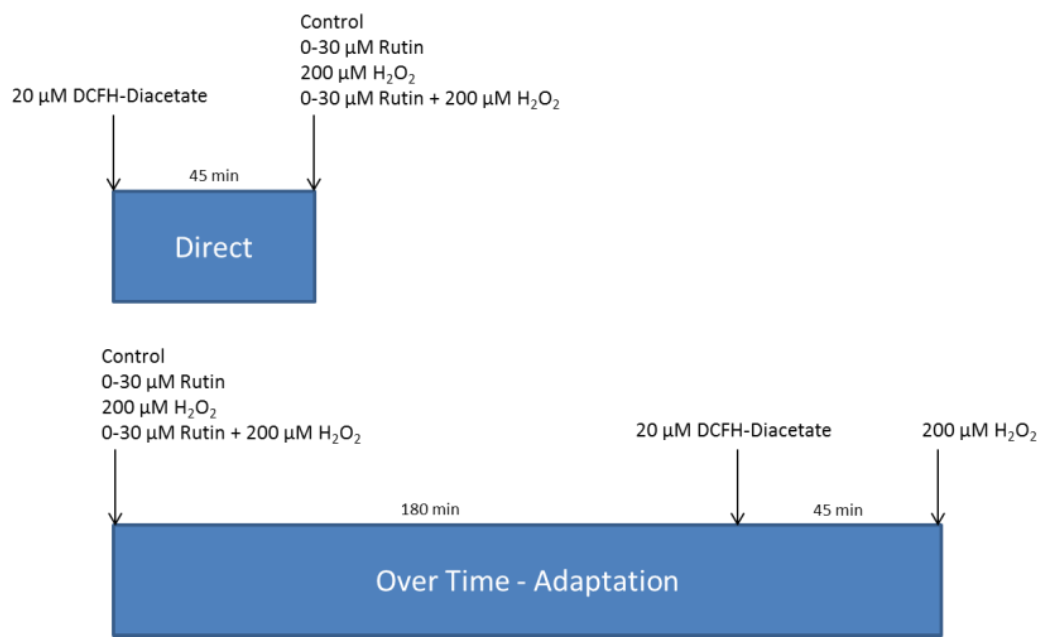

Figure 1: Exposure scheme to determine the direct and over time (adaptation) in HUVECs using the DCFH assay to measure oxidative stress. 
Then, cells were rinsed with HBSS and $20 \mu \mathrm{M}$ DCFH-Diacetate was added. After 45 minutes at $37{ }^{\circ} \mathrm{C}$ and $5 \% \mathrm{CO}_{2}$, a washing step was performed and cells were exposed to a second challenge of $200 \mu \mathrm{M} \mathrm{H}_{2} \mathrm{O}_{2}$. Finally, fluorescence of DCF was measured on the microplate reader (Ex $485 \mathrm{~nm}, \mathrm{Em} 538 \mathrm{~nm}$ ) and the level of oxidative stress was determined. Adaptation was calculated by relating the level of oxidative stress in HUVECS with pretreatment to the level of oxidative stress in HUVECs without pretreatment. The latter cells are only exposed to the second challenge of $200 \mu \mathrm{M} \mathrm{H}_{2} \mathrm{O}_{2}$.

\subsection{Thioredoxin reductase 1 activity}

The direct NADPH-dependent DTNB reduction assay (48) was used, with experiments performed in $50 \mathrm{mM}$ Tris- $\mathrm{HCl}$ pH7.5, $2 \mathrm{mM}$ EDTA buffer at room temperature. Pure recombinant rat TrxR1 (150 nM) was incubated with $250 \mu \mathrm{M} \mathrm{NADPH}, 0.1 \mathrm{mg} / \mathrm{ml}$ BSA and different concentrations of compounds for 15 minutes. During this incubation the absorbance was measured at $\lambda=340 \mathrm{~nm}$ using a Spectramax microplate reader (Molecular Devices) using the SoftMax Pro software. The experiments were at least done in triplicate in 96 well plates. These experiments were first without and then in presence of $1 \mathrm{mM} \mathrm{GSH}$. The different concentrations of the compounds were $25 \mathrm{U}$ tyrosinase, $150 \mathrm{nM}$ auranofin, $10 \mu \mathrm{M}$ rutin, $50 \mu \mathrm{M}$ rutin, $10 \mu \mathrm{M}$ rutin and $25 \mathrm{U}$ tyrosinase, $50 \mu \mathrm{M}$ rutin and $25 \mathrm{U}$ tyrosinase. Subsequently, the samples were diluted 20 times in $50 \mathrm{mM}$ Tris- $\mathrm{HCl}$ pH7.5, 2 mM EDTA buffer containing $50 \mathrm{mM}$ NAPDH. Then $2.5 \mathrm{mM}$ DTNB dissolved in ethanol was added and the absorbance was measured at $\lambda=412 \mathrm{~nm}$ for 15 minutes.

\subsection{Liquid Chromatography - Mass Spectrometry}

Fifty $\mu \mathrm{M} \mathrm{H} \mathrm{H}_{2} \mathrm{O}_{2}$ and $6.4 \mathrm{nM} \mathrm{HRP}$ were used to oxidize $50 \mu \mathrm{M}$ rutin (from stock solution of $2 \mathrm{mM}$ dissolved in ethanol) in $50 \mathrm{mM}$ ammoniumbicarbonate buffer $\mathrm{pH} 7.4$. Incubation was performed at 37 o $C$ for 15 minutes in the presence of $0.1 \mathrm{mg} / \mathrm{ml}$ Keap1. Additionally, Keap1 was incubated with either $50 \mu \mathrm{M} \mathrm{H}_{2} \mathrm{O}_{2}$ and $6.4 \mathrm{nM} \mathrm{HRP}$ or $50 \mu \mathrm{M}$ rutin or nothing (control). Subsequently, $1 \mu \mathrm{l} 1 \mu \mathrm{g} / \mu \mathrm{l}$ trypsin and $1 \mu \mathrm{l} 1 \mu \mathrm{g} / \mu \mathrm{l}$ protease max were added to $50 \mu \mathrm{l}$ sample and incubated at 37 ㅇ $\mathrm{C}$ for 120 minutes. Next, samples are diluted 1:10 in $98 \% \mathrm{H}_{2} \mathrm{O}, 2 \%$ acetonitrile and $0.05 \%$ TFA. The Q Exactive hybrid quadrupoleOrbitrap mass spectrometer with a nano-electrospray Flex ion source (Proxeon), connected to a UHPLC Dionex Ultimate 3000 by Thermo Scientific (Waltham, MA, USA) was used to perform LC-MS/MS measurements. For the UHPLC a C18-reversed phase column (Thermo Scientific Accliam Pepmap C18 column, 75- $\mu \mathrm{m}$ inner diameter $\times 15 \mathrm{~cm}$, $5 \mu \mathrm{m}$ particle size) was used. A 30 minutes linear gradient of $4-45 \%$ Buffer B (80\% acetonitrile and $0.08 \%$ formic acid) was applied to separate peptides at a flow rate of 300 $\mathrm{nL} / \mathrm{min}$. A data-dependent top10 method was used to obtain MS data. The most abundant 
precursor ions were dynamically chosen from the survey scan (250-1250 m/z) in positive mode. Survey scans were acquired at a resolution of 70,000 and the dynamic exclusion duration was $30 \mathrm{~s}$. A $4.0 \mathrm{~m} / \mathrm{z}$ window was applied to isolate precursors. Settings to obtain higher-energy collisional dissociation (HCD) were put at a resolution of 17,500 and, normalized collision energy of $30 \mathrm{eV}$. The under fill ratio was defined as $1.0 \%$. The peptide recognition mode was enabled to run the instrument, but singly charged and charge states of more than five were excluded. Identification was done by searching the data against the Uniprot database using the Sequest HT Proteome Discoverer 1.4 search engine (Thermo Scientific). The false discovery rate (FDR) was set to 0.01 for proteins and peptides, which had to have a minimum length of 6 amino acids. The precursor mass tolerance was set at $10 \mathrm{ppm}$ and the fragment tolerance at $0.2 \mathrm{Da}$. One miss-cleavage was tolerated, oxidation of methionine and rutin (608.138) modification on a cysteine were set as a dynamic modification.

\subsection{Assessment of Nrf2, HIF and NF-KB activity using the HEK(pTRAF $^{\mathrm{Nrf2} / \mathrm{HIF} / \mathrm{NF}-\mathrm{kB}}$ ) reporter cell line}

Stably transfected HEK(pTRAF $F^{\mathrm{Nr} 2 / \mathrm{HIF} / \mathrm{NF}-\mathrm{KB}}$ ) were seeded at a density of 18,000 cells/well in collagen I-coated 96 -well plates. Then rutin ( $2 \mathrm{mM})$ was prepared by dissolving it in ethanol. Cells were exposed to $20 \mathrm{ng} / \mathrm{ml}$ TNF $\alpha, 30 \mu \mathrm{M} \mathrm{tBHQ}$ and $375 \mu \mathrm{M}$ DMOG for 24 hours. Additionally, cells are exposed to $5 \mu \mathrm{M}$ rutin, $25 \mathrm{U}$ tyrosinase or a combination of $5 \mu \mathrm{M}$ rutin and $25 \mathrm{U}$ filtered tyrosinase for 45 minutes in MEM. Then, medium is exchanged to EMEM supplemented with $10 \%$ FBS and antibiotics and kept in the incubator for the remaining time. After 24 hours, exposed cells were fixed in $2 \%$ icecold PFA for 10 minutes at room temperature and were stained with $0.02 \mu \mathrm{g} / \mathrm{ml} \mathrm{DAPI}$ for 30 minutes. Finally, PBS was added to the wells and the plates were stored at 4 o $C$ until analyses. Subsequently analyses were done with the Operetta high-content imaging system (PerkinElmer) (37). Seven fields in each well were measured with the Operetta using the channels for mCherry, Ypet, TFP/CFP, DAPI and bright field, with the excitation and emission filters and exposure times set as reported earlier (37). To analyze the data, first, Sternberg's rolling ball method was used to subtract the background by the ImageJ software. Columbus software (PerkinElmer) was used for further analysis. DAPI stained nuclei were used to identify individual cells and the merged channel for all fluorescent proteins was used to detect corresponding cytosols. In the defined cell area the intensity of mCherry, Ypet, TFP/CFP were quantified as an indirect measurement of transcriptional activity of Nrf2, HIF and NF-KB respectively. Constitutively expressing vectors of single fluorescent proteins (mCherry, Ypet, CFP) were used to estimate the background cutoff fluorescent levels as described (37). Only cells showing intensity above the background were used for further analysis. After correction for this background, the sum of the 
measured intensity of mCherry, Ypet and TFP/CFP was divided by the amount of cells determined.

\subsection{Gene expression of Glutamate Cysteine Ligase, catalytic subunit}

HUVECs were plated at a density of 200,000 cells/well in 6-well plates and incubated for 24 hours. Then, cells were preincubated with or without $3 \mu \mathrm{M}$ rutin. Next, $100 \mu \mathrm{M} \mathrm{H}_{2} \mathrm{O}_{2}$ was added to half of the wells of each group, resulting in four experimental conditions: control, $3 \mu \mathrm{M}$ rutin, $100 \mu \mathrm{M} \mathrm{H}_{2} \mathrm{O}_{2}$ and $3 \mu \mathrm{M}$ rutin $+100 \mu \mathrm{M} \mathrm{H}_{2} \mathrm{O}_{2}$. After 3 hours of incubation RNA was isolated using the Qiagen RNeasy Plus Mini Kit. The quantity of RNA was determined using the nanodrop (Thermo scientific nanodrop 1000 spectrophotometer, isogen life science, De Meern, The Netherlands). Complementary DNA (cDNA) was made by converting 500 ng RNA using iScript cDNA synthesis kit (Biorad, Veenendaal, The Netherlands). The $\mathrm{iQ}^{\mathrm{TM}} \mathrm{SYBR}^{\circledR}$ Green Supermix with the following primers: Glutamate Cysteine Ligase, catalytic subunit (GCLC; sense: 5'-GCACATCTACCACGCCGTC-3' and antisense: 5'-CCACCTCATCGCCCCAC-3') were used to perform a Quantitative RT-PCR in a Bio Rad MyiQ Real-Time PCR Detection System (American Laboratory Trading, Boston/Cambridge). As housekeeping gene $\beta$-actin was used ( $\beta$-actin; sense: $5^{\prime}$ CCTGGCACCCAGCACAAT-3' and antisense: 5'-GCCGATCCACACGGAGTACT-3'). Finally, to determine relative GCLC gene expression the $2^{-\triangle \triangle C T}$ method was applied (49).

\subsection{Tissue preparation of fetal placental vessels of the chorionic plate (50)}

Placentas were retrieved from women that delivered via spontaneous vaginal delivery at the Department of Gynecology and Obstetrics of Maastricht University Medical Center+ after a full-term pregnancy of 38-42 weeks. Patients were aged between 21-36 years, did not smoke and were not reported to be diagnosed with hypertension. During delivery no general anesthesia was applied to the patients. Patients gave permission to use their placenta's for research purposes (METC number: 13-4-102). Placentas $(n=6)$ were obtained at latest 60 minutes after delivery. Secondary branches of the human fetal placental arteries of the chorionic plate were isolated in HEPES (containing $143.3 \mathrm{mM} \mathrm{NaCl}$, $4.7 \mathrm{mM} \mathrm{KCl}, 1.2 \mathrm{mM} \mathrm{MgSO}_{4} \cdot 7 \mathrm{H}_{2} \mathrm{O}, 1.2 \mathrm{mM} \mathrm{KH}_{2} \mathrm{PO}_{4}, 2.5 \mathrm{mM} \mathrm{CaCl}_{2}, 5.5 \mathrm{mM}$ glucose, $15 \mathrm{mM}$ HEPES) at room temperature and stored at 4 ㅇ $\mathrm{C}$ in HEPES for maximum 12 hours. 


\subsection{Recording vasomotor responses}

After 12 hours $2 \mathrm{~mm}$ arteriole segments were mounted on a wire-myograph (DMT, Aarhus, Denmark) and experiments were performed in $7 \mathrm{ml}$ Krebs Ringer Bicarbonate solution (KRB, containing $118.5 \mathrm{mM} \mathrm{NaCl}, 4.7 \mathrm{mM} \mathrm{KCl}, 1.2 \mathrm{mM} \mathrm{MgSO} \cdot 7 \mathrm{H}_{2} \mathrm{O}$, $1.2 \mathrm{mM} \mathrm{KH}_{2} \mathrm{PO}_{4}, 25 \mathrm{mM} \mathrm{NaHCO}, 2.5 \mathrm{mM} \mathrm{CaCl}_{2}, 5.5 \mathrm{mM}$ glucose) at 37 o $\mathrm{C}$ and aerated with $95 \% \mathrm{O}_{2} / 5 \% \mathrm{CO}_{2}$. L-arginine was not added to the buffer, since addition of L-arginine did not enhance eNOS activity and subsequent vasorelaxation, since L-arginine is present in plasma and tissue (endothelium) in concentrations at least 50 times higher than the $\mathrm{Km}$ of the NOS enzyme (50-54).

The segments were actively stretched to a diameter at which a maximal contractile response was obtained with $40 \mathrm{mM} \mathrm{KCl}$ (containing $43.2 \mathrm{mM} \mathrm{KCl}, 1.2 \mathrm{mM}$ $\mathrm{MgSO}_{4} \cdot 7 \mathrm{H}_{2} \mathrm{O}, 1.2 \mathrm{mM} \mathrm{KH}_{2} \mathrm{PO}_{4}, 25 \mathrm{mM} \mathrm{NaHCO}, 2.5 \mathrm{mM} \mathrm{CaCl}, 5.5 \mathrm{mM}$ glucose, $80.6 \mathrm{mM}$ $\mathrm{NaCl})(55,56)$.

Then, two conditions were applied. For both conditions, a contraction was induced by adding 3 nM U46619 that incubated for approximately 20 minutes until stabile contraction was achieved. Subsequently, a Concentration Response Curve (CRC) was generated by adding $1 \mathrm{nM}-0.1 \mathrm{mM}$ bradykinin. Next, a washing step was performed using KRB buffer. Complete relaxation was achieved about 20-30 min after the washing step.

Then, only for the second condition $10 \mu \mathrm{M}$ of rutin was added. Thereafter, vessels were exposed to $100 \mu \mathrm{M} \mathrm{H}_{2} \mathrm{O}_{2}$ for 30 minutes. After washing, rutin was again added for condition 2 followed by a contraction with U46619 and the CRC with bradykinin in both conditions (Figure 2).

For condition 1 a similar protocol was applied, with the exception of the addition of rutin that was left out.

Contractile responses to $\mathrm{U} 46619$ (3 $\mathrm{nM}$ ) and relaxing responses to increasing concentrations of bradykinin ( $1 \mathrm{nM}-0.1 \mathrm{mM})$ were recorded in the pre and post exposure to $\mathrm{H}_{2} \mathrm{O}_{2}(100 \mu \mathrm{M})$ (Figure 2). The effects of rutin $(10 \mu \mathrm{M})$ on contractile responses to $\mathrm{U} 46619$ ( $3 \mathrm{nM}$ ) and relaxing responses to bradykinin (1 nM-0.1mM), in the pre and post exposure to $\mathrm{H}_{2} \mathrm{O}_{2}(100 \mu \mathrm{M})$ were evaluated (Figure 2). Relaxations to bradykinin were expressed relative to contraction of $U 46619$ (3 nM), as described earlier $(57,58)$. 


\subsection{Statistics}

All experiments were at least performed in duplicates at $\mathrm{n} \geq 3$ and all data are represented as mean \pm SEM. To test differences between the control an pretreated secondary placental human arteries of the chorionic plate a Two-Way Anova was used. Additionally, independent samples with equal variances were assessed for statistical significance with a t-test. P-values $<0.05$ were considered to be statistically significant.
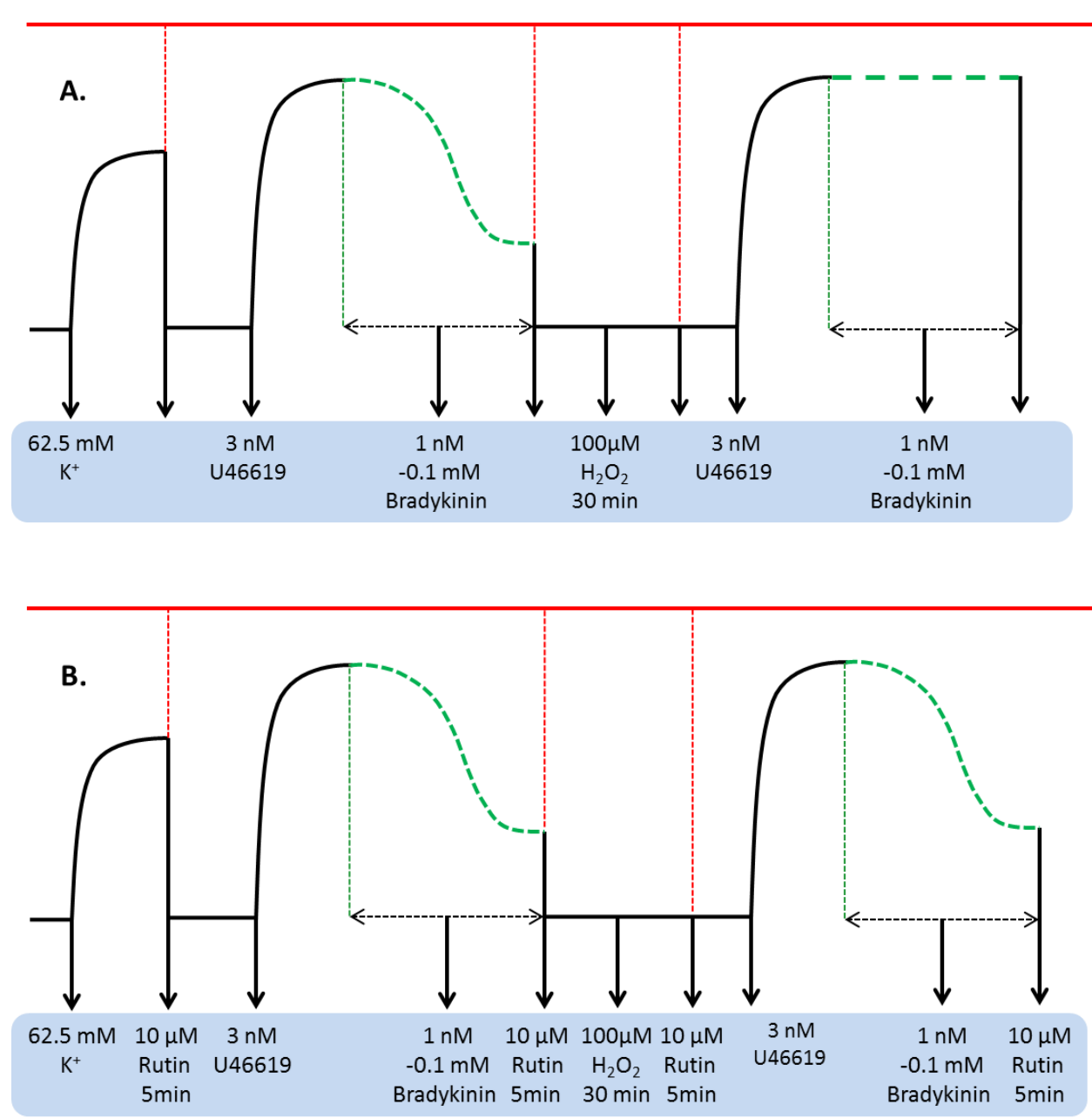

Figure 2: Typical tracings of the two different conditions applied to human fetal placental arteries of the chorionic plate. 


\section{Results}

\subsection{Rutin is oxidized to rutin quinone}

By monitoring the UV absorption spectra of rutin preparations in solution it was found that rutin $(50 \mu \mathrm{M})$ does not spontaneously oxidize at neutral $\mathrm{pH}$, to any apparent degree. In cells, rutin quinone can however be generated using treatment with either $\mathrm{H}_{2} \mathrm{O}_{2}$ or tyrosinase $(59,60)$. In a test tube, we found that under the conditions used here, rutin did not react directly with $\mathrm{H}_{2} \mathrm{O}_{2}$ (Figure $3 \mathrm{~A}$ ).
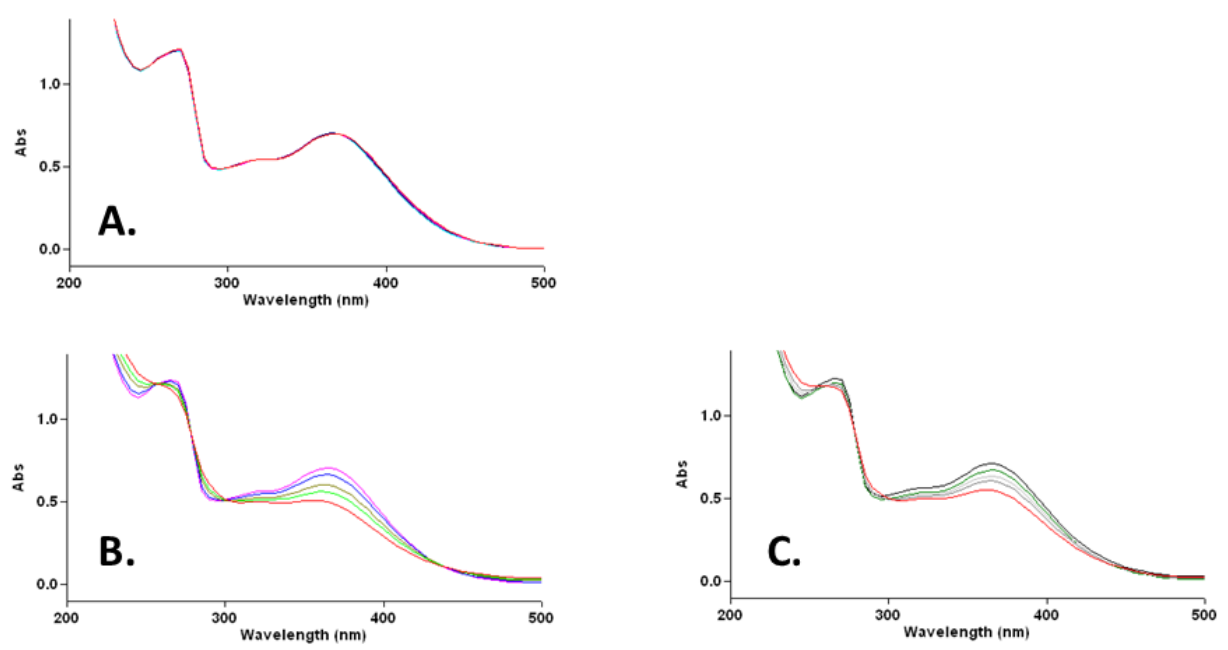

D.
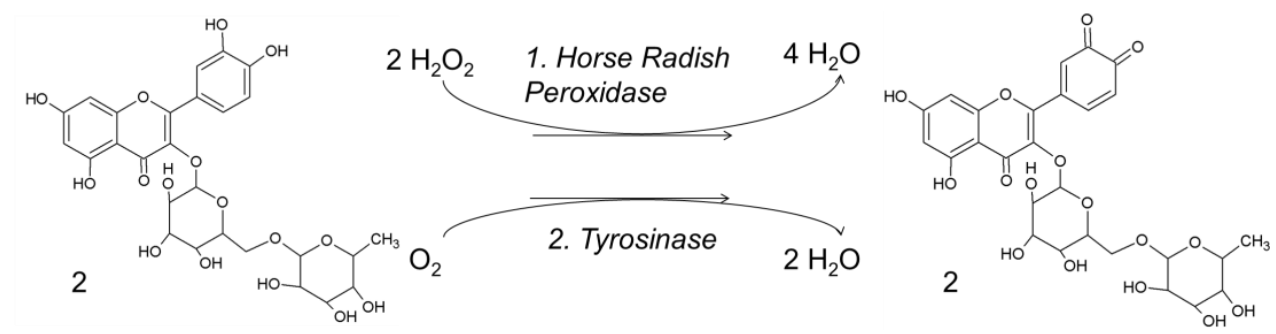

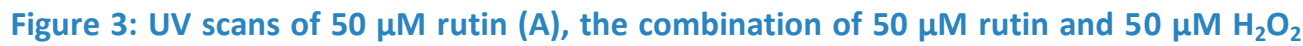

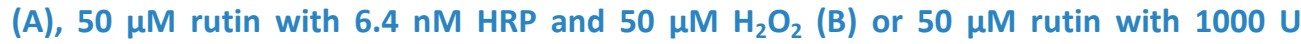
tyrosinase (C). HRP and $\mathrm{H}_{2} \mathrm{O}_{2}$ alone or tyrosinase alone did not give an absorption spectrum. The UV scans were measured every minute for 5 minutes after starting the reaction with addition of rutin (purple scans were the first and red the last). Structural display of the formation of rutin quinone (D) from rutin by $\mathrm{HRP}$ and $\mathrm{H}_{2} \mathrm{O}_{2}$ or tyrosinase. 
A solution of $\mathrm{HRP}$ and $\mathrm{H}_{2} \mathrm{O}_{2}$ or tyrosinase without rutin did not give spectra at the wavelengths analyzed here, but the combination of $\mathrm{HRP}(6.4 \mathrm{nM})$ and $\mathrm{H}_{2} \mathrm{O}_{2}(50 \mu \mathrm{M})$ or tyrosinase $(1000 \mathrm{U})$ with rutin changed within 5 minutes at a similar rate the UV spectrum of rutin into the spectrum of rutin quinone (Figure $3 \mathrm{~B}$ and $\mathrm{C}$ ). This indicated that either $\mathrm{HRP}$ and $\mathrm{H}_{2} \mathrm{O}_{2}$ or tyrosinase treatment can be used to generate rutin quinone (Figure 3D).

\subsection{Rutin directly scavenges ROS as indicated by DCF fluorescence}

To initiate analyses of the redox effects of rutin in a cellular system, the direct antioxidant activity of rutin was first measured using the DCFH assay with HUVECs. Concentrations of 0-30 $\mu \mathrm{M}$ rutin were used, because previously it has been shown that at these concentrations the flavonoid was not toxic towards these cells (42). Rutin without DCFH-DA did not affect DCF fluorescence compared to control (data not shown). Also in all experiments the vehicle (buffer (1:4 in $36 \mathrm{mM} \mathrm{NaOH}$ and $145 \mathrm{mM}$ Potassium Phosphate Buffer $\mathrm{pH}=7.5$ or other dissolvent) diluted in the exposure medium) had no effect on the examined parameter (data not shown). Addition of $200 \mu \mathrm{M} \mathrm{H}_{2} \mathrm{O}_{2}$ significantly increased the cellular levels of ROS, as indicated by DCF fluorescence, while rutin was found to concentration dependently decrease the DCF signal, indicative of lower ROS levels (Figure 4). Also in cells that were not exposed to additional $\mathrm{H}_{2} \mathrm{O}_{2}$, rutin induced a concentration dependent decrease in the DCF signal compared to control cells (Figure 4).

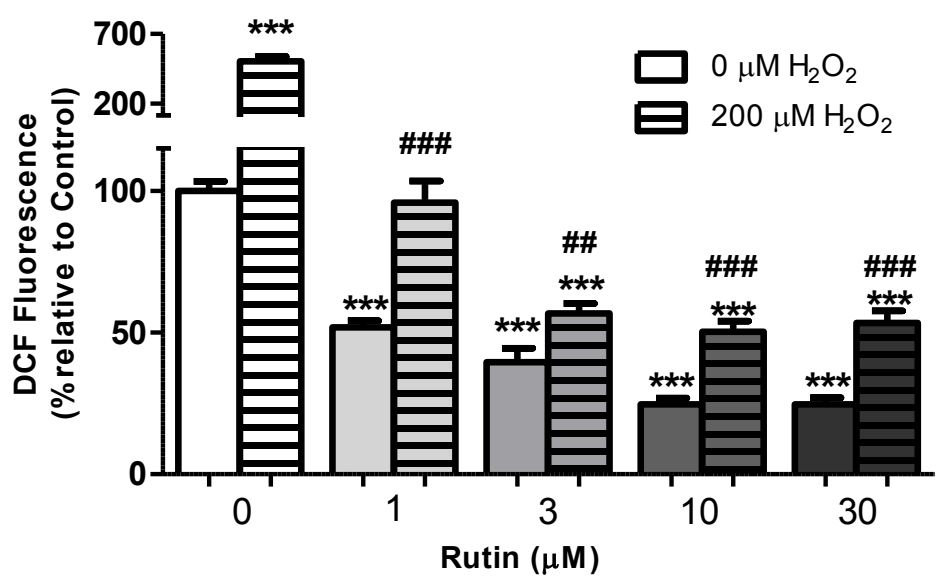

Figure 4: ROS levels as indicated by DCF fluorescence in HUVECs exposed to 0-30 $\mu \mathrm{M}$ rutin and/or $200 \mu \mathrm{M} \mathrm{H}_{2} \mathrm{O}_{2}$ for 20 minutes. $\mathrm{N}=3$ and data are presented as mean \pm SEM. *P $<0.05$; ** $<$ < 0.01; ***P < 0.001 compared to control, \#\#P<0.01, \#\#\#< 0.001 compared to the same condition but without exposure to $200 \mu \mathrm{M} \mathrm{H}_{2} \mathrm{O}_{2}$. 


\subsection{Rutin pretreatment induces adaptation of HUVECs to oxidative stress}

Next to the direct antioxidant effect of rutin, the potency of rutin to provide protection by lowering ROS levels over time was investigated. HUVECs were pretreated for 3 hours with $200 \mu \mathrm{M}$ of $\mathrm{H}_{2} \mathrm{O}_{2}$ in combination with $3 \mu \mathrm{M}$ rutin, giving rutin quinone. After this pretreatment, the cells were exposed to $200 \mu \mathrm{M} \mathrm{H}_{2} \mathrm{O}_{2}$ for 20 minutes. The DCF signal in the pretreated cells was significantly lower than in control cells, indicating that the levels of ROS induced were less than without pretreatment, suggestive of an adaptive response induced by rutin quinone (Figure 5). Pre-incubating HUVECs with only $3 \mu \mathrm{M}$ rutin or only $200 \mu \mathrm{M} \mathrm{H}_{2} \mathrm{O}_{2}$ also decreased the DCF signal as triggered by a second challenge with $\mathrm{H}_{2} \mathrm{O}_{2} 3$ hours later, but not as potently as the combination of rutin and $\mathrm{H}_{2} \mathrm{O}_{2}$.

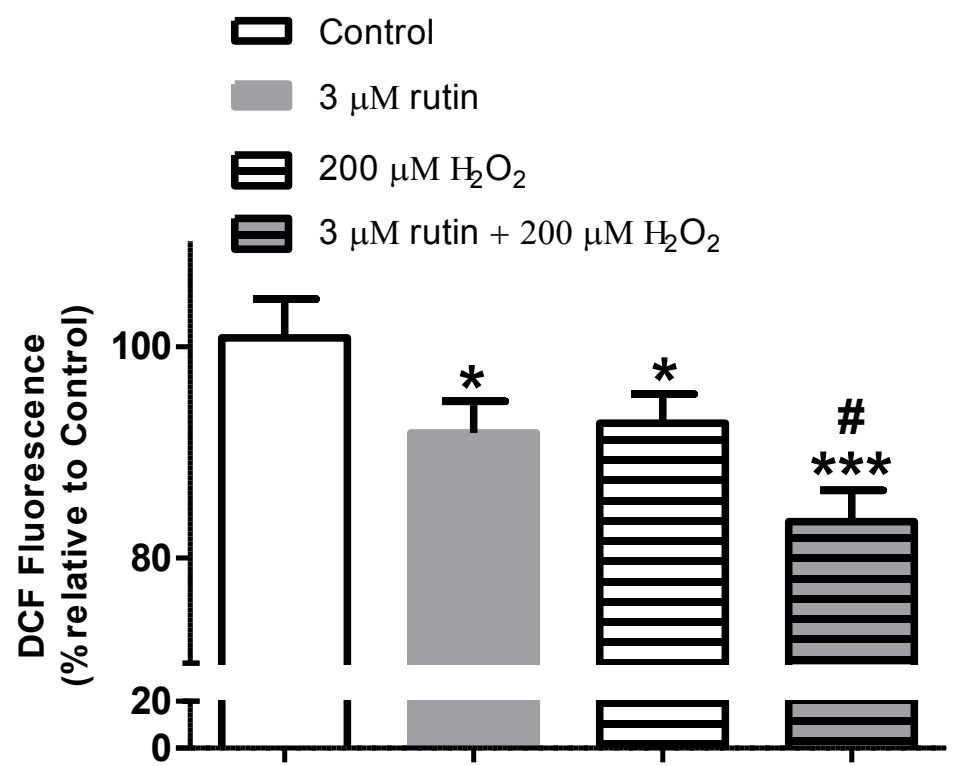

Figure 5: ROS levels as indicated by DCF fluorescence in HUVECs pretreated with either 0

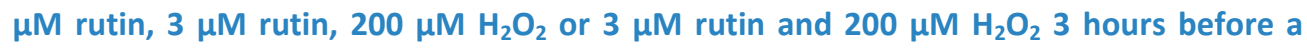
second treatment with $200 \mu \mathrm{M} \mathrm{H} \mathrm{H}_{2}$ for 20 minutes. It was found that ROS levels induced by $\mathrm{H}_{2} \mathrm{O}_{2}$ were less in cells pretreated with $\mathrm{H}_{2} \mathrm{O}_{2}$, rutin, or the combination relative to control (no rutin or $\mathrm{H}_{2} \mathrm{O}_{2}$ ). $\mathrm{N}=3$ and data are presented as mean \pm SEM. $* \mathrm{P}<$ 0.05 ; **P $<0.01 ; * * * \mathrm{P}<0.001$ compared to control, \#P<0.05 compared to the same condition but without exposure to $3 \mu \mathrm{M}$ rutin. 


\subsection{Rutin quinone can inhibit thioredoxin reductase and form an adduct with Keap1}

Because rutin quinone is an electrophile that can target nucleophiles in cells and since the selenoprotein thioredoxin reductase is a potential target that may be linked to Nrf2 activation (28) we first analyzed inhibition of this enzyme. This showed that rutin quinone can indeed inhibit thioredoxin reductase in a time- and concentration-dependent manner, but the enzyme can at least in vitro be protected from inhibition by GSH (Figure $6)$.
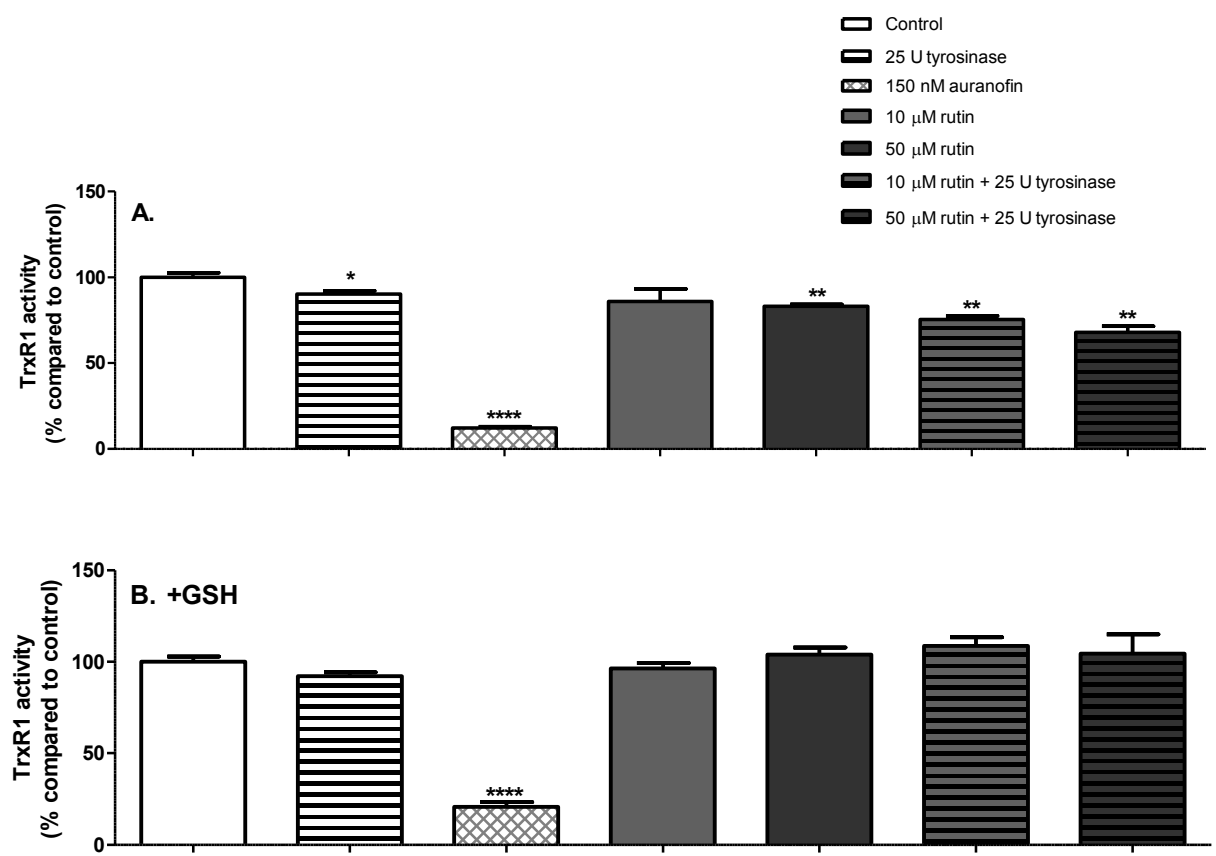

Figure 6: TrxR1 activity in percentage compared to control activity, after incubation with $25 \mathrm{U}$ tyrosinase, $150 \mathrm{nM}$ auranofin, $10 \mu \mathrm{M}$ rutin, $50 \mu \mathrm{M}$ rutin, $10 \mu \mathrm{M}$ rutin and $25 \mathrm{U}$ tyrosinase, $50 \mu \mathrm{M}$ rutin and $25 \mathrm{U}$ tyrosinase for 15 minutes. $\mathrm{N}=\mathbf{3}$ and data are presented as mean \pm SEM. $* \mathrm{P}<0.05 ; * * \mathrm{P}<0.01 ; * * * \mathrm{P}<0.001$ compared to control.

Another cellular protein that may be targeted by electrophiles and lead to Nrf2 activation is Keap1, which was analyzed using pure compounds and mass spectrometry. After incubating Keap1 with $50 \mu \mathrm{M}$ rutin in presence of $6.4 \mathrm{nM} \mathrm{HRP}$ and $50 \mu \mathrm{M} \mathrm{H}_{2} \mathrm{O}_{2}$ both $\mathrm{b}^{+}(\mathrm{red}), \mathrm{y}^{+}, \mathrm{y}^{2+}$ and $\mathrm{y}^{4+}$ (blue) ions were found of the expected MS/MS spectrum of the 
peptide fragment LIEFAYTASISMGEKCVLHVMAGHVMYQIDSVVR containing an adduct on cysteine 151 of rutin on Keap1 (Figure 7 and Supplementary Table 1). In the control, 50 $\mu \mathrm{M}$ rutin and $50 \mu \mathrm{M} \mathrm{H}_{2} \mathrm{O}_{2}$ with $6.4 \mathrm{nM}$ HRP conditions these fragments were not found (Supplementary Table 1). This indicates that rutin in the presence of $50 \mu \mathrm{M} \mathrm{H}_{2} \mathrm{O}_{2}$ and 6.4 nM HRP was converted into rutin quinone, which can react with cysteine 151 of Keap1 resulting in a rutin-Keap1 adduct. Thus, both targeting of TrxR1 and Keap1 by rutin quinone may occur. Next we analyzed whether Nrf2 activation may be detected in cells upon treatment with rutin with or without oxidative challenges that lead to rutin quinone formation.

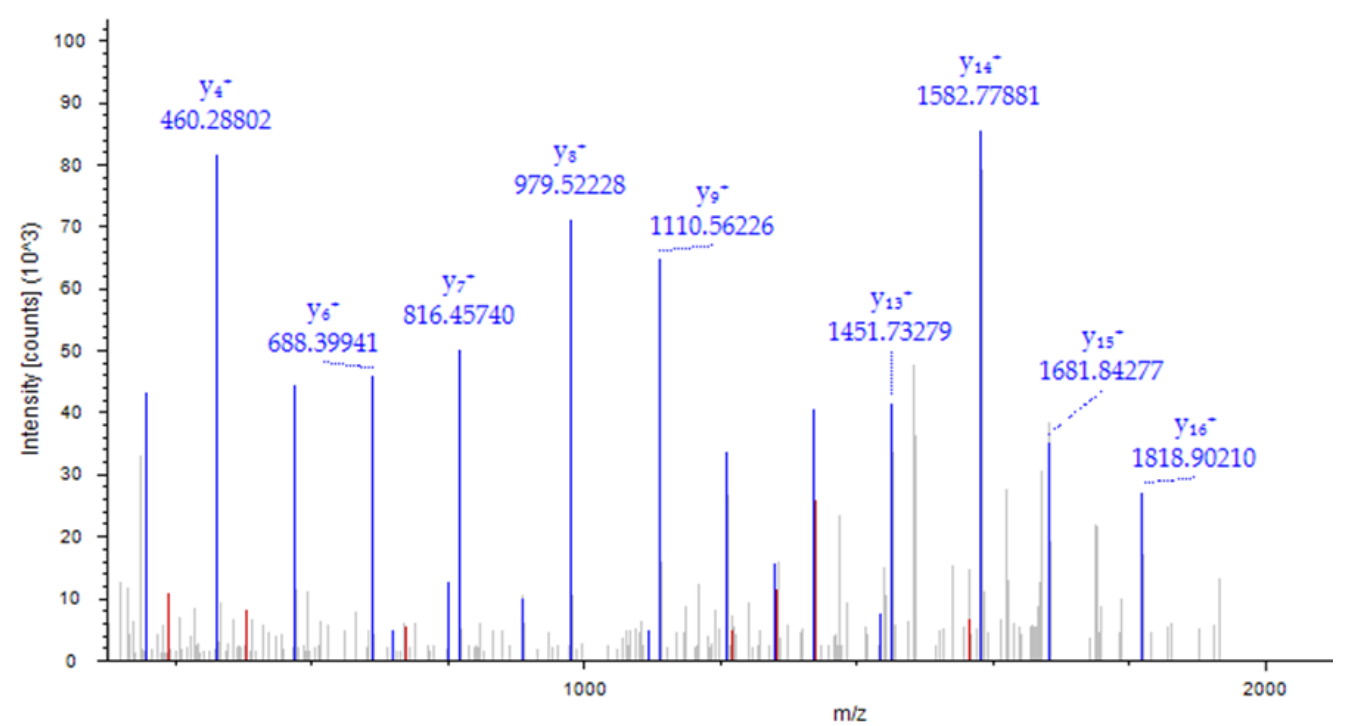

Figure 7: MS spectrum of Keap1 incubated with $50 \mu \mathrm{M}$ rutin, $50 \mu \mathrm{M} \mathrm{H} \mathrm{H}_{2} \mathrm{O}_{2}$ and $6.4 \mathrm{nM}$ HRP for 15 minutes. Typical example is shown.

\subsection{Rutin quinone activates Nrf2 but blunts HIF and NF-KB activation under conditions of oxidative stress in HEK(pTRAF $\left.{ }^{\mathrm{Nrf} 2 / \mathrm{HIF} / \mathrm{NF}-\mathrm{kB}}\right)$ reporter cells}

Not only nuclear presence, but also the ability of redox regulated trancription factors to bind their promotor sequence was analyzed by HEK(pTRAF ${ }^{\mathrm{Nr} 2 / \mathrm{HF} / \mathrm{NF}-\mathrm{kB}}$ ) reporter cells, stably expressing a vector containing response elements for Nrf2, HIF and NF-KB coupled to three different fluorescent proteins (See Methods section for details and references). Additionally also the effect of rutin on the simultaneous activation of and regulation of intermittent crosstalk (29) between redox regulated transcription factors could be determined. Incubation for 24 hours with typical inducers of Nrf2, HIF and NF-KB 
e.g $30 \mu \mathrm{M} \mathrm{tBHQ}, 375 \mu \mathrm{M}$ DMOG or $20 \mathrm{ng} / \mathrm{ml} \mathrm{TNF} \alpha$, respectively, confirmed the activation of these transcription factors (Figure 8). Next to DMOG, $30 \mu \mathrm{M}$ tBHQ and $20 \mathrm{ng} / \mathrm{ml}$ TNF $\alpha$ were also able to induce activation of HIF in normoxia, which is in agreement with previous findings $(37,61)$.
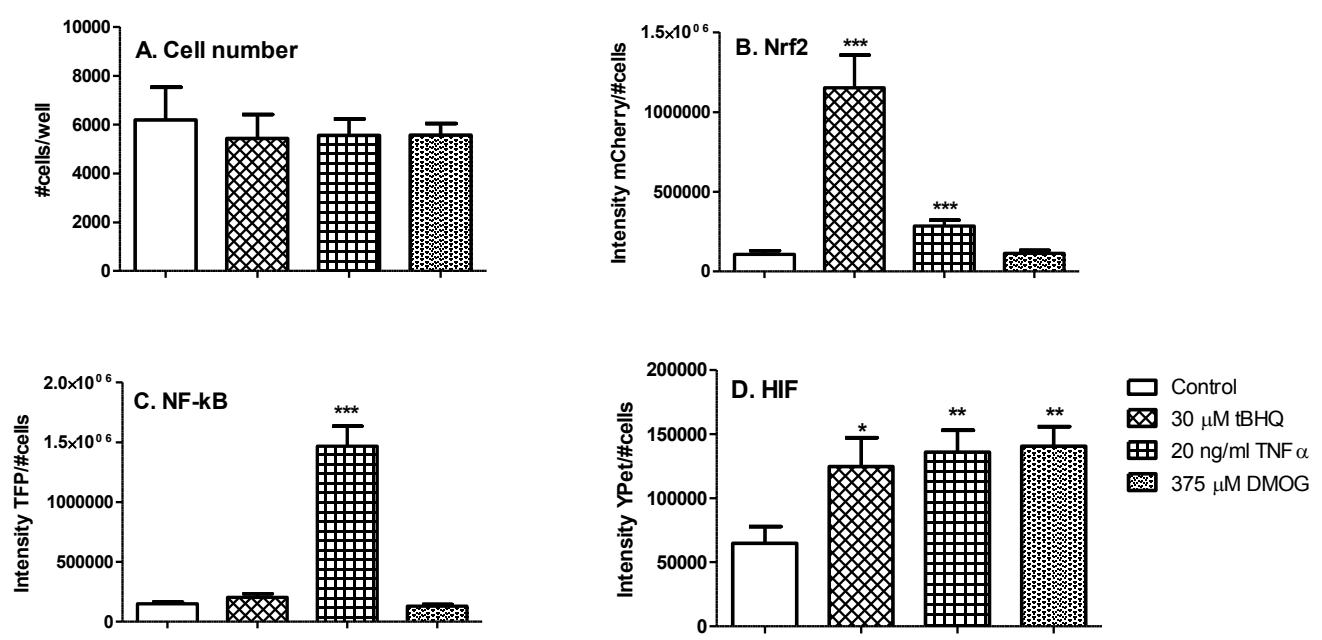

Figure 8: Nrf2, HIF and NF-KB activity using of HEK(pTRAF ${ }^{\mathrm{Nrf2} / \mathrm{HIF} / \mathrm{NF}-\mathrm{KB}}$ ) reporter cell line exposed to indicated drugs for $\mathbf{2 4}$ hours. A) No effect of the indicated incubations on cell number, measured by DAPI is seen. B) $30 \mu \mathrm{M}$ tBHQ increases Nrf2 activity, shown by an increase in mCherry fluorescent protein intensity compared to control. C) $20 \mathrm{ng} / \mathrm{ml} \mathrm{TNF \alpha}$ increases NF-KB activity, shown by an increase in TFP fluorescent protein intensity compared to control. D) $375 \mu \mathrm{M}$ DMOG, $30 \mu \mathrm{M}$ tBHQ and $20 \mathrm{ng} / \mathrm{ml}$ TNF $\alpha$ increase HIF activity, shown by an increase in YPet fluorescent protein intensity compared to control. $\mathrm{N}=3$ and data are presented as mean \pm SEM. ${ }^{*} \mathrm{P}<0.05 ; * * \mathrm{P}<0.01 ; * * \mathrm{P}<0.001$ compared to control.

Upon treatment with rutin in the absence of excessive oxidative stress, no significant effect was seen for any of the transcription factors (Figure 9). However, using treatment with $25 \mathrm{U}$ tyrosinase to increase cellular oxidative stress, this resulted in a significant decrease in cell number (Figure 9A). At the same time a significant increase in mCherry intensity, reflective of Nrf2 activity, was seen (Figure 9B), which was accompanied with significantly increased NF-KB and HIF activities (Figure $9 \mathrm{C}$ and D). Interestingly, by also including $5 \mu \mathrm{M}$ rutin under such conditions, although cell numbers were not improved (Figure 9A), the increase in Nrf2 activation was further exaggerated (Figure 9B) while the increased activities of NF-KB and HIF at the same time were blunted by rutin quinone compared to the treatment with tyrosinase alone (Figure $9 \mathrm{C}$ and $\mathrm{D}$ ). 

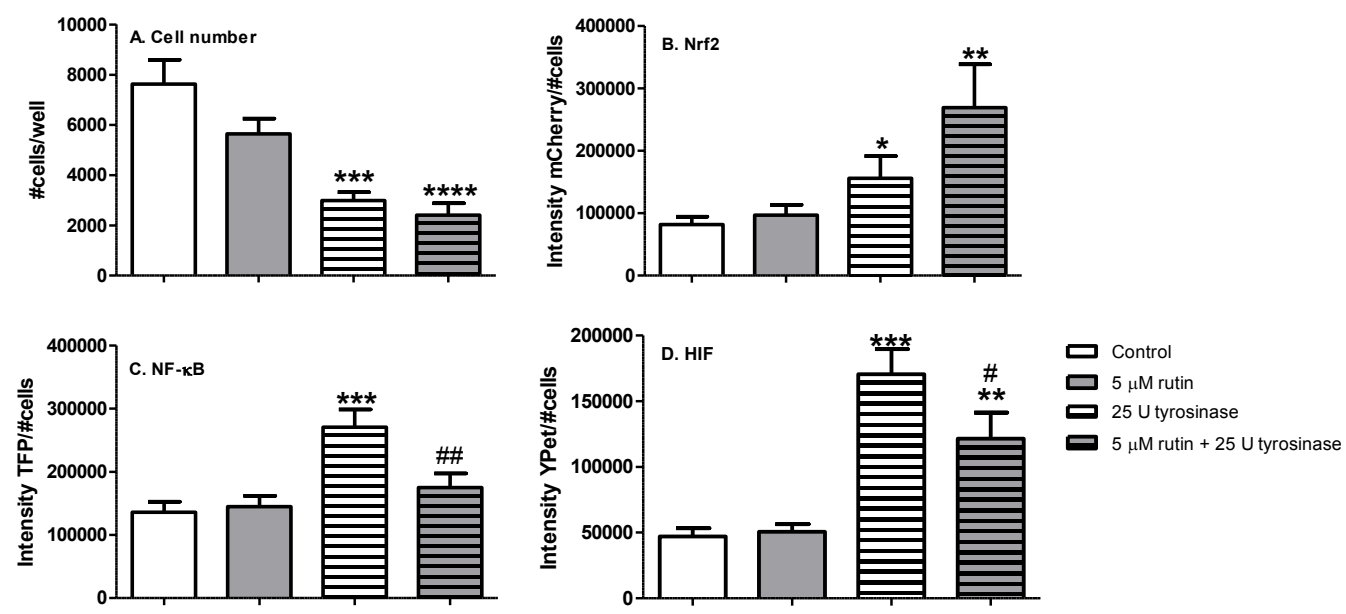

Figure 9: Nrf2, HIF and NF-KB activity in a stable HEK(pTRAF ${ }^{\mathrm{Nrf2} / \mathrm{HIF} / \mathrm{NF}-\mathrm{KB}}$ ) reporter cell line exposed to indicated drugs for 24 hours. A) Tyrosinase results in a decrease in cell number determined by DAPI. B) Both tyrosinase and rutin quinone increase Nrf2 activity. C) Tyrosinase increases NF-KB activity, whereas no effects of rutin in combination with tyrosinase are seen. D) Tyrosinase and rutin in presence of tyrosinase both show an increase in YPet intensity indicating that HIF activity is upregulated. $\mathrm{N}=3$ and data are presented as mean \pm SEM. ${ }^{*} \mathrm{P}<0.05 ; * * \mathrm{P}<0.01 ; * * * \mathrm{P}<0.001$ compared to control, \#P<0.05; \#\#P 0.01 compared to the same condition but without exposure to $3 \mu \mathrm{M}$ rutin.

\subsection{Rutin quinone induces expression of GCLC, a Nrf2-downstream target gene}

To confirm whether the increase in Nrf2 activity found in the stable HEK(pTRAF ${ }^{\mathrm{Nrf2} / \mathrm{HIF} / \mathrm{NF}-\mathrm{kB}}$ ) reporter cells could also be detected in HUVECs, the expression of a typical Nrf2 regulated GCLC, was determined. Exposing to $3 \mu \mathrm{M}$ rutin and $100 \mu \mathrm{M} \mathrm{H}_{2} \mathrm{O}_{2}$ for 3 hours indeed resulted in an increase in GCLC expression that was higher than that seen only using $\mathrm{H}_{2} \mathrm{O}_{2}$ (Figure 10). 


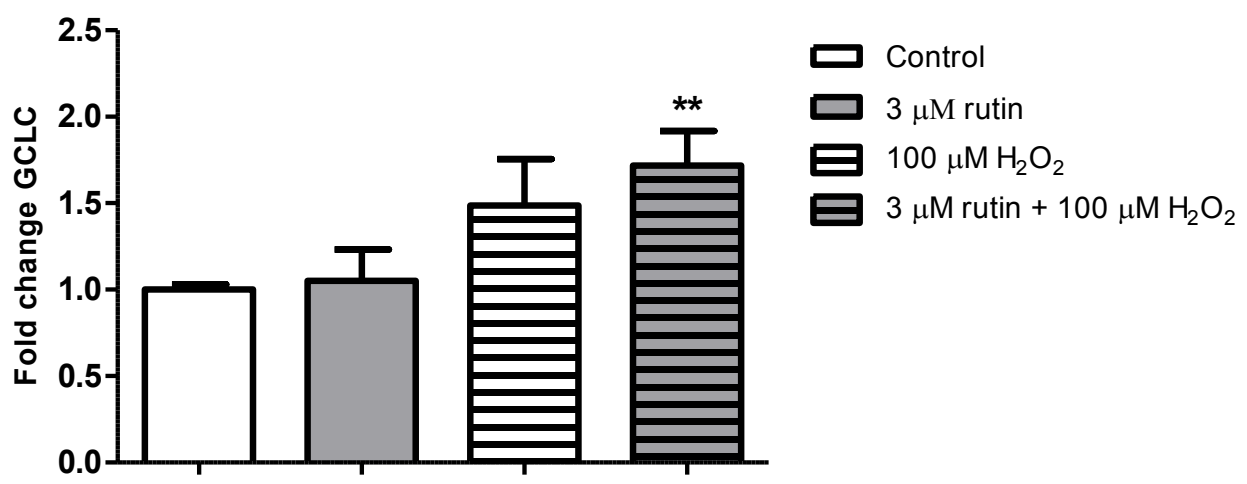

Figure 10: Glutamate Cysteine Ligase, catalytic subunit (GCLC) mRNA increases in HUVECs exposed to $3 \mu \mathrm{M}$ rutin and $100 \mu \mathrm{M} \mathrm{H} \mathrm{H}_{2} \mathrm{O}_{2}$ for 3 hours. $\mathrm{N}=3$ and data are presented as mean \pm SEM. $* * P<0.01$ compared to control.

\subsection{Rutin protects against impaired arteriolar vasorelaxation induced by $\mathrm{H}_{2} \mathrm{O}_{2}$ in fetal placental vessels of the chorionic plate}

In order to assess the effects of rutin on vasomotor responses in a human model for development of endothelial dysfunction and hypertension without interference of the autonomic nerve system (62-64), fetal placental arteries of the chorionic plate were isolated and mounted on the wire-myograph. The average optimal diameter of the arteriolar segments is $676 \mu \mathrm{m} \pm 1.9 \times 10^{2} \mu \mathrm{m}$ and the diameter did not differ significantly between the different samples. The treatments used to assess vasomotor responses are outlined in Figure 2.

First we treated the vessels with U46619, a vasoconstrictor analog of prostaglandin $\mathrm{PGH}_{2}$. The contractile response to $3 \mathrm{nM}$ U46619 was approximately $2.4 \pm 1.0$ $\mathrm{N} \mathrm{m}^{-1}$. The contraction induced by $\mathrm{U} 46619$ was not significantly affected by exposures to $\mathrm{H}_{2} \mathrm{O}_{2}$ with or without rutin.

Rutin alone did not have any significant effect on either U46619-mediated contraction or bradykinin-mediated relaxation. Addition of $100 \mu \mathrm{M} \mathrm{H} \mathrm{O}_{2}$ for 30 minutes, significantly obviated the relaxation induced by bradykinin (Figure 11A). Remarkably, however, treatment with $10 \mu \mathrm{M}$ rutin and $100 \mu \mathrm{M} \mathrm{H} \mathrm{O}_{2}$ for 30 minutes prevented the decrease in relaxation due to $\mathrm{H}_{2} \mathrm{O}_{2}$ and ensured that the relaxation induced by bradykinin remained (Figure 11B). This indicates that rutin protects against impaired arteriolar vasorelaxation as otherwise triggered by $\mathrm{H}_{2} \mathrm{O}_{2}$. 

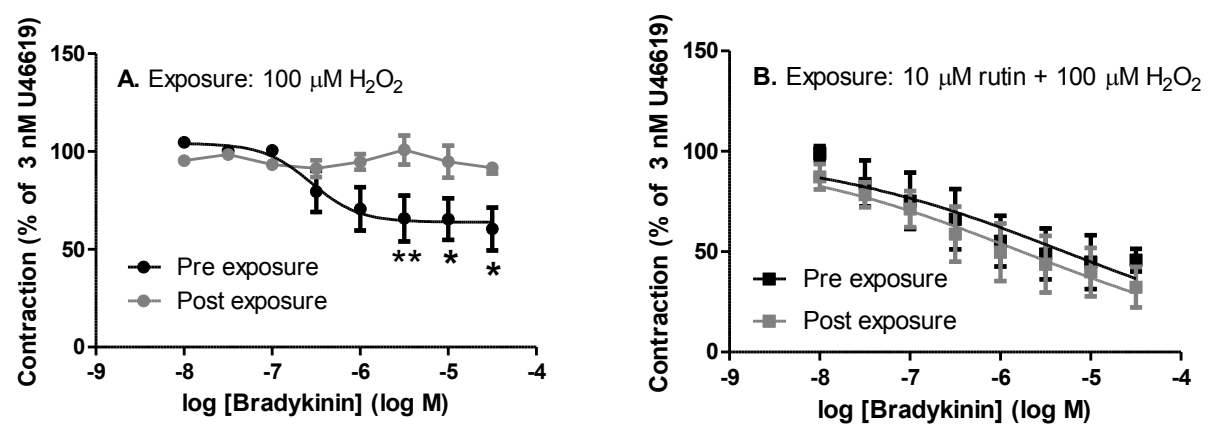

Figure 11: Bradykinin relaxation shown as percentage of the contraction induced by 3 nM U46619 in different human fetal placental secondary arteries of the chorionic plate before (pre exposure) and after (post exposure) exposure to $100 \mu \mathrm{M} \mathrm{H}_{2} \mathrm{O}_{2}$ (A) or $100 \mu \mathrm{M}$ $\mathrm{H}_{2} \mathrm{O}_{2}$ with $10 \mu \mathrm{M}$ rutin (B) for 30 minutes. $\mathrm{N}=3$ and data are presented as mean $\pm S E M$. *P $<0.05 ; * * \mathrm{P}<0.01 ; * * * \mathrm{P}<0.001$ compared to control.

\section{Discussion}

Rutin protects against the development of CVD, in which we examined the role of the antioxidant effect of rutin. The antioxidant effect of rutin is confirmed in the present study by the protection of HUVECs against oxidative stress afforded by $1 \mu \mathrm{M}$ rutin, a concentration that can be achieved in blood via the diet $(2,4,42,65-68)$. With supplementation even higher concentrations can be achieved (2). Also the quinone of flavonoids bound to GSH could be found in the bile of healthy human volunteers supplemented with flavonoid (69). In order to generate the quinone, rutin can scavenge different kind of ROS including $\mathrm{H}_{2} \mathrm{O}_{2}$, superoxide and peroxynitrite, which is in this study in cells induced by either $\mathrm{H}_{2} \mathrm{O}_{2}$ or tyrosinase (43-46). In humans, flavonoids are metabolized mainly by methylation (15\%) or glucuronidation (26\%) (70). From the metabolized flavonoids it has been found that they can also be converted into the quinone form and react with thiol containing proteins (20). HPLC analysis in Caco-2 cells after exposure to rutin showed that indeed the flavonoids are taken up by the cell and it is stated that unknown transporters should be involved (71-74). However, availability of rutin and the direct antioxidant effect do not explain protection on a longer term, since the half-life of rutin is too short to be able to provide direct protection by scavenging radicals. There has to be an additional mechanism to explain the long-lasting protective effect of rutin.

Intriguingly, our study shows that in presence of oxidative stress rutin appears to upregulate endogenous antioxidant systems, a form of adaptation or hormesis. In order to 
investigate this adaptation, HUVECs were pretreated with rutin, oxidative stress $\left(\mathrm{H}_{2} \mathrm{O}_{2}\right)$ and the combination of rutin and $\mathrm{H}_{2} \mathrm{O}_{2}$. Three hours later cells were exposed to oxidative stress $\left(\mathrm{H}_{2} \mathrm{O}_{2}\right)$ for 20 minutes and the level of ROS is determined. Pretreatment with rutin or $\mathrm{H}_{2} \mathrm{O}_{2}$ separately decreases the oxidative stress experienced 3 hours later due to a second treatment with $\mathrm{H}_{2} \mathrm{O}_{2}$ compared to control cells (no pretreatment). However, the decrease in intracellular oxidative stress in cells pretreated with the combination of rutin and $\mathrm{H}_{2} \mathrm{O}_{2}$ was significantly lower than in cells pretreated with one of the two compounds (rutin or $\mathrm{H}_{2} \mathrm{O}_{2}$ ) separately. This indicates that rutin and $\mathrm{H}_{2} \mathrm{O}_{2}$ is more effective in inducing adaptation than $\mathrm{H}_{2} \mathrm{O}_{2}$ or rutin alone. It has been reported that $\mathrm{H}_{2} \mathrm{O}_{2}$ can induce adaptation (75). The adaptation induced by rutin alone could be explained by oxidation of rutin by the basal level of oxidative stress.

Apparently, cells pretreated with rutin and $\mathrm{H}_{2} \mathrm{O}_{2}$ are more protected against a future challenge with oxidative stress. A molecular explanation for this finding could be that in conditions of oxidative stress rutin is oxidized to a quinone that induces a protective mechanism. This also explains the protective effect of rutin seen in other studies $(66,76-81)$, rutin may have been activated upon oxidative stress, it is not rutin as such that induces protection, but its oxidized product, rutin quinone, although this has not been previously recognized. More specifically rutin quinone might target Keap1 or TrxR1. For flavonoid quinones, it has been shown that these quinones can react selectively with thiol groups from essential cellular proteins (20). One of these thiol-containing proteins present in the cell could be Keap1, that is part of an important redox regulating system, the Keap1-Nrf2 pathway (82). Our study shows that rutin quinone can target a cysteine that is important in stress sensing, indicating that adduct formation of rutin on Keap1 will deteriorates Keap1's inhibition of Nrf2. Additionally, TrxR1, which is also linked to Nrf2 activation (28), thioredoxin disulfide reductase activity has been shown to be inhibited by rutin quinone. These findings are confirmed by the results that rutin quinone is also able to significantly increase Nrf2 activity. In this case tyrosinase was used to trigger oxidative stress and convert rutin into rutin quinone. Both $\mathrm{H}_{2} \mathrm{O}_{2}$ and HRP or tyrosinase induce a comparable formation of rutin quinone (Figure 3). Tyrosinase is an oxidase that has also been used previously to oxidize flavonoids (59). Importantly, rutin quinone induces an increase in expression of Nrf2-mediated genes including GCLC, whereas rutin alone did not show such effects. Furthermore, $\mathrm{H}_{2} \mathrm{O}_{2}$ also increases GCLC expression, but stronger GCLC expression is seen after incubation with rutin quinone. This confirms that rutin apart from its direct antioxidant effect, also can induce Nrf2-mediated expression of endogenous antioxidant genes. So the scavenging of rutin has two effects. First of all it gives a direct protection as confirmed by the DCFH experiments. Secondly, rutin quinone is formed that induces adaptation. Rutin quinone thus protects important cellular molecules against 
oxidative damage by upgrading the antioxidant activity of the cell, i.e. by selectively inducing an Nrf2-mediated increase in expression of endogenous antioxidant enzymes. Remarkably, the antioxidant expression induced by rutin quinone is higher than induced by $\mathrm{H}_{2} \mathrm{O}_{2}$. Both the direct protective and adaptive effect of rutin could be involved in the prevention of impaired vasorelaxation induced by $\mathrm{H}_{2} \mathrm{O}_{2}$ we observed in human arterioles. In addition, rutin in combination with $\mathrm{H}_{2} \mathrm{O}_{2}$ has been shown to induce an increase in NO production, eNOS gene expression, protein synthesis and activity in HUVECs on the long term (83), which is probably due to the formation of rutin quinone although this has not been previously recognized. These effects on NO and eNOS most likely further contribute to the vasorelaxating effect we found in human placental arterioles. Remarkably, the maximal vasorelaxation induced by bradykinin of $50 \%$, is comparable as seen in previous studies (84) and is accompanied by concomitant effects on the laminar flow and resistance of the vessels (85).

By exposing the Nrf2, HIF and NF-KB reporter cell line HEK(pTRAF $\left.F^{\mathrm{Nr} 2 / \mathrm{HF} / \mathrm{NF}-\mathrm{kB}}\right)$ to oxidative stress induced by tyrosinase a reduced cell number was shown, while rutin alone or the combination of $\mathrm{H}_{2} \mathrm{O}_{2}$ with rutin did not show effects on cell number. As response to the induced oxidative stress a significant activation of Nrf2 could be detected in cells exposed to tyrosinase. Additionally to activation of Nrf2 both HIF and NF-KB were activated after tyrosinase exposure, which could be explained by the induction of ROS by tyrosinase that activates HIF and NF-KB in normoxic conditions (86-88). Rutin alone had no effect on activation of Nrf2, HIF and NF-KB $(79,89,90)$. Notably, combining tyrosinase with rutin additionally induced Nrf2 but blunted the activity of HIF and NF-KB, indicating antiinflammatory effect of Nrf2 activity. Previously, from tyrosinase it was found that tyrosinase generates hydroxyl radicals, which can help explaining the increase in Nrf2, HIF and NF-KB activity found in this study (91), since ROS are known to induce both the HIF and NF-KB pathway $(92,93)$. An increase in HIF or NF-KB mediated inflammation is associated with a bad prognosis of patients with CVD (94). Our study shows that rutin prevents ROS-induced activation of the HIF and NF-KB pathway. This might also contribute to the protective effect of rutin and other antioxidants against CVD development because they can reduce the level of damaging ROS (95). Furthermore, flavonoids are reported to might have an anti-inflammatory activity (96-98), which corresponds with our findings that they do not enhance HIF and NF-KB activity.

The finding that the beneficial nutritional compound rutin has to be converted into a quinone by oxidative stress to be able to induce an increase in expression of endogenous antioxidant enzymes that protect against a future challenge with oxidative stress can be seen as a form of adaptation (31). This adaptation or hormesis, established by induction of Nrf2-mediated endogenous antioxidant system has also previously been 
seen for electrophiles like the aldehyde acrolein (99). However, with regard to rutin, this type of adaptation is called parahormesis instead of hormesis $(32,33)$.

Thus, rutin quinone is able to induce a protective, adaptive response e.g. by inducing the expression of Nrf2-mediated GCLC. GCLC is the rate-limiting enzyme of GSH synthesis, an important endogenous antioxidant system. An upregulation of this enzyme has previously been shown to be associated with an increase in GSH synthesis and accompanying GSH levels (100). Various in vivo human and animal studies show that thiol supplementation with $\mathrm{N}$-acetylcysteine, S-nitrosothiols or inducing an increase in GSH levels by enhancing its synthesis results in an enhanced function of nitroderivates like sodium nitroprusside and increases NO bioavailability, enhances vasodilatory effects and prevents endothelial dysfunction $(4,101-106)$. This implicates that the increase in GSH synthesis by the flavonoid rutin in the presence of oxidative stress and the increased GSH level that protects the endothelium and maintain its vasodilatory function could be linked. Future experiments should confirm that the increase in intracellular GSH causes the reduced risk on developing CVD in patients that took rutin supplements.

\section{Conclusion}

Rutin can protect against oxidative stress directly by its scavenging ability and indirectly via the formation of rutin quinone. Rutin quinone targets Keap1 and TrxR1 and results in adaptation by upregulating Nrf2-mediated endogenous antioxidant systems. The Nrf2-mediated increase in endogenous antioxidant enzymes like GCLC has previously been shown to lead to an increased level of GSH. This is targeted protection since it only happens in regions of high oxidative stress. It can explain why despite of its short half-life, rutin can have beneficial effects on a long term and reduces the risk on developing CVD. This is also confirmed by the finding that rutin protects against oxidative stress-induced impaired relaxation of human placental arterioles in which GSH can play an important role. Additionally, there are indications that rutin quinone could be anti-inflammatory. These mechanisms contribute to the further understanding why rutin can reduce the risk on developing CVD . 


\section{Supplement}

\subsection{Supplementary Results}

Supplementary Table 1: Keap1-rutin adduct formation in different incubation conditions. In black, the ions of the expected MS/MS spectrum of the peptide fragment LIEFAYTASISMGEKCVLHVMNGAVMYQIDSVVR are shown. In red the found $\mathrm{b}^{+}$, and blue the found $\mathrm{y}^{+}, \mathrm{y}^{2+}$ and $\mathrm{y}^{4+}$ ions are shown of this peptide fragment after incubating Keap1 with $50 \mu \mathrm{M}$ rutin, $50 \mu \mathrm{M} \mathrm{H}_{2} \mathrm{O}_{2}$ and $6.4 \mathrm{nM}$ HRP for 15 minutes. For the other conditions control Keap1, Keap1 with $50 \mu \mathrm{M}$ rutin or Keap1 with $50 \mu \mathrm{M} \mathrm{H}_{2} \mathrm{O}_{2}$ and $6.4 \mathrm{nM}$ HRP these ions could not be found.

\begin{tabular}{|c|c|c|c|c|c|c|c|c|}
\hline \multirow{2}{*}{$\begin{array}{l}\text { Conditions } \\
\text { Presence } \\
\text { of rutin- } \\
\text { Keap1 } \\
\text { adduct }\end{array}$} & \multicolumn{2}{|c|}{ Control Keap1 } & \multicolumn{2}{|c|}{ Keap1 + rutin } & \multicolumn{3}{|c|}{ Keap1 + HRP + $\mathrm{H}_{2} \mathrm{O}_{2}$} & $\begin{array}{l}\text { Keap1 + HRP } \\
\mathrm{H}_{2} \mathrm{O}_{2}+\text { rutin }\end{array}$ \\
\hline & & & & & & & $x$ & \\
\hline $\mathbf{b}^{+}$ & $b^{2+}$ & $b^{3+}$ & $b^{4+}$ & Seq. & $\mathrm{y}^{+}$ & $y^{2+}$ & $y^{3+}$ & $y^{4+}$ \\
\hline 114.09 & 57.549 & 38.701 & 29.278 & $\mathrm{~L}$ & & & & \\
\hline 135 & 31 & 97 & 29 & & & & & \\
\hline 227.17 & 114.09 & 76.396 & 57.549 & I & 4269.9 & 2135.4 & 1423.9 & 1068.2 \\
\hline 542 & 135 & 66 & 31 & & 2644 & 6686 & 8033 & 3707 \\
\hline 356.21 & 178.61 & 119.41 & 89.809 & $\mathrm{E}$ & 4156.8 & 2078.9 & 1386.2 & 1039.9 \\
\hline 802 & 265 & 086 & 96 & & 4237 & 2482 & 8564 & 6605 \\
\hline 503.28 & 252.14 & 168.43 & 126.57 & $\mathrm{~F}$ & 4027.7 & 2014.4 & 1343.2 & 1007.7 \\
\hline 644 & 686 & 366 & 707 & & 9977 & 0352 & 7144 & 0540 \\
\hline 574.32 & 287.66 & 192.11 & 144.33 & $A$ & 3880.7 & 1940.8 & 1294.2 & 970.93 \\
\hline 356 & 542 & 270 & & & 3135 & 6931 & 4863 & 830 \\
\hline 737.38 & 369.19 & 246.46 & 185.10 & $Y$ & 3809.6 & 1905.3 & 1270.5 & 953.17 \\
\hline 688 & 708 & 714 & 218 & & 9423 & 5075 & 6959 & 902 \\
\hline 838.43 & 419.72 & 280.14 & 210.36 & $T$ & 3646.6 & 1823.8 & 1216.2 & 912.41 \\
\hline
\end{tabular}


Rutin induces Nrf2-mediated adaptation in HUVECs exposed to oxidative stress

\begin{tabular}{|c|c|c|c|c|c|c|c|c|}
\hline 456 & 092 & 970 & 410 & & 3091 & 1909 & 1515 & 319 \\
\hline 909.47 & 455.23 & 303.82 & 228.12 & A & 3545.5 & 1773.2 & 1182.5 & 887.15 \\
\hline 168 & 948 & 874 & 338 & & 8323 & 9525 & 3259 & 127 \\
\hline 996.50 & 498.75 & 332.83 & 249.88 & S & 3474.5 & 1737.7 & 1158.8 & 869.39 \\
\hline 371 & 549 & 942 & 138 & & 4611 & 7669 & 5355 & 199 \\
\hline 1109.5 & 555.29 & 370.53 & 278.15 & I & 3387.5 & 1694.2 & 1129.8 & 847.63 \\
\hline 8778 & 753 & 411 & 240 & & 1408 & 6068 & 4288 & 398 \\
\hline 1196.6 & 598.81 & 399.54 & 299.91 & $S$ & 3274.4 & 1637.7 & 1092.1 & 819.36 \\
\hline 1981 & 354 & 479 & 041 & & 3001 & 1864 & 4819 & 296 \\
\hline 1327.6 & 664.33 & 443.22 & 332.67 & $M$ & 3187.3 & 1594.2 & 1063.1 & 797.60 \\
\hline 6031 & 379 & 495 & 053 & & 9798 & 0263 & 3751 & 495 \\
\hline 1384.6 & 692.84 & 462.23 & 346.92 & G & 3056.3 & 1528.6 & 1019.4 & 764.84 \\
\hline 8178 & 453 & 211 & 590 & & 5748 & 8238 & 5734 & 483 \\
\hline 1513.7 & 757.36 & 505.24 & 379.18 & $E$ & 2999.3 & 1500.1 & 1000.4 & 750.58 \\
\hline 2438 & 583 & 631 & 655 & & 3601 & 7164 & 5019 & 946 \\
\hline 1641.8 & 821.41 & 547.94 & 411.21 & K & 2870.2 & 1435.6 & 957.43 & 718.32 \\
\hline 1935 & 331 & 463 & 029 & & 9341 & 5034 & 599 & 881 \\
\hline 2352.9 & 1176.9 & 784.99 & 588.99 & C- & 2742.1 & 1371.6 & 914.73 & 686.30 \\
\hline 6628 & 8678 & 361 & 703 & Ruti & 9844 & 0286 & 766 & 507 \\
\hline 2452.0 & 1226.5 & 818.01 & 613.76 & V & 2031.0 & 1016.0 & 677.68 & 508.51 \\
\hline 3470 & 2099 & 642 & 413 & & 5151 & 2939 & 869 & 834 \\
\hline 2565.1 & 1283.0 & 855.71 & 642.03 & $\mathrm{~L}$ & 1931.9 & 966.49 & 644.66 & 483.75 \\
\hline 1877 & 6302 & 111 & 515 & & 8309 & 518 & 588 & 123 \\
\hline 2702.1 & 1351.5 & 901.39 & 676.29 & $\mathrm{H}$ & 1818.8 & 909.95 & 606.97 & 455.48 \\
\hline 7768 & 9248 & 741 & 988 & & 9902 & 315 & 119 & 021 \\
\hline 2801.2 & 1401.1 & 934.42 & 701.06 & V & 1681.8 & 841.42 & 561.28 & 421.21 \\
\hline 4610 & 2669 & 022 & 698 & & 4011 & 369 & 489 & 549 \\
\hline
\end{tabular}




\begin{tabular}{|c|c|c|c|c|c|c|c|c|}
\hline 2932.2 & 1466.6 & 978.10 & 733.82 & $M$ & 1582.7 & 791.88 & 528.26 & 396.44 \\
\hline 8660 & 4694 & 038 & 711 & & 7169 & 948 & 208 & 838 \\
\hline 3046.3 & 1523.6 & 1016.1 & 762.33 & $\mathrm{~N}$ & 1451.7 & 726.36 & 484.58 & 363.68 \\
\hline 2953 & 6840 & 1469 & 784 & & 3119 & 923 & 191 & 826 \\
\hline 3103.3 & 1552.1 & 1035.1 & 776.59 & G & 1337.6 & 669.34 & 446.56 & 335.17 \\
\hline 5100 & 7914 & 2185 & 321 & & 8826 & 777 & 760 & 752 \\
\hline 3174.3 & 1587.6 & 1058.8 & 794.35 & A & 1280.6 & 640.83 & 427.56 & 320.92 \\
\hline 8812 & 9770 & 0089 & 249 & & 6679 & 703 & 045 & 216 \\
\hline 3273.4 & 1637.2 & 1091.8 & 819.11 & V & 1209.6 & 605.31 & 403.88 & 303.16 \\
\hline 5654 & 3191 & 2370 & 959 & & 2967 & 847 & 141 & 288 \\
\hline 3404.4 & 1702.7 & 1135.5 & 851.87 & M & 1110.5 & 555.78 & 370.85 & 278.39 \\
\hline 9704 & 5216 & 0386 & 972 & & 6125 & 426 & 860 & 577 \\
\hline 3567.5 & 1784.2 & 1189.8 & 892.64 & $Y$ & 979.52 & 490.26 & 327.17 & 245.63 \\
\hline 6036 & 8382 & 5830 & 555 & & 075 & 401 & 843 & 565 \\
\hline 3695.6 & 1848.3 & 1232.5 & 924.66 & $\mathrm{Q}$ & 816.45 & 408.73 & 272.82 & 204.86 \\
\hline 1894 & 1311 & 4450 & 019 & & 743 & 235 & 399 & 982 \\
\hline 3808.7 & 1904.8 & 1270.2 & 952.93 & I & 688.39 & 344.70 & 230.13 & 172.85 \\
\hline 0301 & 5514 & 3919 & 121 & & 885 & 306 & 780 & 517 \\
\hline 3923.7 & 1962.3 & 1308.5 & 981.68 & D & 575.31 & 288.16 & 192.44 & 144.58 \\
\hline 2996 & 6862 & 8150 & 795 & & 478 & 103 & 311 & 415 \\
\hline 4010.7 & 2005.8 & 1337.5 & 1003.4 & $S$ & 460.28 & 230.64 & 154.10 & 115.82 \\
\hline 6199 & 8463 & 9218 & 4595 & & 783 & 755 & 079 & 742 \\
\hline 4109.8 & 2055.4 & 1370.6 & 1028.2 & V & 373.25 & 187.13 & 125.09 & 94.069 \\
\hline 3041 & 1884 & 1499 & 1306 & & 580 & 154 & 012 & 41 \\
\hline 4208.8 & 2104.9 & 1403.6 & 1052.9 & V & 274.18 & 137.59 & 92.067 & 69.302 \\
\hline \multirow[t]{3}{*}{9883} & 5305 & 3779 & 8016 & & 738 & 733 & 31 & 30 \\
\hline & & & & $\mathrm{R}$ & 175.11 & 88.063 & 59.044 & 44.535 \\
\hline & & & & & 896 & 12 & 50 & 20 \\
\hline
\end{tabular}




\section{References}

1. Sakac M, Pestoric M, Misan A, Nedeljkovic N, Jambrec D, Jovanov P, et al. Antioxidant Capacity, Mineral Content and Sensory Properties of Gluten-Free Rice and Buckwheat Cookies. Food technology and biotechnology. 2015 Mar;53(1):3847.

2. Carbonaro $\mathrm{M}$, Grant $\mathrm{G}$. Absorption of quercetin and rutin in rat small intestine. Annals of nutrition \& metabolism. 2005 May-Jun;49(3):178-82.

3. Manach C, Scalbert A, Morand C, Remesy C, Jimenez L. Polyphenols: food sources and bioavailability. The American journal of clinical nutrition. 2004 May;79(5):727-47.

4. Sharma S, Ali A, Ali J, Sahni JK, Baboota S. Rutin : therapeutic potential and recent advances in drug delivery. Expert opinion on investigational drugs. 2013 Aug;22(8):1063-79.

5. Boots AW, Drent M, de Boer VC, Bast A, Haenen GR. Quercetin reduces markers of oxidative stress and inflammation in sarcoidosis. Clinical nutrition. 2011 Aug;30(4):506-12.

6. Yao LH, Jiang YM, Shi J, Tomas-Barberan FA, Datta N, Singanusong R, et al. Flavonoids in food and their health benefits. Plant Foods Hum Nutr. 2004 Summer;59(3):113-22.

7. Bazzano LA, He J, Ogden LG, Loria CM, Vupputuri S, Myers L, et al. Fruit and vegetable intake and risk of cardiovascular disease in US adults: the first National Health and Nutrition Examination Survey Epidemiologic Follow-up Study. The American journal of clinical nutrition. 2002 Jul;76(1):93-9.

8. Morling JR, Yeoh SE, Kolbach DN. Rutosides for prevention of postthrombotic syndrome. Cochrane Database Syst Rev. 2013 Apr 30(4):CD005626.

9. Mozaffarian D, Benjamin EJ, Go AS, Arnett DK, Blaha MJ, Cushman M, et al. Executive Summary: Heart Disease and Stroke Statistics-2016 Update: A Report From the American Heart Association. Circulation. 2016 Jan 26;133(4):447-54.

10. Dharmashankar K, Widlansky ME. Vascular endothelial function and hypertension: insights and directions. Curr Hypertens Rep. 2010 Dec;12(6):448-55. 11. Benjamin EJ, Larson MG, Keyes MJ, Mitchell GF, Vasan RS, Keaney JF, Jr., et al. Clinical correlates and heritability of flow-mediated dilation in the community: the Framingham Heart Study. Circulation. 2004 Feb 10;109(5):613-9. 
12. Juonala M, Viikari JS, Ronnemaa T, Helenius $H$, Taittonen L, Raitakari OT. Elevated blood pressure in adolescent boys predicts endothelial dysfunction: the cardiovascular risk in young Finns study. Hypertension. 2006 Sep;48(3):424-30.

13. Shimbo D, Muntner P, Mann D, Viera AJ, Homma S, Polak JF, et al. Endothelial dysfunction and the risk of hypertension: the multi-ethnic study of atherosclerosis. Hypertension. 2010 May;55(5):1210-6.

14. Quyyumi AA, Patel RS. Endothelial dysfunction and hypertension: cause or effect? Hypertension. 2010 May;55(5):1092-4.

15. Schulz E, Gori T, Munzel T. Oxidative stress and endothelial dysfunction in hypertension. Hypertens Res. 2011 Jun;34(6):665-73.

16. Goszcz K, Deakin SJ, Duthie GG, Stewart D, Leslie SJ, Megson IL. Antioxidants in Cardiovascular Therapy: Panacea or False Hope? Front Cardiovasc Med. 2015;2:29.

17. Yang J, Guoa J, Yuanb J. In vitro antioxidant properties of rutin. LWT 2008;41:1060-6.

18. Graefe EU, Wittig J, Mueller S, Riethling AK, Uehleke B, Drewelow B, et al. Pharmacokinetics and bioavailability of quercetin glycosides in humans. J Clin Pharmacol. 2001 May;41(5):492-9.

19. Jacobs H, Moalin M, van Gisbergen MW, Bast A, van der Vijgh WJ, Haenen GR. An essential difference in the reactivity of the glutathione adducts of the structurally closely related flavonoids monoHER and quercetin. Free radical biology \& medicine. 2011 Dec 1;51(11):2118-23.

20. Lemmens KJ, Vrolijk MF, Bouwman FG, van der Vijgh WJ, Bast A, Haenen $\mathrm{GR}$. The minor structural difference between the antioxidants quercetin and 4 'Omethylquercetin has a major impact on their selective thiol toxicity. International journal of molecular sciences. 2014;15(5):7475-84.

21. Cleasby A, Yon J, Day PJ, Richardson C, Tickle IJ, Williams PA, et al. Structure of the BTB domain of Keap1 and its interaction with the triterpenoid antagonist CDDO. PLoS One. 2014;9(6):e98896.

22. Zhang DD, Hannink M. Distinct cysteine residues in Keap1 are required for Keap1-dependent ubiquitination of Nrf2 and for stabilization of Nrf2 by chemopreventive agents and oxidative stress. Mol Cell Biol. 2003 Nov;23(22):8137-51. 
23. Covas G, Marinho HS, Cyrne L, Antunes F. Activation of Nrf2 by H2O2: de novo synthesis versus nuclear translocation. Methods in enzymology. 2013;528:157-71.

24. Kobayashi A, Kang MI, Watai Y, Tong KI, Shibata T, Uchida K, et al. Oxidative and electrophilic stresses activate Nrf2 through inhibition of ubiquitination activity of Keap1. Mol Cell Biol. 2006 Jan;26(1):221-9.

25. Taguchi $\mathrm{K}$, Motohashi $\mathrm{H}$, Yamamoto $\mathrm{M}$. Molecular mechanisms of the Keap1-Nrf2 pathway in stress response and cancer evolution. Genes Cells. 2011 Feb;16(2):123-40.

26. Nguyen T, Sherratt PJ, Nioi P, Yang CS, Pickett CB. Nrf2 controls constitutive and inducible expression of ARE-driven genes through a dynamic pathway involving nucleocytoplasmic shuttling by Keap1. J Biol Chem. 2005 Sep 16;280(37):32485-92.

27. Lee JS, Surh YJ. Nrf2 as a novel molecular target for chemoprevention. Cancer Lett. 2005 Jun 28;224(2):171-84.

28. Cebula M, Schmidt EE, Arner ES. TrxR1 as a potent regulator of the Nrf2Keap1 response system. Antioxidants \& redox signaling. 2015 Oct 1;23(10):823-53. 29. Brigelius-Flohe R, Flohe L. Basic principles and emerging concepts in the redox control of transcription factors. Antioxidants \& redox signaling. 2011 Oct 15;15(8):2335-81.

30. Thirlwell C, Schulz L, Dibra H, Beck S. Suffocating cancer: hypoxiaassociated epimutations as targets for cancer therapy. Clinical epigenetics. 2011;3:9.

31. Mattson MP. Hormesis defined. Ageing research reviews. 2008 Jan;7(1):17.

32. Forman HJ, Davies KJ, Ursini F. How do nutritional antioxidants really work: nucleophilic tone and para-hormesis versus free radical scavenging in vivo. Free radical biology \& medicine. 2014 Jan;66:24-35.

33. Ursini F, Maiorino M, Forman HJ. Redox homeostasis: The Golden Mean of healthy living. Redox biology. 2016 Jan 19;8:205-15.

34. Prast-Nielsen S, Dexheimer TS, Schultz L, Stafford WC, Cheng Q, Xu J, et al. Inhibition of thioredoxin reductase 1 by porphyrins and other small molecules identified by a high-throughput screening assay. Free radical biology \& medicine. 2011 May 1;50(9):1114-23. 
35. Boesten DM, Berger A, de Cock P, Dong H, Hammock BD, den Hartog GJ, et al. Multi-targeted mechanisms underlying the endothelial protective effects of the diabetic-safe sweetener erythritol. PLoS One. 2013;8(6):e65741.

36. L'Azou B, Fernandez P, Bareille R, Beneteau M, Bourget C, Cambar J, et al. In vitro endothelial cell susceptibility to xenobiotics: comparison of three cell types. Cell Biol Toxicol. 2005 Mar;21(2):127-37.

37. Johansson K, Cebula M, Rengby O, Dreij K, Carlstrom KE, Sigmundsson K, et al. Cross Talk in HEK293 Cells Between Nrf2, HIF, and NF-kappaB Activities upon Challenges with Redox Therapeutics Characterized with Single-Cell Resolution. Antioxidants \& redox signaling. 2015 Nov 11.

38. Goedhart J, von Stetten D, Noirclerc-Savoye M, Lelimousin M, Joosen L, Hink MA, et al. Structure-guided evolution of cyan fluorescent proteins towards a quantum yield of 93\%. Nature communications. 2012;3:751.

39. Winterbourn CC. The challenges of using fluorescent probes to detect and quantify specific reactive oxygen species in living cells. Biochimica et biophysica acta. 2014 Feb;1840(2):730-8.

40. Kalyanaraman B, Darley-Usmar V, Davies KJ, Dennery PA, Forman HJ, Grisham $\mathrm{MB}$, et al. Measuring reactive oxygen and nitrogen species with fluorescent probes: challenges and limitations. Free radical biology \& medicine. 2012 Jan 01;52(1):1-6.

41. Aziza SA, Azab Mel S, El-Shall SK. Ameliorating role of rutin on oxidative stress induced by iron overload in hepatic tissue of rats. Pak J Biol Sci. 2014 Aug;17(8):964-77.

42. Lemmens KJ, van de Wier B, Vaes N, Ghosh M, van Zandvoort MA, van der Vijgh WJ, et al. The flavonoid 7-mono-O-(beta-hydroxyethyl)-rutoside is able to protect endothelial cells by a direct antioxidant effect. Toxicology in vitro : an international journal published in association with BIBRA. 2014 Jun;28(4):538-43.

43. Chen YT, Zheng RL, Jia ZJ, Ju Y. Flavonoids as superoxide scavengers and antioxidants. Free radical biology \& medicine. 1990;9(1):19-21.

44. Robak J, Gryglewski RJ. Flavonoids are scavengers of superoxide anions. Biochemical pharmacology. 1988 Mar 01;37(5):837-41.

45. Pollard SE, Kuhnle GG, Vauzour D, Vafeiadou K, Tzounis X, Whiteman M, et al. The reaction of flavonoid metabolites with peroxynitrite. Biochemical and biophysical research communications. 2006 Dec 01;350(4):960-8. 
46. Aherne SA, O'Brien NM. Protection by the flavonoids myricetin, quercetin, and rutin against hydrogen peroxide-induced DNA damage in Caco-2 and Hep G2 cells. Nutr Cancer. 1999;34(2):160-6.

47. Ruijters EJ, Haenen GR, Weseler AR, Bast A. The cocoa flavanol (-)epicatechin protects the cortisol response. Pharmacological research. 2014 Jan;79:28-33.

48. Arner ES, Holmgren A. Measurement of thioredoxin and thioredoxin reductase. Current protocols in toxicology / editorial board, Mahin D Maines. 2001 May;Chapter 7:Unit 74.

49. Livak KJ, Schmittgen TD. Analysis of relative gene expression data using real-time quantitative PCR and the 2(-Delta Delta C(T)) Method. Methods. 2001 Dec;25(4):402-8.

50. Shukla P, Lemley CO, Dubey N, Meyer AM, O'Rourke ST, Vonnahme KA. Effect of maternal nutrient restriction and melatonin supplementation from mid to late gestation on vascular reactivity of maternal and fetal placental arteries. Placenta. 2014 Jul;35(7):461-6.

51. Kauser K, Rubanyi GM. Bradykinin-induced, N omega-nitro-L-arginineinsensitive endothelium-dependent relaxation of porcine coronary arteries is not mediated by bioassayable relaxing substances. J Cardiovasc Pharmacol. 1992;20 Suppl 12:S101-4.

52. Alvares TS, Conte-Junior CA, Silva JT, Paschoalin VM. Acute L-Arginine supplementation does not increase nitric oxide production in healthy subjects. Nutr Metab (Lond). 2012 Jun 12;9(1):54.

53. Chennupati R, Meens MJ, Marion V, Janssen BJ, Lamers WH, De Mey JG, et al. Endothelial arginine resynthesis contributes to the maintenance of vasomotor function in male diabetic mice. PLoS One. 2014;9(7):e102264.

54. Mendes-Ribeiro AC, Mann GE, de Meirelles LR, Moss MB, Matsuura C, Brunini TM. The role of exercise on L-arginine nitric oxide pathway in chronic heart failure. Open Biochem J. 2009 Oct 13;3:55-65.

55. Meens MJ, Fazzi GE, van Zandvoort MA, De Mey JG. Calcitonin generelated peptide selectively relaxes contractile responses to endothelin-1 in rat mesenteric resistance arteries. J Pharmacol Exp Ther. 2009 Oct;331(1):87-95.

56. Compeer MG, Meens MJ, Hackeng TM, Neugebauer WA, Holtke C, De Mey JG. Agonist-dependent modulation of arterial endothelinA receptor function. Br J Pharmacol. 2012 Jul;166(6):1833-45. 
57. Weintraub NL, Joshi SN, Branch CA, Stephenson AH, Sprague RS, Lonigro AJ. Relaxation of porcine coronary artery to bradykinin. Role of arachidonic acid. Hypertension. 1994 Jun;23(6 Pt 2):976-81.

58. Hayward CE, Higgins L, Cowley EJ, Greenwood SL, Mills TA, Sibley CP, et al. Chorionic plate arterial function is altered in maternal obesity. Placenta. 2013 Mar;34(3):281-7.

59. Jimenez-Atienzar M, Escribano J, Cabanes J, Gandia-Herrero F, GarciaCarmona F. Oxidation of the flavonoid eriodictyol by tyrosinase. Plant physiology and biochemistry : PPB / Societe francaise de physiologie vegetale. 2005 Sep;43(9):866-73.

60. Boots AW, Kubben N, Haenen GR, Bast A. Oxidized quercetin reacts with thiols rather than with ascorbate: implication for quercetin supplementation. Biochemical and biophysical research communications. 2003 Aug 29;308(3):560-5. 61. Kuo HP, Lee DF, Xia W, Wei Y, Hung MC. TNFalpha induces HIF-1alpha expression through activation of IKKbeta. Biochemical and biophysical research communications. 2009 Nov 27;389(4):640-4.

62. Gilbert JS, Ryan MJ, LaMarca BB, Sedeek M, Murphy SR, Granger JP. Pathophysiology of hypertension during preeclampsia: linking placental ischemia with endothelial dysfunction. Am J Physiol Heart Circ Physiol. 2008 Feb;294(2):H541-50.

63. Gudmundsdottir H, Hoieggen A, Stenehjem A, Waldum B, Os I. Hypertension in women: latest findings and clinical implications. Ther Adv Chronic Dis. 2012 May;3(3):137-46.

64. Rodriguez I, Gonzalez M. Physiological mechanisms of vascular response induced by shear stress and effect of exercise in systemic and placental circulation. Front Pharmacol. 2014;5:209.

65. Ruijters EJ, Weseler AR, Kicken C, Haenen GR, Bast A. The flavanol (-)epicatechin and its metabolites protect against oxidative stress in primary endothelial cells via a direct antioxidant effect. Eur J Pharmacol. 2013 Sep 5;715(13):147-53.

66. Lemmens KJA, Sthijns MMJPE, van der Vijgh WJF, Bast A, Haenen GRMM. The antioxidant flavonoid monoHER provides efficient protection and induces the innate NRF2 mediated adaptation in endothelial cells subjected to oxidative stress. Pharmanutrition. 2014;2(3):69-74. 
67. Jaganath IB, Mullen W, Edwards CA, Crozier A. The relative contribution of the small and large intestine to the absorption and metabolism of rutin in man. Free Radic Res. 2006 Oct;40(10):1035-46.

68. Paganga G, Rice-Evans CA. The identification of flavonoids as glycosides in human plasma. FEBS Lett. 1997 Jan 13;401(1):78-82.

69. Jacobs $H$, van der Vijgh WJ, Koek GH, Draaisma GJ, Moalin $M$, van Strijdonck GP, et al. Characterization of the glutathione conjugate of the semisynthetic flavonoid monoHER. Free radical biology \& medicine. 2009 Jun 15;46(12):1567-73.

70. Jacobs $\mathrm{H}$, Koek GH, Peters R, Moalin M, Tack J, van der Vijgh WJ, et al. Differences in pharmacological activities of the antioxidant flavonoid monoHER in humans and mice are caused by variations in its metabolic profile. Clin Pharmacol Ther. 2011 Dec;90(6):852-9.

71. Zhang $X$, Song J, Shi X, Miao S, Li Y, Wen A. Absorption and metabolism characteristics of rutin in Caco-2 cells. TheScientificWorldJournal. 2013;2013:382350.

72. Nait Chabane M, Al Ahmad A, Peluso J, Muller CD, Ubeaud G. Quercetin and naringenin transport across human intestinal Caco-2 cells. The Journal of pharmacy and pharmacology. 2009 Nov;61(11):1473-83.

73. Walgren RA, Lin JT, Kinne RK, Walle T. Cellular uptake of dietary flavonoid quercetin 4'-beta-glucoside by sodium-dependent glucose transporter SGLT1. The Journal of pharmacology and experimental therapeutics. 2000 Sep;294(3):837-43.

74. Walle T, Vincent TS, Walle UK. Evidence of covalent binding of the dietary flavonoid quercetin to DNA and protein in human intestinal and hepatic cells. Biochemical pharmacology. 2003 May 15;65(10):1603-10.

75. Pickering AM, Linder RA, Zhang H, Forman HJ, Davies KJ. Nrf2-dependent induction of proteasome and $\mathrm{Pa} 28$ alphabeta regulator are required for adaptation to oxidative stress. J Biol Chem. 2012 Mar 23;287(13):10021-31.

76. Su KY, Yu CY, Chen YW, Huang YT, Chen CT, Wu HF, et al. Rutin, a flavonoid and principal component of saussurea involucrata, attenuates physical fatigue in a forced swimming mouse model. Int J Med Sci. 2014;11(5):528-37.

77. Ajit $D$, Simonyi $A$, Li $R$, Chen $Z$, Hannink $M$, Fritsche $K L$, et al. Phytochemicals and botanical extracts regulate NF-kappaB and Nrf2/ARE reporter activities in DI TNC1 astrocytes. Neurochem Int. 2016 Jul;97:49-56. 
78. Patwardhan J, Bhatt P. Flavonoids Derived from Abelmoschus esculentus Attenuates UV-B Induced Cell Damage in Human Dermal Fibroblasts Through Nrf2-ARE Pathway. Pharmacogn Mag. 2016 May;12(Suppl 2):S129-38.

79. Tian R, Yang W, Xue Q, Gao L, Huo J, Ren D, et al. Rutin ameliorates diabetic neuropathy by lowering plasma glucose and decreasing oxidative stress via Nrf2 signaling pathway in rats. European journal of pharmacology. 2016 Jan 15;771:84-92.

80. Chakrabarti S, Sinha M, Thakurta IG, Banerjee P, Chattopadhyay M. Oxidative stress and amyloid beta toxicity in Alzheimer's disease: intervention in a complex relationship by antioxidants. Curr Med Chem. 2013;20(37):4648-64.

81. Moghbelinejad S, Nassiri-AsI M, Farivar TN, Abbasi E, Sheikhi M, Taghiloo $M$, et al. Rutin activates the MAPK pathway and BDNF gene expression on betaamyloid induced neurotoxicity in rats. Toxicol Lett. 2014 Jan 03;224(1):108-13.

82. Brigelius-Flohe R. Glutathione peroxidases and redox-regulated transcription factors. Biol Chem. 2006 Oct-Nov;387(10-11):1329-35.

83. Ugusman A, Zakaria Z, Chua KH, Nordin NA, Abdullah Mahdy Z. Role of rutin on nitric oxide synthesis in human umbilical vein endothelial cells. TheScientificWorldJournal. 2014;2014:169370.

84. Knock GA, Poston L. Bradykinin-mediated relaxation of isolated maternal resistance arteries in normal pregnancy and preeclampsia. Am J Obstet Gynecol. 1996 Dec;175(6):1668-74.

85. Ahlqvist J. Atherosclerosis, and Newton, Poiseuille, Reynolds and Prandtl. Med Hypotheses. 2001 Oct;57(4):446-52.

86. Chandel NS, McClintock DS, Feliciano CE, Wood TM, Melendez JA, Rodriguez AM, et al. Reactive oxygen species generated at mitochondrial complex III stabilize hypoxia-inducible factor-1alpha during hypoxia: a mechanism of $\mathrm{O} 2$ sensing. J Biol Chem. 2000 Aug 18;275(33):25130-8.

87. Lee $\mathrm{G}$, Won HS, Lee YM, Choi JW, Oh $\mathrm{TI}$, Jang JH, et al. Oxidative Dimerization of PHD2 is Responsible for its Inactivation and Contributes to Metabolic Reprogramming via HIF-1alpha Activation. Sci Rep. 2016 Jan 07;6:18928. 88. Gloire G, Legrand-Poels S, Piette J. NF-kappaB activation by reactive oxygen species: fifteen years later. Biochemical pharmacology. 2006 Nov 30;72(11):1493-505. 
89. Kyung TW, Lee JE, Shin HH, Choi HS. Rutin inhibits osteoclast formation by decreasing reactive oxygen species and TNF-alpha by inhibiting activation of NFkappaB. Exp Mol Med. 2008 Feb 29;40(1):52-8.

90. Triantafyllou A, Mylonis I, Simos G, Bonanou S, Tsakalof A. Flavonoids induce HIF-1alpha but impair its nuclear accumulation and activity. Free radical biology \& medicine. 2008 Feb 15;44(4):657-70.

91. Tada M, Kohno M, Kasai S, Niwano Y. Generation mechanism of radical species by tyrosine-tyrosinase reaction. J Clin Biochem Nutr. 2010 Sep;47(2):162-6. 92. Klumpen E, Hoffschroer N, Zeis B, Gigengack U, Dohmen E, Paul RJ. Reactive oxygen species (ROS) and the heat stress response of Daphnia pulex: ROS-mediated activation of hypoxia-inducible factor 1 (HIF-1) and heat shock factor 1 (HSF-1) and the clustered expression of stress genes. Biol Cell. 2016 Aug 12.

93. Narayanan A, Amaya M, Voss K, Chung M, Benedict A, Sampey G, et al. Reactive oxygen species activate NFkappaB (p65) and p53 and induce apoptosis in RVFV infected liver cells. Virology. 2014 Jan 20;449:270-86.

94. Libby P. Inflammation and cardiovascular disease mechanisms. The American journal of clinical nutrition. 2006 Feb;83(2):456S-60S.

95. Larson AJ, Symons JD, Jalili T. Quercetin: A Treatment for Hypertension?-A Review of Efficacy and Mechanisms. Pharmaceuticals (Basel). 2010 Jan 19;3(1):237-50.

96. Hamalainen $M$, Nieminen $R$, Vuorela $P$, Heinonen $M$, Moilanen E. Antiinflammatory effects of flavonoids: genistein, kaempferol, quercetin, and daidzein inhibit STAT-1 and NF-kappaB activations, whereas flavone, isorhamnetin, naringenin, and pelargonidin inhibit only NF-kappaB activation along with their inhibitory effect on iNOS expression and NO production in activated macrophages. Mediators Inflamm. 2007;2007:45673.

97. Qutub AA, Popel AS. Reactive oxygen species regulate hypoxia-inducible factor 1alpha differentially in cancer and ischemia. Mol Cell Biol. 2008 Aug;28(16):5106-19.

98. Rathee P, Chaudhary H, Rathee S, Rathee D, Kumar V, Kohli K. Mechanism of action of flavonoids as anti-inflammatory agents: a review. Inflamm Allergy Drug Targets. 2009 Jul;8(3):229-35.

99. Sthijns MM, Randall MJ, Bast A, Haenen GR. Adaptation to acrolein through upregulating the protection by glutathione in human bronchial epithelial 
cells: the materialization of the hormesis concept. Biochemical and biophysical research communications. 2014 Apr 18;446(4):1029-34.

100. Shi MM, Kugelman A, Iwamoto T, Tian L, Forman HJ. Quinone-induced oxidative stress elevates glutathione and induces gamma-glutamylcysteine synthetase activity in rat lung epithelial L2 cells. J Biol Chem. 1994 Oct 21;269(42):26512-7.

101. Andrews NP, Prasad A, Quyyumi AA. N-acetylcysteine improves coronary and peripheral vascular function. Journal of the American College of Cardiology. 2001 Jan;37(1):117-23.

102. Liu T, Schroeder HJ, Wilson SM, Terry MH, Romero M, Longo LD, et al. Local and systemic vasodilatory effects of low molecular weight S-nitrosothiols. Free radical biology \& medicine. 2016 Feb;91:215-23.

103. Prasad A, Andrews NP, Padder FA, Husain M, Quyyumi AA. Glutathione reverses endothelial dysfunction and improves nitric oxide bioavailability. J Am Coll Cardiol. 1999 Aug;34(2):507-14.

104. Thengchaisri N, Hein TW, Wang W, Xu X, Li Z, Fossum TW, et al. Upregulation of arginase by $\mathrm{H} 2 \mathrm{O} 2$ impairs endothelium-dependent nitric oxidemediated dilation of coronary arterioles. Arteriosclerosis, thrombosis, and vascular biology. 2006 Sep;26(9):2035-42.

105. Rimbach G, Gohil K, Matsugo S, Moini H, Saliou C, Virgili F, et al. Induction of glutathione synthesis in human keratinocytes by Ginkgo biloba extract (EGb761). Biofactors. 2001;15(1):39-52.

106. Olaleye MT, Crown OO, Akinmoladun AC, Akindahunsi AA. Rutin and quercetin show greater efficacy than nifedipin in ameliorating hemodynamic, redox, and metabolite imbalances in sodium chloride-induced hypertensive rats. Hum Exp Toxicol. 2014 Jun;33(6):602-8. 


Chapter 6

Dual effect of acrolein on microsomal glutathione S-transferase 1 activity: effect of concentration and time.

Mireille M.J.P.E. Sthijns, Gertjan J.M. den Hartog, Caterina Scasso, Jan P. Haenen, Aalt Bast, Guido R.M.M. Haenen.

Submitted. 


\section{Abstract}

Acrolein is an $\alpha, \beta$-unsaturated aldehyde and is a main compound in diesel exhaust or cigarette smoke. Glutathione (GSH) is the major cellular protector against the damaging effects of acrolein. In addition, acrolein seems to increase the activity of the enzyme catalyzing this protective mechanism of microsomal GSH transferase 1 (MGST1) at low concentrations. In this study the effect of the concentration and the time of acrolein exposure on MGST1 activity is determined and the involvement of thiol and amino group binding in this effect is described.

Hereto, microsomes were isolated and activity was measured after incubating them with different concentrations for various incubation times with acrolein.

At concentrations lower than $5 \mathrm{mM}$, acrolein activated MGST1. Addition of GSH prevented this activation. In the first minute after addition of $5 \mathrm{mM}$ of acrolein or higher resulted in an increase in MGST1 activity, whereas after 1 minute the activity decreased.

Addition of acrolein to MGST1 at a concentration that usually stimulated MGST1 resulted in an inhibition of MGST1 when pre-exposed to $5 \mathrm{mM} \mathrm{N}$-ethylmaleimide (NEM) for 5 minutes. Addition of amino group reacting compounds resulted in an inhibition of MGST1. This reactivity towards amino groups in MGST1 can overwhelm the thiolmediated stimulation.

In conclusion, at low concentrations and short incubation times, GSH can protect against damage induced by acrolein. Increasing the concentration and incubation time first leads to an activation of MGST1 by targeting the thiol in cysteine 49. However, at higher concentrations of acrolein or at longer incubation times, next to the thiol group also amino groups in MGST1 can be targeted that results in an inhibition of MGST1. 


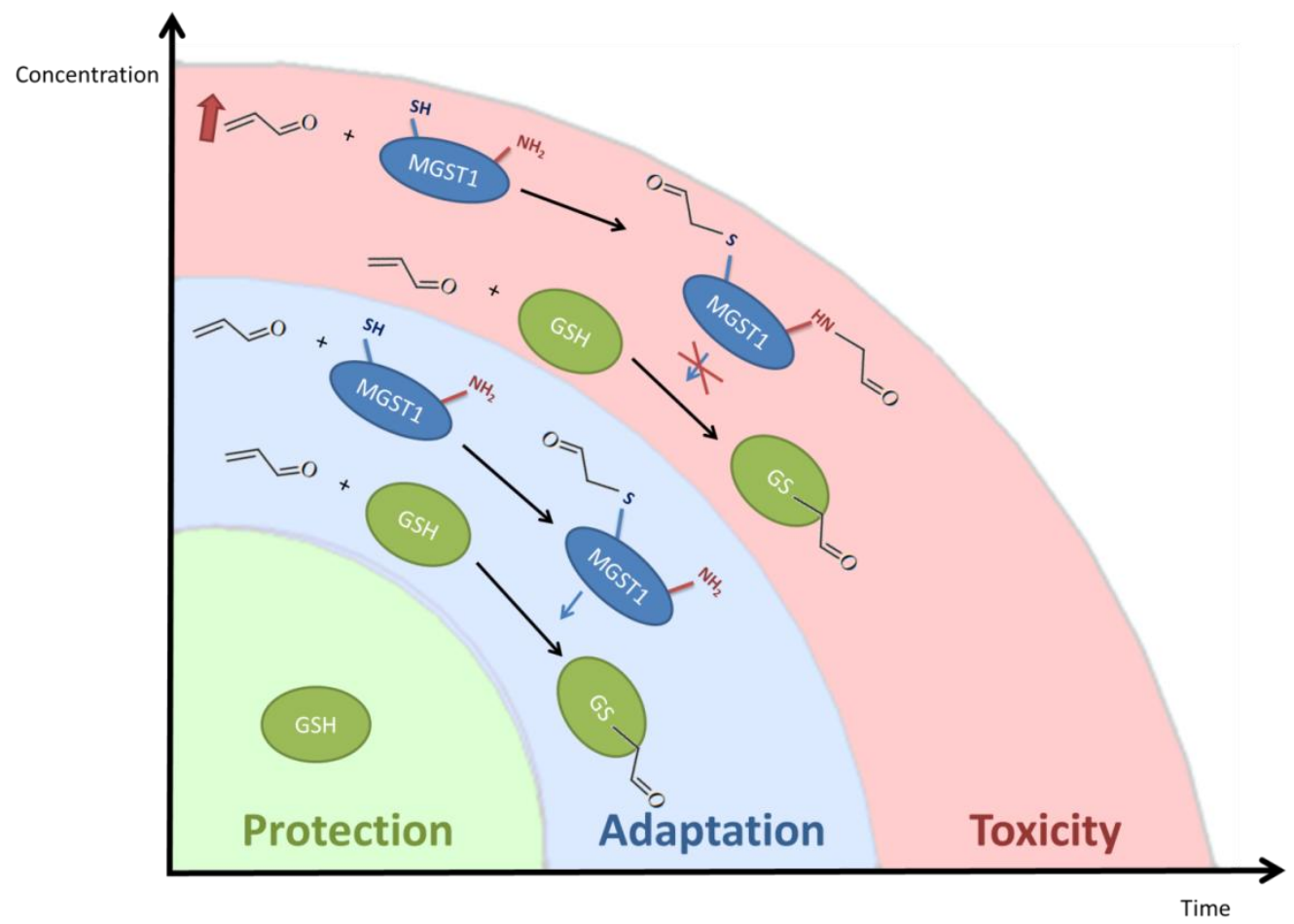




\section{Introduction}

Acrolein is an $\alpha, \beta$-unsaturated aldehyde (1). Its structure can be found in the reactive center of numerous toxic compounds, therefore acrolein can be seen as a model compound in toxicology, as a "toxicophore". Moreover, we are exposed to acrolein, because it is present in the environment e.g. in air pollution, cigarette smoke or diesel exhaust (2). In addition, it is formed endogenously in our body via biochemical processes. Acrolein prefers to react as a soft electrophile according to Hard Soft Acid Base (HSAB) principle with soft nucleophiles (3). In line with its soft character, acrolein reacts with thiols e.g. present in cysteine residues of glutathione (GSH) or (cellular) proteins via 1,4 Michael addition (4). This accounts for the toxic effect observed when living organisms are exposed to acrolein.

In the protection against endogenous and exogenous electrophiles including acrolein, GSH plays a critical role. GSH can react spontaneously with these electrophiles. The GSH transferases enhance the reaction with GSH, and therefore increase the protection $(5,6)$. GSH transferases exist in cytosolic, mitochondrial and microsomal forms $(5,6)$. Microsomal GSH transferases belong to the MAPEG (membrane-associated proteins in eicosanoid and GSH metabolism) superfamily $(5,6)$. Humans have six microsomal GSH transferase isoenzymes. Three of these are involved in drug detoxification and the others play an important role in leukotriene and prostaglandin $E$ synthesis $(5,6)$. In particular, microsomal GSH transferase 1 (MGST1) has been extensively studied. It is activated by the binding of electrophiles such as N-ethylmaleimide (NEM) (5). This is a quick direct adaptive response, since the substrate (i.e. the electrophile) directly enhances its conjugation with GSH by MGST1 and thus its detoxification (7). Cysteine 49, that is targeted by $\operatorname{NEM}(6,8)$, is located in an hydrophobic binding pocket of the enzyme, where hydrophobic electrophiles bind and this increases the GSH transferase activity of the enzyme (6). Apparently cysteine 49 works as a sensor that activates the enzyme when needed (9). Next to the cysteine, also histidine, arginine, lysine and amino groups are present in close proximity to the active site (10). It is thought that histidine residues are may involved in deprotonation of the thiol group of GSH, making GSH more reactive (10). The arginine and lysine residues are necessary for the binding to the carboxyl group of GSH (10). Electrophiles can also react with amines $(11,12)$. As described above, the consequence of the thiol reactivity of electrophiles on MGST1 is well documented. The consequences of the amino reactivity on MGST1 is largely unknown.

This prompted us to study the effect of the model compound acrolein on MGST1 in more detail. Because the effects of acrolein involve its thiol reactivity, the effects of acrolein on MGST1 are compared to that of NEM, a reference compound known to react 
with thiols. To examine the effect of the potential reaction of acrolein with amino groups, its effect is compared to that of compounds known to react with amino groups. The dependency of the effect on concentration as well as time is determined.

\section{Material and methods}

\subsection{MGST1 activity}

Isolation of microsomes was performed as previously described (13). All experiments were performed in $50 \mathrm{mM}$ sodium diphosphate buffer $\mathrm{pH} 7.4$ and at $37{ }^{\circ} \mathrm{C}$. First the different exposures were done. Then $5 \mathrm{mM}$ GSH (Sigma-Aldrich, St. Louis, MO, USA) is added. Microsomes were diluted 200-500 times in $50 \mathrm{mM}$ sodium diphosphate buffer $\mathrm{pH}$ 6.5. Then again $5 \mathrm{mM}$ GSH was added and $0.5 \mathrm{mM}$ 1-chloro-2,4-dinitrobenzene (CDNB, Sigma-Aldrich, St. Louis, MO, USA) was given. The absorbance was measured spectrophotometrically using a Cary 60 UV-visible spectrophotometer (Agilent Technologies, Santa Clara, USA) at $\lambda=340 \mathrm{~nm}$ (14).

\subsection{Exposures}

Microsomes were incubated with either 0-15 mM NEM or acrolein (SigmaAldrich, St. Louis, MO, USA) for 30 seconds after which the activity was measured.

In addition microsomes were incubated with $5 \mathrm{mM} \mathrm{NEM}$ and at 0, 0.5, 1, 3, 5 and 20 minutes the activity was measured. The same was done for acrolein. Now the activity was determined at $0,0.5,1,5,20$ and 40 minutes.

Subsequently microsomes were incubated with $5 \mathrm{mM}$ NEM and the activity was measured at $0,0.5,1,5,15,20$ and 40 minutes. Then microsomes were preincubated with $5 \mathrm{mM}$ NEM for 5 minutes, then $5 \mathrm{mM}$ acrolein was added and at the same time points the activity was determined.

MGST1 activity was determined after 30 seconds incubation with nothing (control), $5 \mathrm{mM}$ NEM or $5 \mathrm{mM}$ acrolein. Then the influence of GSH was examined by adding $5 \mathrm{mM}$ GSH before exposures to NEM or acrolein.

In order to determine whether besides thiol-reactivity acrolein also possesses reactivity towards amino $\left(\mathrm{NH}_{2}\right)$ moieties, the amino-reacting compound $5 \mathrm{mM}$ ninhydrin, 5 $\mathrm{mM}$ 1-fluoro-2,4-dinitrobenzene (FDNB) or $5 \mathrm{mM}$ phenylglyoxal (PGO) (Sigma-Aldrich, St. Louis, MO, USA) was added to the microsomes and the activity was measured at 0, 0.5, 1, 5 and 20 minutes, $0,0.5,1$ and 20 minutes or $0,0.5,5$ and 15 minutes respectively. 
Furthermore, microsomes were incubated with $5 \mathrm{mM} \mathrm{NEM}$ and the activity was measured at $0,0.5,1,5,15,20$ and 40 minutes. Then MGST1 was preincubated with $5 \mathrm{mM}$ NEM for 5 minutes, then $5 \mathrm{mM}$ ninhydrin was added and at the same time points the activity was determined. A similar experiment was repeated for either $5 \mathrm{mM}$ FDNB or 5 $\mathrm{mM}$ PGO instead of $5 \mathrm{mM}$ ninhydrin.

First $2.5 \mathrm{mM}$ acrolein was incubated with $5 \mathrm{mM}$ cysteamine (Janssen Chimica NV, Beerse, Belgium). In this case the cysteamine:acrolein ratio was set to $2: 1$. Next, $2.5 \mathrm{mM}$ acrolein was added to $0.625 \mathrm{mM}$ cysteamine, resulting in a cysteamine:acrolein ratio of 0.25:1. Both samples were measured with the High Performance Liquid Chromatography (HPLC) and Liquid Chromatography-Mass spectrometry (LC-MS) after 5 minutes and 24 hours. Also acrolein and cysteamine were determined separately with the HPLC and LCMS.

\subsection{High Performance Liquid Chromatography analysis}

HPLC analysis was performed using Agilent Technologies 1260 Infinity multidetector system (Agilent Technologies. Palo Alto, CA, USA), Agilent Technologies binary pump, and detections were carried out using Agilent technologies multiple wavelengths UV/Vis detector. An Apollo C18 column (5 $\mathrm{mm}, 15 \mathrm{~cm}$ x $4.5 \mathrm{~mm}$ ) (Grace Discovery Sciences, Bread, the Netherlands) was used. At $t=0$ and $t=5$ minutes the mobile phase contained $0.1 \%$ (v/v) trifluoroacetic acid (TFA, Sigma-Aldrich, St. Louis, MO, USA) in water. At $t=11$ minutes acetonitrile (Sigma-Aldrich, St. Louis, MO, USA) was added in a linear gradient to the mobile phase until it reaches $60 \%$. An injection volume of $10 \mu \mathrm{l}$ was used with a draw and eject speed of $1,000 \mu \mathrm{l} / \mathrm{min}$. The flow rate was set at $1 \mathrm{ml} / \mathrm{min}$ and a maximal pressure of 500 bar was applied. Before each injection a pre-injection flush cycle was done. After the injection a post injection flush cycle and 5-minute column re-equilibration was performed. The UV/Vis detector was set at wavelengths between $\lambda=200-280 \mathrm{~nm}$. Amber HPLC glass autosampler vials (GRACE, $1.5 \mathrm{ml}$ Snap ring Vials, $32 * 11.6 \mathrm{~mm}$, amber glass, $1^{\text {st }}$ hydrolytic class, wide opening, label and filling lines, Epping, Australia) were used for HPLC analysis.

\subsection{Liquid Chromatography-Mass Spectrometry}

A dilution of $1: 10$ in $98 \% \mathrm{H}_{2} \mathrm{O}, 2 \%$ acetonitrile and $0.05 \%$ TFA was used to dilute the samples. LC-MS measurements were performed using a Shimadzu Nexera UHPLC with LC-30AD pump, coupled to a DGU-20A degassing unit, CTO-20AC oven, and LCMS-2020. The column used for LC was a C18-reversed phase column (VisionHT High Load C18 column, 75- $\mu \mathrm{m}$ inner diameter x $15 \mathrm{~cm}, 1.5 \mu \mathrm{m}$ particle size) with gradient settings as shown in Table 1. An analytical flow rate of $0.3 \mathrm{ml} / \mathrm{min}$ with a stable pressure of 620 bar, 170 
injection volume of $10 \mu \mathrm{l}$ and run time of 25 minutes were applied. The mass spectrometer was set in the electrospray ionization (ESI) mode with an either positive or negative mode depending on the analyte. Settings to obtain higher-energy collisional dissociation (HCD) were put at a normalized collision energy of $25 \mathrm{eV}$. Data processing was done using Masslynx 4.1 software.

Table 1: Gradient LC-MS analysis.

\begin{tabular}{|l|l|l|}
\hline Time (minutes) & $\begin{array}{l}\text { Solvent A } \\
\mathbf{( 5 \%} \text { acetonitrile in water) }\end{array}$ & \multicolumn{1}{l|}{$\begin{array}{l}\text { Solvent B } \\
\text { (acetonitrile) }\end{array}$} \\
\hline $\mathbf{0}$ & $95 \%$ & $5 \%$ \\
\hline $\mathbf{5}$ & $95 \%$ & $5 \%$ \\
\hline $\mathbf{1 0}$ & $40 \%$ & $60 \%$ \\
\hline $\mathbf{2 0}$ & $5 \%$ & $95 \%$ \\
\hline $\mathbf{2 5}$ & $95 \%$ & $5 \%$ \\
\hline
\end{tabular}

\subsection{Statistics}

All experiments were at least performed in duplicate at $n \geq 2$ and all data are represented as mean \pm SEM. Statistical significance was determined using a t-test for independent samples with equal variances. P-values $<0.05$ were considered to be statistically significant.

\section{Results}

\subsection{Effect of the concentration of NEM and acrolein on MGST1 activity}

MGST1 is activated by thiol reactive agents. Acrolein is thiol reactive and therefore it is expected that acrolein activates the enzyme. NEM, a thiol reactive compound known to activate MGST1 was used as a positive control. It was found that up to $5 \mathrm{mM}$ of both NEM and acrolein concentration dependently activated MGST1 after a 30 second incubation (Figure $1 A$ and $B$ ). At concentrations higher than $5 \mathrm{mM}$, both NEM and acrolein inhibited MGST1 (Figure $1 \mathrm{~A}$ and $\mathrm{B}$ ). 

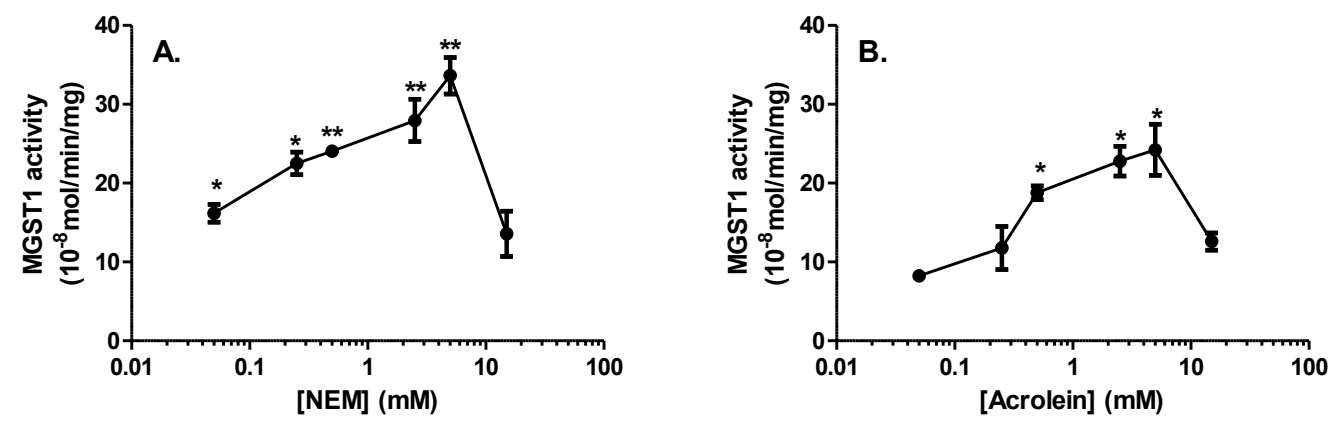

Figure 1: MGST1 is activated concentration-dependently by A) NEM or B) acrolein. Acrolein inhibits MGST1 at concentrations higher than $5 \mathrm{mM}$. Data are presented as mean \pm SEM. *P $<0.05 ;{ }^{*} \mathrm{P}<0.01$ compared to control.

\subsection{Effect of time of the NEM and acrolein exposures on MGST1 activity}

Adding $5 \mathrm{mM}$ of NEM to microsomes resulted in an increase in MGST1 activity up to 15 minutes and then the activity slightly decreased (Figure $2 \mathrm{~A}$ ). With $5 \mathrm{mM}$ acrolein the activity increased till 1 minute, after which it rapidly declined (Figure 2B).
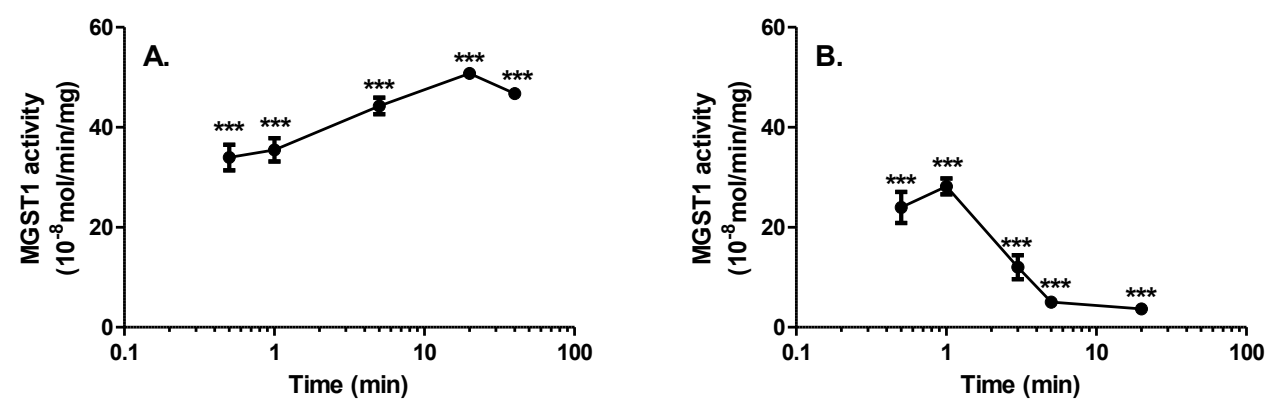

Figure 2: Over time A) $5 \mathrm{mM}$ of NEM increases MGST1 activity, whereas, B) $5 \mathrm{mM}$ of acrolein decreases MGST1 activity. Data are presented as mean \pm SEM. $* * * P<0.001$ compared to control.

\subsection{Effect of the combination of NEM and acrolein on MGST1 activity}

Apparently, NEM and acrolein stimulate MGST1, but when the concentration or incubation time increase, the enzyme can also be inactivated. For NEM it is known that activation proceeds via thiol alkylation (15). In order to further examine the dual effect of acrolein on MGST1 activity, microsomes were first incubated with NEM and subsequently 172 
with acrolein. After activation of MGST1 for 5 minutes with NEM, addition of $5 \mathrm{mM}$ acrolein results in an inhibition of the enzyme (Figure 3). Without pretreatment of NEM, 5 $\mathrm{mM}$ acrolein activates the enzyme. This indicates that the activation with NEM and acrolein are not additive; apparently the activation of the enzyme by acrolein does not proceed via a molecular mechanism that is different as that of NEM, i.e. the adduction of cysteine 49 . The inhibition of MGST1 by NEM or acrolein is apparently due to reaction with another (amino) residue.

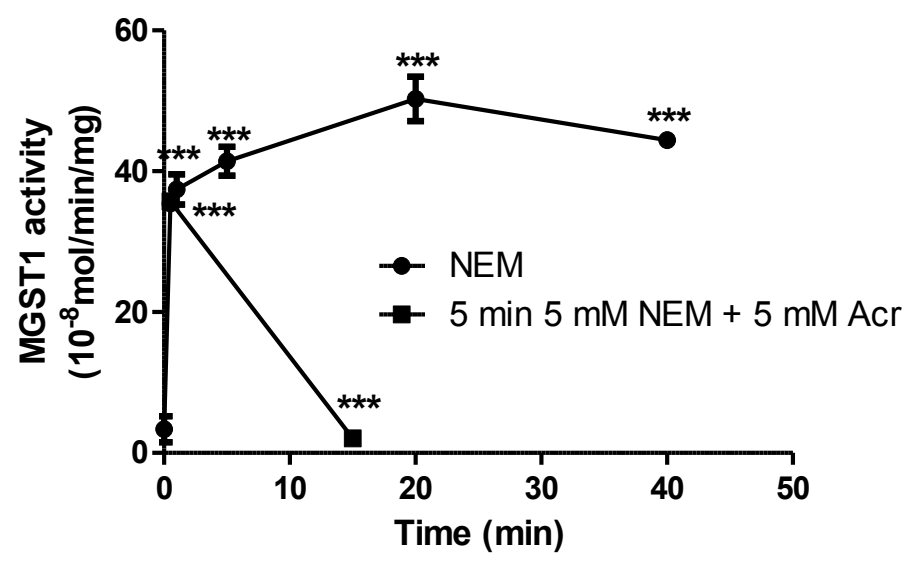

Figure 3: Incubation of microsomes with $5 \mathrm{mM}$ NEM for 5 minutes activates MGST1. Addition of $5 \mathrm{mM}$ acrolein to MGST1 pretreated with $5 \mathrm{mM}$ NEM for 5 minutes inhibits MGST1 activity. Data are presented as mean \pm SEM. $* * * \mathrm{P}<0.001$ compared to control.

\subsection{Protection by GSH}

Both NEM and acrolein can increase MGST1 activity. To further examine the role of the thiol reactivity of the compounds, the protection by GSH was determined. GSH is a thiol containing compound. Five mM GSH added before $5 \mathrm{mM}$ NEM greatly reduced the extent of activation (Figure 4). This confirms that NEM first reacts with a thiol group and that activation by NEM is due to adduction of a thiol group of MGST1. For acrolein a similar protective effect of GSH was found (Figure 4). This indicates that acrolein also first reacts with a thiol group of the enzyme that results in activation of the enzyme by acrolein. 


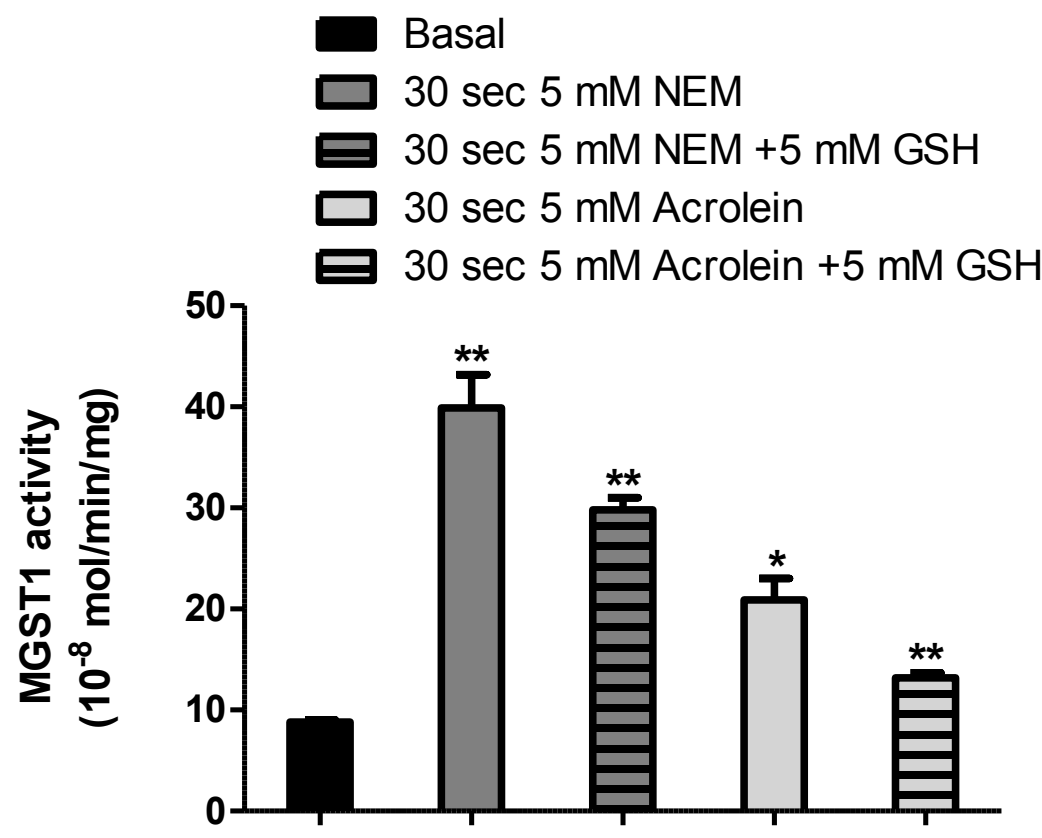

Figure 4: MGST1 activity after incubation of $5 \mathrm{mM}$ NEM/acrolein for $30 \mathrm{sec}+/-5 \mathrm{mM}$ GSH. Data are presented as mean \pm SEM. ${ }^{*} \mathrm{P}<0.05 ; * * \mathrm{P}<0.01$ compared to control.

\subsection{Effect of amino reactive compounds on MGST1 activity}

Acrolein not only reacts with thiol groups, but also with amino $\left(\mathrm{NH}_{2}\right)$ groups (11, 12). To test if $\mathrm{NH}_{2}$-adduction can explain the inhibitory effect of acrolein seen with high concentrations or long incubation times, several $\mathrm{NH}_{2}$-reactive compounds were tested. Ninhydrin, FDNB and PGO inhibit MGST1 (Figure 5). This indicates the inhibitory effect of acrolein might be attributed to its reaction with amino groups. 

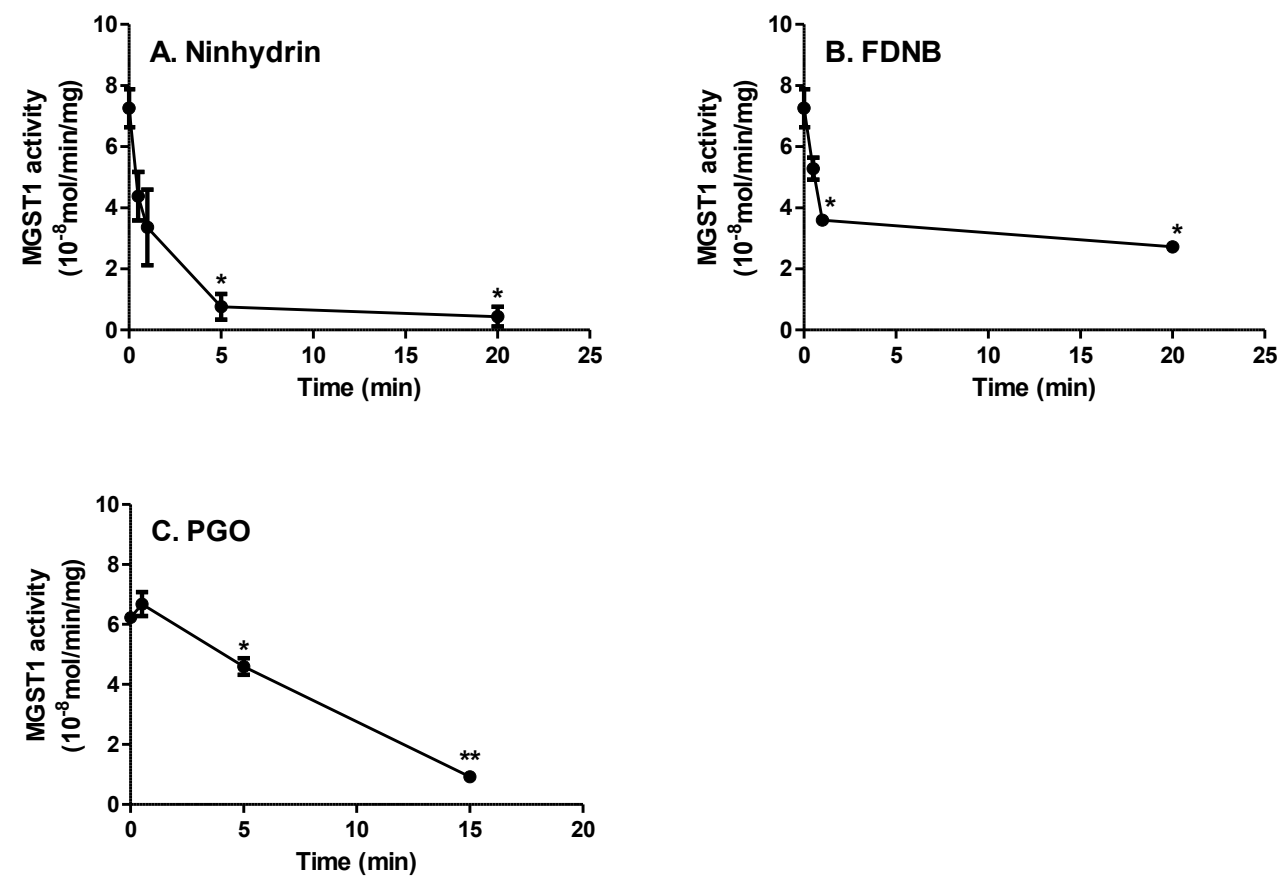

Figure 5: Inhibition of MGST1 by the amino reactive compounds A) ninhydrin, B) FDNB and PGO in time. The initial concentration was $5 \mathrm{mM}$. Data are presented as mean $\pm \mathrm{SEM}$. $* \mathrm{P}<0.05 ; * * \mathrm{P}<0.01$ compared to control.

\subsection{Effect of the combination of NEM and amino reactive compounds}

The previous experiment indicates that acrolein activates the enzyme by its reaction with the thiol group. Subsequently acrolein inactivates the enzyme by reacting with amino groups of the enzyme. The two processes were mimicked by first activation by NEM and subsequently adding the amino reactive compounds. It was found that the amino reactive compounds were indeed capable of inhibiting the NEM activated enzyme (Figure 6). This indicates that the dual effects of acrolein on the enzyme can indeed be explained by a relatively quick reaction with thiols and a relatively slow reaction with amino groups. The inhibition by adducting amino groups in MGST1 can overrule the stimulation induced by adducting thiol groups. 

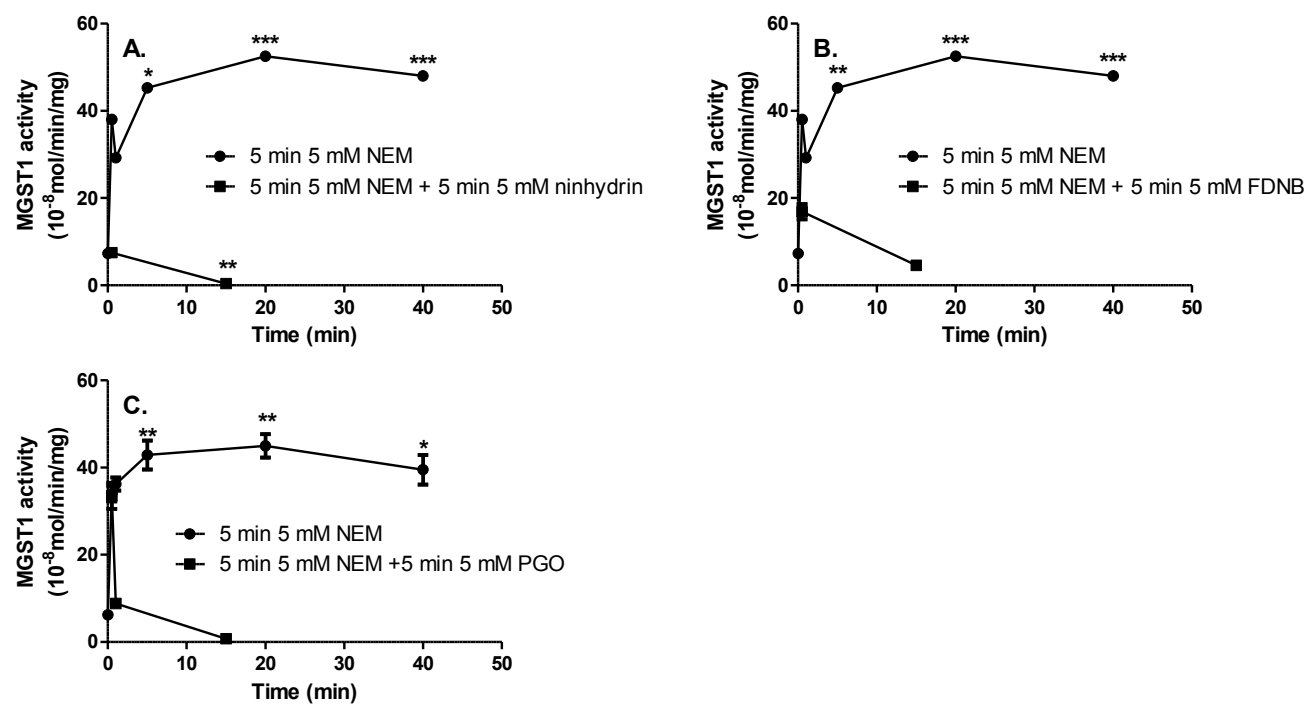

Figure 6: Incubation of microsomes for 5 minutes with $5 \mathrm{mM}$ NEM increases MGST1 activity over time. Addition of A) $5 \mathrm{mM}$ ninhydrin, B) $5 \mathrm{mM}$ FDNB and $5 \mathrm{mM}$ PGO to 5 mM NEM decreases MGST1 activity over time and prevents NEM-induced stimulation. Data are presented as mean \pm SEM. ${ }^{*} \mathrm{P}<0.05 ; * * \mathrm{P}<0.01 ; * * * \mathrm{P}<0.0001$ compared to control.

\subsection{Comparison of the amino and thiol reactivity of acrolein}

Our results show that at a low concentration and short incubation times, acrolein induced an activation of MGST1. At high concentrations and long incubation times, acrolein inhibited the enzyme. We concluded that the higher reactivity of acrolein towards thiol groups compared to amino groups explained that acrolein first induced activation and then inhibition. To put this preference in reactivity of acrolein to the test, we examined the reaction of acrolein with cysteamine, a substance that contains both a thiol and amino group. HPLC analysis and MS analysis showed that at an acrolein concentration lower than that of cysteamine, a simple adduct was formed (Table 2 and 3, Figure 7). This confirmed that, as expected, acrolein reacts with the thiol group of cysteamine via 1,4 Michael addition. At longer incubation times a new product is also detected using HPLC. Based on MS analysis this is a Schiff base, formed out of the cysteamine-acrolein adduct by an intra-molecular reaction of the carbonyl group with the amino group (Figure 7). When acrolein is present in excess compared to cysteamine, again another additional product is formed (Table 2). Using MS analysis, this was a thiozolidine derivative (Table 3 and Figure 7), formed when the excess of acrolein reacts with the cysteamine-acrolein 
adduct that was instantaneously formed. The difference in reactivity of acrolein with the thiol and amino groups of cysteamine parallels its effects on MGST1 activity. The thiol reactivity, predominantly seen at a relative low concentration and short incubation time, gives rise to activation of MGST1, which will boost protection. This can be seen as adaptation. At longer incubation times and higher concentration also the reactivity towards amino groups becomes relevant, and this inactivates MGST1. This can be seen as a response that will promote cell death.

Table 2: HPLC retention times of the reaction products of the reaction of acrolein with cysteamine in different ratios.

\begin{tabular}{|l|l|l|l|}
\hline & Peak & \\
\hline R.t.(min) & 4.3 & 9.3 & 10.3 \\
\hline Ratio Cysteamine:Acrolein 2:1 & - & +++ & - \\
\hline Ratio Cysteamine:Acrolein 0.25:1 & + & + & + \\
\hline
\end{tabular}

Table 3: Mass peaks and corresponding retention times of the reaction products of acrolein with cysteamine.

\begin{tabular}{|l|l|l|l|}
\hline \multicolumn{2}{|l|}{} & Peak & \\
\hline R.t. $(\mathbf{m i n})$ & 4.3 & 9.3 & 10.3 \\
\hline M.W. $(\mathbf{m} / \mathbf{z})$ & 133 & 192 & 116 \\
\hline M.W. $\left(\mathbf{H}^{+}\right)$ & - & - & - \\
\hline M.W. $\left(\mathbf{K}^{+}\right)$ & 172 & 231 & - \\
\hline M.W. $\left(\mathbf{N H}_{4}^{+}\right)$ & - & - & 134 \\
\hline
\end{tabular}




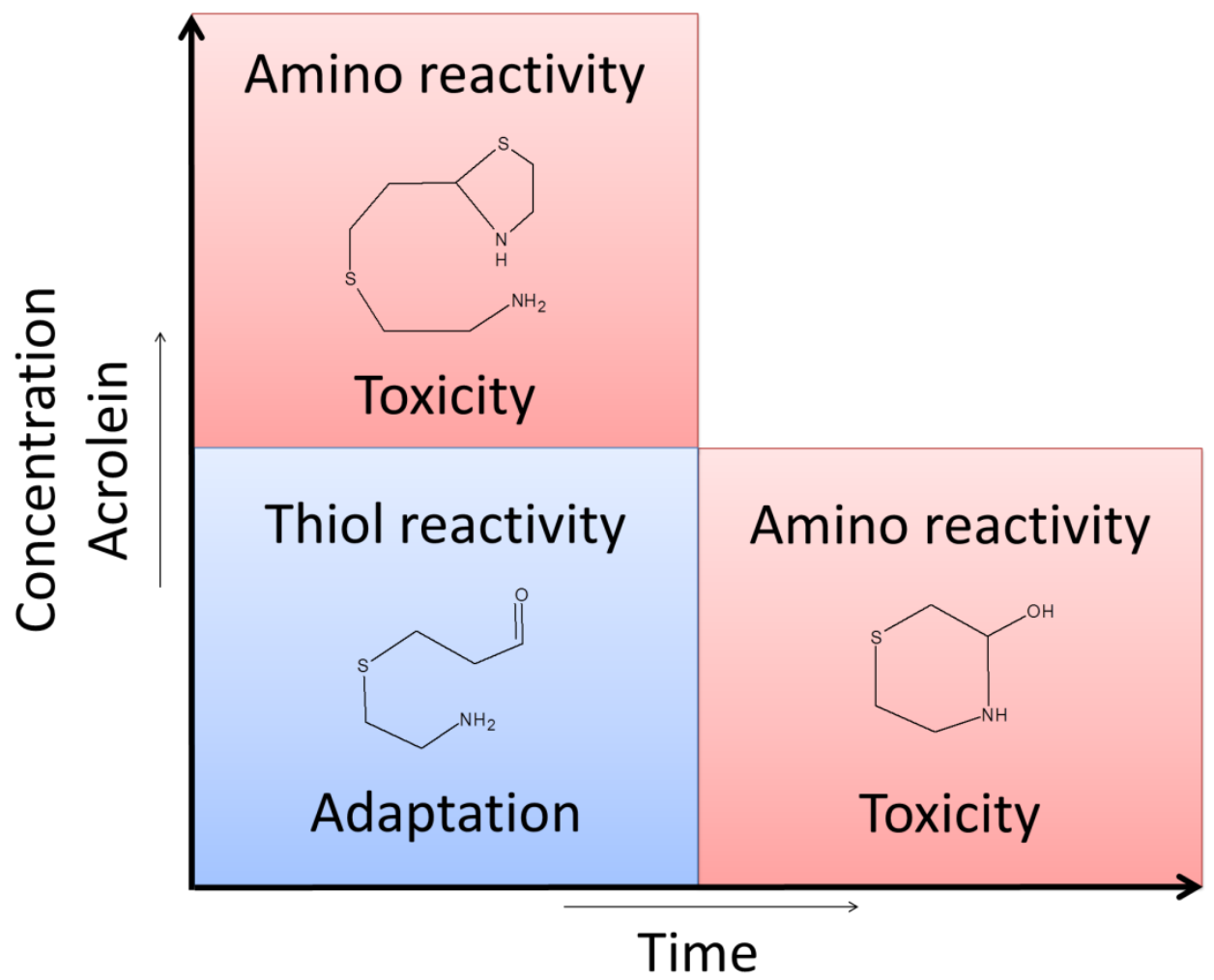

Figure 7: Reactivity of acrolein towards thiol and amino groups modeled with cysteamine. Cysteamine (2-amino-ethanethiol) contains a thiol group as well as an amino group. A fixed concentration of acrolein $(2.5 \mathrm{mM})$ is used. When acrolein is present in a relative low concentration $(2.5 \mathrm{mM})$ compared to cysteamine $(5 \mathrm{mM})$, the thiol group of cysteamine quickly reacts with acrolein in a 1,4 Michael addition to form a cysteamine-acrolein adduct. At longer incubation times ( $24 \mathrm{hrs})$ the amino group of the cysteamine-acrolein adduct reacts, a Schiff base is formed. When acrolein is present in a relative high concentration $(2.5 \mathrm{mM})$ compared to cysteamine $(0.625 \mathrm{mM})$, both the thiol and amino group react quickly with acrolein, resulting in the formation of a thiazolidine derivative next to the Schiff base. This parallels the effects of acrolein on MGST1. At a low concentration and short incubation thiol groups are adducted, causing adaptation by activation of the enzyme. At a high concentration or long incubation acrolein inactivated the enzyme promoting cell death, which can be explained by its amino reactivity. 


\section{Discussion}

NEM induces MGST1 activation by thiol alkylation $(5,6)$. Electrophiles like acrolein can activate MGST1 at concentrations lower than $5 \mathrm{mM}$. However, at concentrations higher than $5 \mathrm{mM}$ or longer incubation times, MGST1 is inhibited by acrolein. This is probably due to the finding that at high concentrations ( $>5 \mathrm{mM}$ ) next to thiol (SH) alkylation, the electrophiles also react with the amino $\left(\mathrm{NH}_{2}\right)$ groups in MGST1. The binding to the amino groups overrules thiol-mediated activation. This is also in agreement with the results using the metabolite of cysteine, the aminothiol cysteamine. At a low concentration of acrolein, acrolein prefers to react with the thiol of cysteamine, whereas at a high concentration of acrolein, next to reaction with the thiol, acrolein also reacts with the amino group of cysteamine. The thiol alkylation is an 1,4 Michael addition that results in an acrolein-cysteamine adduct, whereas amino alkylation leads to the formation either a Schiff base or a thiazolidine derivative.

Our results show that acrolein has a dual effect on MGST1 activity and that the effect depends on time as well as concentration. At low concentrations of acrolein, the cell's protective systems are activated, because acrolein increases activity of the detoxification enzyme MGST1. This can be seen as a short term adaptive process $(7,16,17)$ and the thiol group on MGST1 functions as a sensor. However, if the incubation time is too long or the concentration is too high also, the electrophile will nonspecifically react with a lot of other vital cellular targets including DNA. Then the best option is to promote cell death and get rid of the damaged cell, rather than protecting a damaged cell which could finally result in unrestrained proliferation and contribute to the development of cancer. In this response the amino group in MGST1 functions as a sensor that can switch off the enzyme and promote cell death. It has previously been found that overexpression of MGST1 will protect tumor cells against the toxic effect of anticancer drugs $(18,19)$. This confirms the protective role of MGST1 that detoxifies anticancer drugs, resulting in the establishment of anticancer drug resistance $(18,19)$. Our result indicates that targeting the amino groups of MGST1, could be a counteract of MGST1-dependent drug resistance. It has been shown that 4-methyl-ortho-benzoquinone and epigallocatechin gallate (ECGC) show a comparable concentration dependent effect on MGST1 activity as acrolein, and also GSH protects $(20,21)$. This indicates that the dual effect of acrolein also applies for other compounds. 


\section{Conclusion}

The effect of acrolein on cells consists of 3 phases: protection, adaptation and toxicity. Firstly, due to the relatively high level of the thiol GSH, GSH directly reacts with acrolein. Secondly, the cells adapt. Reaction of acrolein with the thiol group of cysteine in MGST1 will activate the enzyme and thereby the thiol group acts as a sensor. This raises the defensive shield against acrolein. Thirdly, when the exposure time is relative long or the concentration of acrolein is relative high, the cell will be damaged despite of the protection. Then, the amino groups of MGST1 function as a sensor, and its reaction with acrolein will inactivate the enzyme. This removes the protective shield and promotes the death of the damaged cell. 


\section{References}

1. Moghe A, Ghare S, Lamoreau B, Mohammad M, Barve S, McClain C, et al. Molecular mechanisms of acrolein toxicity: relevance to human disease. Toxicological sciences : an official journal of the Society of Toxicology. 2015 Feb;143(2):242-55.

2. Kehrer JP, Biswal SS. The molecular effects of acrolein. Toxicological sciences : an official journal of the Society of Toxicology. 2000 Sep;57(1):6-15.

3. LoPachin RM, Barber DS, Gavin T. Molecular mechanisms of the conjugated alpha,beta-unsaturated carbonyl derivatives: relevance to neurotoxicity and neurodegenerative diseases. Toxicological sciences : an official journal of the Society of Toxicology. 2008 Aug;104(2):235-49.

4. Witz G. Biological interactions of alpha,beta-unsaturated aldehydes. Free radical biology \& medicine. 1989;7(3):333-49.

5. Morgenstern R, Zhang J, Johansson K. Microsomal glutathione transferase 1: mechanism and functional roles. Drug metabolism reviews. 2011 May;43(2):300-6.

6. Shimoji M, Aniya Y, Morgenstern R. Activation of Microsomal Glutathione Transferase 1 in Toxicology. Boca Raton: Taylor and Francis group; 2016.

7. Sthijns MM, Weseler AR, Bast A, Haenen GR. Time in Redox Adaptation Processes: From Evolution to Hormesis. International journal of molecular sciences. 2016;17(10).

8. Busenlehner LS, Codreanu SG, Holm PJ, Bhakat P, Hebert H, Morgenstern $\mathrm{R}$, et al. Stress sensor triggers conformational response of the integral membrane protein microsomal glutathione transferase 1. Biochemistry. 2004 Sep 7;43(35):11145-52.

9. Aniya $Y$, Naito A. Oxidative stress-induced activation of microsomal glutathione S-transferase in isolated rat liver. Biochemical pharmacology. 1993 Jan 7;45(1):37-42.

10. Andersson C, Morgenstern R. Chemical modification of rat liver microsomal glutathione transferase defines residues of importance for catalytic function. Biochem J. 1990 Dec 1;272(2):479-84.

11. Tsutsui A, Tanaka K. 2,6,9-Triazabicyclo[3.3.1]nonanes as overlooked amino-modification products by acrolein. Org Biomol Chem. 2013 Nov 7;11(41):7208-11. 
12. Zhu $Q$, Sun $Z$, Jiang $Y$, Chen $F$, Wang $M$. Acrolein scavengers: reactivity, mechanism and impact on health. Molecular nutrition \& food research. 2011 Sep;55(9):1375-90.

13. Haenen GR, Bast A. Protection against lipid peroxidation by a microsomal glutathione-dependent labile factor. FEBS Lett. 1983 Aug 8;159(1-2):24-8.

14. Morgenstern R, Guthenberg C, Depierre JW. Microsomal glutathione Stransferase. Purification, initial characterization and demonstration that it is not identical to the cytosolic glutathione S-transferases A, B and C. Eur J Biochem. 1982 Nov;128(1):243-8.

15. Rinaldi R, Aniya $Y$, Svensson R, Eliasson E, Swedmark S, Shimoji M, et al. NADPH dependent activation of microsomal glutathione transferase 1. Chemicobiological interactions. 2004 Mar 15;147(2):163-72.

16. Mattson MP. Hormesis defined. Ageing research reviews. 2008 Jan;7(1):17.

17. Sthijns MM, Randall MJ, Bast A, Haenen GR. Adaptation to acrolein through upregulating the protection by glutathione in human bronchial epithelial cells: the materialization of the hormesis concept. Biochemical and biophysical research communications. 2014 Apr 18;446(4):1029-34.

18. Johansson $\mathrm{K}$, Ito $\mathrm{M}$, Schophuizen $\mathrm{CM}$, Mathew Thengumtharayil $\mathrm{S}$, Heuser VD, Zhang J, et al. Characterization of new potential anticancer drugs designed to overcome glutathione transferase mediated resistance. Molecular pharmaceutics. 2011 Oct 3;8(5):1698-708.

19. van Gisbergen MW, Cebula M, Zhang J, Ottosson-Wadlund A, Dubois L, Lambin $P$, et al. Chemical Reactivity Window Determines Prodrug Efficiency toward Glutathione Transferase Overexpressing Cancer Cells. Molecular pharmaceutics. 2016 Jun 6;13(6):2010-25.

20. Weng Z, Greenhaw J, Salminen WF, Shi Q. Mechanisms for epigallocatechin gallate induced inhibition of drug metabolizing enzymes in rat liver microsomes. Toxicology letters. 2012 Nov 15;214(3):328-38.

21. Haenen GR, Jansen FP, Vermeulen NP, Bast A. Activation of the microsomal glutathione S-transferase by metabolites of alpha-methyldopa. Archives of biochemistry and biophysics. 1991 May 15;287(1):48-52. 


Chapter 7

Redox modulation determines the fate of human melanoma cells exposed to quercetin and oxidative stress: adaptive antioxidant protection or apoptosis, a matter of life or death.

Mireille M.J.P.E. Sthijns, Antje R. Weseler, Sarah Schmidt, Elias S.J. Arnér, Katarina Johansson, Aalt Bast, Guido R.M.M. Haenen.

In progress. 


\section{Abstract}

During scavenging of radicals the antioxidant quercetin is oxidized to quercetin quinone. In most cells, quercetin quinone increases nuclear factor (erythroid-derived 2)like 2 (Nrf2)-mediated gene expression conferring antioxidant protection and adaptation to an oxidative cellular environment. Cancer cells are continuously exposed to a high level of oxidative stress, resulting in a consistently increased activity of Nrf2. If quercetin quinone enhances this activity even more this could be disadvantageous, because it may protect tumor cells against oxidative damage thereby enhancing tumor progression. The present study aims at investigating the fate of human melanoma cells exposed to quercetin and oxidative stress, a cancer cell model that is thought to facilitate intracellular generation of quercetin quinone.

Glutathione (GSH)-depleted A375 melanoma cells were exposed to quercetin and oxidative stress induced by hydrogen peroxide $\left(\mathrm{H}_{2} \mathrm{O}_{2}\right)$ or tyrosinase favoring the intracellular formation of quercetin quinone. Nrf2-mediated gene expression, thioredoxin reductase-1 (TrxR1) activity, apoptosis and necrosis, lactate dehydrogenase (LDH) leakage, glyceraldehyde-3-phosphate dehydrogenase (GAPDH) glycolytic activity, GAPDH nuclear translocation and GAPDH-mediated gene expression of apoptotic genes were analyzed.

In the presence of oxidative stress induced by $\mathrm{H}_{2} \mathrm{O}_{2}$, quercetin prevented the induction of Nrf2-mediated gene expression of heme oxygenase-1 (HMOX1) and thioredoxin reductase (TrxR) 1 (TXNRD1) and concomitant TrxR1 activity. Moreover, it was found that quercetin quinone induced apoptosis while oxidative stress induced by $\mathrm{H}_{2} \mathrm{O}_{2}$ resulted in necrosis of melanoma cells. The reduced glycolytic activity of GAPDH in the presence of oxidative stress induced by $\mathrm{H}_{2} \mathrm{O}_{2}$ was not further affected by quercetin. However, elevated nuclear translocation of GAPDH was observed which coincided with a trend towards increased ratio of pro-apoptotic versus anti-apoptotic gene expression.

In conclusion, A375 melanoma cells simultaneously exposed to high oxidative stress induced by $\mathrm{H}_{2} \mathrm{O}_{2}$ or tyrosinase and quercetin resulted in the inhibition of Nrf2mediated adaptation and shifted cell death from oxidative stress-induced necrosis to apoptosis. 


\section{HEK293 \\ (pTRAF Nrf2/HIF/NF-kB) reporter non-}

A.

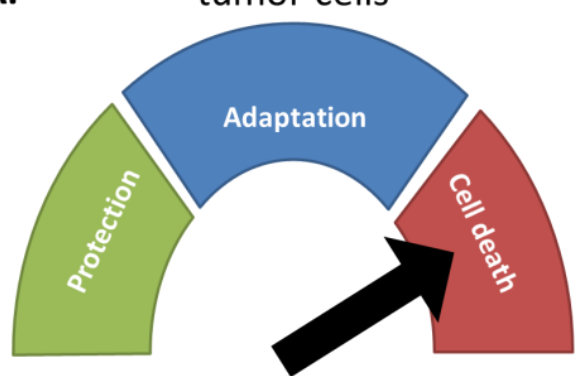

High level of oxidative stress

\section{HEK293}

(pTRAF ${ }^{\mathrm{Nrf2} / \mathrm{HIF} / \mathrm{NF}-\mathrm{kB} \text { ) }}$

$$
\text { reporter non- }
$$

C.

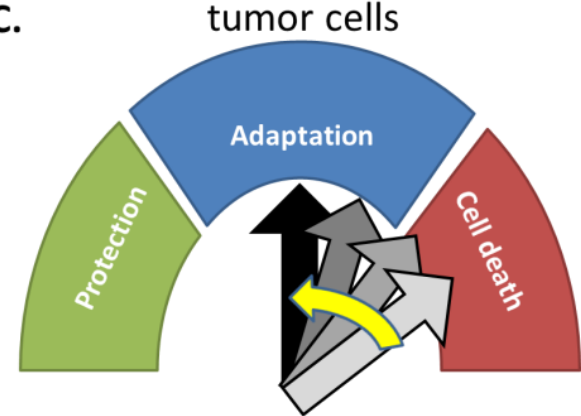

High level of oxidative

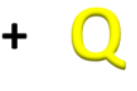

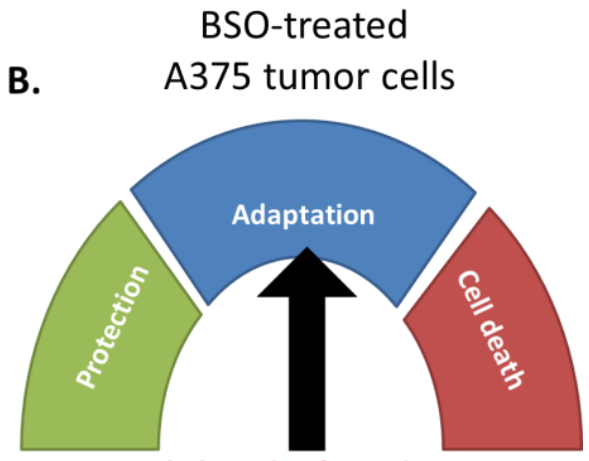

High level of oxidative stress stress

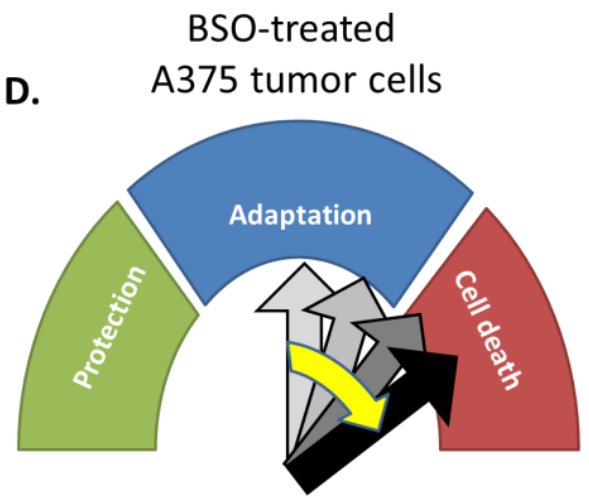

High level of oxidative stress 


\section{Introduction}

Quercetin is one of the most abundant flavonoids in our diet. It is mainly present in fruit and vegetables like apples and onions (1-11). Previous research has shown that quercetin protects against the damaging effect of reactive oxygen species (ROS). Initially this was attributed to scavenging of ROS, a direct antioxidant activity of the flavonoid (1, 12). However, direct scavenging can only partially explain the protective effects of quercetin, because of its short half-life of $17.9 \pm 2.2 \mathrm{~h}(1,13)$. There has to be an additional mechanism that may explain the long term beneficial effects.

During scavenging of ROS, quercetin is oxidized to quercetin quinone. Quercetin quinone is a soft electrophile that readily reacts with soft nucleophiles like thiols such as $\mathrm{GSH}$, the most abundant thiol-containing compound in cells (14-16). Next to GSH, quercetin quinone can also react with other thiol-containing proteins such as Kelch-like ECH-associated protein 1 (Keap1). Keap1 is an inhibitor of Nrf2 and acts as a sensor for oxidative stress and electrophiles (17). Under basal conditions Keap1 interacts with Nrf2 and induces proteosomal degradation of Nrf2. During oxidative stress or in the presence of electrophiles like flavonoid quinones, Keap1 is oxidized or adducted by these electrophiles, which will prevent proteasomal breakdown of Nrf2 (18). Nrf2 can translocate to the nucleus, where it induces an increase in Nrf2-mediated endogenous antioxidant expression, which will protect the cell against $\operatorname{ROS}(14,18,19)$. This adaptation is defined as transhormesis, since the protective shield against ROS is raised by a flavonoid quinone and not by ROS itself $(20,21)$. This means that flavonoids like quercetin do not only protect by scavenging ROS, they also protect against ROS on a longer term by inducing adaptation. The adaptation has been demonstrated in non-tumor cells (18). Tumor cells are characterized with consistently elevated ROS levels (22) This leads to a dysregulation in the redox balance, for example an consistent increase in Nrf2 response or TrxR1 activity (23). In cancer cells, increased intracellular antioxidant defense could be disadvantageous, since it may protect a tumor cell against oxidative damage and may enhance tumor progression rather than diminish unrestrained proliferation (24-28). Therefore, redox chemotherapy is promising by either increasing ROS thereby inducing damage in cancer cells resulting in cell death or decreasing the enhanced redox protection in cancer cells by targeting for example $\operatorname{Nrf2}$ or $\operatorname{TrxR1}(29,30)$. This could be achieved by using antioxidant flavonoids as adjuvants in cancer therapy, but there is a lot of controversy about that (31-44). To shed more light on the effects of quercetin on cancer cells with elevated ROS levels, the present study used a cancer cell model that favored the intracellular formation of quercetin quinone. A375 melanoma cells were chosen due to their high endogenous expression of tyrosinase which has been reported to induce radicals that probably enhance oxidative stress and catalyze the conversion of quercetin 
to quercetin quinone $(45,46)$. GSH depletion by inhibiting GSH synthesis with buthionine sulfoximine (BSO) previously performed in mouse melanoma cells B16F10, and exposure of cells to oxidative stress (47-49) further promoted the formation of quercetin quinone. This allowed to determine the fate of melanoma cells exposed to quercetin quinone, inducing either Nrf2 mediated antioxidant protection or (apoptotic) cell death.

\section{Material and Methods}

\subsection{Chemicals}

Unless stated otherwise, quercetin (2 mM; Sigma-Aldrich, St. Louis, MO, USA) was dissolved in a mixture of 1:4 $36 \mathrm{mM} \mathrm{NaOH}$ and $145 \mathrm{mM}$ potassium phosphate buffer $\mathrm{pH}=7.5$ and diluted to the appropriate concentration $(5,10,50$ or $140 \mu \mathrm{M})$ in exposure medium. Fetal bovine serum (FBS) used to culture the HEK(pTRAF ${ }^{\mathrm{Nrf} 2 / \mathrm{HIF} / \mathrm{NF}-\mathrm{KB}}$ ) cell line was derived from PAA (Pasching, Austria); and penicillin and streptomycin from Biochrom (Cambridge, United Kingdom). Eagle's minimum essential medium (EMEM) was provided by ATCC 30-2003 (Molsheim Cedex, France). FBS, penicillin, streptomycin, sodium pyruvate, all used for the A375 cells, minimal essential medium (MEM), phosphate buffered saline (PBS), RNase/DNase free water, 4-(2-hydroxyethyl)-1piperazineethanesulfonic acid (HEPES), and trypsin-EDTA were obtained from Gibco (Bleiswijk, The Netherlands). Glucose, tyrosinase, paraformaldehyde (PFA), sodium selenite, buthionine sulphoximine (BSO), horse radish peroxidase (HRP), hydrogen peroxide $\left(\mathrm{H}_{2} \mathrm{O}_{2}\right)$, isopropanol, ethanol, 5,5'-dithiobis-2-nitrobenzoic acid (DTNB), reduced $\beta$-nicotinamide adenine dinucleotide 2 '-phosphate tetrasodium hydrate (NADPH), Tris(hydroxymethyl)aminomethane hydrochloride, Trizma hydrochloride (Tris- $\mathrm{HCl}$ ), ethylenedinitrilotetraacetic acid (EDTA), bovine serum albumin (BSA), auranofin, quercetin, glutathione (GSH), phenylmethylsulfonyl fluoride, Igepal CA-630, insulin, guanidine. $\mathrm{HCl}$, Trizma base, glyceraldehyde-3-phosphate dehydrogenase from rabbit muscle (GAPDH), $\beta$ nicotinamide adenine dinucleotide hydrate $\left(N A D^{+}\right)$, glyceraldehyde-3-phosphate (G3P), potassium phosphate $\left(\mathrm{KH}_{2} \mathrm{PO}_{4}\right)$, dipotassium phosphate $\left(\mathrm{K}_{2} \mathrm{HPO}_{4}\right)$, Triton- $\mathrm{X}-100, \beta$ nicotinamide adenine dinucleotide, reduced $\beta$-nicotinamide adenine dinucleotide disodium salt hydrate $(\mathrm{NADH})$, sodium pyruvate, sodium azide $\left(\mathrm{NaN}_{3}\right)$ were derived from Sigma-Aldrich (St. Louis, MO, USA). Collagen I-coated 96-well plates (354649, BD) were delivered by Biocoat (Kgs. Lyngby, Denmark). Thermo Scientific (Bleiswijk, The Netherlands) provided DAPI. Qiazol was derived from Qiagen (Venlo, The Netherlands). iScript cDNA synthesis kit and $\mathrm{iQ}^{\mathrm{TM}} \mathrm{SYBR}^{\circledR}$ Green Supermix and Bradford reagent were purchased from Biorad (Veenendaal, The Netherlands). Recombinant selenocysteine-containing rat (Rattus norvegicus) TrxR1 was isolated like previously described (50). Arne Holmgren (Karolinska 
Institutet, Sweden) kindly provided recombinant human wild-type thioredoxin 1 (Trx1). The bicinchoninic acid (BCA; Pierce, Thermo Fisher Scientific, Etten-Leur, The Netherlands) was used to determine protein concentrations. The PE annexin V Apoptosis Detection Kit I was purchased from BD Biosciences (Breda, The Netherlands). All fluorescent labeled antibodies, CD44- allophycocyanin(APC) human, CD44- peridinin chlorophyll protein complex (PerCP)-Vio700 human and CD44- R-phycoerythrin (PE) human were derived from Miltenyi Biotec (Leiden, The Netherlands). The nuclear extract kit was obtained from ActiveMotif (La Hulpe, Belgium). The GAPDH SimpleStep ELISA kit was purchased from Abcam (Cambridge, United Kingdom).

\subsection{Quercetin quinone formation}

Either $1.6 \mathrm{nM} \mathrm{HRP}$ and $33 \mu \mathrm{M} \mathrm{H} \mathrm{O}_{2}$ or $75 \mathrm{U} / \mathrm{mL}$ tyrosinase were added to $50 \mu \mathrm{M}$ quercetin. Then an UV absorption spectrum from $\lambda=200-500 \mathrm{~nm}$ was recorded by measuring for $8 \mathrm{~min}$. every $32 \mathrm{sec}$. using the spectrophotometer. In addition, the absorption spectra from the single compounds $50 \mu \mathrm{M}$ quercetin, $1.6 \mathrm{nM} \mathrm{HRP}$ and $33 \mu \mathrm{M}$ $\mathrm{H}_{2} \mathrm{O}_{2}, 50 \mu \mathrm{M}$ quercetin and $33 \mu \mathrm{M} \mathrm{H}_{2} \mathrm{O}_{2}$, and $75 \mathrm{U} / \mathrm{mL}$ tyrosinase were determined.

\subsection{Cell cultures}

Human embryonic kidney cells (HEK293; ATCC CRL 3216) were stably transfected to the reporter cell line HEK(pTRAF ${ }^{\mathrm{Nr} 2 / \mathrm{HF} / \mathrm{NF}-\mathrm{KB}}$ ) as described before (18). Cells were cultured in EMEM supplemented with $10 \%$ (v/v) FBS, $100 \mathrm{U}$ penicillin/ml and $100 \mu \mathrm{g}$ streptomycin/ml. Cell passages of 14 - 15 were used to perform experiments.

Human epithelial malignant A375 melanoma cells (ATCC CRL-1619) were cultured in DMEM supplemented with $10 \%(\mathrm{v} / \mathrm{v}) \mathrm{FBS}, 50 \mathrm{U} / \mathrm{ml}$ penicillin and $50 \mathrm{\mu g} / \mathrm{ml}$ streptomycin, $10 \mathrm{mM}$ sodium pyruvate and $250 \mu \mathrm{M}$ glucose. Experiments were performed with cells in passage 7 - 22.

Both cell lines were incubated in a humidified atmosphere of $5 \% \mathrm{CO}_{2}$ and at $37^{\circ} \mathrm{C}$. In all experiments, MEM without any supplements and without addition of FBS was used as exposure medium. 


\subsection{Assessment of Nrf2, HIF and NF-KB activity using the HEK$\left(\right.$ PTRAF $^{\mathrm{Nrf2} / \mathrm{HIF} / \mathrm{NF}-\mathrm{kB}}$ ) reporter cell line}

Stably transfected HEK(pTRAF ${ }^{\mathrm{Nrf2/HIF/NF-KB}}$ ) were seeded in collagen I-coated 96well plates in a density of 18,000 cells/well. A quercetin $(2 \mathrm{mM})$ stock solution in ethanol (99 \% (v/v)) was prepared and diluted in MEM. Cells were exposed to either $5 \mu \mathrm{M}$ quercetin, $25 \mathrm{U}$ filtered tyrosinase or a combination of $5 \mu \mathrm{M}$ quercetin and $25 \mathrm{U}$ filtered tyrosinase. After $45 \mathrm{~min}$. at $37^{\circ} \mathrm{C}$ in the incubator the exposure medium was changed to culture medium (EMEM supplemented with $10 \%(\mathrm{v} / \mathrm{v}) \mathrm{FBS}$ and antibiotics). After $24 \mathrm{~h}$ the cells were fixed in the 96-well plates using $2 \%(\mathrm{~m} / \mathrm{v})$ ice-cold PFA. For fixing, cells were kept at room temperature for $10 \mathrm{~min}$, after which $0.02 \mu \mathrm{g} / \mathrm{ml}$ DAPI was used to stain the nuclei of the cells for $30 \mathrm{~min}$. Before storage at $4{ }^{\circ} \mathrm{C}$, PBS was added to the cells. The Operetta high-content imaging system (PerkinElmer) was used to perform analyses (51). The emission filters and exposure times to detect the fluorescent proteins, mCherry, Ypet, TFP/CFP, as well as DAPI and brightfield were described before (51), seven fields in each well were monitored. The ImageJ software was used to subtract the background applying Sternberg's rolling ball method. Further analysis was performed using the Columbus software (PerkinElmer). Individual cells were identified by the DAPI staining. The cytosolic boundaries of each cell were determined using a merged channel for all fluorescent proteins. The transcriptional activity of Nrf2, HIF and NF-KB was quantified by the intensity of mCherry, Ypet and TFP/CFP, respectively in these boundaries. Background cutoff fluorescent levels were determined using cells that contained vectors inducing a constitutive expression of the single fluorescent proteins (mCherry, Ypet and CFP) like reported earlier (51). First the cells with fluorescence intensity below the background cutoff intensity were subtracted from the signal. The remaining intensities were summed and the sum of the measured intensity of mCherry, Ypet and TFP/CFP was divided by the number of cells evaluated.

\subsection{Gene expression of heme oxygenase-1 (HMOX-1), glutamate cysteine ligase, catalytic subunit (GCLC), thioredoxin reductase-1 (TXNRD1), Tumor protein p53 (TP53), b-cell lymphoma 2 (BCL2) and caspase 8 (CASP8) in A375 cells}

A375 cells were seeded at a density of 180,000 cells/well in 6 -well plates. To reduce GSH levels, all cells were exposed to $250 \mu \mathrm{M}$ BSO in culture medium for $24 \mathrm{~h}$. Also a control was used in which the cells were not exposed to BSO. Then intracellular GSH levels were measured as explained previously $(20,52)$. Since a comparable decrease in intracellular GSH is achieved (see Supplementary Figure 1) as determined in mouse 
melanoma cells exposed to BSO (47-49), BSO-treated A375 melanoma cells were used as redox adapted cancer model. Subsequently, the BSO-treated cells were exposed to 140 $\mu \mathrm{M}$ quercetin, $100 \mu \mathrm{M} \mathrm{H}_{2} \mathrm{O}_{2}$ or a combination of $140 \mu \mathrm{M}$ quercetin and $100 \mu \mathrm{M} \mathrm{H}_{2} \mathrm{O}_{2}$ for $45 \mathrm{~min}$. Then exposure medium was replaced by culture medium (DMEM supplemented with $10 \%(\mathrm{v} / \mathrm{v}) \mathrm{FBS}, 50 \mathrm{U} / \mathrm{ml}$ penicillin combined with $50 \mu \mathrm{g} / \mathrm{ml}$ streptomycin, $10 \mathrm{mM}$ sodium pyruvate and $250 \mu \mathrm{M}$ glucose). For heme oxygenase-1, glutamate cysteine ligase, catalytic subunit, thioredoxin reductase-1, TP53, BCL2 and CASP8, RNA was isolated by chloroform/isopropanol extraction after $24 \mathrm{~h}$ of incubation. First, $500 \mu \mathrm{l}$ qiazol ${ }^{\circledR}$ was used to lyse the cells. Hundred $\mu$ l chloroform was added to extract RNA. Samples were kept at $20^{\circ} \mathrm{C}$ for $2-3 \mathrm{~min}$ to enhance extraction. Then, the samples were centrifuged for $15 \mathrm{~min}$ at $12,000 \times g$ at $4^{\circ} \mathrm{C}$. After centrifugation, the resulting upper phase was transferred to a new tube and $250 \mu \mathrm{l}$ isopropanol was added. Next, the samples were incubated overnight at $4{ }^{\circ} \mathrm{C}$, where after the centrifugation step was repeated and supernatant was removed. Subsequently, the samples were washed using $0.5 \mathrm{ml} 75 \%(\mathrm{v} / \mathrm{v})$ ethanol and centrifuged for $5 \mathrm{~min}$ at 7,500 $\times \mathrm{g}$ and $4^{\circ} \mathrm{C}$. Supernatant was discarded and pellets were kept at room temperature for $1 \mathrm{~h}$ until they were dry. The pellet was resuspended in $50 \mu \mathrm{l}$ RNase/DNase free water and incubated for $10 \mathrm{~min}$ at $60^{\circ} \mathrm{C}$. The nanodrop device (Thermo scientific nanodrop 1000 spectrophotometer, isogen life science, De Meern, The Netherlands) was used to determine the quantity of RNA. Hereafter, complementary DNA (cDNA) was made of 500 ng RNA using the iScript cDNA synthesis kit. Quantitative RT-PCR was performed in Bio Rad MyiQ Real-Time PCR Detection System (American Laboratory Trading, Boston/Cambridge) using the $\mathrm{IQ}^{\mathrm{TM}} \mathrm{SYBR}^{\circledR}$ Green Supermix (Biorad) with the following primers: heme oxygenase 1 (HMOX1; sense: 5'-CTTCTTCACCTTCCCCAACA-3' and antisense: 5'-GCTCTGGTCCTTGGTGTCAT-3'), glutamate cysteine ligase, catalytic subunit (GCLC; sense: 5'-GCACATCTACCACGCCGTC-3' and antisense: 5'-CCACCTCATCGCCCCAC-3'), thioredoxin reductase-1 (TXNRD1; sense: 5'-TCAGGGCCGTTCATTTTTAG-3' and antisense: 5'-GATCTGCCCGTTGTGTTTG-3'), TP53 (TP53; sense: 5'-TAACAGTTCCTGCATGGGCGGC-3' and antisense: 5'-AGGACAGGCACAAACACGCACC-3'), BCL2 (BCL2; sense: 5'CTGCACCTGACGCCCTTCA-3' and antisense: 5'CACATGACCCCACCGAACTCAAAGA-3') and caspase 8 (CASP8; sense: 5'-CATCCAGTCACTTTGCCAGA-3' and antisense: 5'GCATCTGTTTCCCCATGTTT- $3^{\prime}$ ). As housekeeping gene $\beta$-actin was used ( $\beta$-actin; sense: $5^{\prime}$ CCTGGCACCCAGCACAAT-3' and antisense: 5'-GCCGATCCACACGGAGTACT-3'). Relative gene expression of HMOX-1, GCLC, TXNRD1, TP53, BCL2, CASP8 gene expression was calculated by the $2^{-\triangle \Delta C T}$ method (53). The ratio between pro-apoptotic and anti-apoptotic gene expression was calculated by dividing the gene expression of the pro-apoptotic CASP8 by the anti-apoptotic BCL2. 


\subsection{NADPH-dependent DTNB reduction assay to measure activity of recombinant TrxR1}

Fifty $\mathrm{mM}$ Tris- $\mathrm{HCl} / 2 \mathrm{mM}$ EDTA buffer $(\mathrm{pH}=7.5)$ was prepared and kept at room temperature. This buffer was used to perform the direct NADPH-dependent DTNB reduction assay as previously described $(50,54)$ with minor modifications. NADPH (250 $\mu \mathrm{M})$ and BSA $(0.1 \mathrm{mg} / \mathrm{ml})$ were added to pure recombinant rat TrxR1 (150 $\mathrm{nM})$ that was incubated for $15 \mathrm{~min}$ with the following compounds: $25 \mathrm{U}$ tyrosinase, $150 \mathrm{nM}$ auranofin, $10 \mu \mathrm{M}$ quercetin, $50 \mu \mathrm{M}$ quercetin, $10 \mu \mathrm{M}$ quercetin and $25 \mathrm{U}$ tyrosinase, $50 \mu \mathrm{M}$ quercetin and $25 \mathrm{U}$ tyrosinase. During this $15 \mathrm{~min}$ the absorbance was determined using the Spectramax microplate reader (Molecular Devices) with SoftMax Pro software at $\lambda=340$ $\mathrm{nm}$. These experiments were repeated in the presence of $1 \mathrm{mM} \mathrm{GSH}$. Subsequently, the samples were diluted 20 times in the Tris-HCl/EDTA buffer $(\mathrm{pH}=7.5)$ containing $50 \mathrm{mM}$ NAPDH. Finally, the absorbance was measured at $\lambda=412 \mathrm{~nm}, 15 \mathrm{~min}$ after addition of DTNB ( $2.5 \mathrm{mM}$ dissolved in ethanol $99 \%(\mathrm{v} / \mathrm{v}))$. The data were shown as percentage of enzyme activity in treated samples compared to non-treated controls.

\subsection{Insulin coupled assay to measure TrxR1 activity in A375 cells}

A375 cells were seeded at a density of 250,000 cells/well in 6 -well plates. For the TrxR1 activity in A375 cells, the culture medium was supplemented with $12.5 \mathrm{nM}$ sodium selenite. In half of the cells GSH was depleted by adding $250 \mu \mathrm{M}$ BSO to the culture medium, which incubated for $24 \mathrm{~h}$. Subsequently, cells were exposed to either $1 \mu \mathrm{M}$ auranofin, $140 \mu \mathrm{M}$ quercetin, $200 \mu \mathrm{M} \mathrm{H}_{2} \mathrm{O}_{2}$ or the combination of $140 \mu \mathrm{M}$ quercetin and $200 \mu \mathrm{M} \mathrm{H}_{2} \mathrm{O}_{2}$ for $45 \mathrm{~min}$. After exposure, cells were lysed by adding $150 \mu \mathrm{l}$ lysis buffer containing $0.5 \mathrm{mM}$ phenylmethylsulfonyl fluoride and $1 \%(\mathrm{v} / \mathrm{v})$ Igepal CA-630 in $50 \mathrm{mM}$ Tris/2 mM EDTA buffer $(\mathrm{pH}=7.5)$. Samples were pooled and 3 rapid freeze/thaw cycles were performed. Then, the samples were centrifuged at $16,000 \mathrm{rpm}$ and $4^{\circ} \mathrm{C}$ for $30 \mathrm{~min}$. The resulting supernatant was used to determine protein concentration using the Bradford assay and measuring TrxR1 activity using the insulin coupled assay. The alternate protocol of the end-point insulin assay was applied (54). Five $\mu$ l of sample was incubated in the presence of $80 \mathrm{mM}$ HEPES buffer, $275 \mu \mathrm{M}$ insulin, $1.298 \mu \mathrm{M}$ NAPDH, $12.5 \mathrm{mM}$ EDTA and $20 \mu \mathrm{M} \operatorname{Tr} x 1$ at $37^{\circ} \mathrm{C}$ for $40 \mathrm{~min}$. DTNB was diluted in $7.2 \mathrm{M}$ guanidine- $\mathrm{HCl}$ to a concentration of $1 \mathrm{mM}$. After addition of $200 \mu \mathrm{l} 7.2 \mathrm{M}$ guanidine- $\mathrm{HCl}$ containing $1 \mathrm{mM}$ DTNB, the absorbance was measured at $\lambda=412 \mathrm{~nm}$ using a Spectramax microplate reader (Molecular Devices). The data were shown as percentage of enzyme activity in treated samples compared to non-treated controls. 


\subsection{GAPDH activity in A375 cells}

A375 cells were seeded at a density of 180,000 cells/well in 6-well plates. Then, cells were exposed to $250 \mu \mathrm{M}$ of BSO in culture medium for $24 \mathrm{~h}$. Subsequently, cells were exposed to either $200 \mu \mathrm{M} \mathrm{H}_{2} \mathrm{O}_{2}, 140 \mu \mathrm{M}$ quercetin or the combination of $200 \mu \mathrm{M} \mathrm{H}_{2} \mathrm{O}_{2}$ and $140 \mu \mathrm{M}$ quercetin. After $45 \mathrm{~min}$ of exposure, the cells were washed with PBS and $500 \mu \mathrm{l}$ lysis buffer ( $0.1 \mathrm{M}$ potassium phosphate buffer containing $10 \mathrm{mM}$ EDTA disodium salt, $\mathrm{pH}$ $=7.5$ and $1 \%(\mathrm{v} / \mathrm{v})$ Triton-X-100) was added. After $30 \mathrm{~min}$ of incubation on ice, cells were scraped and lysates were transferred to eppendorf tubes. To remove cellular debris, the samples were centrifuged at $14,000 \mathrm{rpm}$ and $4^{\circ} \mathrm{C}$ for $10 \mathrm{~min}$. Then, the supernatant was stored at $-80^{\circ} \mathrm{C}$. The BCA assay was used to determine the protein concentration, while GAPDH activity was measured as previously described (55). In the supplementary material and methods the GAPDH activity measurement on isolated protein was described.

\subsection{Detection of cytotoxicity using the lactate dehydrogenase (LDH) assay}

A375 cells were exposed to BSO first before either $200 \mu \mathrm{M} \mathrm{H} \mathrm{H}_{2}, 140 \mu \mathrm{M}$ quercetin or the combination of $200 \mu \mathrm{M} \mathrm{H}_{2} \mathrm{O}_{2}$ and $140 \mu \mathrm{M}$ quercetin were added as delineated in the paragraph 'GAPDH activity in A375 cells'. The exposure media were replaced by culture medium (DMEM supplemented with $10 \%$ (v/v) FBS, $50 \mathrm{U} / \mathrm{ml}$ penicillin combined with $50 \mu \mathrm{g} / \mathrm{ml}$ streptomycin, $10 \mathrm{mM}$ sodium pyruvate and $250 \mu \mathrm{M}$ glucose). After $24 \mathrm{~h}$ incubation the culture medium was collected for measuring LDH activity. Fifty $\mu \mathrm{l}$ of medium collected from each well was pipetted into a 96-well plate. Then, $50 \mu \mathrm{l}$ of a solution containing $0.25 \mathrm{mg} / \mathrm{ml} \mathrm{NADH}$ in $100 \mathrm{mM}$ sodium pyruvate (dissolved in $500 \mathrm{mM}$ potassium phosphate buffer, $\mathrm{pH}=7.5$ ) was added, after which the absorbance was determined at $\lambda=340 \mathrm{~nm}$ for $4 \mathrm{~min}$ using the Spectramax microplate reader (Molecular Devices). Cytotoxicity was calculated as a percentage of the LDH activity induced by $3 \%$ (v/v) Triton-X-100 for $15 \mathrm{~min}$ (set to $100 \%$ cytotoxicity).

\subsection{Nuclear and cytosolic extraction and nuclear translocation of GAPDH in $\mathrm{A} 375$ cells}

A375 cells were seeded at a density of 1,700,000 cells/well in $100 \mathrm{~mm}$ dishes. Then half of the dishes were exposed to $250 \mu \mathrm{M}$ BSO in culture medium for $24 \mathrm{~h}$ to deplete GSH. Subsequently, cells were exposed to either $200 \mu \mathrm{M} \mathrm{H}_{2} \mathrm{O}_{2}, 140 \mu \mathrm{M}$ quercetin or the combination of $200 \mu \mathrm{M} \mathrm{H}_{2} \mathrm{O}_{2}$ and $140 \mu \mathrm{M}$ quercetin in MEM for $45 \mathrm{~min}$. Then medium was exchanged to cell culture medium and the cells were left in the incubator overnight. Next, the nuclear extract kit from Active Motif was used to make nuclear 
extracts. Protein concentrations were determined by the Bradford assay. Finally, the nuclear presence of GAPDH was examined with the GAPDH SimpleStep ELISA kit.

\subsection{Flow cytometry to determine apoptosis and necrosis of A375 cells}

A375 cells were exposed as described in the paragraph 'GAPDH activity in A375 cells'. After cell exposure the medium was changed to culture medium (DMEM supplemented with $10 \%$ (v/v) FBS, $50 \mathrm{U} / \mathrm{ml}$ penicillin and $50 \mu \mathrm{g} / \mathrm{ml}$ streptomycin, $10 \mathrm{mM}$ sodium pyruvate and $250 \mu \mathrm{M}$ glucose) and the cells were incubated at $37^{\circ} \mathrm{C}$ for $24 \mathrm{~h}$. Scraped cells were used as a positive control for necrosis. Subsequently, cells were trypsinized by adding $200 \mu \mathrm{l} 0.05 \%(\mathrm{v} / \mathrm{v})$ trypsin-EDTA to each well. After incubating the cells at $37^{\circ} \mathrm{C}$ for $5 \mathrm{~min}, 800 \mu \mathrm{l}$ culture medium was added. Then, the PE annexin $V$ Apoptosis Detection Kit was used to determine the amount of apoptotic and necrotic cells in the samples. Cells were counted and approximately 500,000 cells/well were added to a 96-well plate. Next, $200 \mu$ l fluorescence-activated cell sorting (FACS) buffer (PBS ( $\mathrm{pH}=7.2$ ) containing $\left.1 \%(\mathrm{v} / \mathrm{v}) \mathrm{FBS}, 0.02 \%(\mathrm{~m} / \mathrm{v}) \mathrm{NaN}_{3}\right)$ was added to each well. Then, the plate was centrifuged for $3 \mathrm{~min}$ at $2,000 \mathrm{rpm}$ and $20^{\circ} \mathrm{C}$. After removing the supernatant, the plate was vortexed and the samples were washed twice with PBS. Then $500 \mu \mathrm{l} 1 \mathrm{x}$ binding buffer containing $0.1 \mathrm{M}$ Hepes/ $\mathrm{NaOH}(\mathrm{pH} 7.4), 1.4 \mathrm{M} \mathrm{NaCl}, 25 \mathrm{mM} \mathrm{CaCl}_{2}$ diluted 1:10 in distilled water was used to resuspend the cells. Hundred $\mu$ cell suspension was transferred to a FACS tube (BD Falcon round-bottom tube), where $5 \mu$ of both PE-labeled annexin $V$ and 7amino-actinomycin (7-AAD) were added. After an incubation of $15 \mathrm{~min}$ in the dark at room temperature, $400 \mu \mathrm{l}$ of $1 \mathrm{x}$ binding buffer was added. The samples were analyzed by a FACSCanto II with the FACSDiva software (BD Biosciences, Erembodegem, Belgium). To compensate for fluorophore spill-over, APC, PerCP-Vio700 and PE labelled anti-human CD44 antibodies were used and the spill-over was calculated and subtracted from the samples measured.

\subsection{Statistics}

All experiments were at least performed in duplicate and repeated at least 3 times. All data were presented as mean \pm SEM. Statistically significant differences between two test conditions were assessed by independent samples Student's t-tests assuming normal distribution of the data. P-values $<0.05$ were considered as statistically significant. 


\section{Results}

\subsection{In situ formation of quercetin quinone}

Quercetin had a specific UV/VIS absorption spectrum in the wavelength range of $\lambda=200-500 \mathrm{~nm}$ (Figure 1A). Within $8 \mathrm{~min}$ no change in the absorption spectrum of quercetin was seen, indicating that the rate of spontaneous oxidation was neglectible at $\mathrm{pH}=7.4$. Spectra of the combination of either $\mathrm{HRP}$ and $\mathrm{H}_{2} \mathrm{O}_{2}$ or tyrosinase in the absence of quercetin indicated that the absorption of $\mathrm{HRP}$ and $\mathrm{H}_{2} \mathrm{O}_{2}$ or tyrosinase in solution was neglectible at the wavelengths $(\lambda=200-500 \mathrm{~nm})$ analyzed.
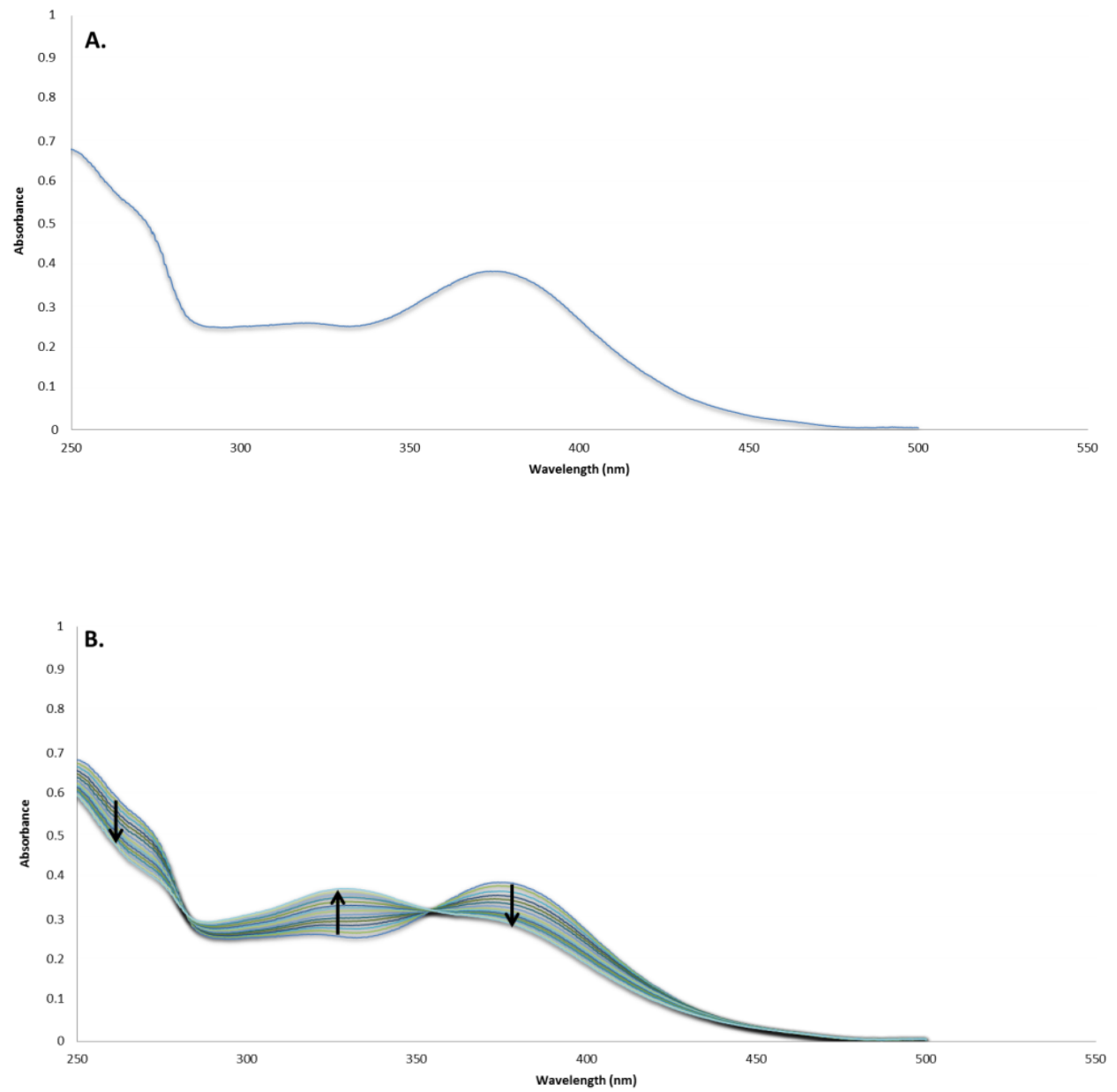


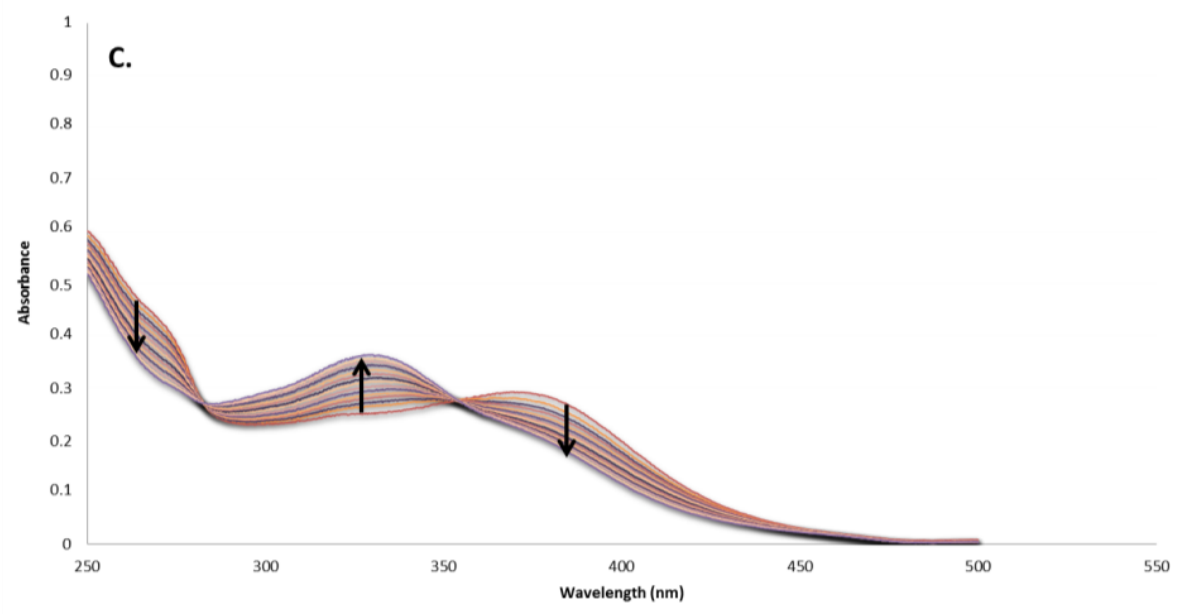

D.
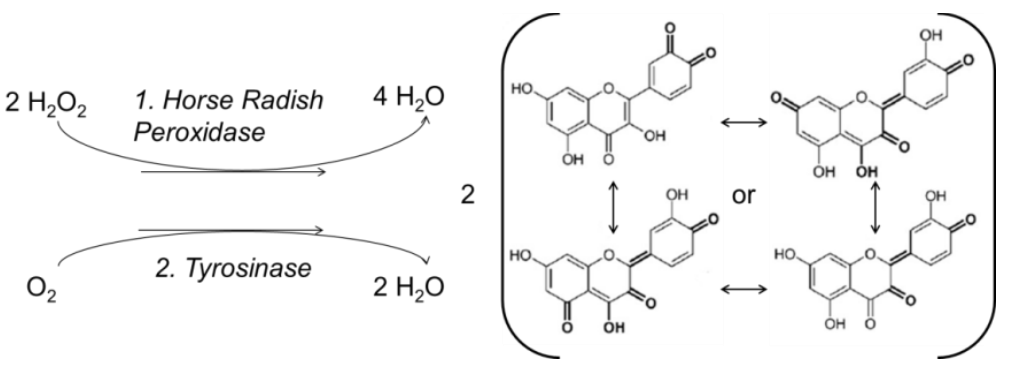

Figure 1: UV scans of $50 \mu \mathrm{M}$ quercetin (A), quercetin quinone generated by adding 1.6 nM HRP and $33 \mu \mathrm{M} \mathrm{H}_{2} \mathrm{O}_{2}$ to $50 \mu \mathrm{M}$ quercetin (B) and quercetin quinone generated by 75 $\mathrm{U} / \mathrm{ml}$ tyrosinase (C). HRP and $\mathrm{H}_{2} \mathrm{O}_{2}$ alone or tyrosinase alone did not give an absorption spectrum. After starting the reaction with addition of quercetin, a UV scan was made every 32 seconds for $8 \mathrm{~min}$. Reaction scheme of the oxidation of quercetin to quercetin quinone by either HRP and $\mathrm{H}_{2} \mathrm{O}_{2}$ or tyrosinase; quercetin quinone exists in 4 tautomers (D).

Addition of either the combination of $\mathrm{HRP}(1.6 \mathrm{nM})$ and $\mathrm{H}_{2} \mathrm{O}_{2}(33 \mu \mathrm{M}$; Figure 1B) or tyrosinase $(75 \mathrm{U} / \mathrm{ml}$; Figure $1 \mathrm{C})$ to quercetin $(50 \mu \mathrm{M})$ decreased the absorbance at a wavelength of $\lambda=375 \mathrm{~nm}$ by approximately $25 \%$ after 8 min with isosbestic points at 282 and $355 \mathrm{~nm}(14,15)$. This indicated that both, the combination of $\mathrm{HRP}$ and $\mathrm{H}_{2} \mathrm{O}_{2}$ and tyrosinase oxidized quercetin to quercetin quinone (Figure 1D). In cells exposed to quercetin, extracellular addition of either $\mathrm{H}_{2} \mathrm{O}_{2}$ or tyrosinase has also been shown to induce the formation of radicals that lead to oxidative stress $(46,56)$ that could lead to the formation of quercetin quinone $(14,57-59)$. 


\subsection{Quercetin quinone decreased NF-KB and HIF activity and increased Nrf2 activity in stable HEK(pTRAF ${ }^{\mathrm{Nrf2} / \mathrm{HIF} / \mathrm{NF}-\mathrm{KB}}$ ) reporter cells}

To investigate the effect of quercetin on the redox sensitive transcription factors Nrf2, NF-KB and HIF in a non-tumor cell line, HEK(pTRAF $\left.{ }^{\mathrm{Nr} 2 / \mathrm{HIF} / \mathrm{NF}-\mathrm{kB}}\right)$ reporter cells were used, stably expressing a vector containing response elements for Nrf2, HIF and NF-KB coupled to three different fluorescent proteins. Stable expression of the vector in HEK293 cells was previously verified with Nrf2 inducer tert-butylhydroquinone (tBHQ), NF-KB inducer tumor necrosis factor $\alpha$ (TNF $\alpha$ ) and HIF inducer dimethyloxaloylglycine (DMOG) (18). The effect of the various test conditions on cell number was shown in the supplementary results (Supplementary Figure 2). Incubation with quercetin in the absence of $25 \mathrm{U}$ tyrosinase (to induce oxidative stress) for $45 \mathrm{~min}$, enhanced Nrf2 activity after $24 \mathrm{~h}$ and did not affect the activity of the transcription factors NF-KB and HIF (Figure 2). Twenty-five $U$ tyrosinase to induce oxidative stress increased the activity of all three transcription factors (Figure 2). Quercetin quinone generated by the simultaneous exposure to quercetin and tyrosinase, reduced NF-KB and HIF activity (Figure $2 \mathrm{~B}$ and $\mathrm{C}$ ) and elevated Nrf2 activity compared to $25 \mathrm{U}$ tyrosinase alone (Figure $2 \mathrm{~A}$ ).
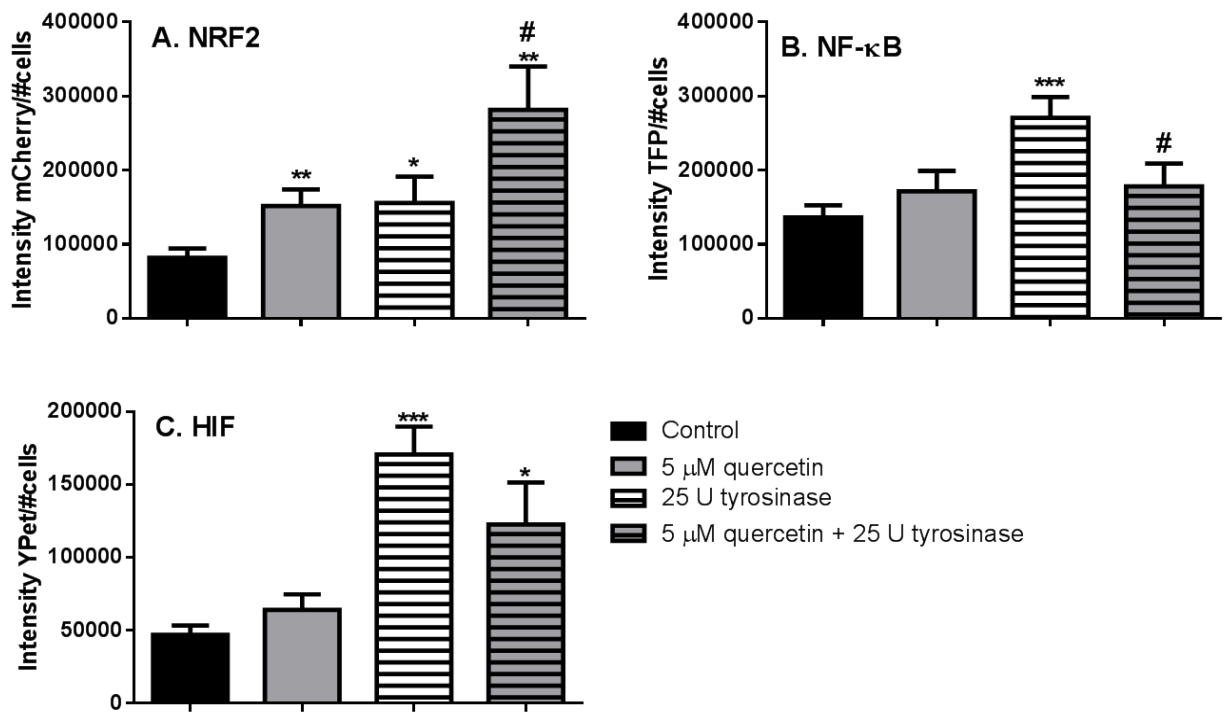

Figure 2: Nrf2, HIF and NF-KB activity in a stable HEK(pTRAF $\left.{ }^{N r f 2 / H I F / N F-K B}\right)$ reporter cell line exposed to indicated conditions for $24 \mathrm{~h}$. Twenty-five $\mathrm{U}$ tyrosinase was used to induce oxidative stress, while quercetin quinone was produced by adding $25 \mathrm{U}$ tyrosinase to 5 $\mu \mathrm{M}$ quercetin. A) mCherry intensity increased by addition of either quercetin or tyrosinase. Quercetin quinone increased mCherry intensity compared to the mCherry intensity measured after exposure to tyrosinase alone. B) TFP intensity was increased by 198 
tyrosinase, while quercetin quinone induced a decrease compared to the TFP intensity induced by tyrosinase. D) YPet intensity increased due to tyrosinase, whereas quercetin quinone showed less YPet intensity compared to tyrosinase alone. For Nf-KB and HIF transcription factor activities no effect was seen for quercetin alone. All intensities were corrected for the number of cells measured. $\mathrm{N}=3$ and data are presented as mean \pm SEM. ${ }^{*} \mathrm{P}<0.05 ;{ }^{* * \mathrm{P}}<0.01 ;{ }^{* * * \mathrm{P}}<0.001$ compared to control, \#P<0.05 compared to the condition of $25 \mathrm{U}$ tyrosinase to induce oxidative stress.

\subsection{Quercetin quinone decreased the expression of endogenous antioxidant genes HMOX-1, GCLC and TXNRD1 induced by $\mathrm{H}_{2} \mathrm{O}_{2}$ in BSO- treated melanoma cells ( $\mathrm{A} 375$ cell line)}

To investigate the effects of quercetin quinone on the transcriptional activity of Nrf2 in BSO-treated A375 melanoma cells expression of the Nrf2-regulated endogenous antioxidant genes HMOX-1, GCLC and TXNRD1 was measured after $24 \mathrm{~h}$ exposure to quercetin in the presence of $200 \mu \mathrm{M} \mathrm{H} \mathrm{O}_{2}$. Quercetin alone did not affect the expression of all 3 genes compared to control (Figure 3). $\mathrm{H}_{2} \mathrm{O}_{2}$ increased the expression of HMOX-1 (39.6 \pm 8.6 fold), GCLC ( $2.5 \pm 0.6$ fold) and TXNRD1 (3.5 \pm 0.7 fold) compared to control (Figure 3). Quercetin in the presence of $\mathrm{H}_{2} \mathrm{O}_{2}$ did not alter $\mathrm{GCLC}$ expression (Figure 3B), but resulted in a significantly lower expression of HMOX-1 (23.3 \pm 9.4 fold) and TXNRD1 (1.7 \pm 0.8 fold) induced by $\mathrm{H}_{2} \mathrm{O}_{2}$ (Figure $3 \mathrm{~A}$ and $\mathrm{C}$ ). 

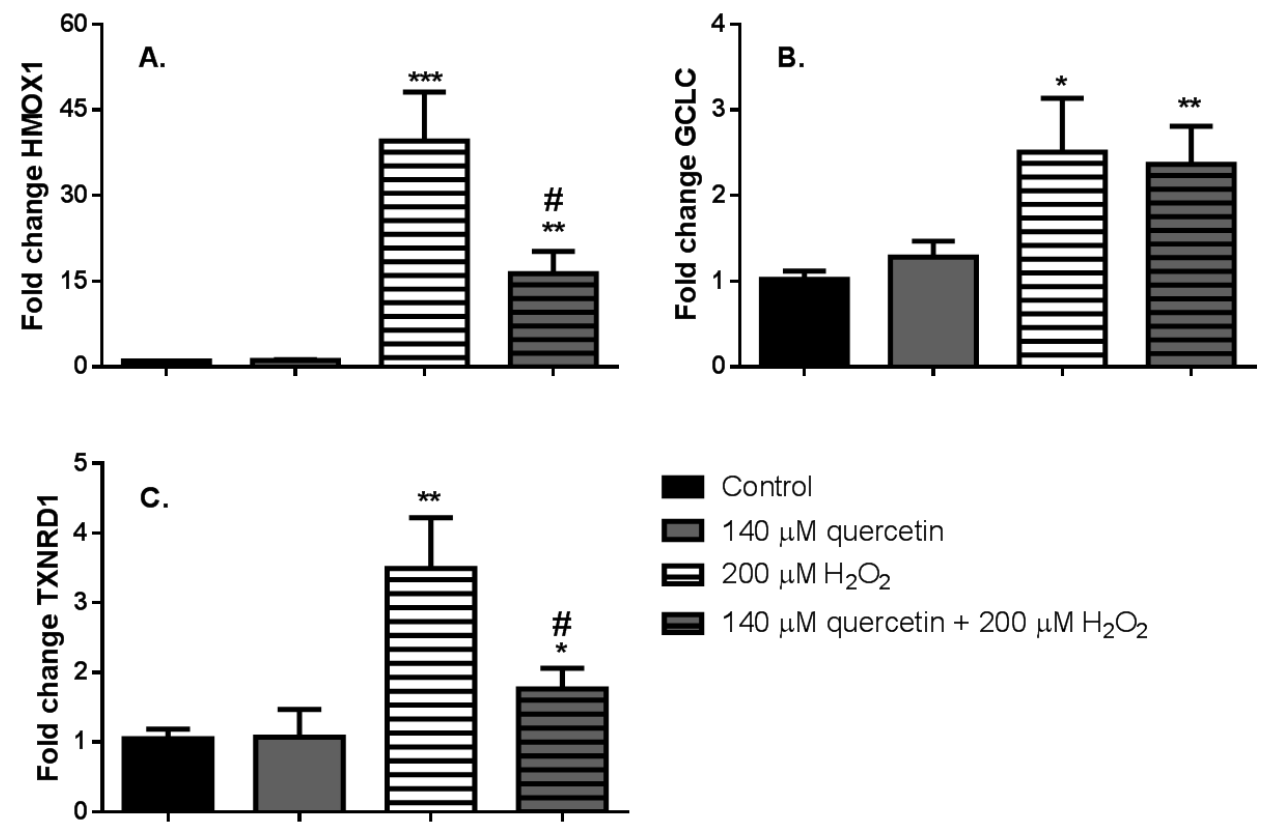

Figure 3: mRNA expression of antioxidant genes heme oxygenase-1 (HMOX1), glutamate cysteine ligase, catalytic subunit (GCLC) and thioredoxin reductase 1 (TXNRD1) in BSOtreated A375 cells exposed to indicated conditions for $24 \mathrm{~h}$. Solely quercetin (140 $\mu \mathrm{M})$ had no effect on the measured endogenous antioxidant genes. Twohundred $\mu \mathrm{M}_{2} \mathrm{O}_{2}$ induced the mRNA expression of HMOX1 (A), GCLC (B) and TXNRD1 (C). However, quercetin quinone (generated by the combination of $200 \mu \mathrm{M} \mathrm{H}_{2} \mathrm{O}_{2}$ and $140 \mu \mathrm{M}$ quercetin) induced a decrease in HMOX1 (A) and TXNRD1 (B) mRNA expression compared to the HMOX1 and TXRND1 MRNA expression induced by $200 \mu \mathrm{M} \mathrm{H}_{2} \mathrm{O}_{2}$, whereas quercetin quinone had no effect on the GCLC MRNA expression compared to the GCLC expression induced by $200 \mu \mathrm{M} \mathrm{H}_{2} \mathrm{O}_{2}$. Twohundred $\mu \mathrm{M} \mathrm{H}_{2} \mathrm{O}_{2}$ was used to induce oxidative stress, while quercetin quinone was produced by adding $200 \mu \mathrm{M} \mathrm{H} \mathrm{O}_{2}$ to $140 \mu \mathrm{M}$ quercetin. $\mathrm{N}=3$ and data are presented as mean $\pm \mathrm{SEM}$. ${ }^{*} \mathrm{P}<0.05 ;{ }^{*} \mathrm{P}<0.01 ; * * * \mathrm{P}<0.001$ compared to control. \#P<0.05 compared to the condition of $200 \mu \mathrm{M} \mathrm{H}_{2} \mathrm{O}_{2}$ to induce oxidative stress.

\subsection{Quercetin quinone inhibited TrxR1 activity, which could be prevented by GSH}

Since quercetin quinone appeared to attenuate the upregulation of TXNRD1 expression in the presence of $200 \mu \mathrm{M} \mathrm{H}_{2} \mathrm{O}_{2}$ to induce oxidative stress, we decided to investigate whether this condition also affects the activity of this important antioxidant enzyme. For this purpose the activity of isolated TrxR1 from Rattus Norvegicus was 
measured in the presence of the various test conditions. Quercetin concentrationdependently decreased TrxR1 activity $(10 \mu \mathrm{M}: 22.4 \pm 3.3 \% ; 50 \mu \mathrm{M}: 45.1 \pm 3.2 \%$; Figure $4 \mathrm{~A}$ ), while $25 \mathrm{U}$ tyrosinase only slightly reduced $(9.7 \pm 3.2 \%$ ) the activity of TrxR1 (Figure $4 A)$. Quercetin quinone generated in the presence of quercetin and tyrosinase inhibited TrxR1 activity (10 $\mu \mathrm{M}$ : $91.4 \pm 3.5 \% ; 50 \mu \mathrm{M}$ : $100.9 \pm 2.8 \%)$ almost completely, which corresponded to the inhibition achieved by the positive control (60), $150 \mathrm{nM}$ auranofin (78.8 $\pm 2.7 \%$; Figure 4A). One mM GSH did not affect the TrxR1 inhibition induced by auranofin, but almost completely protected against the inhibition by quercetin quinone (Figure 4B).

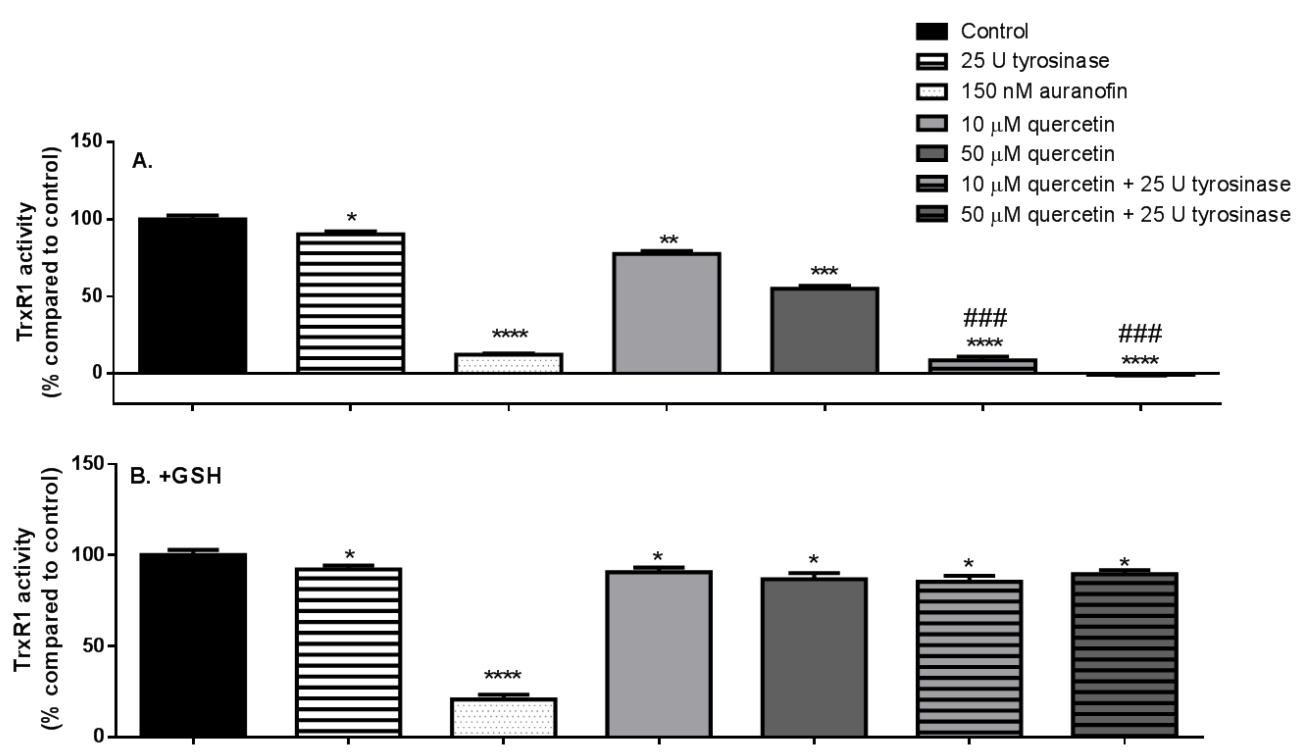

Figure 4: TrxR1 activity in percentage compared to control activity of TrxR1 isolated from Rattus norvegicus, after incubation with $25 \mathrm{U}$ tyrosinase, $150 \mathrm{nM}$ auranofin, $10 \mu \mathrm{M}$ quercetin, $50 \mu \mathrm{M}$ quercetin, $10 \mu \mathrm{M}$ quercetin and $25 \mathrm{U}$ tyrosinase, $50 \mu \mathrm{M}$ quercetin and $25 \mathrm{U}$ tyrosinase for $15 \mathrm{~min}$. in absence of (A) or presence of $1 \mathrm{mM}$ GSH (B). Twenty-five $\mathrm{U}$ tyrosinase was used to induce oxidative stress, while quercetin quinone was produced by adding $25 \mathrm{U}$ tyrosinase to quercetin. $\mathrm{N}=3$ and data are presented as mean \pm SEM. * $\mathrm{P}<$ $0.05 ; * * \mathrm{P}<0.01 ; * * * \mathrm{P}<0.001$ compared to control. \#\#\#P<0.001 compared to the condition of $25 \mathrm{U}$ tyrosinase to induce oxidative stress. 


\subsection{Quercetin quinone did not inhibit TrxR1 activity in A375 cells independent of the presence of GSH}

TrxR1 activity was examined in A375 cells exposed to the various test conditions. Absolutely, the TrxR1 activity did not differ significantly between non-treated and BSOtreated cells (data not shown). Independent of the intracellular GSH levels, $140 \mu \mathrm{M}$ quercetin, $200 \mu \mathrm{M} \mathrm{H}_{2} \mathrm{O}_{2}$ and $140 \mu \mathrm{M}$ quercetin combined with $200 \mu \mathrm{M} \mathrm{H}_{2} \mathrm{O}_{2}$ for 45 min did not affect TrxR1 activity (Figure 5). However, auranofin significantly diminished TrxR1 activity, both in the presence (105.0 $\pm 12.3 \%)$ and absence of GSH (87.7 $\pm 15.3 \%$ ) (Figure 5). The TrxR1 activity measured after exposing BSO-treated A375 cells to $1 \mu \mathrm{M}$ auranofin did not differ significantly from zero (= no activity).
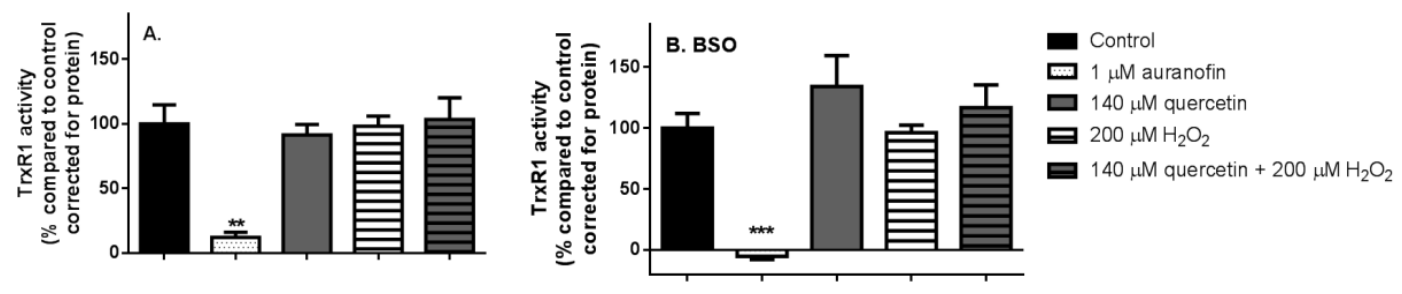

Figure 5: TrxR1 activity corrected for protein and shown in percentage compared to control activity of TrxR1 in A375 cells incubated with the mentioned drugs for $\mathbf{4 5}$ min. The effects on TrxR1 activity due to $1 \mu \mathrm{M}$ auranofin, $140 \mu \mathrm{M}$ quercetin, $200 \mu \mathrm{M} \mathrm{H}_{2} \mathrm{O}_{2}$ to induce oxidative stress or quercetin quinone generated by addition of $200 \mu \mathrm{M} \mathrm{H}_{2} \mathrm{O}_{2}$ to $140 \mu \mathrm{M}$ quercetin were shown in non-pretreated A375 cells or B) in A375 cells depleted with GSH (= BSO-treated). N=3 and data are presented as mean \pm SEM. *P $<0.05 ; * * \mathrm{P}<$ $0.01 ; * * * \mathrm{P}<0.001$ compared to control.

\subsection{Quercetin quinone induced apoptosis, while oxidative stress induced necrosis in BSO-treated A375 cells}

In order to assess the cytotoxic effects of the different test conditions on A375 cells, apoptotic and necrotic cells were quantified after annexin $V$ and $7 A A D$ labeling of all (i.e. CD44+) cells by flow cytometry.

Twohundred $\mu \mathrm{M} \mathrm{H}_{2} \mathrm{O}_{2}$ added to induce oxidative stress hardly affected the number of apoptotic cells (Figure $6 \mathrm{~A}$ ) but resulted in a significant rise in the number of 202 
7AAD-positive cells (12.8 $\pm 5.6 \%$; Figure $6 \mathrm{~B})$ compared to unexposed cells. The significantly increased LDH leakage ( $11.1 \pm 8.2 \%$ compared to unexposed cells; Figure $6 \mathrm{C}$ ) supported the finding that $\mathrm{H}_{2} \mathrm{O}_{2}$ induced necrosis rather than apoptosis in BSO-treated A375 cells under these test conditions. In contrast, quercetin quinone generated by the simultaneous addition of $140 \mu \mathrm{M}$ quercetin and $200 \mu \mathrm{M} \mathrm{H}_{2} \mathrm{O}_{2}$ increased annexin $V$ labeling after $24 \mathrm{~h}\left(17.7 \pm 4.0 \%\right.$ ) compared to $\mathrm{H}_{2} \mathrm{O}_{2}$ alone (Figure $6 \mathrm{~A}$ ), indicating that quercetin quinone promoted apoptosis.
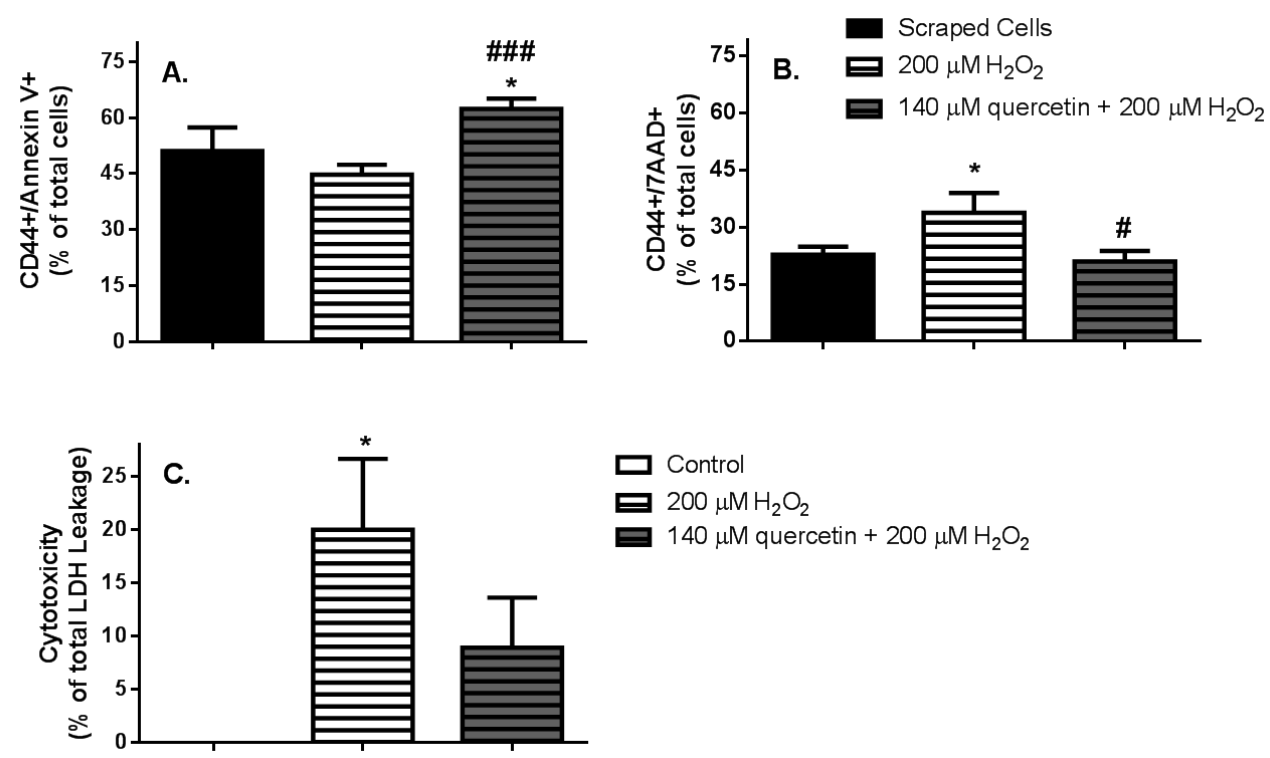

Figure 6: FACS analysis to determine apoptosis (annexin V+, A) and necrosis (7AAD+, B) in BSO-treated A375 cells (CD44+). As a (positive) control for both apoptotic and necrotic A375 cells scraped cells were used. FACS analysis was done $24 \mathrm{~h}$ after exposure. C) Cytotoxicity in BSO-treated A375 cells shown as percentage of total LDH leakage induced by $3 \%(v / v)$ Triton X-100. Twohundred $\mu \mathrm{M} \mathrm{H}_{2} \mathrm{O}_{2}$ was used to induce oxidative stress. Quercetin quinone was formed by adding $200 \mu \mathrm{M} \mathrm{H} \mathrm{H}_{2}$ in combination with $140 \mu \mathrm{M}$ quercetin to the cells. $\mathrm{N}=3$ and data are presented as mean $\pm \mathrm{SEM}$. ${ }^{*} \mathrm{P}<0.05 ; * \mathrm{*}<0.01$; $* * * \mathrm{P}<0.001$ compared to control, \#P<0.05; \#\#\#P<0.001 compared to the condition of $200 \mu \mathrm{M} \mathrm{H}_{2} \mathrm{O}_{2}$. 


\subsection{Quercetin quinone did not affect the inhibition of glycolytic activity of GAPDH induced by $\mathrm{H}_{2} \mathrm{O}_{2}$}

Next, the mechanism underlying the quercetin quinone-induced apoptosis was further explored. It has been previously suggested that GAPDH levels were increased in tumor cells compared to control cells. As a result of nitrosative stress GAPDH could translocate to the nucleus inducing apoptosis by increasing the expression of apoptosisrelated genes like TP53 and pro-apoptotic CASP8 or decreasing the expression of antiapoptotic BCL2 (61-64). Therefore, the effect of the test conditions on GAPDH activity, nuclear translocation and expression of apoptotic related genes was determined in BSOtreated A375 cells. First the effect of the various test conditions on the activity of isolated GAPDH was determined (Supplementary Figure 3). Similarly, quercetin did not affect

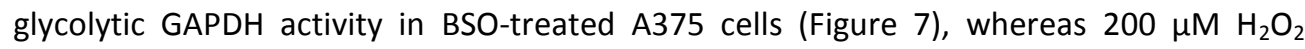
significantly inhibited the activity of GAPDH by $72.6 \pm 18.7 \%$ compared to control after 24 $\mathrm{h}$ (Figure 7). Quercetin quinone, formed in the presence of $140 \mu \mathrm{M}$ quercetin and $200 \mu \mathrm{M}$ $\mathrm{H}_{2} \mathrm{O}_{2}$ had no effect on the $\mathrm{H}_{2} \mathrm{O}_{2}$-induced decline in GAPDH activity (Figure 7).

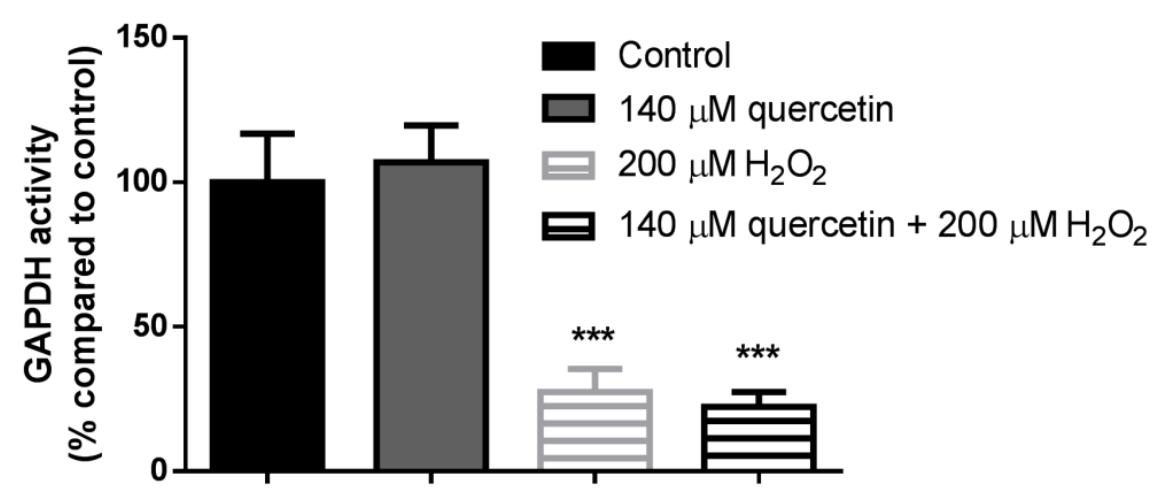

Figure 7: GAPDH activity corrected for protein and shown in percentage compared to control activity of GAPDH in BSO-treated A375 cells exposed to the indicated conditions for $45 \mathrm{~min}$. Twohundred $\mu \mathrm{M} \mathrm{H}_{2} \mathrm{O}_{2}$ was used to induce oxidative stress. Quercetin quinone was generated by adding the combination of $200 \mu \mathrm{M} \mathrm{H} \mathrm{H}_{2} \mathrm{O}_{2}$ with $140 \mu \mathrm{M}$ quercetin. $\mathrm{N}=3$ and data are presented as mean \pm SEM. ${ }^{*} \mathrm{P}<0.05 ;{ }^{*} \mathrm{P}<0.01 ;{ }^{* * *} \mathrm{P}<$ 0.001 compared to control. 


\subsection{Quercetin quinone increased GAPDH nuclear translocation}

Next, the effects of all test conditions were assessed on the nuclear translocation of GAPDH in BSO-treated A375 cells after $24 \mathrm{~h}$. Quercetin had no effect on GAPDH nuclear translocation in $\mathrm{A} 375$ cells compared to control cells (Figure 8). While $200 \mu \mathrm{M} \mathrm{H} \mathrm{O}_{2}$ to induce oxidative stress elevated the nuclear presence of GAPDH by $60.3 \pm 12.7 \%$, quercetin quinone generated by the combined exposure to $140 \mu \mathrm{M}$ quercetin and $200 \mu \mathrm{M}$ $\mathrm{H}_{2} \mathrm{O}_{2}$ further raised GAPDH presence in the nucleus by $112.1 \pm 86.2 \%$ (Figure 8).

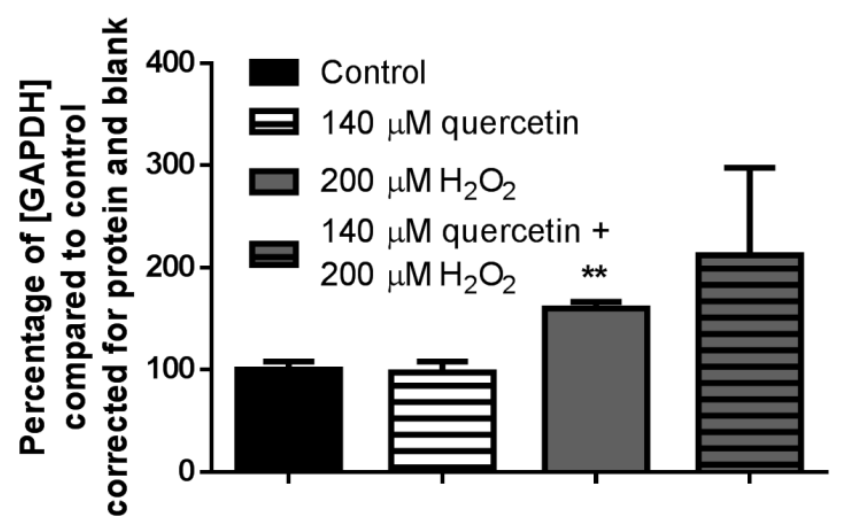

Figure 8: Nuclear GAPDH shown as a percentage compared to the concentration of GAPDH in the nucleus in control cells. All samples were corrected for protein. Twohundred $\mu \mathrm{M} \mathrm{H}_{2} \mathrm{O}_{2}$ was used to induce oxidative stress and quercetin quinone was generated by adding the combination of $140 \mu \mathrm{M}$ quercetin and $200 \mu \mathrm{M} \mathrm{H}_{2} \mathrm{O}_{2}$ to the BSO-

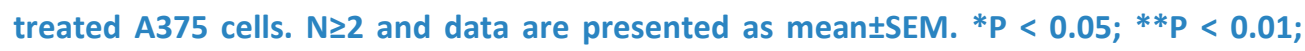
$* * * \mathrm{P}<0.001$ compared to control.

\subsection{Quercetin quinone enhanced apoptotic gene expression in melanoma cells (compared to unexposed control cells)}

Finally, we examined in how far the observed effects of the test conditions on GAPDH translocation were associated with changes in the expression of apoptotic genes in BSO-treated A375 cells after $24 \mathrm{~h}$. As it can be seen in Figure 9A, $200 \mu \mathrm{M} \mathrm{H}_{2} \mathrm{O}_{2}$ diminished TP53 expression by $29.3 \pm 9.7 \%$ compared to control cells. Quercetin quinone formed by the simultaneous exposure to $140 \mu \mathrm{M}$ quercetin and $200 \mu \mathrm{M} \mathrm{H} \mathrm{H}_{2} \mathrm{O}_{2}$ attenuated this decrease in TP53 expression induced by $\mathrm{H}_{2} \mathrm{O}_{2}$ (Figure 9A). Moreover, both, $\mathrm{H}_{2} \mathrm{O}_{2}(2.2 \pm 1.6$ 
fold) and quercetin quinone ( $1.7 \pm 1.2$ fold) increased the ratio between the expression of the pro-apoptotic CASP8 and the anti-apoptotic BCL2 gene. This finding indicates that under these test conditions a higher expression of pro-apoptotic genes than antiapoptotic genes could be seen compared to control cells (Figure 9B).
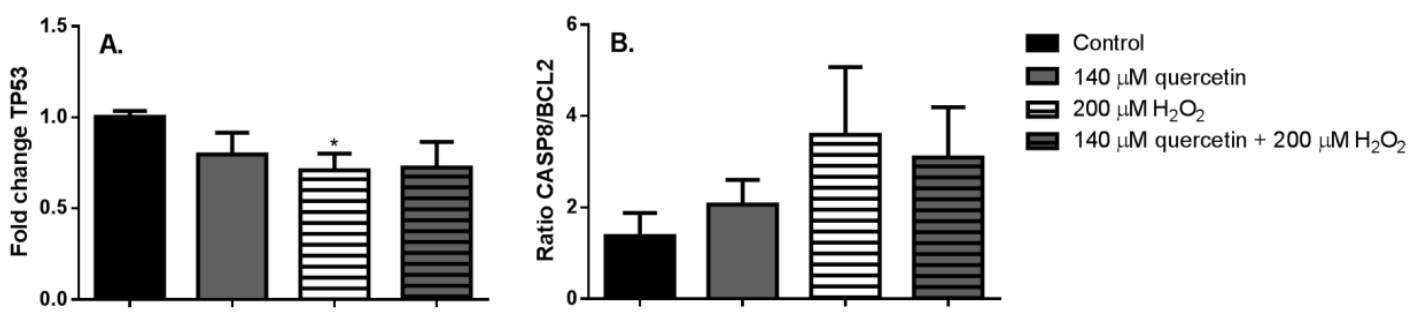

Figure 9: Expression of apoptosis-related genes in A375 cells $24 \mathrm{~h}$ after exposure to 140 $\mu \mathrm{M}$ quercetin, $200 \mu \mathrm{M} \mathrm{H} \mathrm{O}_{2}$ or quercetin quinone. Twohundred $\mu \mathrm{M} \mathrm{H}_{2} \mathrm{O}_{2}$ was used to induce oxidative stress and quercetin quinone was made by adding the combination of $140 \mu \mathrm{M}$ of quercetin and $200 \mu \mathrm{M}$ of $\mathrm{H}_{2} \mathrm{O}_{2}$ to BSO-treated A375 cells. A) TP53 mRNA levels were shown. $B$ ) The ratio of expression of the pro-apoptotic gene CASP8 relative to the anti-apoptotic BCL2 was shown. $\mathrm{N}=3$ and data are presented as mean \pm SEM. ${ }^{*} \mathrm{P}<$ $0.05 ; * * \mathrm{P}<0.01 ; * * * \mathrm{P}<0.001$ compared to control.

\section{Discussion}

It has been reported that quercetin intake is negatively associated with the incidence of oxidative stress-induced diseases like cancer $(65,66)$, but the molecular mechanism is still enigmatic. It is generally accepted that quercetin acts as an antioxidant by scavenging ROS (12). During this scavenging quercetin is oxidized to quercetin quinone that can induce adaptation by activating Nrf2-mediated transcription of endogenous antioxidant genes. Since this would protect tumor cells (67-69), which is undesirable in a therapeutic context, it prompted us to investigate the effect of quercetin quinone in BSOtreated A375 melanoma tumor cells.

First, in non-tumor cells, specifically HEK293 $\left(\mathrm{pTRAF}^{\mathrm{Nrf2} / \mathrm{HIF} / \mathrm{NF}-\mathrm{kB}}\right.$ ) reporter cells, we investigated the effect of quercetin quinone on the activity of transcription factors Nrf2, NF-KB and HIF. First of all, a decreased Nrf2 activity diminishes cellular protection against oxidative stress because of reduced upregulation of the endogenous antioxidant response. Less endogenous antioxidants lead to less protection against oxidative stress and increases the risk of a non-tumor cell to develop in a tumor cell, because of the increased 206 
risk for oxidative damage $(18,22,70-72)$. Second, an enhanced HIF activity has been associated with more angiogenesis, which is associated with tumor metastasis (73). HIF and Nrf2 have also been shown to be upregulated in cancer stem cells, a specific type of cancer cells that are highly resistant and metastatic, but do not have a proliferation rate as high as cancer cells without stem cell phenotype (74). Finally, increased NF-kB activity is associated with more inflammation and proliferation (75) In non-tumor cells, specifically HEK293 (pTRAF ${ }^{\mathrm{Nrf2} / \mathrm{HIF} / \mathrm{NF}-\mathrm{kB}}$ ) reporter cells, we showed that quercetin quinone decreases the activity of the transcription factors HIF, NF-KB, and increases the activity of Nrf2. In this way, quercetin quinone could protect non-tumor HEK293 $\left(\right.$ pTRAF $^{\mathrm{Nrf2} / \mathrm{HIF} / \mathrm{NF}-\mathrm{kB}}$ ) reporter cells from developing in tumor cells, because quercetin quinone alters the markers Nrf2, HIF, NF-KB which have been associated with a poor prognosis.

In contrast in a later stadium, cancer cells have upregulated Nrf2 activity, which is associated with a poor prognosis, because it reflects the redox imbalance and protects the tumor cell from oxidative damage (23). The increased activity of Nrf2 in A375 cells compared to HEK293 cells has been confirmed previously $(51,74)$. The combination of high endogenous antioxidant levels to cope with higher endogenous metabolism and addition of BSO in A375 cells has previously been shown not to affect toxicity or cleavage of caspases (49, 76-78). However, the level of oxidative stress appears to be increased (78, 79). Remarkably, in BSO-treated $A 375$ cells we found that $\mathrm{H}_{2} \mathrm{O}_{2}$-mediated oxidative stress further enhances Nrf2-dependent gene expression as seen by a probably compensatory increased expression of HMOX1 and TXNRD1, whereas the combination of quercetin and $200 \mu \mathrm{M} \mathrm{H}_{2} \mathrm{O}_{2}$ appears to suppress Nrf2-mediated gene expression of HMOX1 and TXNRD1. Although the effect of other transcription factors e.g. activator protein-1 (AP-1) regulating for example HMOX1 should not be excluded (80). A high level of oxidative stress and overexpression of specific proteins i.e. Nrf2, TrxR1 and GAPDH in melanoma cells could explain these results, because more oxidative stress favors the formation of quercetin quinone that can react with more thiol-containing proteins $(19,81-84)$. In contrast, GCLC gene expression was not affected by quercetin quinone. This could be explained by the use of BSO that results in a low level of GSH after $24 \mathrm{~h}(85,86)$, and probably increases $\mathrm{GCLC}$ gene expression during the following $24 \mathrm{~h}$ exposure to quercetin and oxidative stress.

A375 melanoma cells were selected due to the constitutive presence of tyrosinase in these cells enabling the intracellular in situ production of quercetin quinone. Quercetin alone had no effect on endogenous antioxidant gene expression in contrast to the combination of quercetin with either tyrosinase or $\mathrm{H}_{2} \mathrm{O}_{2}$. This suggests that the endogenous tyrosinase in the cell is not involved in the in situ formation of quercetin quinone. This can be explained by the exclusive localization of tyrosinase in specific endosomes, called melanosomes, whereas quercetin is mainly present in the cytoplasm of 
the cell, indicating that cellular tyrosinase does not interact with quercetin to generate the quinone $(8,9,87)$.

Since we observed that quercetin reduced Nrf2-regulated expression of the TXNRD1 gene in the pro-oxidant, GSH depleted cancer cells, we decided to investigate whether these conditions also lead to an inhibition of TrxR1 activity. We confirmed that quercetin inhibits the activity of isolated TrxR1 (88). In addition, we have shown that tyrosinase decreases TrxR1 activity. Interestingly, in the presence of tyrosinase to induce oxidative stress, quercetin inhibited TrxR1 reductive activity more potently than quercetin or tyrosinase alone. Inhibition of TrxR1 in cancer cells would lead to less endogenous antioxidant protection and would enhance the susceptibility of the cancer cells for oxidative stress-induced damage. Although in our BSO-treated A375 cells we could not measure an inhibitory effect on TrxR1 by quercetin in the presence of tyrosinase. The inhibition by auranofin was confirmed. Remarkably auranofin inhibited TrxR1 independent of the GSH concentration. This could be due to the fact that auranofin has been reported to irreversibly bind to the selenocysteine of the enzyme $(89,90)$. The experiments with isolated TrxR1 suggested that inhibition of the enzyme by quercetin quinone was reversible by adding GSH. Due to such reversible nature of the inhibition we might have missed the inhibition of TrxR1 in the cells, e.g. because we either determined the TrxR1 inhibition too late after exposure of the cells to quercetin quinone induced by addition of quercetin and tyrosinase (21) or because the inhibition was lost during e.g. cell lysis.

Remarkably, next to the reduction of the antioxidant defense in GSH depleted A375 cells, we found that quercetin quinone also induces apoptosis in these tumor cells, shown by phosphatidylserine binding of annexin $V$ (91) (Figure 10B). In contrast, 7AAD binding of released DNA and LDH leakage (92) suggested that $\mathrm{H}_{2} \mathrm{O}_{2}$ induces necrosis rather than apoptosis.

Recent evidence suggests that the moon-lighting protein GAPDH could be involved in induction of apoptosis (93). We confirmed that $\mathrm{H}_{2} \mathrm{O}_{2}$ induces inhibition of the glycolytic GAPDH activity (55, 94-100) (Figure 10A), and that quercetin in the presence of $\mathrm{H}_{2} \mathrm{O}_{2}$ does not further reduce the metabolic activity of GAPDH (Figure 10B). The present study confirmed that $\mathrm{H}_{2} \mathrm{O}_{2}$ increases GAPDH nuclear translocation $(101,102)$ which was almost doubled when cells were exposed additionally to quercetin. This indicates that quercetin quinone promotes the function of GAPDH as a transcription factor (Figure 10B). Previously, nuclear presence of GAPDH has been associated with increased GAPDH mediated expression of $\operatorname{TP} 53(61,63,64,103-105)$ that subsequently influences the expression of other apoptotic genes (106-108). In this study, quercetin quinone increased pro-apoptotic CASP8 expression and diminished anti-apoptotic BCL2 expression (Figure 
10B), although these effects were statistically not significant. This could be attributed to several reasons. Firstly, we measured mRNA levels of these genes, and mRNA and protein levels are not always consistently expressed $(109,110)$. Secondly, caspases are present as pro-caspases, which are cleaved when apoptosis is induced. Thus, induction of apoptosis does not always require further gene expression of pro-caspases $(109,110)$. Thirdly, we measured the expression of apoptotic genes at $24 \mathrm{~h}$, whereas some genes have their maximum expression level at $72 \mathrm{~h}$ (111).

Finally, we found that quercetin in the pro-oxidant environment of BSO-treated A375 cells prevents a further decline of TP53 expression by $\mathrm{H}_{2} \mathrm{O}_{2}$ (Figure 10B). Although, this finding is not significant, it could be explained by the characteristic of most tumor cells to have TP53 mutations (112), thereby contributing to tumor development. Additionally, next to expression of TP53, GAPDH can also induce apoptosis via other pathways including changes in mitochondrial membrane potential leading to apoptosis or caspaseindependent cell death $(11,63,113,114)$.

Remarkably, we could observe that the addition of $\mathrm{H}_{2} \mathrm{O}_{2}$ to induce oxidative stress increases pro-apoptotic compared to anti-apoptotic gene expression, whereas the overall cytotoxic effect was necrotic and not apoptotic. Therefore, we hypothesize that $\mathrm{H}_{2} \mathrm{O}_{2}$ may start to initiate apoptosis, but this process is overruled by the oxidative damage of e.g. cell membranes, DNA and proteins which eventually leads to necrosis (115). The disadvantage of a necrotic cell death instead of apoptosis is that the cell content is released in the surrounding tissue leading to inflammation and further contributing to tumor cell proliferation $(116,117)$. In this respect the quercetin-mediated shift from $\mathrm{H}_{2} \mathrm{O}_{2}$-induced adaptation to an apoptotic cell death of cancer cells could be of therapeutic interest and adds to recent studies which suggest to use quercetin as an additional therapy against melanoma $(35,97-100)$. Moreover, our findings are in line with in vivo studies that revealed that flavonoids wth a comparable structure as quercetin like monoHER, apigenin and 2',5'-dihydroxychalcone enhance the anti-tumor effect of the chemotherapeutics doxorubicin or cisplatin by increasing apoptosis (31-34, 36-40). 
A. BSO-treated A375 tumor cells

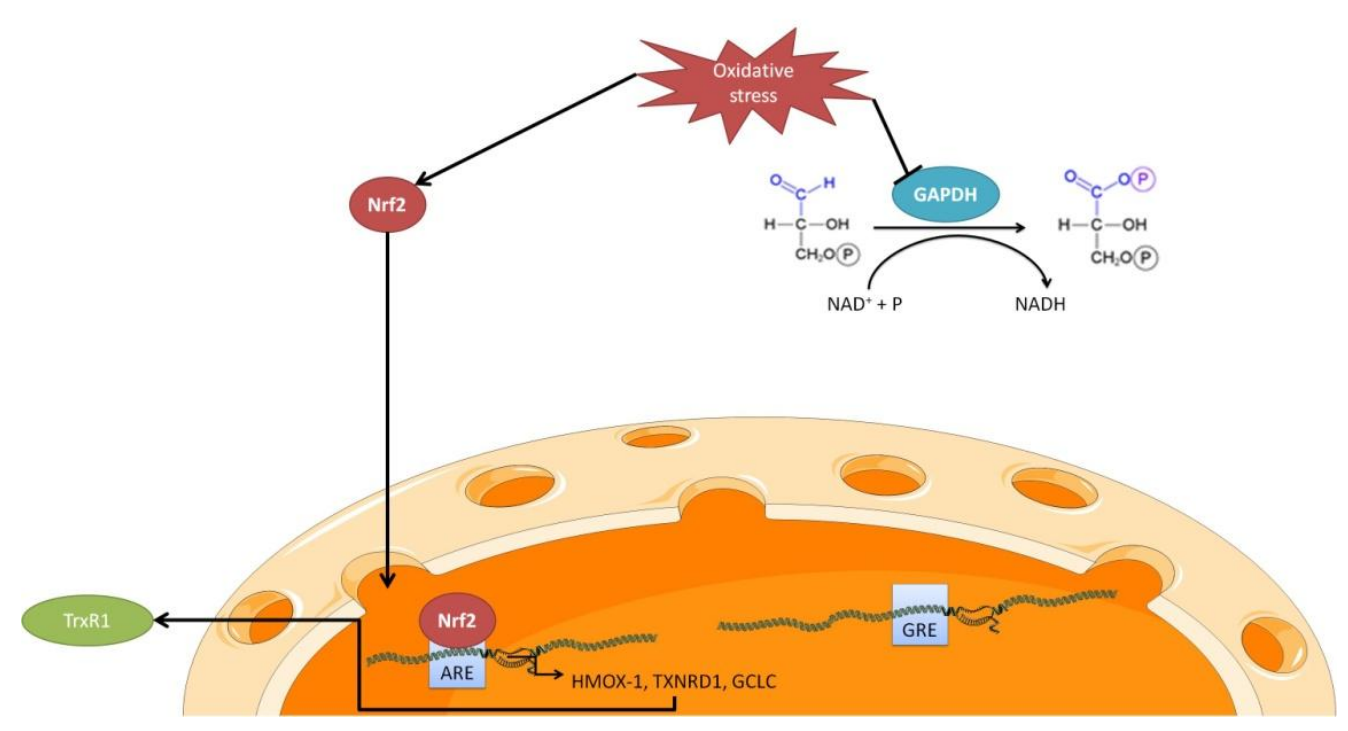

B. BSO-treated A375 tumor cells

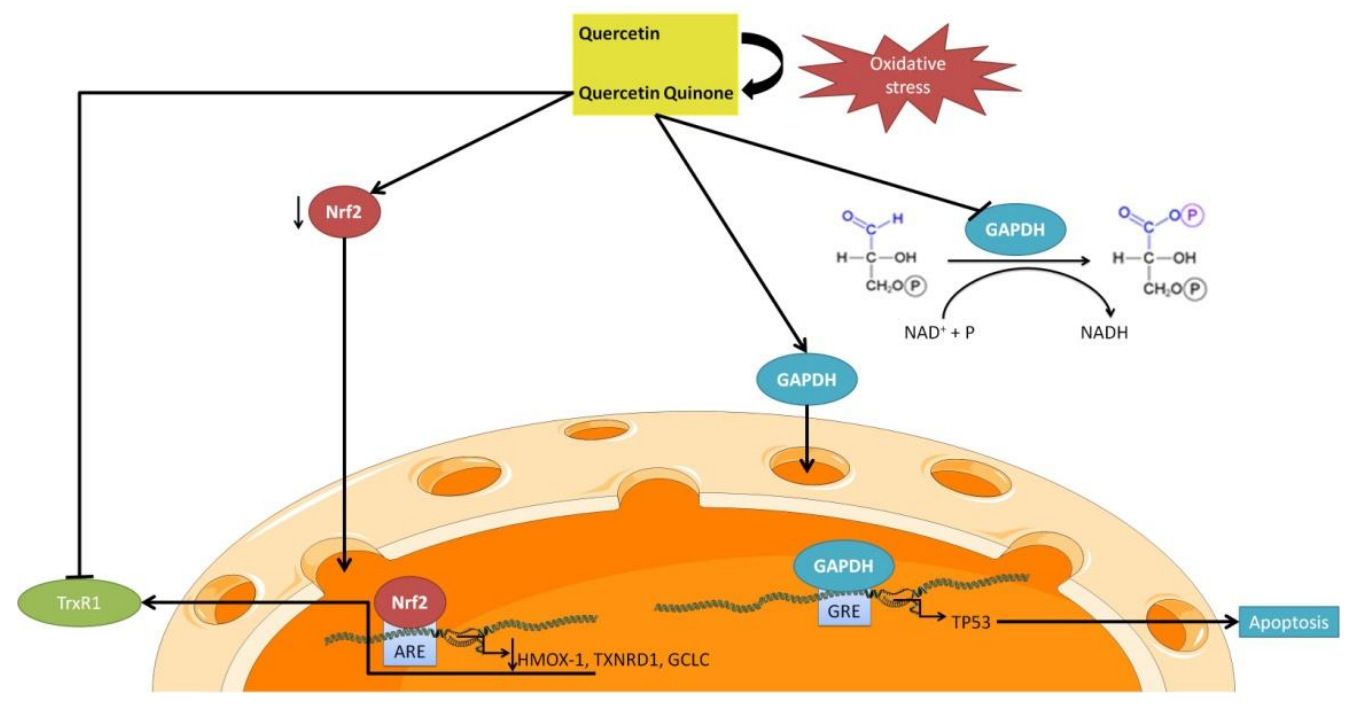

Figure 10: A) In BSO-treated A375 tumor cells, $\mathrm{H}_{2} \mathrm{O}_{2}$ induces oxidative stress, enhances Nrf2-mediated gene expression of endogenous antioxidant enzymes HMOX-1, TXNRD1 and GCLC and inhibits GAPDH glycolytic activity. B) Addition of either tyrosinase or $\mathrm{H}_{2} \mathrm{O}_{2}$ to induce oxidative stress results in the conversion of quercetin to quercetin quinone that decreases protection by inhibiting TrxR1 activity and decreasing the Nrf2-mediated endogenous antioxidant response. In addition, next to inhibiting GAPDH glycolytic activity, quercetin quinone also enhances apoptosis by inducing GAPDH-mediated 210 
expression of apoptotic genes e.g. TP53. GAPDH = glyceraldehyde-3-phosphate dehydrogenase; $\mathrm{NADH}=$ reduced nicotinamide adenine dinucleotide; $\operatorname{TrxR1}=$ thioredoxin reductase $1 ;$ TXNRD1 = gene for thioredoxin reductase $1 ;$ Nrf2 = nuclear factor (erythroid-derived 2)-like 2; ARE= antioxidant response element; HMOX-1 = heme oxygenase 1; GCLC = glutamate cysteine ligase, catalytic subunit; GRE= GAPDH response element.

\section{Conclusion}

Quercetin quinone might modulate the cellular response differently in BSOtreated A375 tumor cells compared to non-tumor HEK293 (pTRAF ${ }^{\mathrm{Nr} 2 / \mathrm{HIF} / \mathrm{NF}-\mathrm{kB} \text { ) }}$ reporter cells. In non-tumor HEK293 (pTRAF ${ }^{\mathrm{Nr} 2 / \mathrm{HIF} / \mathrm{NF}-\mathrm{kB} \text { ) }}$ reporter cells, quercetin shifts the response from cell death to adaptation. In BSO-treated A375 tumor cells, where the adaptive response is already switched on, quercetin shifts the adaptive response to cell death.

\section{Supplement}

\subsection{Supplementary Material \& Methods}

\subsubsection{Activity of isolated GAPDH}

GAPDH activity was measured as previously described (55) with some minor modifications. First, $0,25 \mathrm{U} / \mathrm{mL}$ GAPDH from rabbit muscle was incubated with either 250 $\mathrm{U} / \mathrm{mL}$ tyrosinase, $10 \mu \mathrm{M}$ quercetin, $50 \mu \mathrm{M}$ quercetin, or the combinations of either 250 $\mathrm{U} / \mathrm{mL}$ tyrosinase and $10 \mu \mathrm{M}$ quercetin or $250 \mathrm{U} / \mathrm{mL}$ tyrosinase and $50 \mu \mathrm{M}$ quercetin at $37^{\circ} \mathrm{C}$ for $5 \mathrm{~min}$. Thereafter, a mixture of $1 \mathrm{mM} \mathrm{NAD}^{+}$with $1.5 \mathrm{mM} \mathrm{G3P}$ was added and the absorbance was determined for $1 \mathrm{~min}$ at $37^{\circ} \mathrm{C}$ and $\lambda=340 \mathrm{~nm}$ using the Spectramax microplate reader (Molecular Devices). The experiment was repeated in the presence of $10 \mathrm{mM}$ GSH. 


\subsection{Supplementary Results}

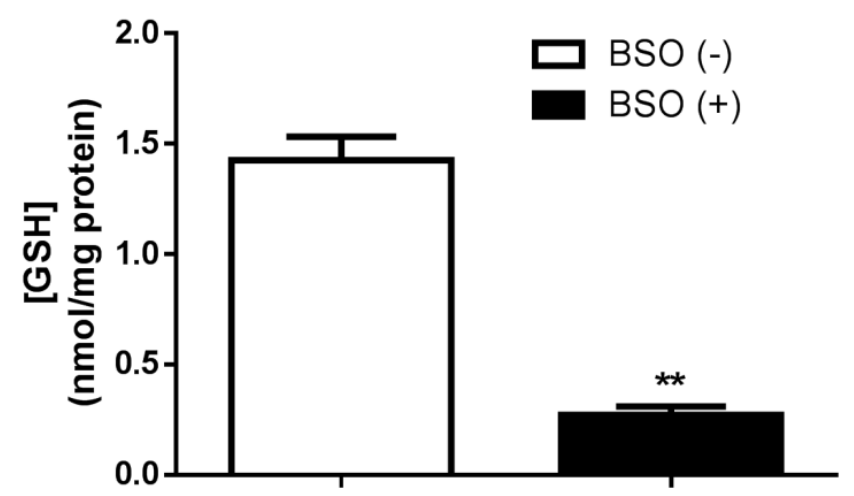

Supplementary Figure 1: Intracellular GSH concentration in A375 cells exposed to 250 $\mu \mathrm{M}$ buthionine sulfoximine (BSO) for $24 \mathrm{~h}$ or not. $\mathrm{N}=4$ and data are presented as mean \pm SEM. $* \mathrm{P}<0.05 ; * * \mathrm{P}<0.01 ; * * * \mathrm{P}<0.001$ compared to control.

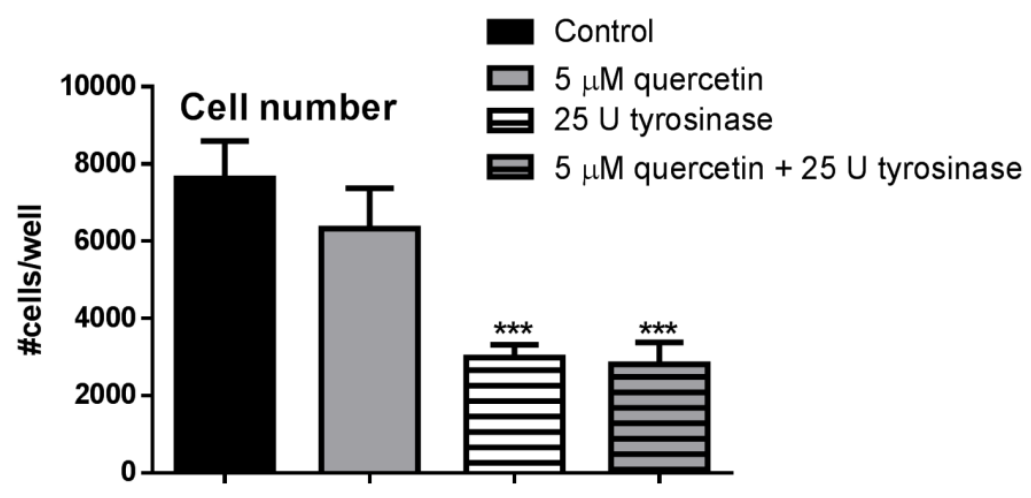

Supplementary Figure 2: Number of DAPI stained nuclei in a stable HEK(pTRAF ${ }^{\mathrm{Nr} 2 / \mathrm{HIF} / \mathrm{NF}-}$ ${ }^{\mathrm{KB}}$ ) reporter cell line exposed to indicated drugs for $24 \mathrm{~h}$. Twenty-five $U$ tyrosinase is used to induce oxidative stress. Quercetin quinone is generated by adding the combination of $25 \mathrm{U}$ tyrosinase and $5 \mu \mathrm{M}$ quercetin. ${ }^{*} \mathrm{P}<0.05 ; * \mathrm{P}<0.01 ; * * * \mathrm{P}<0.001$ compared to control, \#P<0.05 compared to the condition of $25 \mathrm{U}$ tyrosinase. 


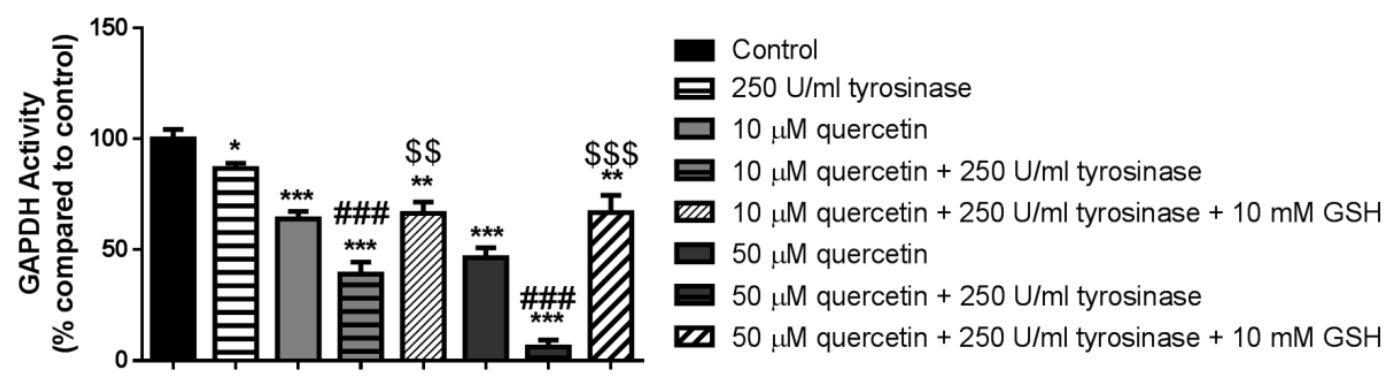

Supplementary Figure 3: GAPDH activity in percentage compared to control activity of isolated GAPDH from rabbit muscle, after incubation with $250 \mathrm{U} / \mathrm{ml}$ tyrosinase to induce oxidative stress, $10 \mu \mathrm{M}$ quercetin, quercetin quinone generated by adding $250 \mathrm{U} / \mathrm{ml}$ tyrosinase to $10 \mu \mathrm{M}$ quercetin and quercetin quinone in combination with $10 \mathrm{mM}$ GSH for $5 \mathrm{~min}$. A similar incubation was performed for $50 \mu \mathrm{M}$ quercetin. $\mathrm{N}=4$ and data are presented as mean \pm SEM. $* \mathrm{P}<0.05$; $* * \mathrm{P}<0.01 ; * * * \mathrm{P}<0.001$ compared to control. $\# \# \# \mathbf{P}<0.001$ compared to the condition of $250 \mathrm{U} / \mathrm{ml}$ tyrosinase. $\$ \$ P<0.01 ; \$ \$ \$<0.001$ compared to the same condition but without exposure to $10 \mathrm{mM} \mathrm{GSH}$. 


\section{References}

1. Manach C, Scalbert A, Morand C, Remesy C, Jimenez L. Polyphenols: food sources and bioavailability. The American journal of clinical nutrition. 2004 May;79(5):727-47.

2. Nishimuro $H$, Ohnishi $H$, Sato $M$, Ohnishi-Kameyama $M$, Matsunaga I, Naito $S$, et al. Estimated daily intake and seasonal food sources of quercetin in Japan. Nutrients. 2015 Apr 02;7(4):2345-58.

3. Egert S, Wolffram S, Bosy-Westphal A, Boesch-Saadatmandi C, Wagner AE, Frank J, et al. Daily quercetin supplementation dose-dependently increases plasma quercetin concentrations in healthy humans. The Journal of nutrition. 2008 Sep;138(9):1615-21.

4. de Boer VC, Dihal AA, van der Woude H, Arts IC, Wolffram S, Alink GM, et al. Tissue distribution of quercetin in rats and pigs. The Journal of nutrition. 2005 Jul;135(7):1718-25.

5. Carbonaro M, Grant G. Absorption of quercetin and rutin in rat small intestine. Annals of nutrition \& metabolism. 2005 May-Jun;49(3):178-82.

6. Yeh SL, Lin YC, Lin YL, Li CC, Chuang CH. Comparing the metabolism of quercetin in rats, mice and gerbils. European journal of nutrition. 2016 Feb;55(1):413-22.

7. Jacobs $H$, Koek GH, Peters R, Moalin M, Tack J, van der Vijgh WJ, et al. Differences in pharmacological activities of the antioxidant flavonoid monoHER in humans and mice are caused by variations in its metabolic profile. Clin Pharmacol Ther. 2011 Dec;90(6):852-9.

8. Nait Chabane M, Al Ahmad A, Peluso J, Muller CD, Ubeaud G. Quercetin and naringenin transport across human intestinal Caco-2 cells. The Journal of pharmacy and pharmacology. 2009 Nov;61(11):1473-83.

9. Walle T, Vincent TS, Walle UK. Evidence of covalent binding of the dietary flavonoid quercetin to DNA and protein in human intestinal and hepatic cells. Biochemical pharmacology. 2003 May 15;65(10):1603-10.

10. Boots AW, Drent M, de Boer VC, Bast A, Haenen GR. Quercetin reduces markers of oxidative stress and inflammation in sarcoidosis. Clinical nutrition. 2011 Aug;30(4):506-12.

11. Guiney SJ, Adlard PA, Bush Al, Finkelstein DI, Ayton S. Ferroptosis and cell death mechanisms in Parkinson's disease. Neurochem Int. 2017 Jan 09. 
12. Lemmens KJ, van de Wier B, Vaes N, Ghosh M, van Zandvoort MA, van der Vijgh WJ, et al. The flavonoid 7-mono-O-(beta-hydroxyethyl)-rutoside is able to protect endothelial cells by a direct antioxidant effect. Toxicology in vitro : an international journal published in association with BIBRA. 2014 Jun;28(4):538-43.

13. Manach C, Williamson G, Morand C, Scalbert A, Remesy C. Bioavailability and bioefficacy of polyphenols in humans. I. Review of 97 bioavailability studies. The American journal of clinical nutrition. 2005 Jan;81(1 Suppl):230S-42S.

14. Boots AW, Kubben N, Haenen GR, Bast A. Oxidized quercetin reacts with thiols rather than with ascorbate: implication for quercetin supplementation. Biochemical and biophysical research communications. 2003 Aug 29;308(3):560-5. 15. Jacobs $H$, Moalin $M$, Bast A, van der Vijgh WJ, Haenen GR. An essential difference between the flavonoids monoHER and quercetin in their interplay with the endogenous antioxidant network. PloS one. 2010 Nov 08;5(11):e13880.

16. Lemmens KJ, Vrolijk MF, Bouwman FG, van der Vijgh WJ, Bast A, Haenen $\mathrm{GR}$. The minor structural difference between the antioxidants quercetin and 4'Omethylquercetin has a major impact on their selective thiol toxicity. International journal of molecular sciences. 2014 Apr 30;15(5):7475-84.

17. Takaya K, Suzuki T, Motohashi H, Onodera K, Satomi S, Kensler TW, et al. Validation of the multiple sensor mechanism of the Keap1-Nrf2 system. Free radical biology \& medicine. 2012 Aug 15;53(4):817-27.

18. Sthijns MMJPE, Schiffers PM, Janssen GM, Lemmens KJA, Ides B, Vangrieken $\mathrm{P}$, et al. Rutin protects against $\mathrm{H}$ 2O2-triggered impaired relaxation of placental arterioles and induces Nrf2-mediated adaptation in Human Umbilical Vein Endothelial Cells exposed to oxidative stress. Biochimica et Biophysica Acta General Subjects. 2017;1861(5 Part A):1177-89.

19. Lemmens KJA, Sthijns MMJPE, van der Vijgh WJF, Bast A, Haenen GRMM. The antioxidant flavonoid monoHER provides efficient protection and induces the innate Nrf2 mediated adaptation in endothelial cells subjected to oxidative stress. Pharmanutrition. 2014;2(3):69-74.

20. Sthijns MM, Thongkam W, Albrecht C, Hellack B, Bast A, Haenen GR, et al. Silver nanoparticles induce hormesis in A549 human epithelial cells. Toxicology in vitro : an international journal published in association with BIBRA. 2017 Jan 18.

21. Sthijns MM, Weseler AR, Bast A, Haenen GR. Time in Redox Adaptation Processes: From Evolution to Hormesis. International journal of molecular sciences. 2016 Sep 29;17(10). 
22. Gorrini C, Harris IS, Mak TW. Modulation of oxidative stress as an anticancer strategy. Nature reviews Drug discovery. 2013 Dec;12(12):931-47.

23. Wondrak GT. Redox-directed cancer therapeutics: molecular mechanisms and opportunities. Antioxidants \& redox signaling. 2009 Dec;11(12):3013-69.

24. Fiaschi T, Chiarugi P. Oxidative stress, tumor microenvironment, and metabolic reprogramming: a diabolic liaison. Int J Cell Biol. 2012;2012:762825.

25. Jaramillo MC, Zhang DD. The emerging role of the Nrf2-Keap1 signaling pathway in cancer. Genes Dev. 2013 Oct 15;27(20):2179-91.

26. Menegon S, Columbano A, Giordano S. The Dual Roles of NRF2 in Cancer. Trends Mol Med. 2016 Jul;22(7):578-93.

27. Wang XJ, Sun Z, Villeneuve NF, Zhang S, Zhao F, Li Y, et al. Nrf2 enhances resistance of cancer cells to chemotherapeutic drugs, the dark side of Nrf2. Carcinogenesis. 2008 Jun;29(6):1235-43.

28. Nguyen P, Awwad RT, Smart DD, Spitz DR, Gius D. Thioredoxin reductase as a novel molecular target for cancer therapy. Cancer letters. 2006 May 18;236(2):164-74.

29. Trachootham D, Alexandre J, Huang P. Targeting cancer cells by ROSmediated mechanisms: a radical therapeutic approach? Nature reviews Drug discovery. 2009 Jul;8(7):579-91.

30. Kaminska KK, Bertrand HC, Tajima H, Stafford WC, Cheng Q, Chen W, et al. Indolin-2-one compounds targeting thioredoxin reductase as potential anticancer drug leads. Oncotarget. 2016 Jun 28;7(26):40233-51.

31. Bruynzeel AM, Niessen HW, Bronzwaer JG, van der Hoeven JJ, Berkhof J, Bast $A$, et al. The effect of monohydroxyethylrutoside on doxorubicin-induced cardiotoxicity in patients treated for metastatic cancer in a phase II study. $\mathrm{Br} \mathrm{J}$ Cancer. 2007 Oct 22;97(8):1084-9.

32. Chan LP, Chou TH, Ding HY, Chen PR, Chiang FY, Kuo PL, et al. Apigenin induces apoptosis via tumor necrosis factor receptor- and $\mathrm{Bcl}$-2-mediated pathway and enhances susceptibility of head and neck squamous cell carcinoma to 5-fluorouracil and cisplatin. Biochimica et biophysica acta. 2012 Jul;1820(7):1081-91.

33. Drozd E, Gruber B, Marczewska J, Drozd J, Anuszewska E. Intracellular glutathione level and efflux in human melanoma and cervical cancer cells differing in doxorubicin resistance. Postepy Hig Med Dosw (Online). 2016 Apr 18;70:319-28. 
34. Estrela JM, Ortega A, Mena S, Sirerol JA, Obrador E. Glutathione in metastases: From mechanisms to clinical applications. Crit Rev Clin Lab Sci. 2016 Aug;53(4):253-67.

35. Harris Z, Donovan MG, Branco GM, Limesand KH, Burd R. Quercetin as an Emerging Anti-Melanoma Agent: A Four-Focus Area Therapeutic Development Strategy. Frontiers in nutrition. 2016;3:48.

36. Jacobs H, Bast A, Peters GJ, van der Vijgh WJ, Haenen GR. The semisynthetic flavonoid monoHER sensitises human soft tissue sarcoma cells to doxorubicin-induced apoptosis via inhibition of nuclear factor-kappaB. Br J Cancer. 2011 Feb 01;104(3):437-40.

37. Kachadourian R, Leitner HM, Day BJ. Selected flavonoids potentiate the toxicity of cisplatin in human lung adenocarcinoma cells: a role for glutathione depletion. Int J Oncol. 2007 Jul;31(1):161-8.

38. Mena S, Benlloch M, Ortega A, Carretero J, Obrador E, Asensi M, et al. Bcl2 and glutathione depletion sensitizes B16 melanoma to combination therapy and eliminates metastatic disease. Clin Cancer Res. 2007 May 01;13(9):2658-66.

39. Pendyala L, Perez R, Weinstein A, Zdanowicz J, Creaven PJ. Effect of glutathione depletion on the cytotoxicity of cisplatin and iproplatin in a human melanoma cell line. Cancer Chemother Pharmacol. 1997;40(1):38-44.

40. Rocha CR, Garcia CC, Vieira DB, Quinet A, de Andrade-Lima LC, Munford V, et al. Glutathione depletion sensitizes cisplatin- and temozolomide-resistant glioma cells in vitro and in vivo. Cell Death Dis. 2015 Apr 16;6:e1727.

41. Salganik RI, Albright CD, Rodgers J, Kim J, Zeisel SH, Sivashinskiy MS, et al. Dietary antioxidant depletion: enhancement of tumor apoptosis and inhibition of brain tumor growth in transgenic mice. Carcinogenesis. 2000 May;21(5):909-14.

42. Albright CD, Salganik RI, Van Dyke T. Dietary depletion of vitamin E and vitamin A inhibits mammary tumor growth and metastasis in transgenic mice. J Nutr. 2004 May;134(5):1139-44.

43. Le Gal K, Ibrahim MX, Wiel C, Sayin VI, Akula MK, Karlsson C, et al. Antioxidants can increase melanoma metastasis in mice. Sci Transl Med. 2015 Oct 07;7(308):308re8.

44. Sayin VI, Ibrahim MX, Larsson E, Nilsson JA, Lindahl P, Bergo MO. Antioxidants accelerate lung cancer progression in mice. Sci Transl Med. 2014 Jan 29;6(221):221ra15. 
45. Awad HM, Boersma MG, Boeren S, van der Woude H, van Zanden J, van Bladeren PJ, et al. Identification of o-quinone/quinone methide metabolites of quercetin in a cellular in vitro system. FEBS Lett. 2002 Jun 05;520(1-3):30-4.

46. Tada M, Kohno M, Kasai S, Niwano Y. Generation mechanism of radical species by tyrosine-tyrosinase reaction. J Clin Biochem Nutr. 2010 Sep;47(2):162-6. 47. Liou GY, Storz P. Reactive oxygen species in cancer. Free Radic Res. 2010 May;44(5):479-96.

48. Jenkins NC, Grossman D. Role of melanin in melanocyte dysregulation of reactive oxygen species. Biomed Res Int. 2013;2013:908797.

49. Sedlak TW, Saleh M, Higginson DS, Paul BD, Juluri KR, Snyder SH. Bilirubin and glutathione have complementary antioxidant and cytoprotective roles. Proc Natl Acad Sci U S A. 2009 Mar 31;106(13):5171-6.

50. Prast-Nielsen S, Dexheimer TS, Schultz L, Stafford WC, Cheng Q, Xu J, et al. Inhibition of thioredoxin reductase 1 by porphyrins and other small molecules identified by a high-throughput screening assay. Free radical biology \& medicine. 2011 May 01;50(9):1114-23.

51. Johansson K, Cebula M, Rengby O, Dreij K, Carlstrom KE, Sigmundsson K, et al. Cross Talk in HEK293 Cells Between Nrf2, HIF, and NF-kappaB Activities upon Challenges with Redox Therapeutics Characterized with Single-Cell Resolution. Antioxidants \& redox signaling. 2015 Nov 11.

52. Sthijns MM, Randall MJ, Bast A, Haenen GR. Adaptation to acrolein through upregulating the protection by glutathione in human bronchial epithelial cells: the materialization of the hormesis concept. Biochemical and biophysical research communications. 2014 Apr 18;446(4):1029-34.

53. Livak KJ, Schmittgen TD. Analysis of relative gene expression data using real-time quantitative PCR and the 2(-Delta Delta C(T)) Method. Methods. 2001 Dec;25(4):402-8.

54. Arner ES, Holmgren A. Measurement of thioredoxin and thioredoxin reductase. Current protocols in toxicology. 2001 May;Chapter 7:Unit 74.

55. Rodriguez CE, Fukuto JM, Taguchi K, Froines J, Cho AK. The interactions of 9,10-phenanthrenequinone with glyceraldehyde-3-phosphate dehydrogenase (GAPDH), a potential site for toxic actions. Chemico-biological interactions. 2005 Jun 30;155(1-2):97-110.

56. Swalwell H, Latimer J, Haywood RM, Birch-Machin MA. Investigating the role of melanin in UVA/UVB- and hydrogen peroxide-induced cellular and 
mitochondrial ROS production and mitochondrial DNA damage in human melanoma cells. Free radical biology \& medicine. 2012 Feb 01;52(3):626-34.

57. Halliwell B, Clement MV, Long LH. Hydrogen peroxide in the human body. FEBS Lett. 2000 Dec 01;486(1):10-3.

58. Kubo I, Nihei K, Shimizu K. Oxidation products of quercetin catalyzed by mushroom tyrosinase. Bioorg Med Chem. 2004 Oct 15;12(20):5343-7.

59. van der Woude $H$, Alink GM, van Rossum BE, Walle K, van Steeg H, Walle T, et al. Formation of transient covalent protein and DNA adducts by quercetin in cells with and without oxidative enzyme activity. Chemical research in toxicology. 2005 Dec;18(12):1907-16.

60. Rigobello MP, Folda A, Baldoin MC, Scutari G, Bindoli A. Effect of auranofin on the mitochondrial generation of hydrogen peroxide. Role of thioredoxin reductase. Free Radic Res. 2005 Jul;39(7):687-95.

61. Zhang JY, Zhang F, Hong CQ, Giuliano AE, Cui XJ, Zhou GJ, et al. Critical protein GAPDH and its regulatory mechanisms in cancer cells. Cancer biology \& medicine. 2015 Mar;12(1):10-22.

62. Hara MR, Agrawal N, Kim SF, Cascio MB, Fujimuro M, Ozeki Y, et al. Snitrosylated GAPDH initiates apoptotic cell death by nuclear translocation following Siah1 binding. Nature cell biology. 2005 Jul;7(7):665-74.

63. Tristan C, Shahani N, Sedlak TW, Sawa A. The diverse functions of GAPDH: views from different subcellular compartments. Cellular signalling. 2011 Feb;23(2):317-23.

64. Zhai D, Chin K, Wang M, Liu F. Disruption of the nuclear p53-GAPDH complex protects against ischemia-induced neuronal damage. Molecular brain. 2014 Mar 27;7:20.

65. Angst E, Park JL, Moro A, Lu QY, Lu X, Li G, et al. The flavonoid quercetin inhibits pancreatic cancer growth in vitro and in vivo. Pancreas. 2013 Mar;42(2):223-9.

66. Neuhouser ML. Dietary flavonoids and cancer risk: evidence from human population studies. Nutr Cancer. 2004;50(1):1-7.

67. Grivennikov SI, Greten FR, Karin M. Immunity, inflammation, and cancer. Cell. 2010 Mar 19;140(6):883-99.

68. Prasad KN, Cole WC, Kumar B, Che Prasad K. Pros and cons of antioxidant use during radiation therapy. Cancer Treat Rev. 2002 Apr;28(2):79-91. 
69. Conklin KA. Cancer chemotherapy and antioxidants. J Nutr. 2004 Nov;134(11):3201S-4S.

70. Meierjohann S. Hypoxia-independent drivers of melanoma angiogenesis. Front Oncol. 2015;5:102.

71. Zbytek B, Peacock DL, Seagroves TN, Slominski A. Putative role of HIF transcriptional activity in melanocytes and melanoma biology. Dermatoendocrinol. 2013 Apr 01;5(2):239-51.

72. Ribatti D, Annese T, Longo V. Angiogenesis and melanoma. Cancers (Basel). 2010 Feb 25;2(1):114-32.

73. Vaupel P. The role of hypoxia-induced factors in tumor progression. Oncologist. 2004;9 Suppl 5:10-7.

74. Kipp AP, Deubel S, Arner ES, Johansson K. Time- and cell-resolved dynamics of redox-sensitive Nrf2, HIF and NF-kappaB activities in 3D spheroids enriched for cancer stem cells. Redox biology. 2017 Mar 10;12:403-9.

75. Yang J, Splittgerber R, Yull FE, Kantrow S, Ayers GD, Karin M, et al. Conditional ablation of Ikkb inhibits melanoma tumor development in mice. J Clin Invest. 2010 Jul;120(7):2563-74.

76. Qiao S, Lamore SD, Cabello CM, Lesson JL, Munoz-Rodriguez JL, Wondrak GT. Thiostrepton is an inducer of oxidative and proteotoxic stress that impairs viability of human melanoma cells but not primary melanocytes. Biochemical pharmacology. 2012 May 01;83(9):1229-40.

77. McNeely SC, Belshoff AC, Taylor BF, Fan TW, McCabe MJ, Jr., Pinhas AR, et al. Sensitivity to sodium arsenite in human melanoma cells depends upon susceptibility to arsenite-induced mitotic arrest. Toxicology and applied pharmacology. 2008 Jun 01;229(2):252-61.

78. Rocha CR, Kajitani GS, Quinet A, Fortunato RS, Menck CF. NRF2 and glutathione are key resistance mediators to temozolomide in glioma and melanoma cells. Oncotarget. $2016 \mathrm{Jul}$ 26;7(30):48081-92.

79. Liaudat AC, Bohl LP, Tolosa de Talamoni NG, Maletto B, Pistoresi-Palencia MC, Picotto G. Oxidative stress, cell cycle arrest and differentiation contribute toward the antiproliferative action of BSO and calcitriol on Caco-2 cells. Anticancer drugs. 2014 Aug;25(7):810-8.

80. Pronk TE, van der Veen JW, Vandebriel RJ, van Loveren $H$, de Vink EP, Pennings JL. Comparison of the molecular topologies of stress-activated 
transcription factors HSF1, AP-1, NRF2, and NF-kappaB in their induction kinetics of HMOX1. Bio Systems. 2014 Oct;124:75-85.

81. Dinkova-Kostova AT, Holtzclaw WD, Cole RN, Itoh K, Wakabayashi N, Katoh $\mathrm{Y}$, et al. Direct evidence that sulfhydryl groups of Keap1 are the sensors regulating induction of phase 2 enzymes that protect against carcinogens and oxidants. Proc Natl Acad Sci U S A. 2002 Sep 03;99(18):11908-13.

82. Kemp M, Go YM, Jones DP. Nonequilibrium thermodynamics of thiol/disulfide redox systems: a perspective on redox systems biology. Free radical biology \& medicine. 2008 Mar 15;44(6):921-37.

83. Flohe L. The fairytale of the GSSG/GSH redox potential. Biochimica et biophysica acta. 2013 May;1830(5):3139-42.

84. Spicer CD, Davis BG. Selective chemical protein modification. Nat Commun. 2014 Sep 05;5:4740.

85. Revesz L, Edgren MR, Wainson AA. Selective toxicity of buthionine sulfoximine (BSO) to melanoma cells in vitro and in vivo. Int J Radiat Oncol Biol Phys. 1994 May 15;29(2):403-6.

86. Dethlefsen LA, Lehman CM, Biaglow JE, Peck VM. Toxic effects of acute glutathione depletion by buthionine sulfoximine and dimethylfumarate on murine mammary carcinoma cells. Radiat Res. 1988 May;114(2):215-24.

87. Raposo G, Marks MS. Melanosomes--dark organelles enlighten endosomal membrane transport. Nat Rev Mol Cell Biol. 2007 Oct;8(10):786-97.

88. Lu J, Papp LV, Fang J, Rodriguez-Nieto S, Zhivotovsky B, Holmgren A. Inhibition of Mammalian thioredoxin reductase by some flavonoids: implications for myricetin and quercetin anticancer activity. Cancer Res. 2006 Apr 15;66(8):4410-8.

89. Sachweh MC, Stafford WC, Drummond CJ, McCarthy AR, Higgins M, Campbell J, et al. Redox effects and cytotoxic profiles of MJ25 and auranofin towards malignant melanoma cells. Oncotarget. 2015 Jun 30;6(18):16488-506.

90. Du Y, Zhang H, Lu J, Holmgren A. Glutathione and glutaredoxin act as a backup of human thioredoxin reductase 1 to reduce thioredoxin 1 preventing cell death by aurothioglucose. J Biol Chem. 2012 Nov 02;287(45):38210-9.

91. Lee SH, Meng XW, Flatten KS, Loegering DA, Kaufmann SH. Phosphatidylserine exposure during apoptosis reflects bidirectional trafficking between plasma membrane and cytoplasm. Cell Death Differ. 2013 Jan;20(1):6476. 
92. Chan FK, Moriwaki K, De Rosa MJ. Detection of necrosis by release of lactate dehydrogenase activity. Methods Mol Biol. 2013;979:65-70.

93. Jung DW, Kim WH, Seo S, Oh E, Yim SH, Ha HH, et al. Chemical targeting of GAPDH moonlighting function in cancer cells reveals its role in tubulin regulation. Chemistry \& biology. 2014 Nov 20;21(11):1533-45.

94. Potter M, Newport E, Morten KJ. The Warburg effect: 80 years on. Biochem Soc Trans. 2016 Oct 15;44(5):1499-505.

95. Xu XD, Shao SX, Jiang HP, Cao YW, Wang YH, Yang XC, et al. Warburg effect or reverse Warburg effect? A review of cancer metabolism. Oncol Res Treat. 2015;38(3):117-22.

96. Hanahan D, Weinberg RA. Hallmarks of cancer: the next generation. Cell. 2011 Mar 04;144(5):646-74.

97. Puzone R, Savarino G, Salvi S, Dal Bello MG, Barletta G, Genova C, et al. Glyceraldehyde-3-phosphate dehydrogenase gene over expression correlates with poor prognosis in non small cell lung cancer patients. Mol Cancer. 2013 Aug 29;12(1):97.

98. Zhu X, Huang C, Peng B. Overexpression of thioredoxin system proteins predicts poor prognosis in patients with squamous cell carcinoma of the tongue. Oral Oncol. 2011 Jul;47(7):609-14.

99. Ramos D, Pellin-Carcelen A, Agusti J, Murgui A, Jorda E, Pellin A, et al. Deregulation of glyceraldehyde-3-phosphate dehydrogenase expression during tumor progression of human cutaneous melanoma. Anticancer Res. 2015 Jan;35(1):439-44.

100. Hu XF, Yao J, Gao SG, Wang XS, Peng XQ, Yang YT, et al. Nrf2 overexpression predicts prognosis and 5-FU resistance in gastric cancer. Asian Pac J Cancer Prev. 2013;14(9):5231-5.

101. Wen J, You KR, Lee SY, Song CH, Kim DG. Oxidative stress-mediated apoptosis. The anticancer effect of the sesquiterpene lactone parthenolide. J Biol Chem. 2002 Oct 11;277(41):38954-64.

102. Pugazhenthi S, Nesterova A, Jambal P, Audesirk G, Kern M, Cabell L, et al. Oxidative stress-mediated down-regulation of bcl-2 promoter in hippocampal neurons. J Neurochem. 2003 Mar;84(5):982-96.

103. Sen N, Hara MR, Kornberg MD, Cascio MB, Bae BI, Shahani N, et al. Nitric oxide-induced nuclear GAPDH activates p300/CBP and mediates apoptosis. Nat Cell Biol. 2008 Jul;10(7):866-73. 
104. Colell A, Green DR, Ricci JE. Novel roles for GAPDH in cell death and carcinogenesis. Cell death and differentiation. 2009 Dec;16(12):1573-81.

105. Huang Q, Lan F, Zheng Z, Xie F, Han J, Dong L, et al. Akt2 kinase suppresses glyceraldehyde-3-phosphate dehydrogenase (GAPDH)-mediated apoptosis in ovarian cancer cells via phosphorylating GAPDH at threonine 237 and decreasing its nuclear translocation. J Biol Chem. 2011 Dec 09;286(49):42211-20.

106. Nakano K, Vousden KH. PUMA, a novel proapoptotic gene, is induced by p53. Mol Cell. 2001 Mar;7(3):683-94.

107. Vousden KH. p53: death star. Cell. 2000 Nov 22;103(5):691-4.

108. Basu A, Haldar S. The relationship between Bcl2, Bax and p53: consequences for cell cycle progression and cell death. Mol Hum Reprod. 1998 Dec;4(12):1099-109.

109. Yin HY, Wei JR, Zhang R, Ye XL, Zhu YF, Li WJ. Effect of glutamine on caspase-3 mRNA and protein expression in the myocardium of rats with sepsis. Am J Med Sci. 2014 Oct;348(4):315-8.

110. Galluzzi L, Blomgren K, Kroemer G. Mitochondrial membrane permeabilization in neuronal injury. Nat Rev Neurosci. 2009 Jul;10(7):481-94.

111. Zhu YZ, Zhu YC, Wang ZJ, Lu Q, Lee HS, Unger T. Time-dependent apoptotic development and pro-apoptotic genes expression in rat heart after myocardial infarction. Jpn J Pharmacol. 2001 Jul;86(3):355-8.

112. Rivlin N, Brosh R, Oren $M$, Rotter V. Mutations in the p53 Tumor Suppressor Gene: Important Milestones at the Various Steps of Tumorigenesis. Genes Cancer. 2011 Apr;2(4):466-74.

113. Tarze A, Deniaud A, Le Bras M, Maillier E, Molle D, Larochette N, et al. GAPDH, a novel regulator of the pro-apoptotic mitochondrial membrane permeabilization. Oncogene. 2007 Apr 19;26(18):2606-20.

114. Tait SW, Green DR. Mitochondria and cell death: outer membrane permeabilization and beyond. Nat Rev Mol Cell Biol. 2010 Sep;11(9):621-32.

115. Galadari S, Rahman A, Pallichankandy S, Thayyullathil F. Reactive oxygen species and cancer paradox: To promote or to suppress? Free radical biology \& medicine. 2017 Mar;104:144-64.

116. Vakkila J, Lotze MT. Inflammation and necrosis promote tumour growth. Nat Rev Immunol. 2004 Aug;4(8):641-8.

117. Lotze MT, Demarco RA. Dying dangerously: Necrotic cell death and chronic inflammation promote tumor growth. Discov Med. 2004 Dec;4(24):448-56. 

Chapter 8

Summary and general discussion 


\section{Summary and general discussion}

\subsection{Hormesis}

The environment is always changing and life on earth has to adapt to the challenges. For example, when oxygen was introduced in the atmosphere, organisms had to adapt by constructing an antioxidant network to cope with oxygen toxicity. One of the adaptive processes is hormesis. In hormesis the adaptive or compensatory response is induced by a relatively subtle challenge of homeostasis by a toxic compound (1-3). Over time many terms have been given to the same phenomenon, the most recent one is from Davies, adaptive homeostasis, which is a physiological adaptation that leads to a (transient) change or expansion of the internal steady state or (transient) disturbance of the homeostatic balance (1).

\subsection{Time and adaptation}

The induction of an adaptive response is dependent on various factors (Chapter 2). A lot of factors are important to ensure that an adaptive response is induced (3). First of all, the intrinsic reactivity of a compound is crucial. Some compounds are more potent to induce hormesis than others. Second, a relatively low dose is necessary to induce an adaptive response that protects against a toxic dose of the same compound. Notably, what a low dose is, depends on the compound. Compounds are regarded as hazardous when they elicit a harmful response in a relatively low concentration. It seems that this also applies for the adaptive response. For example, for the hazardous acrolein a low dose of $0.15 \mu \mathrm{g} / \mathrm{cm}^{2}$, a concentration lower than $10 \mu \mathrm{M}$, already induces $50 \%$ toxicity in A549 cells, whereas for the "relatively safe" silver nanoparticles (AgNPs) a much higher dose of at least $55 \mu \mathrm{g} / \mathrm{cm}^{2}$, depending on the physicochemical properties, is needed to achieve a similar effect (4) (Chapter 4). Besides the intrinsic reactivity of the compound, also the physicochemical nature of the compounds is involved, acrolein is water soluble and is taken up by cells by passive diffusion, while AgNPs form agglomerates and are taken up by endocytosis and slowly released in the cell's cytoplasm.

Besides the nature of the compound and the dose, time is also crucial in adaptation (Chapter 2). An organism often needs time to adapt to a change such as exposure to a toxin. If the duration of exposure is too short or the frequency of exposure too high, there is no time for the adaptive response to be induced. The nature of the adaptive response also determines the time needed. Direct enzyme modification or cofactor regulations are the quickest adaptive responses. Depending on the oxygen level, associated oxidative stress and concomitant glutathione (GSH) levels, the activity of the 
rate-limiting enzyme of GSH synthesis can be regulated. Adaptations that need more time are for example the transcriptional increase of the endogenous antioxidant systems including nuclear factor (erythroid-derived 2)-like 2 (Nrf2)-mediated expression of enzymes involved in GSH synthesis. In the long run, adaptations at the epigenetic and genomic level should be considered; for example, the ability to synthesize GSH by phototrophic bacteria. All these forms of adaptation are encoded in the genome. Although, if for an individual an adaptive response is induced too early or too late, this can be detrimental on a short term, because it costs a lot of energy. Apparently, the ability to adapt is evolutionary beneficial.

\subsection{Redox signaling in adaptation to acrolein and nanoparticles}

In human bronchial epithelial adenovirus 12-SV40 infected (BEAS-2B) cells, GSH is the first line of defense against acrolein (5) (Chapter 3). If BEAS-2B cells are exposed to a concentration lower than $3 \mu \mathrm{M}$, no cytotoxicity is induced. At these low concentrations, GSH reacts in a stoichiometry of 1:1 with acrolein. At a higher concentration of $10 \mu \mathrm{M}, 80 \%$ of the acrolein does not react with GSH and is free to bind with other cellular targets finally resulting in cell death. This confirms the paradigm that at low doses of acrolein the cells are protected and the GSH system is the main protective mechanism, whereas at high doses toxicity is induced. Next to this, we also showed that acrolein can induce adaptation. Pretreatment of BEAS-2B cells with a relatively low and non-toxic concentration of $3 \mu \mathrm{M}$, increases the gene expression of Nrf2-mediated $\gamma$ glutamylcysteine synthetase ( $\gamma \mathrm{GCS}$ ) leading to elevated GSH levels. This protects against toxicity due to exposure to a relatively high toxic concentration of acrolein of $10 \mu \mathrm{M} 3$ hours later. Both the GSH protection and adaptation via the Kelch-like $\mathrm{ECH}$-associated protein 1 (Keap1)-Nrf2 pathway are induced via a thiol reactive mechanism. The $\alpha, \beta-$ unsaturated aldehyde acrolein prefers to react via 1,4 Michael addition with thiols of first of all GSH, but at higher concentrations with thiols present in other proteins, for example the thiol present in cysteine 288 of Keap1 (6). Notably, the characteristic structure of acrolein can also be found in other molecules, therefore acrolein is defined as a toxicophore. It functions as a model for electrophilic xenobiotics that display toxicity as a direct result of their electrophilicity, e.g. N-acetyl-p-benzoquinone imine (NAPQI) (7), a byproduct from the metabolism of acetaminophen or paracetamol.

Acrolein is not the only compound able to induce adaptation. In this thesis, we showed that also silver nanoparticles can induce hormesis in A549 cells (4) (Chapter 4). In this study we investigated two types of AgNPs, the first one is AgNP1 and has the biggest size of $37 \mathrm{~nm}$, but a lower solubility. The second one AgNP2 has a size of approximately $16.6 \mathrm{~nm}$ and is much better soluble. In addition, the AgNP2 has a greater tendency to 
agglomerate, whereas the shape of the AgNP2 is more spherical. The toxicity of AgNP2 appeared to be higher than for AgNP1 and all their physicochemical properties should be taken into account when explaining their difference in toxicity. Both types of silver nanoparticles and acrolein induced adaptation. For AgNP1 and AgNP2, pretreatment with 2.5 or $5 \mu \mathrm{g} / \mathrm{cm}^{2}$, respectively, resulted in less toxicity induced by 60 or $80 \mu \mathrm{g} / \mathrm{cm}^{2}$, respectively, 24 hours later. For acrolein, adaptation was induced by $10 \mu \mathrm{M}$ acrolein that protected against toxicity of $100 \mu \mathrm{M}$ acrolein 4 hours later. Remarkably, A549 cells are more resistant against acrolein toxicity than BEAS-2B cells. The difference in cellular sensitivity is also apparent for other compounds including multi-walled carbon nanotubes (MWCNTs) (8). Furthermore, in A549 cells more Nrf2-mediated upregulation has been seen. Apparently, also the endogenous antioxidant system seems to be different between the two different cell types. Remarkably, the low concentration of acrolein seems to increase GSH levels, increase Nrf2 translocation and increase the expression of endogenous antioxidant genes $\gamma \mathrm{GCS}$ and heme oxygenase-1 (HO-1). For both AgNPs, no effect on GSH levels and gene expression of $\gamma$ GCS was seen, while nuclear presence of Nrf2 and HO-1 gene expression were increased. On the other hand, pretreatment with a low dose of the AgNPs, $2.5 \mu \mathrm{g} / \mathrm{cm}^{2}$ for AgNP1 and $5 \mu \mathrm{g} / \mathrm{cm}^{2}$ for AgNP2, protected against toxicity of a high dose of $100 \mu \mathrm{M}$ of acrolein. This indicates that AgNPs can induce an adaptive response that apart from protection against a challenge with the compound itself can also protect against a challenge with another compound, in this case acrolein, a process called transhormesis. This can only be explained, when the same adaptive response is induced to induce protection despite of the differences found in the mechanism. The mechanism of how AgNPs induce toxicity is still unknown, although some studies show that they increase reactive oxygen species (ROS) (9).

\subsection{Antioxidant flavonoids, protection and mechanism of adaptation}

Flavonoids are polyphenolic compounds present in fruit and vegetables. In this thesis the most abundant natural flavonoids rutin and quercetin are studied. They are able to scavenge ROS, thereby functioning as an antioxidant. During this scavenging they are converted to their oxidized form, called quinone. Due to this antioxidant activity, they protect against the damaging effects of ROS. If ROS are not scavenged, this will lead to oxidative damage to deoxyribonucleic acid (DNA), proteins or lipids (10). However, the quinone form of the flavonoid has been shown to be thiol reactive. Previously, it has been shown that dependent on its structure it prefers to direct its reactivity towards either GSH or vitamin $\mathrm{C}$ in the endogenous antioxidant network (11). Due to its rutinose group, rutin is a much harder electrophile than quercetin. It is thought that rutin quinone probably prefers to react with vitamin C over GSH, because vitamin $\mathrm{C}$ is also a much harder nucleophile. According to the Hard Soft Acid Base (HSAB) concept, hard electrophiles 
prefer to react with hard nucleophiles and soft electrophiles with soft nucleophiles. GSH and vitamin $\mathrm{C}$ are not the only members of the endogenous antioxidant network. In this thesis in Chapter 5, we confirmed that rutin functions as an antioxidant decreasing the endogenous level of oxidative stress. The formed rutin quinone can react with thiols present in Keap1 and decrease the activity of the endogenous antioxidant enzyme thioredoxin reductase 1 (TrxR1). This will subsequently lead to an increased Nrf2 activity. In addition, an increased glutamate cysteine ligase (GCL) gene expression was measured in human umbilical vein endothelial cells (HUVECS). Next to that, oxidative stress increases proinflammatory nuclear factor kappa-light-chain-enhancer of activated B cells (NF-kB) activity and pro-angiogenesis hypoxia-inducible factor (HIF) activity. However, rutin quinone is able to blunt this effect. Previously, it was seen that an upregulation of GCL also results in increased GSH levels. This probably explains the adaptive response seen for rutin quinone. And it should be noted that this is a targeted response, since it only happens in regions with oxidative stress. Addition of rutin quinone, 3 hours before a second challenge with oxidative stress, resulted in diminished oxidative stress compared with the condition without rutin quinone pretreatment. This phenomenon of a defined non-harmful compound inducing an adaptive response against a toxic compound, in this case oxidative stress, is called parahormesis $(12,13)$. To illustrate the physiological relevance of this finding, the effect on the local vasomotor response in vessels was determined. From oxidative stress, it is known that it can impair vasorelaxation of arterioles (14), which was confirmed in secondary human placental arterioles. Pretreatment with rutin protected against oxidative stress-triggered impaired vasorelaxation of human placental arterioles. This can be attributed, firstly, to its direct antioxidant scavenging capacity. However, due to its short half-life the protective effects against development of cardiovascular disease (CVD) cannot be explained. However, the increase in Nrf2-mediated endogenous antioxidants by rutin quinone, can explain the protective effects on a longer time scale. Regarding the vasomotor responses, thiol supplementation has been shown to increase nitric oxide (NO) production. In addition, a previous study, showed that rutin in presence of hydrogen peroxide $\left(\mathrm{H}_{2} \mathrm{O}_{2}\right)$ could also increase endothelial nitric oxide synthase (eNOS) messenger ribonucleic acid (mRNA) (15) and probably by this way rutin quinone and not rutin can enhance the vasodilatory effects in blood vessels.

\subsection{Thiol reactivity and adaptation versus amino reactivity and toxicity}

Acrolein, AgNPs and rutin quinone can induce adaptation at low concentrations and exposure times. For acrolein also the effects of high concentrations and increased incubation times were determined (Chapter 6). At low concentrations and short incubation times, GSH can protect against damage induced by acrolein. An increased concentration of acrolein and a longer incubation with acrolein activated microsomal 
glutathione-S-transferase 1 (MGST1). Acrolein reacts via 1,4 Michael addition with the thiol in cysteine 49. If concentrations of acrolein are too high or longer incubation times are used, next to the thiols also amino groups of MGST1 can be targeted. Targeting of the amino groups results in the formation of a Schiff base or thiazolidine derivative that inhibits MGST1 activity. This indicates that first GSH protects against the damaging effects of acrolein. By activating MGST1, a short term adaptive mechanism is induced. Finally, at too high concentrations or too long incubation times, acrolein overwhelms the cellular systems and can attack vital cellular targets including essential proteins or DNA. This will damage the cell and enhance various oxidative stress-related diseases. In order to protect the cell against this development, it would be beneficial to decrease endogenous protection by inhibiting MGST1 and enhance programmed cell death. This last mechanism is not substantiated in Chapter 6, but will be explained in more detail in the next paragraph.

\subsection{Apoptosis, a mechanism of toxicity or protection?}

Next to rutin quinone also quercetin quinone can induce adaptation by increasing Nrf2 activity. In addition, quercetin quinone decreases NF-KB and HIF activity induced by oxidative stress.

Remarkably, in tumor cells, in this case GSH-depleted A375 melanoma cells, quercetin quinone decreases Nrf2-mediated expression of endogenous antioxidant genes (Chapter 7). In addition, also the activity of TrxR1 is decreased. At the same time, quercetin quinone induces apoptosis, while oxidative stress results in necrosis. Additionally, glyceraldehyde-3-phosphate dehydrogenase (GAPDH) nuclear translocation is increased, which coincides with a trend towards increased ratio of pro-apoptotic versus anti-apoptotic gene expression (Chapter 7). A375 melanoma cells that are simultaneously exposed to high oxidative stress and quercetin show an inhibition of Nrf2-mediated adaptation and shift cell death from oxidative stress-induced necrosis to apoptosis. This indicates that, if a cell is exposed to high levels of oxidative stress, in this case a tumor cell, instead of protecting a cell undergoing a lot of damage to essential proteins or DNA, programmed cell death or apoptosis is induced. This is of course toxic for the cell, but protects the living organism against the detrimental effects of damaged, not properly functioning, cells. This is again a targeted approach, since quercetin is only converted into quercetin quinone in regions with oxidative stress and for example in tumor cells Nrf2, TrxR1 and GAPDH are upregulated. 


\section{Implications, limitations and recommendations for future research}

More understanding of our ability to adapt, and especially in the context of time, will enhance our understanding of how organisms are able to adapt their intricate redox signaling. With this knowledge, we can intervene in this process and at the right time enhance adaptation to ensure that organisms can better cope with encountered changes in the environment. They are better protected against oxidative challenges disturbing their internal redox balance and thereby less likely to develop associated oxidative stressrelated diseases like chronic obstructive pulmonary disease (COPD), CVD and cancer. We described the current knowledge about the concept of time in adaptive processes. This knowledge is however not complete, especially, regarding adaptive processes on a long term knowledge is lacking. For example, are we able to limitless induce adaptive processes or are we getting used to the exposure at a certain time and does our ability to adapt fade out, and does a kind of desensitization occur? Additionally, also the mechanisms of adaptation seem to be similar for some chemicals, e.g. acrolein and silver nanoparticles, but which chemicals can induce an adaptive response that also protect against each other? Is this for all chemicals similar or do different compounds induce different mechanisms of adaptation and are they then still able to induce transhormesis? Both questions need further investigation. In addition, the concept of hormesis is currently not included in risk assessment legislation of toxic compounds. It could be investigated whether we need to increase our threshold limit value (THV) or acceptable daily intake (ADI) for compounds, to make sure that adaptive processes are included to protect against future exposures. The major limitation of this process is however, that inducing adaptation costs energy and this energy is spilled if we are not exposed to a future challenge. However, we cannot always predict when we will be exposed to a future challenge.

As the mechanism of adaptation for acrolein and AgNPs is found to be similar, it remains enigmatic what is causing this adaptation. In order to determine whether in both cases a Nrf2-mediated mechanism causes these adaptations induced by acrolein or AgNPs, a Nrf2 knock out study should be performed. Furthermore, the finding that not all Nrf2mediated genes are activated by the investigated compounds, can be due to the fact that either not all coactivators are present for the specific gene, also other transcription factors are involved in up- or downregulation of this specific gene or epigenetic changes are induced. Also timing can play an important role, since GSH seems to be more important in the more short term responses to acrolein, whereas due to long term exposures to AgNPs also HO-1 gene expression is induced. It is still questioned whether hormesis and 
transhormesis are also applicable for other nanoparticles (NPs) including titanium dioxide $\left(\mathrm{TiO}_{2}\right)$ or gold nanoparticles (AuNPs).

Also rutin quinone can induce an adaptive response by increasing expression of Nrf2-mediated endogenous antioxidants. The effects of the rutin quinone have only been shown indirectly. Again, with a Nrf2 knock out model, the causal effect of Nrf2 could be investigated. Additionally, it is recommended to show rutin quinone formation and adduct formation on specific proteins e.g. Keap1 intracellular. Previous attempts to find these adducts, encountered difficulties regarding low expression of Keap1 in the examined cell types or low stability with regard to the formed adduct. Keap1 overexpression and nonreducing gels are recommended to be used in future experiments. Furthermore, it is recommended to investigate adaptation in blood vessels, by preincubating them with rutin quinone and over time investigating the vasomotor responses after an exposure to oxidative stress a few hours later. Also, it would be interesting to elaborate the found effect for rutin quinone, by investigating endothelial monolayer integrity (vascular leakage) by measuring the electrical resistance using Electric Cell-substrate Impedance Sensing (ECIS) (16). This could link the redox-induced vascular effects with inflammation. However, it should be noted that rutin quinone probably more selectively targets proteins in the cell than the damaging ROS. One of these targets could be Keap1, which acts as a sensor for specific compounds. Acrolein has been shown to selectively target cysteine 288 , just like other alkenal-like substrates as 4-hydroxy-2-nonenal, whereas rutin quinone binds to cysteine 151 (6). Cysteine 288 is present in the intervening region, probably resulting in a conformational change impairing interaction with Nrf2, while cysteine 151 is located in the Bric-à-Brac (BTB) domain of Keap1 that is involved in the homodimerization of the Keap1 dimer and binding to cytoplasmic cytoskeleton (17). In both cases, adduct formation interferes with Keap1's interaction with Nrf2 and influences the downstream activation of endogenous antioxidant genes. But the subset of cysteines that is attacked in Keap1 is probably compound-dependent. Next to the effects on Keap1, also the GSH level could be determined over time and the resulting effect on NO production due to rutin quinone should be confirmed. Finally, the newly found mechanism behind the long term protection of rutin against CVD, the selective upregulation of endogenous antioxidant systems, can in the future contribute to the further understanding why rutin can reduce the risk on developing cardiovascular disease. Additionally, the induction of adaptation could also be examined for other flavonoids, with a small difference in their structure. It could be questioned whether this difference in hardness, leads to more potent adaptation and more stable adducts with Keap1.

Regarding the acrolein modification of MGST1, it could be questioned whether it is indeed only the amino group binding that leads to inhibition of MGST1. Capping of 
amino reactive groups before acrolein treatment at high concentrations or long incubation times could answer this question. In addition, amino reactive compounds can confirm that binding to amino groups leads to MGST1 inhibition. Finally, the specific amino groupmediated inhibition of MGST1 should be validated in tumor cells to investigate the protection against established anticancer drug resistance by inhibiting MGST1 capacity of detoxifying anticancer drugs. Maybe in this case, acrolein is not a good compound to use, but flavonoid quinones could be considered. With regard to this, the dietary flavonoids function as a therapeutic and can be seen as nutraceutical.

Regarding the apoptosis-inducing effects of quercetin quinone, the causal effect of GAPDH inducing apoptosis could be investigated in a (temporarily) nuclear GAPDH knock out model. In addition, the GAPDH-quercetin adduct should be examined intracellular.

Finally, there are some indications that these hormetic responses occur in an in vivo setting. For example chronic smokers have higher GSH levels in their lung epithelial lining fluid than non-smokers or acute smokers (18). However, it should be confirmed that exposure to a low dose of a specific compound protects against a second challenge with the same or another compound. The risk assessment might change as a result of this. The societal impact will undoubtedly raise some ethical concerns.

\section{Conclusion}

The paradigm Paracelsus has evolved over time (Figure 1). Looking back he suggested in 1500: 'Sola dosis facit venenum' (in English: The dose makes the poison), meaning that every compound or toxin will be toxic, depending on the dose. At a low dose, defense systems in our body can offer protection for example the redox systems GSH and thioredoxin (Trx). At a dose that is too high, the protection systems are overwhelmed and the compound will induce toxic effects. In this thesis we refined the paradigm of Paracelsus by adding an additional level to the continuum between protection and toxicity: the level of adaptation. If the dose of the chemical is approaching the border of toxicity, the protection systems are not $100 \%$ full proof, and the toxin selectively targets specific pathways that induce adaptation and contribute to an enhanced protection on a longer term, for example a transcriptional increase of endogenous antioxidant enzymes. That is the current paradigm, which is also illustrated by some of the research in this thesis. This paradigm will further develop over time. We expect that in the future, probably additional levels and sublevels will be defined. For example the level of toxicity can already be subdivided in apoptosis and necrosis. Some compounds enhance apoptotic processes e.g. 
quercetin quinone, whereas others induce necrosis for example $\mathrm{H}_{2} \mathrm{O}_{2}$ that contributes to oxidative stress.

The Janus face of Paracelsus

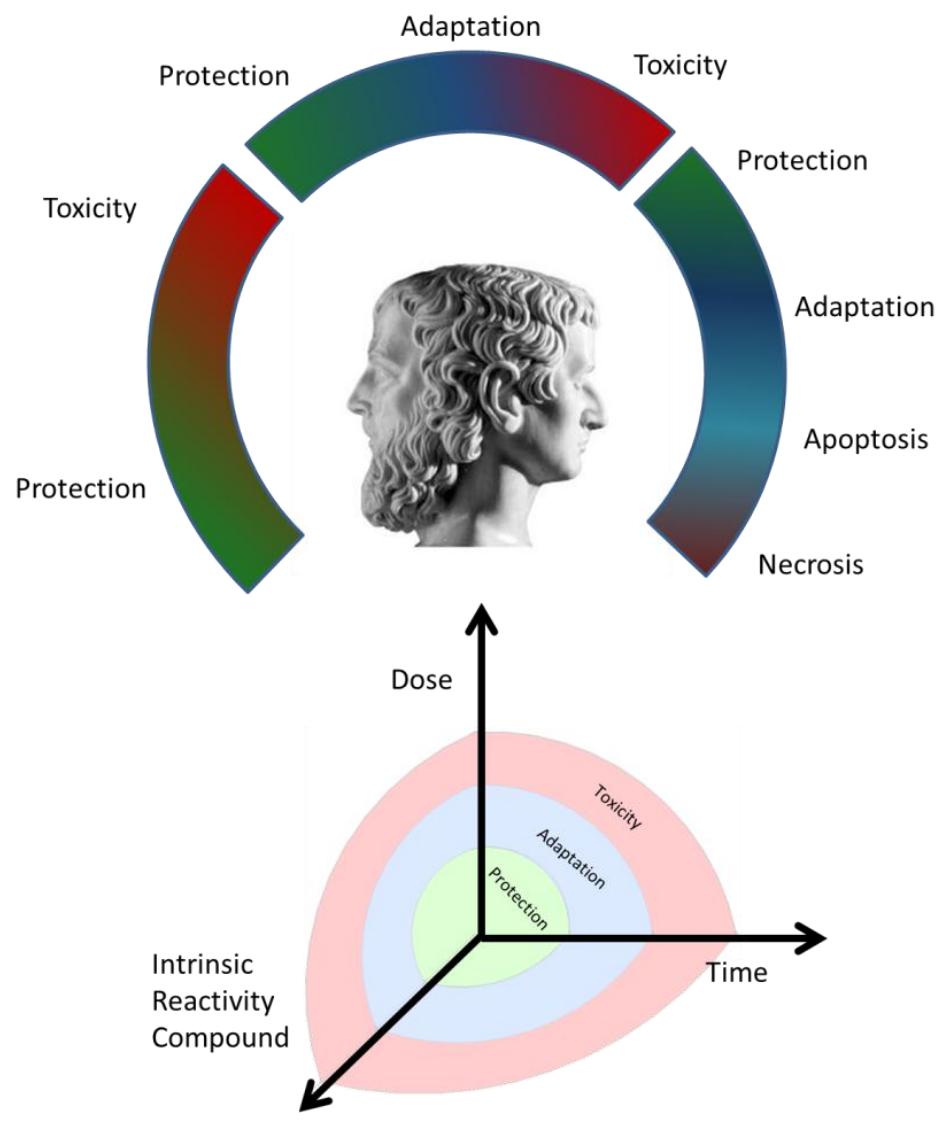

Figure 1: The Janus face of Paracelsus: the paradigm of Paracelsus, "Sola dosis facit venenum", evolved over time, taken into account dose, time and the intrinsic reactivity of the compound of interest.

Also another dimension might be added that relates to the reactivity of compounds. The nature of the compound of interest is a dimension that should be taken into account in this paradigm. One of the strategies in toxicology to protect is to neutralize the reactivity of the toxin of interest. For example, initially it was attempted to completely annihilate the reactivity of ROS by an antioxidant. While in the current paradigm, reactivity can be selectively directed. For example, the reactivity of ROS is not only 
annihilated by an antioxidant like quercetin, but quercetin is converted into quercetin quinone by ROS that can selectively induce adaptation by upregulation of the endogenous antioxidant systems to protect in the future against the damaging ROS. Depending on the circumstances, adaptation or toxicity can be regarded as beneficial or detrimental. Enhancing adaptation by increasing the endogenous antioxidant response in a tumor is not desirable, whereas toxicity, especially induction of apoptosis, could be advantageous in a cancer cell. However, how this paradigm will evolve in the upcoming future and what we could do to intervene in these processes will need to be seen. Tempus omnia revelat. 


\section{References}

1. Davies KJ. Adaptive homeostasis. Mol Aspects Med. 2016 Jun;49:1-7.

2. Mattson MP. Hormesis defined. Ageing research reviews. 2008 Jan;7(1):1-

7.

3. Sthijns MM, Weseler AR, Bast A, Haenen GR. Time in Redox Adaptation Processes: From Evolution to Hormesis. International journal of molecular sciences. 2016 Sep 29;17(10).

4. Sthijns MM, Thongkam W, Albrecht C, Hellack B, Bast A, Haenen GR, et al. Silver nanoparticles induce hormesis in $A 549$ human epithelial cells. Toxicology in vitro : an international journal published in association with BIBRA. 2017 Jan 18.

5. Sthijns MM, Randall MJ, Bast A, Haenen GR. Adaptation to acrolein through upregulating the protection by glutathione in human bronchial epithelial cells: the materialization of the hormesis concept. Biochemical and biophysical research communications. 2014 Apr 18;446(4):1029-34.

6. McMahon M, Lamont DJ, Beattie KA, Hayes JD. Keap1 perceives stress via three sensors for the endogenous signaling molecules nitric oxide, zinc, and alkenals. Proc Natl Acad Sci U S A. 2010 Nov 02;107(44):18838-43.

7. Nassini R, Materazzi S, Andre E, Sartiani L, Aldini G, Trevisani M, et al. Acetaminophen, via its reactive metabolite $\mathrm{N}$-acetyl-p-benzo-quinoneimine and transient receptor potential ankyrin-1 stimulation, causes neurogenic inflammation in the airways and other tissues in rodents. FASEB journal : official publication of the Federation of American Societies for Experimental Biology. 2010 Dec;24(12):4904-16.

8. Ursini CL, Cavallo D, Fresegna AM, Ciervo A, Maiello R, Buresti G, et al. Differences in cytotoxic, genotoxic, and inflammatory response of bronchial and alveolar human lung epithelial cells to pristine and $\mathrm{COOH}$-functionalized multiwalled carbon nanotubes. Biomed Res Int. 2014;2014:359506.

9. Kermanizadeh A, Vranic S, Boland S, Moreau K, Baeza-Squiban A, Gaiser $B K$, et al. An in vitro assessment of panel of engineered nanomaterials using a human renal cell line: cytotoxicity, pro-inflammatory response, oxidative stress and genotoxicity. BMC Nephrol. 2013 Apr 25;14:96.

10. Tabak O, Gelisgen R, Erman H, Erdenen F, Muderrisoglu C, Aral H, et al. Oxidative lipid, protein, and DNA damage as oxidative stress markers in vascular complications of diabetes mellitus. Clin Invest Med. 2011 Jun 01;34(3):E163-71. 
11. Jacobs $H$, Moalin M, Bast A, van der Vijgh WJ, Haenen GR. An essential difference between the flavonoids monoHER and quercetin in their interplay with the endogenous antioxidant network. PLoS One. 2010 Nov 08;5(11):e13880.

12. Ursini F, Maiorino M, Forman HJ. Redox homeostasis: The Golden Mean of healthy living. Redox biology. 2016 Aug;8:205-15.

13. Forman HJ, Davies KJ, Ursini F. How do nutritional antioxidants really work: nucleophilic tone and para-hormesis versus free radical scavenging in vivo. Free radical biology \& medicine. 2014 Jan;66:24-35.

14. Thengchaisri N, Hein TW, Wang W, Xu X, Li Z, Fossum TW, et al. Upregulation of arginase by $\mathrm{H} 2 \mathrm{O} 2$ impairs endothelium-dependent nitric oxidemediated dilation of coronary arterioles. Arteriosclerosis, thrombosis, and vascular biology. 2006 Sep;26(9):2035-42.

15. Ugusman A, Zakaria Z, Chua KH, Nordin NA, Abdullah Mahdy Z. Role of rutin on nitric oxide synthesis in human umbilical vein endothelial cells. ScientificWorldJournal. 2014;2014:169370.

16. van Wetering S, van Buul JD, Quik S, Mul FP, Anthony EC, ten Klooster JP, et al. Reactive oxygen species mediate Rac-induced loss of cell-cell adhesion in primary human endothelial cells. J Cell Sci. 2002 May 01;115(Pt 9):1837-46.

17. Kansanen E, Kuosmanen SM, Leinonen H, Levonen AL. The Keap1-Nrf2 pathway: Mechanisms of activation and dysregulation in cancer. Redox biology. 2013 Jan 18;1:45-9.

18. Rahman I, MacNee W. Lung glutathione and oxidative stress: implications in cigarette smoke-induced airway disease. Am J Physiol. 1999 Dec;277(6 Pt 1):L1067-88. 

Chapter 9

Nederlandse samenvatting en algemene discussie 


\section{Nederlandse samenvatting en algemene discussie}

\subsection{Hormesis}

De omgeving is altijd aan verandering onderhevig en het leven op aarde moet zich voortdurend aanpassen aan deze veranderingen. Bijvoorbeeld toen zuurstof voor het eerst in de atmosfeer kwam, moesten organismen zich aanpassen door een antioxidant netwerk te creëren dat beschermt tegen deze zuurstof toxiciteit. Een van deze adaptieve processen heet hormesis. Tijdens hormesis wordt een adaptieve of compensatoire respons geïnduceerd door een relatief subtiele verstoring van homeostase met een toxische stof. (1-3). Er bestaan verschillende termen voor het concept hormesis. De meest recente is gedefinieërd door Davies. Davies suggereerde de term adaptieve homeostase. Dit is een fysiologische adaptatie die resulteert in een (tijdelijke) verandering of uitbreiding van het interne evenwicht of homeostase (1).

\subsection{Tijd en aanpassing}

Verschillende factoren kunnen het induceren van adaptatie beïnvloeden (3) (Hoofdstuk 2). Allereerst, is de intrinsieke reactiviteit van een stof cruciaal. Sommige stoffen zijn beter in het induceren van adaptatie dan andere stoffen. Ten tweede, is de dosis belangrijk. Een relatief lage dosis is nodig om adaptatie te induceren. Door zich aan te passen is het organisme vervolgens beschermd tegen blootstelling met een toxische dosis van de desbetreffende stof. Notabene, wat gedefinieërd wordt als een lage dosis, is afhankelijk van de desbetreffende stof. Van toxische stoffen is er absoluut een lagere concentratie nodig om adaptatie te induceren. Bijvoorbeeld, acroleine, een stof in sigarettenrook, induceert al $50 \%$ toxiciteit in A549 cellen bij een concentratie van 0.15 $\mu \mathrm{g} / \mathrm{cm}^{2}$ of $10 \mu \mathrm{M}$. Terwijl zilver nanodeeltjes (AgNPs), afhankelijk van hun fysischchemische eigenschappen, pas bij een concentratie van $55 \mu \mathrm{g} / \mathrm{cm}^{2}$ een vergelijkbaar effect geven (4) (Hoofdstuk 4). Naast de intrinsieke reactiviteit van de stof, zijn ook de fysischchemische eigenschappen betrokken. Acroleine is wateroplosbaar en wordt opgenomen in de cel via passieve diffusie, terwijl AgNPs kunnen agglomereren en opgenomen worden via endocytose en pas langzaam vrijkomen in het cytoplasma.

Behalve de aard van de stof en de dosis, is ook de tijd cruciaal tijdens adaptatie (Hoofdstuk 2). Een organisme heeft tijd nodig om zich te kunnen aanpassen aan een verandering, bijvoorbeeld aan de blootstelling aan een toxische stof. Als de blootstellingsduur te lang is of de frequentie van de blootstellingen te hoog, dan is er geen tijd om de adaptieve respons te induceren. De aard van de adaptieve respons bepaalt hoeveel tijd er nodig is om de adaptatie te kunnen doen. De snelste adaptieve responsen 
zijn directe enzymmodificaties en cofactor regulaties. Het snelheidsbepalende enzym van de glutathion (GSH) synthese wordt gereguleerd door de zuurstofspanning, geassocieerde oxidatieve stress en daarmee gepaard gaande GSH concentraties. Adaptaties waar meer tijd voor nodig is, zijn bijvoorbeeld een endogene toename in antioxidantsystemen op transcriptie niveau zoals nuclear factor (erythroid-derived 2)-like 2 (Nrf2)-gemedieerde expressie van enzymen betrokken bij de GSH synthese. Op lange termijn zijn adaptaties met uitwerkingen op epigenetisch en genetisch niveau van belang; zoals de mogelijkheid van fototrofe bacteriën om GSH te synthetiseren. Al deze vormen van adaptatie liggen opgeslagen in het genoom. Voor een individu kan een adaptieve respons te vroeg of te laat zijn, met schadelijke effecten op de korte termijn, omdat veel energie wordt verbruikt. Echter de mogelijkheid om je aan te passen blijkt evolutionair voordelig.

\subsection{Redox signalering in adaptatie tegen acroleine en nanodeeltjes}

In humane bronchiale epitheliale adenovirus 12-SV40 geïnfecteerde (BEAS-2B) cellen, vormt GSH de eerste verdediging tegen acroleine (5) (Hoofdstuk 3). Een concentratie van acroleine lager dan $3 \mu \mathrm{M}$ geeft dan ook geen cytotoxiciteit, omdat GSH in een stoïchiometrie van 1:1 met acroleine reageert. Echter bij een concentratie van 10 $\mu \mathrm{M}$ acroleine, reageert $80 \%$ van het acroleine niet met GSH, maar met andere cellulaire onderdelen zoals proteïnen, hetgeen uiteindelijk resulteert in celdood. Dit bevestigt het paradigma dat cellen beschermd zijn tegen lage dosissen van acroleine en dat GSH het belangrijkste beschermingsmechanisme is, terwijl een hoge dosis aan acroleine tot toxiciteit leidt. Daarnaast, hebben we ook laten zien dat acroleine tot adaptatie kan leiden. Voorbehandeling van BEAS-2B cellen met een relatief lage niet toxische concentratie acroleine van $3 \mu \mathrm{M}$ resulteert in een toename in gen expressie van Nrf2-gemedieerde $\gamma$ glutamylcysteine synthetase $(\gamma \mathrm{GCS})$ dat de GSH levels laat stijgen. Dit beschermt vervolgens tegen de toxiciteit als gevolg van de blootstelling aan een relatieve hoge concentratie aan acroleine van $10 \mu \mathrm{M} 3$ uur later. Zowel de bescherming door GSH als de adaptatie via de Kelch-like ECH-associated protein 1 (Keap1)-Nrf2 pathway worden geïnduceerd via een thiol reactief mechanisme. Het $\alpha, \beta$ onverzadigde aldehyde acroleine reageert met thiolen zoals GSH via een 1,4 Michaël additie. Allereerst reageert acroleine met GSH, maar bij hogere concentraties aan acroleine zullen ook andere thiolen zoals het thiol in cysteïne 288 van Keap1 reageren (6). Daarbij komt nog dat de karakteristieke structuur van acroleine ook herkenbaar is in de structuur van andere moleculen. Daarom wordt acroleine ook wel een toxicofoor genoemd. Het functioneert als een model stof voor electrofiele xenobiotica die toxisch zijn vanwege hun electrofiele eigenschappen. Een voorbeeld hiervan is $\mathrm{N}$-acetyl-p-benzoquinone imine (NAPQI) (7), een metaboliet in het metabolisme van paracetamol. 
Acroleine is niet de enige stof die adaptatie kan induceren. In deze thesis hebben we laten zien dat ook AgNPs hormesis kunnen induceren in A549 cellen (4) (Hoofdstuk 4). In deze studie, hebben we twee verschillende types AgNPs onderzocht. De eerste is AgNP1, die met $37 \mathrm{~nm}$ het grootste is en een lage oplosbaarheid heeft. De tweede is AgNP2. Dit nanodeeltje is $16.6 \mathrm{~nm}$ groot en heeft een veel betere oplosbaarheid dan AgNP1. Daar komt nog bij dat AgNP2 een grotere neiging tot agglomeratie heeft en een rondere vorm heeft. De toxiciteit van AgNP2 is groter dan de toxiciteit van AgNP1. Daarom moeten ook alle fysisch-chemische eigenschappen worden meegenomen om het verschil in toxiciteit te verklaren. Zowel beide types AgNPs als acroleine induceren adaptatie. Voorbehandeling met $2.5 \mu \mathrm{g} / \mathrm{cm}^{2}$ AgNP1 of $5 \mu \mathrm{g} / \mathrm{cm}^{2}$ AgNP2 leidt ertoe dat $60 \mu \mathrm{g} / \mathrm{cm}^{2}$ AgNP1 of $80 \mu \mathrm{g} / \mathrm{cm}^{2}$ AgNP2 24 uur later minder toxisch is dan zonder voorbehandeling. Hetzelfde geldt voor acroleine: $10 \mu \mathrm{M}$ acroleine beschermt tegen blootstelling aan $100 \mu \mathrm{M}$ acroleine 4 uur later. A549 cellen zijn echter veel beter beschermd tegen de toxiciteit geïnduceerd door acroleine dan BEAS-2B cellen. Dit verschil in gevoeligheid van de verschillende celtypes is al eerder gezien voor andere stoffen zoals multi-walled carbon nanotubes (MWCNTs) (8). A549 cellen blijken een hogere activatie van Nrf2-gemedieerde antioxidant systemen te hebben dan BEAS-2B cellen. In A549 cellen leidt een relatief lage concentratie aan acroleine tot een toename in GSH levels, meer Nrf2 nucleaire translocatie en een toename in expressie van YGCS and heme oxygenase-1 (HO-1), twee endogene antioxidant genen. De AgNPs hadden geen effect op GSH levels en gen expressie van $\gamma$ GCS. De aanwezigheid van Nrf2 in de nucleus en HO-1 gene expressie waren wel toegenomen. Voorbehandeling met een lage concentratie AgNPs, $5 \mu \mathrm{g} / \mathrm{cm}^{2}$ AgNP1 of $5 \mu \mathrm{g} / \mathrm{cm}^{2}$ AgNP2, beschermde ook tegen blootstelling aan een hoge concentratie acroleine van $100 \mu \mathrm{M}$. Dit geeft aan dat AgNPs een adaptieve respons induceren die niet alleen beschermt tegen de stof zelf, maar ook tegen andere stoffen, in dit geval acroleine, waar aannemelijk een vergelijkbaar adaptief beschermingsmechanisme aan ten grondslag ligt. Dit laatste, dat blootstelling aan een lage concentratie van de ene stof beschermt tegen een hoge concentratie van een andere stof, hebben wij gedefinieërd als transhormesis. Het mechanisme hoe AgNPs tot toxiciteit kunnen leiden is nog steeds niet volledig bekend. Sommige studies laten zien dat AgNPs de vorming van reactieve zuurstof deeltjes (ROS) kunnen induceren (9).

\subsection{Antioxidant flavonoiden, bescherming en het mechanisme van adaptatie}

Flavonoiden zijn polyfenolen die aanwezig zijn in groente en fruit. In deze thesis, zijn de meest voorkomende flavonoiden rutine en quercetine bestudeerd. Ze kunnen ROS wegvangen en fungeren hierbij als antioxidant. Door het wegvangen van ROS worden ze omgezet in hun geoxideerde vorm, het zogenaamde flavonoid quinone. Hierdoor beschermen ze tegen de schadelijke effecten van ROS. Als de ROS niet worden 
weggevangen, leidt dit tot schade aan deoxyribonucleïnezuur (DNA), proteïnen of lipiden (10). De quinone vorm van het flavonoid is thiol reactief. Afhankelijk van de structuur reageert het quinone hetzij met GSH of vitamine $\mathrm{C}$ binnen het endogeen antioxidant netwerk (11). Door de rutinose groep, is rutine een veel hardere electrofiel dan quercetine. Daardoor zal rutine veel liever met vitamine $\mathrm{C}$ reageren dan met GSH, omdat vitamine $\mathrm{C}$ ook een veel hardere nucleofiel is. Harde electrofielen reageren namelijk liever met harde nucleofielen en zachte electrofielen met zachte nucleofielen volgens het Hard Zacht Zuur Base (HSAB) concept. GSH en vitamine $\mathrm{C}$ zijn niet de enige endogene antioxidanten. In deze thesis, in Hoofdstuk 5, hebben we de antioxidant werking van rutine bevestigd, omdat deze tot minder endogene oxidatieve stress leidt. Het gevormde rutine quinone kan vervolgens met thiolen in Keap1 reageren en de activiteit van het endogene antioxidant enzym thioredoxin reductase 1 (TrxR1) verminderen. Dit zal vervolgens tot een toename van de Nrf2 activiteit leiden. Ook werd een stijging gemeten in de gen expressie van glutamaat cysteine ligase (GCL) in de humane navelstreng ader endotheel cellen (HUVECs). Verder, liet oxidatieve stress de proinflammatoire activiteit van nuclear factor kappa-light-chain-enhancer of activated B cells (NF-KB) en pro-angiogenetische activiteit van hypoxia induceerbare factor (HIF) toenemen. Rutine quinone kon de inductie van $\mathrm{Nf}$ $\mathrm{KB}$ en HIF voorkomen. Eerder is aangetoond dat een verhoogd GCL level leidt tot een toename in GSH hoeveelheden. Dit kan de adaptieve respons als gevolg van rutine quinone verklaren. Bovendien is deze respons doelgericht, omdat deze alleen plaatsvindt wanneer er oxidatieve stress is. Wanneer cellen worden blootgesteld aan rutine quinone en 3 uur later aan oxidatieve stress, wordt er minder oxidatieve stress gemeten dan wanneer de cellen niet zijn voorbehandeld met rutine quinone, maar alleen zijn blootgesteld aan oxidatieve stress. Het fenomeen dat een algemeen aanvaarde niet schadelijke stof een adaptieve respons induceert die beschermt tegen een toxische stof, in dit geval beschermt rutine quinone tegen oxidatieve stress, wordt parahormesis genoemd $(12,13)$. Om de fysiologische relevantie van dit fenomeen aan te tonen, is het effect van rutine op de vasomotor respons in bloedvaten onderzocht. Het is bevestigd dat oxidatieve stress de vasorelaxatie van arteriolen verminderd (14). Voorbehandeling van humane arteriolen van de placenta met rutine voorkwam de verminderde vasorelaxatie door oxidatieve stress. Dit is ten eerste te wijten aan de directe antioxidant activiteit van rutine. Deze directe antioxidant activiteit kan echter niet de langdurige beschermende werking van rutine in cardiovasculaire ziektes (CVD) verklaren, omdat rutine maar een korte halfwaardetijd heeft. Ten tweede, induceert rutine quinone protectie op langere termijn door de transcriptie van Nrf2-gemedieerde endogene antioxidanten te verhogen. Verder wordt de vasomotor respons van de vaten bevorderd door te supplementeren met thiolen hetgeen stikstofoxide (NO) productie promoot. Bovendien, is al eerder aangetoond dat de combinatie van rutine en waterstofperoxide $\left(\mathrm{H}_{2} \mathrm{O}_{2}\right)$ endotheel stikstofoxide synthase (eNOS) messenger ribonucleïnezuur (mRNA) laat toenemen (15). Dit effect is 
waarschijnlijk te wijten aan rutine quinone en niet aan rutine en resulteert uiteindelijk in meer vasorelaxatie van bloed vaten.

\subsection{Thiol reactiviteit en adaptatie versus amino reactiviteit en toxiciteit}

Acroleine, AgNPs en rutine quinone kunnen adaptatie induceren bij lage concentraties en kortdurende blootstelling. Voor acroleine hebben we ook gekeken naar de effecten van een hoge concentratie acroleine of een lange incubatietijd (Hoofdstuk 6). Bij blootstelling aan een lage concentratie acroleine of bij een korte incubatietijd, beschermt GSH tegen de schade geïnduceerd door acroleine. Wanneer de concentratie verhoogd wordt, of de incubatietijd toeneemt, zal acroleine het microsomale glutathion-Stransferase 1 (MGST1) activeren. Acroleine reageert dan via 1,4 Michaël additie met het thiol in cysteïne 49 van MGST1. Maar wanneer de concentratie van acroleine te hoog is of de incubatietijd te lang, zal acroleine naast de thiolen ook met de amino groepen van MGST1 reageren. Er wordt dan een Schiff base of thiazolidine derivaat gevormd, dat leidt tot remming van het MGST1 enzym. Dit geeft opnieuw aan dat GSH de eerste verdediging is tegen acroleine. Door MGST1 te activeren wordt een adaptieve respons geïnduceerd met effecten op korte termijn. Maar wanneer de concentratie van acroleine te hoog is of de incubatietijd te lang, dan raken de cellulaire beschermingssystemen verzadigd en zal acroleine met de vitale onderdelen van de cel reageren zoals proteïnen of DNA. Dit zal tot schade leiden en bijdragen aan de ontwikkeling van oxidatieve stress-gerelateerde ziekten. Om de ontwikkeling van deze ziektes te voorkomen, is het waarschijnlijk voordeliger voor het organisme om de endogene bescherming door MGST1 te verminderen en de activiteit van het enzym te verlagen door een reactie met de amino groepen van het enzym te stimuleren en celdood te bevorderen. Dit laatste mechanisme zal verder worden uitgelegd in de volgende paragraaf.

\subsection{Apoptose, een mechanisme van toxiciteit of bescherming?}

Naast rutine quinone geeft ook quercetine quinone adaptatie door Nrf2 activiteit te bevorderen. Daarnaast, kan quercetine quinone NF-KB en HIF activiteit geïnduceerd door oxidatieve stress ook verminderen.

In tegenstelling met normale cellen, geeft quercetine quinone in tumorcellen, in dit geval GSH-gedepleteerde A375 melanoma cellen, een afname in plaats van een toename in expressie van Nrf2-gemedieerde endogene antioxidant genen (Hoofdstuk 7). Ook vermindert quercetine quinone de activiteit van TrxR1. Tegelijkertijd, leidt blootstelling aan quercetin quinone tot meer apoptose, terwijl oxidatieve stress necrose induceert. Ook induceert quercetin quinone een toegenomen hoeveelheid glyceraldehyde-3-fosfaat dehydrogenase (GAPDH) in de kern dat gepaard gaat met 244 
toenemende trend in de ratio van expressie van pro- ten opzichte van anti-apoptotische genen (Hoofdstuk 7). A375 melanoma cellen die tegelijkertijd blootgesteld zijn aan oxidatieve stress en quercetine laten een remming van de Nrf2-gemedieerde adaptatie zien en een verschuiving in celdood van oxidatieve stress-geïnduceerde necrose naar apoptose. Dit onderbouwt dat quercetine in een cel die blootgesteld is aan veel oxidatieve stress, een tumor cel, in plaats van de cel te beschermen tegen schade aan essentiële proteïnen of DNA, geprogrammeerde celdood of apoptose induceert. Dit is natuurlijk toxisch voor de cel, maar beschermt het organisme tegen de schadelijke effecten van beschadigde, niet goed functionerende cellen. Dit is een doelgericht effect van quercetine, omdat het alleen gebeurt wanneer er veel oxidatieve stress is bijvoorbeeld in tumorcellen waar onder andere ook meer Nrf2, TrxR1 en GAPDH is dan in normale cellen.

\section{Implicaties, limitaties en aanbevelingen voor toekomstig onderzoek}

Meer kennis van onze mogelijkheid om ons aan te passen en de rol van de tijd hierin, zal ons begrip vergroten hoe organismen hun redox signalering aan kunnen passen. Door deze kennis zouden we kunnen interfereren en sturen in dit proces om op het juiste moment adaptatie, de mogelijkheden van het organisme om zich aan te passen aan de veranderingen in de omgeving, te bevorderen. Hoe beter een organisme beschermd is tegen oxidatieve stress, des te minder kans is er dat dit de interne redox balans verstoort en zich oxidatieve stress-gerelateerde ziekten ontwikkelen zoals chronic obstructive pulmonary disease (COPD), CVD of kanker. In deze thesis hebben we de huidige kennis van tijd in adaptatie uitgewerkt. Metname met betrekking tot adaptatie op de lange termijn is nog niet alles bekend en kan nog veel onderzocht worden. Bijvoorbeeld kunnen we onbeperkt adaptatie induceren of raken we gewend aan een bepaalde blootstelling en verdwijnt onze mogelijkheid om ons aan te passen op termijn? Vindt er desensitizatie plaats? Het mechanism van adaptatie is vergelijkbaar voor sommige chemicaliën zoals acroleine en AgNPs. Maar welke chemicaliën kunnen een adaptieve respons induceren die ook beschermt tegen een andere stof? Is dit voor alle stoffen hetzelfde? Of zijn er ook verschillende mechanismen van adaptatie die door verschillende stoffen worden geïnduceerd en kunnen deze stoffen dan nog steeds transhormesis induceren? Om deze vragen te beantwoorden is er verder onderzoek nodig. Bovendien is het concept van hormesis op dit moment nog niet opgenomen in de wetgeving over risico evaluatie van toxische stoffen. Moeten we onze drempel limiet waarde (THV) of acceptabele dagelijkse inname (ADI) voor stoffen verhogen om er zeker van te zijn dat adaptatie wordt geïnduceerd die vervolgens beschermt tegen toekomstige blootstellingen. De grootste beperking van adaptatie is dat het energie kost, die verspild is wanneer we in de toekomst 
niet worden blootgesteld. En of we in de toekomst worden blootgesteld aan een toxische stof kunnen we ook niet altijd voorspellen.

Deze thesis heeft aangetoond dat het mechanisme dat adaptatie veroorzaakt vergelijkbaar is voor acroleine en AgNPs. Echter welk mechanisme hier precies aan ten grondslag ligt, is nog onduidelijk. Wel is zowel voor acroleine als AgNPs gevonden dat Nrf2 hierbij betrokken is. Om te bevestigen dat Nrf2 de adaptatie geïnduceerd door acroleine of AgNPs veroorzaakt, zou een Nrf2 knock out studie moeten worden uitgevoerd. De bevinding dat verschillende stoffen verschillende Nrf2 gereguleerde genen activeren, kan worden verklaard doordat er waarschijnlijk verschillende transcriptiefactoren betrokken zijn bij de regulatie van een bepaald gen. Verder kunnen epigenetische processen een rol spelen en is de tijd van belang. Immers, GSH schijnt belangrijk te zijn in de korte termijn bescherming tegen acroleine, terwijl op langere termijn HO-1 expressie wordt geïnduceerd. Verder is het aanbevolen om het principe van hormesis en transhormesis ook voor andere nanodeeltjes (NPs) zoals titanium dioxide $\left(\mathrm{TiO}_{2}\right)$ of goud nanodeeltjes (AuNPs) te verifiëren.

Rutine quinone kan ook adaptatie induceren door de expressie van Nrf2gemedieerde endogene antioxidanten te stimuleren. De effecten van rutine quinone zijn echter alleen indirect aangetoond. Om een oorzakelijk effect van Nrf2 in de rutine quinone geïnduceerde adaptatie te kunnen bevestigen, is verder onderzoek met een Nrf2 knock out model noodzakelijk. Verder is het aan te raden de rutine quinone vorming en adduct vorming met specifieke proteïnen zoals Keap1 intracellulair aan te tonen. Toekomstige experimenten moeten rekening houden met het lage expressie level van Keap1 en niet-reducerende gels zouden toegepast moeten worden om te voorkomen dat het adduct tijdens het experiment verbroken wordt. Toekomstig onderzoek zou zich ook kunnen richten op het aantonen van adaptatie in bloedvaten, door ze voor te behandelen met rutine quinone en een paar uur later bloot te stellen aan oxidatieve stress, waarna de effecten op de vasomotor respons gemeten kunnen worden. Tot slot, zou het erg interessant zijn om het gevonden effect voor rutine quinone uit te breiden door de endotheel monolayer integriteit (of vasculaire lekkage) te meten met behulp van elektrisch cel-substraat bioimpedantie detectie (ECIS) (16). Hiermee kunnen de redoxgeïnduceerde vasculaire effecten gekoppeld worden aan inflammatie. Een kanttekening hierbij is dat rutine quinone waarschijnlijk veel selectiever met bepaalde cellulaire proteïnen reageert dan ROS. Een van deze proteïnen zou Keap1 kunnen zijn. Keap1 functioneert als sensor voor bepaalde stoffen. Zo reageert acroleine net als andere alkenal-achtige stoffen zoals 4-hydroxy-2-nonenal, met cysteïne 288 van Keap1, terwijl rutine quinone met cysteïne 151 reageert (6). Cysteïne 288 bevindt zich in de zogenaamde 'intervening region' en cysteïne 151 in het 'Bric-à-Brac (BTB)' domein van Keap1. Binding 
aan cysteïne 288 zal tot een conformationele verandering leiden, terwijl binding aan cysteïne 151, interfereert met de dimeer vorming van Keap1 en binding aan het cytoplasmatische cytoskelet (17). In beide gevallen, zal de interactie van Keap1 met Nrf2 worden aangetast, hetgeen leidt tot een activatie van endogene antioxidant genen downstream. De verzameling aan cysteïnes in Keap1 die reageren, is afhankelijk van de stof. Naast de effecten gerelateerd aan Keap1, zouden ook de GSH levels in de tijd gemeten kunnen worden en is het van belang de invloed hiervan op NO productie en daarmee geassocieerde vasorelaxatie te analyseren. Verder zou het nieuwe mechanisme dat de langdurige bescherming door rutine verklaart, ook in de toekomst kunnen bijdragen aan een beter begrip hoe rutine het risico op het ontwikkelen van CVD kan verminderen. Tot slot zou ook getest kunnen worden of andere flavonoiden met een klein verschil in chemische structuur, adaptatie kunnen induceren. Wellicht zal een verschil in hardheid van het flavonoid molecuul, bepalen hoe potent de adaptatie is die wordt geïnduceerd en hoe stabiel de adducten zijn met Keap1.

Met betrekking tot het onderzoek van acroleine en MGST1, zou kunnen worden uitgezocht of de binding van acroleine aan amino groepen van MGST1, de enige oorzaak is van de remming van het enzym. Om dit te verifiëren zouden de amino groepen chemisch afgeschermd kunnen worden, alvorens MGST1 bloot te stellen aan hoge concentraties van acroleine of lange incubatietijden met acroleine. Tot slot, zou de specifieke amino groepgemedieerde remming van MGST1 gevalideerd moeten worden in tumor cellen. Zo zou een mogelijke rol van de aminogroepen als aangrijpingspunt om de resistentie tegen antikanker geneesmiddelen tegen te gaan kunnen worden geverifieërd. Waarschijnlijk is acroleine in dit geval geen geschikte stof, maar flavonoid quinonen zouden overwogen kunnen worden. Wanneer een flavonoid, die ook aanwezig in onze dagelijkse voeding, als therapie wordt gebruikt, wordt deze ook wel gedefinieërd als nutraceutical.

Of de gemeten apoptose geïduceerd door quercetine quinone een gevolg is van de GAPDH translocatie naar de nucleus, kan worden bevestigd met een (tijdelijk) knock out model voor GAPDH in de kern. Een tweede suggestie voor toekomstig onderzoek is om ook het GAPDH-quercetine adduct in de cel te laten zien.

Ten slotte, zijn er indicaties dat adaptatie ook in vivo plaatsvindt. Zo hebben chronische rokers bijvoorbeeld meer GSH in hun long epitheelvloeistof dan niet-rokers of acute rokers (18). Alhoewel, in dit geval moet nog bevestigd worden of blootstelling aan een lage dosis van een bepaalde stof op termijn beschermt tegen blootstelling aan een hoge dosis van dezelfde of een andere stof met hetzelfde adaptatie mechanisme. Toch zal de risico evaluatie van toxische stoffen zeker veranderen als gevolg van deze bevinding. De maatschappelijk gevolgen zullen ongetwijfeld ethische vraagstukken oproepen. 
Het Janus gezicht van Paracelsus
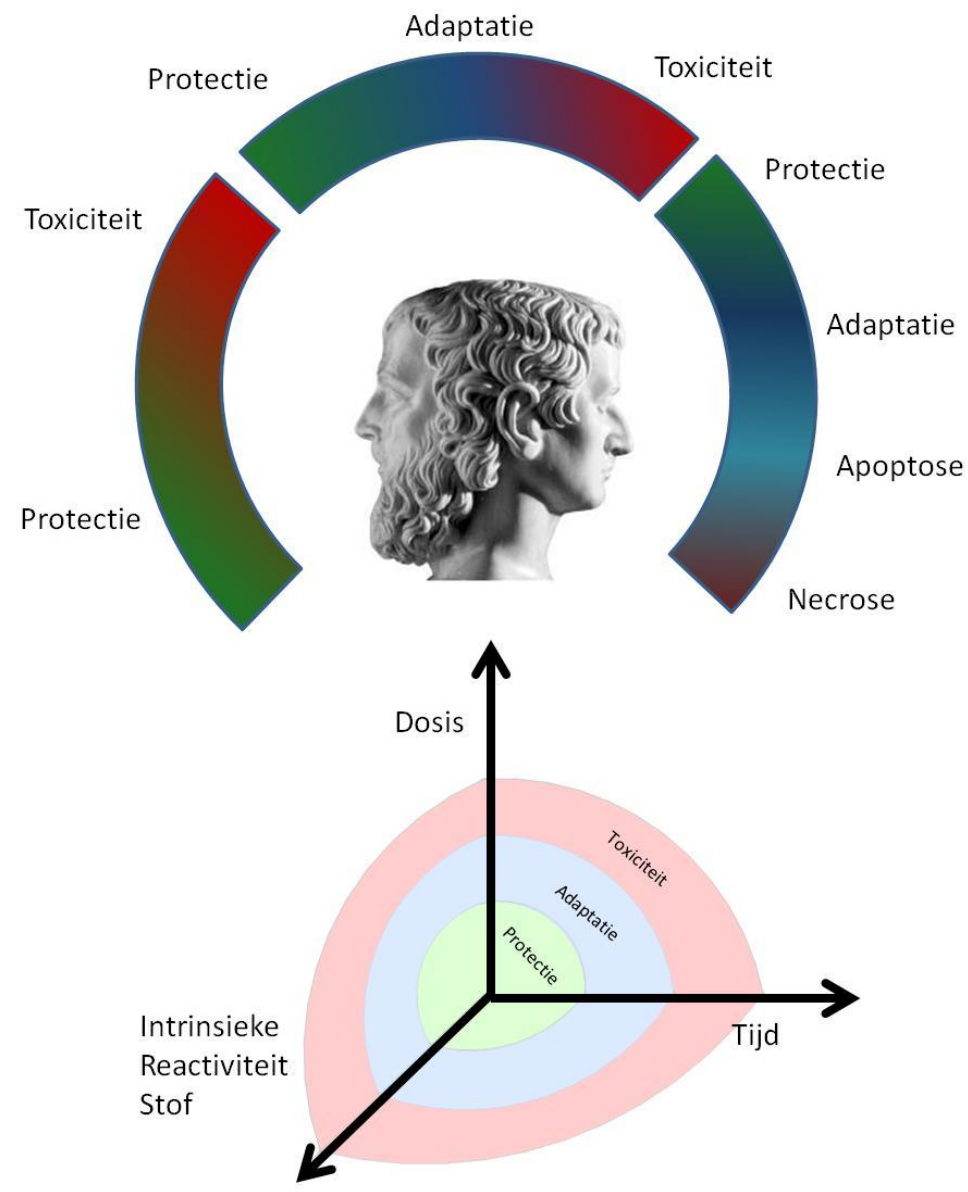

Figuur 1: Paracelsus vanuit Janus bekeken: het paradigma van Paracelsus, "Sola dosis facit venenum", heeft zich in de loop der tijd ontwikkeld rekening houdend met de dosis, tijd en intrinsieke activiteit van de desbetreffende stof.

\section{Conclusie}

Het paradigm van Paracelsus heeft zich in de loop der tijd ontwikkeld (Figuur 1). Terugkijkend stelde hij in 1500: 'Sola dosis facit venenum' (in het Nederlands: De dosis maakt het gif), dat betekent dat iedere stof uiteindelijk toxisch zal zijn, afhankelijk van de dosis die je toedient. Bij een lage dosis, kunnen de verdedigingssystemen in ons lichaam bescherming bieden zoals het GSH en Thioredoxin (Trx) redox systeem. Bij een te hoge 
dosis, raken deze beschermingssystemen verzadigd en kan een stof toxische effecten induceren. In deze thesis, hebben wij het paradigma van Paracelsus verfijnd door een extra dimensie aan te brengen in de range tussen protectie en toxiciteit: namelijk adaptatie. Wanneer een stof net niet toxisch is, en de protectie systemen niet volledig kunnen beschermen, zal deze stof selectief specifieke pathways induceren die bijdragen tot adaptatie en bescherming op de langere termijn, bijvoorbeeld een toename in transcriptie van endogene antioxidant enzymen. Dit is het huidige paradigma, dat verder geillustreerd is in deze thesis. Dit paradigma blijft echter, net als het leven op aarde, voortdurend aan verandering onderhevig en zal zich in de toekomst verder ontwikkelen. Zo zullen er naar verwachting in de toekomst additionele niveaus en sub niveaus worden toegevoegd. Toxiciteit kan al worden onderverdeeld in apoptose en necrose. Sommige stoffen stimuleren apoptose, zoals quercetine quinone, terwijl andere necrose bevorderen, bijvoorbeeld $\mathrm{H}_{2} \mathrm{O}_{2}$ dat oxidatieve stress induceert. Hiernaast kan er nog een andere dimensie worden toegevoegd, namelijk de dimensie van de intrinsieke reactiviteit van bepaalde stoffen of de aard van de stof. Een van de strategieën binnen de toxicologie is om te beschermen tegen de reactiviteit van een bepaalde stof door deze te neutralizeren. Zo werd een antioxidant oorspronkelijk toegevoegd om de reactiviteit van ROS te verzwakken. In het huidige paradigma, wordt de reactiviteit selectief gestuurd. De reactiviteit van ROS wordt niet verzwakt, maar een antioxidant zoals quercetine wordt toegevoegd, om vervolgens door ROS te worden omgezet in quercetine quinone dat selectief adaptatie induceert door de expressie van endogene antioxidant systemen te verhogen, die vervolgens kunnen beschermen tegen een toekomstige blootstelling aan ROS. Afhankelijk van de omstandigheden, kan adaptatie gunstig of schadelijk zijn. Het versterken van de endogene antioxidant respons in tumorcellen is een niet gewenste adaptatie, terwijl toxiciteit, in het bijzonder apoptose, voordelig kan zijn in een kanker cel. Echter, hoe dit paradigma zich in de toekomst zal ontwikkelen en wat we kunnen doen om dit proces te sturen, zal de toekomst uitwijzen. Tempus omnia revelat. 


\section{Referenties}

1. Davies KJ. Adaptive homeostasis. Mol Aspects Med. 2016 Jun;49:1-7.

2. Mattson MP. Hormesis defined. Ageing research reviews. 2008 Jan;7(1):1-

7.

3. Sthijns MM, Weseler AR, Bast A, Haenen GR. Time in Redox Adaptation Processes: From Evolution to Hormesis. International journal of molecular sciences. 2016 Sep 29;17(10).

4. Sthijns MM, Thongkam W, Albrecht C, Hellack B, Bast A, Haenen GR, et al. Silver nanoparticles induce hormesis in A549 human epithelial cells. Toxicology in vitro : an international journal published in association with BIBRA. 2017 Jan 18.

5. Sthijns MM, Randall MJ, Bast A, Haenen GR. Adaptation to acrolein through upregulating the protection by glutathione in human bronchial epithelial cells: the materialization of the hormesis concept. Biochemical and biophysical research communications. 2014 Apr 18;446(4):1029-34.

6. McMahon M, Lamont DJ, Beattie KA, Hayes JD. Keap1 perceives stress via three sensors for the endogenous signaling molecules nitric oxide, zinc, and alkenals. Proc Natl Acad Sci U S A. 2010 Nov 02;107(44):18838-43.

7. Nassini R, Materazzi S, Andre E, Sartiani L, Aldini G, Trevisani M, et al. Acetaminophen, via its reactive metabolite $\mathrm{N}$-acetyl-p-benzo-quinoneimine and transient receptor potential ankyrin-1 stimulation, causes neurogenic inflammation in the airways and other tissues in rodents. FASEB journal : official publication of the Federation of American Societies for Experimental Biology. 2010 Dec;24(12):4904-16.

8. Ursini CL, Cavallo D, Fresegna AM, Ciervo A, Maiello R, Buresti G, et al. Differences in cytotoxic, genotoxic, and inflammatory response of bronchial and alveolar human lung epithelial cells to pristine and $\mathrm{COOH}$-functionalized multiwalled carbon nanotubes. Biomed Res Int. 2014;2014:359506.

9. Kermanizadeh A, Vranic S, Boland S, Moreau K, Baeza-Squiban A, Gaiser $B K$, et al. An in vitro assessment of panel of engineered nanomaterials using a human renal cell line: cytotoxicity, pro-inflammatory response, oxidative stress and genotoxicity. BMC Nephrol. 2013 Apr 25;14:96.

10. Tabak O, Gelisgen R, Erman H, Erdenen F, Muderrisoglu C, Aral H, et al. Oxidative lipid, protein, and DNA damage as oxidative stress markers in vascular complications of diabetes mellitus. Clin Invest Med. 2011 Jun 01;34(3):E163-71. 
11. Jacobs $H$, Moalin M, Bast A, van der Vijgh WJ, Haenen GR. An essential difference between the flavonoids monoHER and quercetin in their interplay with the endogenous antioxidant network. PLoS One. 2010 Nov 08;5(11):e13880.

12. Ursini F, Maiorino M, Forman HJ. Redox homeostasis: The Golden Mean of healthy living. Redox biology. 2016 Aug;8:205-15.

13. Forman HJ, Davies KJ, Ursini F. How do nutritional antioxidants really work: nucleophilic tone and para-hormesis versus free radical scavenging in vivo. Free radical biology \& medicine. 2014 Jan;66:24-35.

14. Thengchaisri N, Hein TW, Wang W, Xu X, Li Z, Fossum TW, et al. Upregulation of arginase by $\mathrm{H} 2 \mathrm{O} 2$ impairs endothelium-dependent nitric oxidemediated dilation of coronary arterioles. Arteriosclerosis, thrombosis, and vascular biology. 2006 Sep;26(9):2035-42.

15. Ugusman A, Zakaria Z, Chua KH, Nordin NA, Abdullah Mahdy Z. Role of rutin on nitric oxide synthesis in human umbilical vein endothelial cells. ScientificWorldJournal. 2014;2014:169370.

16. van Wetering S, van Buul JD, Quik S, Mul FP, Anthony EC, ten Klooster JP, et al. Reactive oxygen species mediate Rac-induced loss of cell-cell adhesion in primary human endothelial cells. J Cell Sci. 2002 May 01;115(Pt 9):1837-46.

17. Kansanen E, Kuosmanen SM, Leinonen H, Levonen AL. The Keap1-Nrf2 pathway: Mechanisms of activation and dysregulation in cancer. Redox biology. 2013 Jan 18;1:45-9.

18. Rahman I, MacNee W. Lung glutathione and oxidative stress: implications in cigarette smoke-induced airway disease. Am J Physiol. 1999 Dec;277(6 Pt 1):L1067-88. 



\section{Valorization addendum}




\section{Valorization addendum}

\subsection{Relevance}

In this thesis, the response of a cell to a toxic compound (toxin), namely protection, adaptation or cell death, was investigated. In particular, the cellular response to the compounds acrolein, silver nanoparticles (AgNPs) and the flavonoids rutin and quercetin was examined. In the cellular response to these substances, redox (regulated) mechanisms via the induction of oxidative stress play a pivotal role.

Oxidative stress does not only play a role in the cellular toxicity of these compounds, it also plays a pivotal role in various age-related diseases including chronic obstructive pulmonary disease (COPD), cardiovascular disease (CVD) and cancer.

\subsubsection{Chronic Obstructive Pulmonary Disease}

Worldwide 65 million people have COPD. And every year, 3 out of 1000 are diagnosed with COPD $(1,2)$. It is a severe disease and current treatment is mostly aimed at preventing disease progression and reducing the symptoms, since a cure has not been found. Approximately $5 \%$ of all deaths are due to COPD. This makes COPD the fifth leading cause of death worldwide and it has been predicted that in 2030, it will even be the third leading cause of death. Especially, smoking is involved in causing COPD. The prevalence of COPD in smokers is 3 times higher than in non-smokers (3). Next to smoking also other factors like age and gender are risk factors for developing COPD.

\subsubsection{Cardiovascular disease}

CVD results in almost 20 million deaths globally every year (4-6). This makes it the primary cause of death worldwide. The mortality risk is expected to increase by $36 \%$ from 2017 to 2030. CVD is not one disease, but an umbrella term for a collection of diseases including cardiomyopathy, heart failure, coronary artery disease and cerebrovascular disease including stroke. Once developed stroke, the risk of reoccurrence is approximately $30 \%$. Many risk factors are involved in the development of CVD including age, gender, high blood pressure or physical inactivity. Remarkably, in $50 \%$ of the strokes, hypertension plays a central role (7). The resulting hospitalization is the main contributing factor to the enormous health care costs of approximately 313 billion euro worldwide annually. The prevalence of hypertension is about 1000 million people. The prevalence of hypertension increases with age. Additionally, almost the whole population is at risk of developing CVD, since according to the Framingham Heart Study, $90 \%$ of all the people will develop hypertension (8). Additionally, current diagnostic and treatment methods are lacking. Diagnosis is mainly focused on late stage risk factors and treatment is symptomatic rather than curative or preventive. 


\subsubsection{Cancer}

Worldwide 25 million people have cancer $(9,10)$. And every year it is estimated that 10 million people are newly diagnosed with cancer. And this number is even increasing to 12 million new cases each year by 2030 . The associated mortality is also very high, namely around 8 million deaths globally each year. This makes cancer the second leading cause of death worldwide. Cancer is a very heterogenous disease and can affect nearly any organ, most deaths are attributed to lung, liver, colorectal, stomach and breast cancer. The treatment is not very specific and is most successful when applied as early as possible. Unfortunately, most cancer types are diagnosed at late stage and screening methods still need to be improved. It is estimated that only $30-50 \%$ of the cancers currently are preventable and the health care costs amount almost 1 trillion euro annually.

This extensive burden of the oxidative stress-related diseases including COPD, CVD and cancer underlines the need to develop strategies to treat patients suffering from the disease or prevent development or progression of the pathologic process (11-14). These diseases are complex and different factors are involved, but the common characteristic is the pivotal role of oxidative stress in their etiology. Acrolein induces oxidative stress and is mainly associated with an increase in the prevalence of COPD (1517). The toxic effects of AgNPs are still enigmatic, although induction of oxidative stress seems to be involved (18-22). Evidence is emerging that the flavonoids, rutin and quercetin can protect against oxidative stress-related diseases, because of their antioxidant function (23). They can scavenge reactive oxygen species (ROS) and thereby protect against oxidative stress-related damaging effects to protein, lipid or DNA (24). Epidemiological studies regarding the expected beneficial effect of rutin or quercetin in the treatment or prevention of CVD or cancer are however controversial (25-31). In this thesis it was therefore further investigated via which mechanism these toxins induce their effects. Apparently, besides inducing protection (rutin, quercetin) or cell death (acrolein, AgNPs), all of these substances have also shown to induce adaptation (32-34). This implies that exposure to a low dose of the chemical for a relative short time is not toxic and can upregulate endogenous antioxidant systems that protect against a second challenge with a toxic, relative high dose of the specific toxin or exposure to the toxin for a relatively long time (35).

Risk assessment of toxins (36) starts with hazard identification and exposure assessment and in the European Union this is organized by REACH (Registration, Evaluation, Authorization and Restriction of Chemicals) (37) and OECD (Organization for Economic Co-operation and Development) that also makes rules and regulations based on this risk characterization. Some chemicals have a threshold and others no threshold for their (toxic) effect. For some substances, even zero exposure is recommended. Current risk assessment procedures do not include the phenomenon of adaptation. Risk 
assessment of especially acrolein and AgNPs with a non-observed adverse effect level (NOAEL) of $0.1 \mu \mathrm{g} / \mathrm{m}^{3}$ and $100 \mu \mathrm{g} / \mathrm{m}^{3}$ respectively should be reconsidered $(38,39$ ).

Regarding rutin and quercetin, a better understanding of their molecular mechanism inducing adaptation or toxicity could result in a more targeted use in the treatment of CVD or cancer. The results in this thesis indicate that, after in vivo validation, rutin could may contribute to the therapy against CVD. Rutin is present in the daily human diet, mainly in fruit and vegetables like citrus fruits and buckwheat, it is a food component. If rutin is going to be used as a drug against hypertension and concomitant CVD, it will be a food component or nutrient that is used as a pharmaceutical drug, which can be defined as a nutraceutical. The most striking difference compared to a pharmaceutical, is that a nutraceutical mostly has multiple targets and effects (40).

Rutin in the presence of oxidative stress has been shown to upregulate Nrf2 and associated antioxidant genes, which could function as an early biomarker of CVD, which is likely better than the many current circulating biomarkers like C-reactive protein or triglycerides or a decreased high density lipoprotein. In the end it may lead to an aggressive decrease of atherosclerosis modifiers (ADAM) or development of new types of interventions and prevent early vascular aging (EVA) (41).

With regard to quercetin it is remarkable that in BSO-treated A375 tumor cells in regions with high oxidative stress Nrf2-mediated expression of endogenous antioxidant genes is decreased, while apoptosis is induced, whereas in (surrounding) non-tumor

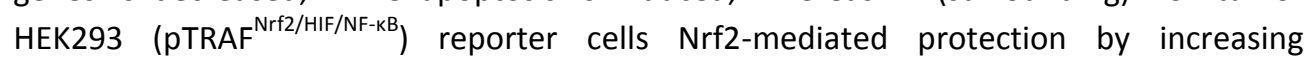
endogenous antioxidant systems is enhanced. This indicates that the effect of the flavonoid is dependent on the presence of oxidative stress. To be able to use quercetin in current clinical practice as a treatment against cancer, more research is needed. The findings should be verified in vivo. The effects of quercetin will be dependent on the circumstances, level of oxidative stress and endogenous antioxidant presence, pointing at a very personalized potential regarding treatment or prevention of cancer by personalized nutrition.

\subsection{Target groups}

The findings from this thesis after verification in vivo could contribute to preventing or treating oxidative stress-related diseases like COPD, CVD or cancer. The knowledge obtained could also be expanded to the prevention or treatment of other oxidative stress-related diseases, for example diabetes. By this way it could be aimed to decrease the disease burden, to improve the life of the concerned patients and to diminish the related health care costs also resulting in some economical benefits. Furthermore, rules and regulations especially regarding risk assessment of the investigated toxins should be reconsidered on a national level, by the 'Rijksinstituut voor 
Volksgezondheid en Milieu' (RIVM) or international level for example by the REACH or OECD from the European Union or the World Health Organization (WHO).

\subsection{Activities and products}

In particular, with regard to the antioxidant flavonoids, the chemical structure can be modified to either improve the uptake and bioavailability or activity of the specific flavonoid $(42,43)$. By this way a better therapeutic agent can be achieved with higher reactivity or better metabolic profile. Pharmaceutical companies and food industries will be interested, since on this modified flavonoid a patent can be requested and this nutrient can be developed to a new pharmaceutical treatment, a nutraceutical, which can be launched. This treatment can be personalized, since each patient may respond differently and the flavonoid could be changed in structure accordingly.

\subsection{Planning and realization}

Before a modified flavonoid can be launched as a new pharmaceutical product on the market or Nrf2 can be used as a biomarker, further studies are required. First, the found effects need to be validated further in in vitro and in vivo studies. Additionally, before the modified flavonoid can be commercialized, it needs to be validated in Phase IIV trials, which will take a couple of years. Besides this pharmaceutical approach it might also be considered to adjust the current human diet. This can be done in a personalized way, resulting in personalized nutrition. In some cases the use of supplements of flavonoids could be recommended. Finally, it is aimed to change the current treatment recommendations in the European medicine evaluation board (MEB) or the 'Nederlands huisartsen genootschap' (NHG) standard for the investigated oxidative stress-related diseases including COPD, CVD and cancer. Regarding, the risk assessment of the toxins acrolein and AgNPs, NOAEL can be changed relatively fast by the REACH, OECD or the RIVM based on the current study of adaptive effects of the concerned chemicals and future supportive additional findings.

\subsection{Innovation}

In this thesis it has been described that the original view of Paracelsus on protection and toxicity should be extended by incorporating the level of adaptation or hormesis. A subtoxic dose of a chemical selectively targets specific pathways that induce adaptation and contributes to an enhanced protection on a longer term. It is found that this principle applies for the toxins acrolein, AgNPs and the flavonoids rutin and quercetin in the presence of oxidative stress. Additionally, we introduce a new term, the phenomenon of transhormesis, indicating that a low dose of one chemical induces a protective system (adaptation) that protects against the toxic dose of another chemical 
for which a similar protective mechanism is induced by the adaptive response. Finally, the flavonoids rutin and quercetin only induce adaptation in the regions with a high level of oxidative stress. This makes the process selective and explains that despite of their short half-life, these flavonoids can be beneficial on a longer term. Apart from inducing adaptation, we also found that under specific circumstances flavonoids also enhance apoptotic cell death. This could explain the controversial epidemiologic findings regarding flavonoid intake in patients suffering from oxidative stress-related diseases. Furthermore, this adds another dimension to the paradigm of Paracelsus. The induction of adaptation or toxicity could both be beneficial or detrimental depending on the circumstances. For a healthy cell protective adaptation is beneficial, whereas in a tumor cell apoptotic cell death could be advantageous. This finding opens new directions to develop a personalized treatment or nutrition with flavonoids. In addition, the increased level of Nrf2 could be developed into a new biomarker that inversely predicts the progression of CVD. It could be an earlier biomarker than for example hypertension and most current tissue biomarkers. But in these promising innovative findings time plays an important role and it will also take some time before they are implemented in current clinical practice. 


\section{References}

1. Rycroft CE, Heyes A, Lanza L, Becker K. Epidemiology of chronic obstructive pulmonary disease: a literature review. International journal of chronic obstructive pulmonary disease. 2012;7:457-94.

2. ; Available from: http://www.who.int/respiratory/copd/burden/en/.

3. Raherison C, Girodet PO. Epidemiology of COPD. European respiratory review : an official journal of the European Respiratory Society. 2009 Dec;18(114):213-21.

4. Writing Group M, Mozaffarian D, Benjamin EJ, Go AS, Arnett DK, Blaha MJ, et al. Executive Summary: Heart Disease and Stroke Statistics--2016 Update: A Report From the American Heart Association. Circulation. 2016 Jan 26;133(4):447-54.

5. Cardiovascular disease in Europe 2016: an epidemiological update. European heart journal. 2016 Nov 07;37(42):3182-3.

6. Townsend N, Wilson L, Bhatnagar P, Wickramasinghe K, Rayner M, Nichols M. Cardiovascular disease in Europe: epidemiological update 2016. European heart journal. 2016 Nov 07;37(42):3232-45.

7. Sowers JR, Epstein M, Frohlich ED. Diabetes, hypertension, and cardiovascular disease: an update. Hypertension. 2001 Apr;37(4):1053-9.

8. Vasan RS, Beiser A, Seshadri S, Larson MG, Kannel WB, D'Agostino RB, et al. Residual lifetime risk for developing hypertension in middle-aged women and men: The Framingham Heart Study. Jama. 2002 Feb 27;287(8):1003-10.

9. Kamangar F, Dores GM, Anderson WF. Patterns of cancer incidence, mortality, and prevalence across five continents: defining priorities to reduce cancer disparities in different geographic regions of the world. Journal of clinical oncology : official journal of the American Society of Clinical Oncology. 2006 May 10;24(14):2137-50.

10. Jemal A, Center MM, DeSantis C, Ward EM. Global patterns of cancer incidence and mortality rates and trends. Cancer epidemiology, biomarkers \& prevention : a publication of the American Association for Cancer Research, cosponsored by the American Society of Preventive Oncology. 2010 Aug;19(8):1893-907.

11. Repine JE, Bast A, Lankhorst I. Oxidative stress in chronic obstructive pulmonary disease. Oxidative Stress Study Group. Am J Respir Crit Care Med. 1997 Aug;156(2 Pt 1):341-57.

12. Madamanchi NR, Vendrov A, Runge MS. Oxidative stress and vascular disease. Arteriosclerosis, thrombosis, and vascular biology. 2005 Jan;25(1):29-38.

13. Rahman T, Hosen I, Islam MMT, Shekhar HU. Oxidative stress and human health. Advances in Bioscience and Biotechnology. 2012;3:997-1019.

14. Reuter S, Gupta SC, Chaturvedi MM, Aggarwal BB. Oxidative stress, inflammation, and cancer: how are they linked? Free Radic Biol Med. 2010 Dec 01;49(11):1603-16. 
15. Costa DL, Kutzman RS, Lehmann JR, Drew RT. Altered lung function and structure in the rat after subchronic exposure to acrolein. The American review of respiratory disease. 1986 Feb;133(2):286-91.

16. Jakab GJ. Adverse effect of a cigarette smoke component, acrolein, on pulmonary antibacterial defenses and on viral-bacterial interactions in the lung. The American review of respiratory disease. 1977 Jan;115(1):33-8.

17. Mohammad MK, Avila D, Zhang J, Barve S, Arteel G, McClain C, et al. Acrolein cytotoxicity in hepatocytes involves endoplasmic reticulum stress, mitochondrial dysfunction and oxidative stress. Toxicology and applied pharmacology. 2012 Nov $15 ; 265(1): 73-82$.

18. Bohmert L, Niemann B, Lichtenstein D, Juling S, Lampen A. Molecular mechanism of silver nanoparticles in human intestinal cells. Nanotoxicology. 2015;9(7):852-60.

19. Foldbjerg R, Irving ES, Hayashi Y, Sutherland DS, Thorsen K, Autrup H, et al. Global gene expression profiling of human lung epithelial cells after exposure to nanosilver. Toxicol Sci. 2012 Nov;130(1):145-57.

20. Johnston HJ, Hutchison G, Christensen FM, Peters S, Hankin S, Stone V. A review of the in vivo and in vitro toxicity of silver and gold particulates: particle attributes and biological mechanisms responsible for the observed toxicity. Crit Rev Toxicol. 2010 Apr;40(4):328-46.

21. Liu W, Wu Y, Wang C, Li HC, Wang T, Liao CY, et al. Impact of silver nanoparticles on human cells: effect of particle size. Nanotoxicology. 2010 Sep;4(3):319-30.

22. Xia T, Kovochich M, Brant J, Hotze M, Sempf J, Oberley T, et al. Comparison of the abilities of ambient and manufactured nanoparticles to induce cellular toxicity according to an oxidative stress paradigm. Nano Lett. 2006 Aug;6(8):1794-807.

23. Haenen GR, Paquay JB, Korthouwer RE, Bast A. Peroxynitrite scavenging by flavonoids. Biochemical and biophysical research communications. 1997 Jul 30;236(3):5913.

24. Liu J, Wang X, Shigenaga MK, Yeo HC, Mori A, Ames BN. Immobilization stress causes oxidative damage to lipid, protein, and DNA in the brain of rats. FASEB journal : official publication of the Federation of American Societies for Experimental Biology. 1996 Nov;10(13):1532-8.

25. Haddad AQ, Venkateswaran V, Viswanathan L, Teahan SJ, Fleshner NE, Klotz LH. Novel antiproliferative flavonoids induce cell cycle arrest in human prostate cancer cell lines. Prostate cancer and prostatic diseases. 2006;9(1):68-76.

26. Cui L, Liu X, Tian Y, Xie C, Li Q, Cui H, et al. Flavonoids, Flavonoid Subclasses, and Esophageal Cancer Risk: A Meta-Analysis of Epidemiologic Studies. Nutrients. 2016 Jun $08 ; 8(6)$. 
27. Omenn GS, Goodman GE, Thornquist MD, Balmes J, Cullen MR, Glass A, et al. Effects of a combination of beta carotene and vitamin $A$ on lung cancer and cardiovascular disease. The New England journal of medicine. 1996 May 02;334(18):1150-5.

28. Bjelakovic G, Nikolova D, Gluud LL, Simonetti RG, Gluud C. Mortality in randomized trials of antioxidant supplements for primary and secondary prevention: systematic review and meta-analysis. Jama. 2007 Feb 28;297(8):842-57.

29. Suen J, Thomas J, Kranz A, Vun S, Miller M. Effect of Flavonoids on Oxidative Stress and Inflammation in Adults at Risk of Cardiovascular Disease: A Systematic Review. Healthcare. 2016 Sep 14;4(3).

30. Hooper L, Kroon PA, Rimm EB, Cohn JS, Harvey I, Le Cornu KA, et al. Flavonoids, flavonoid-rich foods, and cardiovascular risk: a meta-analysis of randomized controlled trials. Am J Clin Nutr. 2008 Jul;88(1):38-50.

31. Bazzano LA, He J, Ogden LG, Loria CM, Vupputuri S, Myers L, et al. Fruit and vegetable intake and risk of cardiovascular disease in US adults: the first National Health and Nutrition Examination Survey Epidemiologic Follow-up Study. Am J Clin Nutr. 2002 Jul;76(1):93-9.

32. Sthijns MM, Randall MJ, Bast A, Haenen GR. Adaptation to acrolein through upregulating the protection by glutathione in human bronchial epithelial cells: the materialization of the hormesis concept. Biochem Biophys Res Commun. $2014 \mathrm{Apr}$ 18;446(4):1029-34.

33. Sthijns MM, Thongkam W, Albrecht C, Hellack B, Bast A, Haenen GR, et al. Silver nanoparticles induce hormesis in A549 human epithelial cells. Toxicology in vitro : an international journal published in association with BIBRA. 2017 Apr;40:223-33.

34. Sthijns MM, Schiffers PM, Janssen GM, Lemmens KJ, Ides B, Vangrieken $P$, et al. Rutin protects against $\mathrm{H} 2 \mathrm{O} 2$-triggered impaired relaxation of placental arterioles and induces Nrf2-mediated adaptation in Human Umbilical Vein Endothelial Cells exposed to oxidative stress. Biochimica et biophysica acta. 2017 Mar 07;1861(5 Pt A):1177-89.

35. Mattson MP. Hormesis defined. Ageing Res Rev. 2008 Jan;7(1):1-7.

36. Wijnhoven SWP, Peijnenburg WJGM, Herberts CA, Hagens WI, Oomen AG, Heugens EHW, et al. Nano-silver - a review of available data and knowledge gaps in human and environmental risk assessment. Nanotoxicology. 2009;3:109-38.

37. Lilienblum W, Dekant W, Foth H, Gebel T, Hengstler JG, Kahl R, et al. Alternative methods to safety studies in experimental animals: role in the risk assessment of chemicals under the new European Chemicals Legislation (REACH). Archives of toxicology. 2008 Apr;82(4):211-36.

38. Bein K, Leikauf GD. Acrolein - a pulmonary hazard. Mol Nutr Food Res. 2011 Sep;55(9):1342-60.

39. Sung JH, Ji JH, Park JD, Yoon JU, Kim DS, Jeon KS, et al. Subchronic inhalation toxicity of silver nanoparticles. Toxicol Sci. 2009 Apr;108(2):452-61. 
40. Van De Wier B, Koek GH, Bast A, Haenen GR. The potential of flavonoids in the treatment of non-alcoholic fatty liver disease. Critical reviews in food science and nutrition. 2017 Mar 04;57(4):834-55.

41. Nilsson PM, Boutouyrie P, Laurent S. Vascular aging: A tale of EVA and ADAM in cardiovascular risk assessment and prevention. Hypertension. 2009 Jul;54(1):3-10.

42. Lemmens KJ, Vrolijk MF, Bouwman FG, van der Vijgh WJ, Bast A, Haenen GR. The minor structural difference between the antioxidants quercetin and 4'O-methylquercetin has a major impact on their selective thiol toxicity. Int J Mol Sci. 2014 Apr 30;15(5):747584.

43. Moalin M, van Strijdonck GP, Bast A, Haenen GR. Competition between ascorbate and glutathione for the oxidized form of methylated quercetin metabolites and analogues: tamarixetin, 4'O-methylquercetin, has the lowest thiol reactivity. Journal of agricultural and food chemistry. 2012 Sep 12;60(36):9292-7. 


Dankwoord 


\section{Dankwoord}

Dit zijn ze dan. De laatste pagina's van mijn boekje. Het einde is in zicht. Het was een leerzame, drukke periode met dit boekje als resultaat. Maar voordat $u$ aan het eind bent gekomen van deze thesis, wil ik graag nog de mensen bedanken die mij hebben geholpen, bijgestaan, gesteund, iets hebben geleerd of op een andere manier hebben bijgedragen tot de totstandkoming van dit boekje.

Allereerst wil ik mijn promotieteam bedanken!

Beste Aalt, heel erg bedankt voor je enthousiasme, onuitputtende motivatie, energie en positiviteit. Jouw deur staat altijd open. Bedankt voor de wekelijkse werkbesprekingen, waarin we samen met Guido de resultaten van die week bespraken. Ook als de resultaten onverwacht waren, wist jij mij altijd weer te motiveren en mijn nieuwsgierigheid te wekken om die week weer met een nieuwe vraagstelling en bijbehorende experimenten aan de slag te gaan.

Beste Guido, bedankt voor je kennis en inzichten. Ik heb veel van je geleerd en ik herinner me nog hoe je me verraste met een geheel nieuwe wijze van analyseren of hoe je me een geheel andere kijk gaf op resultaten, waardoor een artikel een geheel andere vernieuwende wending kon krijgen. Je bent heel erg betrokken bij het onderzoek.

Beste Antje, ik wil je graag bedanken. Ik heb niet alleen veel van je geleerd wat betreft het doen van wetenschappelijk onderzoek, maar ik kon ook altijd bij je terecht voor andere dingen. Ik herinner me de middagen dat we samen zaten te 'sparren' over hoe we bepaalde resultaten het beste konden weergeven in een manuscript. Ook heb je me heel erg geholpen als coach tijdens mijn BKO traject, waardoor ik ook mijn (onderwijs)competenties verder kon ontwikkelen. Ik bewonder je gedreven, kritische, beargumenteerde kijk en openheid tijdens onze vele brainstormsessies. En heb er heel veel zin in om nog eens samen met jou en je hondje Bruni te gaan wandelen!

Ook wil ik graag de leden van de beoordelingscommissie bedanken voor het beoordelen van mijn proefschrift.

Tijdens mijn PhD heb ik de mogelijkheid gehad om een deel van mijn onderzoek in samenwerking met verschillende onderzoeksgroepen en onderzoekers in het buitenland uit te voeren. Graag wil ik een aantal mensen bedanken.

Allereerst Roel, bedankt dat ik bij jouw groep op het IUF in Düsseldorf het project over nanoparticles mocht komen uitvoeren. Ook bedankt dat ik altijd vanuit Voerendaal 
met jou en Paul mocht meerijden naar Düsseldorf. Ik ben op de snelweg nog altijd op zoek naar de roofvogels. En in het donker, ga ik de vrachtwagens met lampjes tellen.

Waluree Thongkam, dear Waluree, it was really nice to perform all the experiments together. I think we learnt a lot from each other, regarding research but also as a person. Thank you for the collaboration and I think we became real friends. Thank you for showing me Düsseldorf and you are always welcome in Maastricht!

Furthermore, I am also very honored to thank all the people from the Karolinska Institutet, in Stockholm, Sweden. I would like to devote a special word of thanks to Katarina, Elias, Irina, Fernando, Anna and Bastiaan. Katarina, thank you for your critical scientific view! I still learn a lot from you! Thank you also as a friend and for making me feel very welcome in Stockholm. It has become my second home. Elias, thank you for sharing your knowledge with me and all your help with experiments and writing of manuscripts! It was nice to meet both of you at the conference in Sant Feliù de Guixols. We will keep in touch! And I hope to visit Stockholm some time in the future again! You are always very welcome in Maastricht. Also a lot of thanks to Irina, Fernando, Anna and Bastiaan, for also next to research making my time in Stockholm a very, very good one! I remember our nice trips to the city center, afternoons and evenings at the climbing center. Bastiaan, "Stockholm is sjoen, mer niks geit boven Mestreech. Bedaank dat ste mich un paar woorde Zweeds hubs gelierd! Tack så mycke!"

Dan wil ik natuurlijk graag mijn collega's bedanken. In het bijzonder, mijn medeAIO's Rianne F., Charlotte, Quan, Sven, Misha, Carmen, Philippe, Timme, Ming en Pauline V. Bedankt voor de gezellige tijd. Ik zal de vele etentjes, Pizza and Movie Nights, gezellige lunches, koffie-pauzes en andere activiteiten nooit vergeten! Vaak ook georganiseerd door (oud-collega) Roger B.! Bedankt allemaal! Ik zal jullie missen!

Aan mijn paranimf Misha: Mish, bedankt! Het was fijn altijd bij je binnen te kunnen lopen en even te kletsen. Bedankt voor je steun, vooral ook op het laatste. Ook heel veel succes met jouw promotie! Tegen de tijd dat ik mijn proefschrift ga verdedigen, heb jij het al achter de rug.

Ook wil ik graag de analisten bedanken. Beste Lou, Marie-José, Daniëlle P., Lily en Ger, heel erg bedankt voor jullie advies wat betreft technieken, bestellingen die jullie voor mij gedaan hebben, maar vooral ook bedankt dat ik bij jullie binnen mocht komen voor een gezellig praatje! Ook bedankt aan Eddy! De vrolijkheid waarmee jij iedere dag weer de pakketjes bezorgt, bezorgt iedereen een glimlach op zijn/haar gezicht. 
Marie-Claire en Josephina, heel erg bedankt voor jullie luisterend oor en voor alle hulp om de administratieve dingen voor mijn promotie te regelen.

Ook wil ik de andere collega's Frederik-Jan, Antoon, Harry, Matthijs, Ben, Roger G., Jan, Agnes, Gertjan, Geja, Paul, Alex, Agi, Sébastien, Agnieszka Br., Jacques, Helma en Peter bedanken voor de fijne werksfeer.

Daarnaast wil ik ook mijn oud-collega's bedanken: Roger B., Danielle B., Leonie, Joy, Alie, Kevin, Gesiele, Matt(hew), Erik, Edwin, Agnieszka Ba., Kristien en Bregje.

Ook een speciaal woord van dank aan mijn studenten: Bart, Sarah Sayed, Sarah Schmidt, Cassy en Puji. Bedankt voor jullie inzet en succes met jullie verdere studie en carrière!

Verder wil ik graag Pieter nog bedanken voor het beantwoorden van al mijn computervragen!

Bedankt Freek, van Humane Biologie, voor je hulp en ervaring met de massa spectrometrie experimenten.

Bedankt Geraldine dat ik iedere dag weer in een schoon kantoor aan de slag kon.

Tijdens mijn periode bij Farmacologie en Toxicologie heb ik mijn kantoor mogen delen met een aantal leuke kamergenootjes. Bedankt Daniëlle B., Bart, Pauline H., Kevin en Ming. Daniëlle, bedankt voor je gezelligheid en enthousiasme. Ik heb de kerstboom nadat je weg was nog ieder jaar opgezet en de snoepjes pot telkens opnieuw gevuld. Beide heb ik nu doorgegeven aan Ming. Bart, Pauline en Kevin bedankt voor jullie vrolijkheid en gezellige praatjes tijdens het werk. Ming, thank you for cooking a Chinese dinner for me and learning me a few words Chinese: Nĭ hăo Ming! And we always finished the day with: Mireille 'Wăn ān Ming!' Ming 'Fijne avond Mireille!'.

Ook wil ik graag mijn collega's van TGX bedanken, die mij onder andere vaak hebben vergezeld tijdens de congressen die ik bezocht heb.

Aan mijn nieuwe collega's bij MERLN, ik zie ernaar uit mijn Post-Doc bij jullie te starten.

Dan wil ik graag mijn vrienden bedanken. In het bijzonder mijn korfbalvrienden van de korfbalvereniging KV Maastricht, mijn muziekvrienden van Harmonie Heer Vooruit, met name de harmonieuze vriendinnen en de leden van badmintonclub BC Amby. Bedankt dat ik na een drukke werkdag, even lekker kon ontspannen! Maar vooral ook 
bedankt voor de gezelligheid en leuke activiteiten, etentjes, stapavonden en uitjes! Tot er nog heel veel mogen volgen!

Daarnaast wil ik graag mijn vrienden en vriendinnen van het "dansen" bedanken: Bedankt Nikkie, Alex, Guido, Nathan, Stephanie, Patrick, Maartje en Renske.

Renske, bedankt voor je hulp bij de stellingen!

Dankjewel aan mijn oudklasgenootjes en studiegenootjes Timo, Sabine, Roger en Rianne R.!

Rianne R., de spreuk "Rozen verwelken, scheepjes vergaan, maar onze vriendschap blijft altijd bestaan" is ons op het lijf geschreven. We kennen elkaar al sinds de basisschool. Je bent er altijd voor me. We kunnen uren kletsen aan de telefoon, gaan shoppen, op vakantie gaan, klimmen en wandelen. Hopelijk gaan we nog veel samen beleven! Bedankt!

Graag wil ik de hele familie bedanken en in het bijzonder mijn peter en meter opa Sthijns en oma Hermans. Van jullie heb ik geleerd niet op te geven en er in het leven vastberaden en $100 \%$ voor te gaan, iets wat ik tijdens mijn PhD goed heb kunnen gebruiken. Opa Dingo, 'iech blief altied Sthijns met $\mathrm{S} t \mathrm{~h}$ lange ij $\mathrm{n}$ s en zal blieve liere tot mien vingerkes even lang zeen. Oma Joepie, wij zijn twee handen op één buik en dat zal ook altijd zo zijn. Dankjulliewel voor alles wat ik van jullie heb geleerd en ik ben er trots op dat ik jullie kleindochter ben!

Ook bedankt aan opa Hermans, die helaas al overleden is, maar van $\mathrm{u}$ heb ik geleerd van het leven te genieten. Dank u wel oma Sthijns, u leerde mij de geheimen van de Limburgse keuken en gebak.

Natuurlijk wil ik ook de rest van mijn familie bedanken, namelijk tante Monique en 'noonk' Joost, mijn nichtjes Maud en Manon, tante Winny en 'noonk' Jos, mijn neef Bernd en nichtje Richelle en aanhang.

En nog een heel speciaal woord van dank aan tante Monique. U staat altijd voor mij klaar. Ook toen ik het moeilijk had, heeft u me erdoorheen gesleept en dat zal ik nooit vergeten. Zonder u zou ik dit nooit hebben bereikt. Dankjewel hiervoor!

Ook wil ik graag de schoonfamilie bedanken. Bedankt dat jullie mij zo hebben opgenomen. Jullie zitten diep in mijn hart. 
Chantalle, jij bent een heel bijzonder mens met een heel groot hart, altijd even behulpzaam en vrolijk. Met jou kan ik lachen en huilen. Je staat altijd open voor een praatje en steunt me door dik en dun. Je bent niet alleen mijn kleine zusje, maar ook gewoon een hele goede vriendin. Wij doen veel leuke dingen samen. Shoppen, sporten, muziek maken en dansen. Maar ook zeilen, rafting, klimmen en wandelen of de menige culturele stedentrips of vakanties. Ook in Zweden ben je me komen opzoeken. Bedankt voor al jouw steun! Ik wist al bij de start van mijn PhD dat ik jou als paranimf zou kiezen en dat is tijdens het project alleen maar bevestigd. Via deze weg wil ik je bedanken voor alles! Ik ben er trots op dat ik jouw grote zus mag zijn. Van jou kunnen nog veel mensen wat leren.

Lieve Papa, dankjewel voor je geduld en steun! Je bent voor mij echt een voorbeeld. Ik bewonder jouw enorme hoeveelheid aan kennis en ervaring op verschillende vakgebieden. Bedankt dat ik altijd met een vraag bij jou terecht kan en dat je altijd voor mij klaarstaat.

Lieve Mama, ik heb zelden zo een sterk persoon zoals jou gezien. Onvoorwaardelijk sta jij altijd voor iedereen klaar! Je hebt me altijd geholpen en gesteund, ook als het moeilijk was.

Lieve Papa en Mama, ontzettend bedankt, zonder jullie was dit boekje er niet geweest! Alles wat ik tot nu toe in mijn leven heb bereikt, heb ik niet alleen aan mezelf, maar vooral aan jullie te danken. Jullie hebben me de kans gegeven te doen en bereiken wat ik wilde en me ook in iedere keuze die ik maakte onvoorwaardelijk gesteund. Jullie zijn mijn 'Lichtlein'. Bedankt voor jullie liefde! Ik hoop nog veel samen met jullie van het leven te genieten!

Jarno, miene sjat, heel erg bedankt voor al jouw steun. Ik houd ontzettend veel van jou, de liefde van mijn leven! Ik heb je leren kennen op de afdeling, jij werkte bij TGX en ik bij TOX. Je hebt me altijd gesteund, ook tijdens de moeilijke momenten. Je wist me altijd weer op te vrolijken. Ik bewonder je rust, positiviteit en relativeringsvermogen! Alles kump good! En ik zie ernaar uit om samen te gaan wonen en de rest van mijn leven samen met jou te delen. 




\section{List of publications}

1. Sthijns MMJPE, Weseler AR, Schmidt S, Arnér ESJ, Johansson K, Bast A, Haenen GRMM. Redox modulation determines the fate of human melanoma cells exposed to quercetin and oxidative stress: adaptive antioxidant protection or apoptosis, a matter of life or death. In progress.

2. Sthijns MMJPE, Den Hartog GJM, Scasso C, Haenen JP, Bast A, Haenen GRMM. Dual effect of acrolein on microsomal glutathione S-transferase 1 activity: Effect of concentration and time. Submitted.

3. Sthijns MMJPE, Schiffers PM, Janssen GM, Lemmens KJA, Ides $B$, Vangrieken $P$, Bouwman FG, Mariman EC, Pader I, Arnér ESJ, Johansson K, Bast A, Haenen GRMM. Rutin protects against hydrogen peroxide-triggered impaired relaxation of placental arterioles and induces Nrf2-mediated adaptation in Human Umbilical Vein Endothelial Cells exposed to oxidative stress. Biochimica Biophysica Acta General Subjects. 2017 Mar 7;1861(5 Pt A):1177-1189. Published.

4. Sthijns MMJPE, Thongkam W, Albrecht C, Hellack B, Bast A, Haenen GRMM, Schins RPF. Hormesis of silver nanoparticles in A549 human epithelial cells. Toxicology in vitro 2017 Jan 18;40:223-233. Published.

5. Sthijns MMJPE, Weseler AR, Bast A, Haenen GRMM. Time in redox adaptation processes: from evolution to hormesis. International Journal of Molecular Sciences 2016 Sep 29;17(10). Published.

6. Lemmens KJ, Sthijns MMJPE, van der Vijgh WJF, Bast A, Haenen GRMM. The antioxidant flavonoid monoHER provides efficient protection and induces the innate Nrf2 mediated adaptation in endothelial cells subjected to oxidative stress. PharmaNutrition 2014 July;2(3):69-74. Published.

7. Sthijns MMJPE, Randall MJ, Bast A, Haenen GRMM. Adaptation to acrolein through upregulation the protection by glutathione in human bronchial epithelial cells: The materialization of the hormesis concept. Biochemical and Biophysical Research Communications 2014 Apr 18;446(4):1029-34. Published. 



\section{List of abstracts}

1. Sthijns MMJPE, Schiffers PM, Bouwman FG, Mariman ECM, Arnér ESJ, Johansson $\mathrm{K}$, Bast $\mathrm{A}$, Haenen GRMM. Rutin protects against $\mathrm{H}_{2} \mathrm{O}_{2}$ triggered impaired relaxation of placental arterioles and induces Nrf2-mediated adaptation in HUVECs exposed to oxidative stress. Society Of Toxicology, annual meeting, poster presentation, Baltimore, United States, abstract published in The Toxicologist.

2. Sthijns MMJPE, Thongkam W, Albrecht C, Hellack B, Bast A, Haenen GRMM, Schins RPF. Silver nanoparticles induce adaptation in A549 human epithelial cells. Annual NUTRIM Symposium 2016, poster presentation, Maastricht University.

3. Sthijns MMJPE, Schiffers PM, Janssen GM, Ides B, Vangrieken P, Bouwman FG, Mariman EC, Pader I, Arnér ESJ, Johansson K, Bast A, Haenen GRMM. Rutin protects against endothelial dysfunction and induces Nrf2-mediated adaptation in HUVECs exposed to oxidative stress. Netherlands Vascular Biology Meeting 2016, Biezenmortel, The Netherlands, poster presentation and invited speaker.

4. Sthijns MMJPE, Bouwman FG, Johansson K, Schiffers PM, Bast A, Haenen GRMM. Rutin protects against endothelial dysfunction and induces Nrf2-mediated adaptation in HUVECs exposed to oxidative stress. Eurotox 2016, European Conference of European Societies of Toxicology, poster presentation, Sevilla, Spain, abstract published in Toxicology Letters.

5. Sthijns MMJPE, Bouwman FG, Johansson K, Schiffers PM, Bast A, Haenen GRMM. Rutin protects against endothelial dysfunction and induces Nrf2-mediated adaptation in HUVECs exposed to oxidative stress. Dutch society of toxicology conference 2016, poster presentation, invited speaker and pitch, Soesterberg, the Netherlands. PhD poster presentation prize.

6. Sthijns MMJPE, Johansson K, Arnér ESJ, Bouwman FG, Ides B, Bast A, Haenen GRMM. Effects of the flavonoid rutin on mammalian redox systems. Annual NUTRIM Symposium 2015, poster presentation, Maastricht University.

7. Sthijns MMJPE, Bast A, Arnér ESJ, Johansson K, Haenen GRMM. The effects of flavonoids on redox regulation. Conference 'Thiol-based Redox Switches in Life 
Sciences' 2015, poster presentation and pitch, Sant Feliù de Guixols, Spain. Poster presentation prize.

8. Sthijns MMJPE, Randall MJ, Bast A, Haenen GRMM. The materialization of the hormesis concept: Adaptation to acrolein in human bronchial epithelial cells. Annual NUTRIM Symposium 2014, poster presentation, Maastricht University.

9. Sthijns MMJPE, Randall MJ, Bast A, Haenen GRMM. The materialization of the hormesis concept: Adaptation to acrolein in human bronchial epithelial cells. MOSA Conference 2014, poster presentation, Maastricht University. 




\section{Curriculum vitae}

Mireille Maria Johanna Petronella Elisabeth Sthijns was born on February $25^{\text {th }}, 1991$ in Maastricht, the Netherlands. From 2003 to 2009, she attended secondary school at 'Porta Mosana College' in Maastricht, which she finished with distinction cum laude. In September 2009 she started the Bachelor Biomedical Sciences, specialization Molecular Life Sciences. After the Bachelor she continued with the Master Biomedical Sciences. In June 2014, she graduated specialized in Nutrition and Metabolism. She finished her Masters programme with an internship in which she investigated the activity of flavonoids and acrolein for which she won the SWUM (Stichting Wetenschapsbeoefening Universiteit Maastricht) price 2015 for best master thesis of 2014. Both her Bachelor and Master she finished with distinction cum laude. Due to the achievement of the Kootstra Talent Fellowship she was able to start her PhD at the Department of Pharmacology and Toxicology on the project: "Protection, adaptation or cell death as response to toxins: Dependence on time and dose". During this period she started a collaboration with Prof. Dr. Roel P.F. Schins from the IUF Institute - Leibniz Research Institute for Environmental Medicine, in Düsseldorf, Germany. At the IUF institute she got experience to work with nanoparticles and investigated the adaptation phenomenon for these nanoparticles in detail. Because of the presence of a model cell system to examine the effect of antioxidants on different redox signaling pathways, she decided to write for the Jo Kolk Studiefonds, which she received in December 2014 and that enabled her to finance her stay at the lab of Prof. Dr. Elias S.J. Arnér and Dr. Katarina Johansson at the Karolinska Institutet in Stockholm, Sweden. This research was successfully completed and resulted in interesting data for which she received twice a prize for the best poster. The first prize was awarded in September 2015 at a conference organized by the European Science Foundation (ESF) in partnership with European Molecular Biology Organisation (EMBO) about 'Thiol-based Redox Switches in Life Sciences' in Sant Feliù de Guixols, Spain and the second in June 2016 at the Dutch society of toxicology meeting in Soesterberg, The Netherlands. The research performed during this project and described in this thesis was under the supervision of Prof. Dr. Aalt Bast, Prof. Dr. Guido R.M.M. Haenen and Dr. Antje R. Weseler. 


